\title{
A Search for Neutral Supersymmetric Higgs Bosons at DØ
}

\author{
Nicolas Ahmed Osman \\ Imperial College London
}

A thesis submitted for the degree of

Doctor of Philosophy

of the University of London

and the Diploma of Imperial College

September, 2010 


\title{
A Search for Neutral Supersymmetric Higgs Bosons at DØ
}

\author{
Nicolas Ahmed Osman \\ Imperial College London
}

September, 2010

\begin{abstract}
A search for Higgs bosons in multijet data from the $\mathrm{D} \varnothing$ detector is reported in this thesis. The Higgs boson is the only remaining undiscovered particle in the Standard Model of particle physics, and plays an integral role in this model. It is known that this model is not a complete description of fundamental physics (it does not describe gravity, for example), and so searches for physics beyond the Standard Model are an important part of particle physics. One extension of the Standard Model, the Minimal Supersymmetric Standard Model (MSSM), predicts the existence of five Higgs bosons, two of which can show an enhanced coupling to bottom quarks. For this reason, a search in the $b b b$ (multijet) channel is a sensitive test of Higgs boson physics.

The analysis described in this thesis was conducted over $6.6 \mathrm{fb}^{-1}$ of data. At the time of writing, the best limits on $\tan \beta$ (a key parameter of the MSSM) in the multijet channel were set by $\mathrm{D} \varnothing[1]$. The new analysis described in this thesis included more data than the previous analysis in the channel, and made use of a new trigger and event-based analysis method. An improved Multivariate Analysis technique was used to separate signal and background events and produce a final discriminant for the limit setting process. These changes increased the expected sensitivity of this measurement by roughly $50 \%$ more than would be expected from the increase in the size of data sample alone.
\end{abstract}




\section{Acknowledgements}

I would like to thank my supervisors, Jon Hays, Gavin Davies and Rick Jesik, for their guidance and support for these past few years. I would also like to thank the other members of the Imperial College DØ Group (Per Jonsson and Tim Scanlon) for all their help and advice, as well as their work on the multijet search, of which the analysis in this thesis is an extension.

Many thanks to the Higgs conveners and Physics conveners at DØ for their advice on how to proceed with the analysis. I think it is also appropriate to thank the b-Jet Identification group and their conveners: the b-tagging algorithms at DØ are exceptional, and are the driving factor behind many of the high quality results $\mathrm{D} \varnothing$ is currently producing.

I would like to thank STFC for providing the funding for this research.

I thank my family for all their support and the sacrifices they have made for me. Finally, many thanks to Megumi, primarily for her patience and encouragement, and of course for her concerts, recitals and recordings which have greatly helped me throughout the $\mathrm{PhD}$. 


\section{Table of Contents}

$\begin{array}{ll}\text { Abstract } & 2\end{array}$

Acknowledgements $\quad 3$

Table of Contents $\quad 4$

$\begin{array}{ll}\text { List of Figures } & 7\end{array}$

$\begin{array}{ll}\text { List of Tables } & 17\end{array}$

$\begin{array}{ll}\text { Introduction } & 19\end{array}$

Chapter 1. Theory 21

1.1 Local Gauge Theories 21

1.2 The Higgs Mechanism 24

1.2.1 The Higgs Mechanism in the Standard Model 25

1.3 Constraints on the Standard Model Higgs Boson 26

1.3.1 Theoretical Constraints 27

1.3.2 Direct Searches 27

1.3.3 Indirect Measurements 28

1.4 Beyond the Standard Model 30

1.4.1 Supersymmetry and the MSSM Higgs Sector 30

1.4.2 Constraints on the Neutral MSSM Higgs Bosons 34 
Chapter 2. The DØ Experiment $\quad 35$

2.1 The Tevatron $\quad 35$

2.2 The DØ Detector $\quad 35$

$\begin{array}{lll}\text { 2.2.1 Tracking Detector } & 37\end{array}$

$\begin{array}{ll}2.2 .2 \text { Solenoid } & 40\end{array}$

2.2.3 Preshower Detectors 41

2.2.4 Calorimeter 41

2.2.5 Muon Detector 43

2.2.6 Luminosity Monitor 44

$\begin{array}{lll}2.2 .7 & \text { Trigger } & 44\end{array}$

2.2.8 Offline Reconstruction Software 46

2.2 .9 b-Jet Identification $\quad 47$

2.2.10 Detector Performance 50

Chapter 3. Search for Neutral Supersymmetric Higgs Bosons in the Multijet Channel

$\begin{array}{lll}3.1 & \text { Introduction } & 52\end{array}$

3.1.1 $\phi \mathrm{b}(\mathrm{b}) \rightarrow b \bar{b} b(b)$ in the MSSM

3.1.2 Overview of Previous Analysis Versions 54

3.2 Analysis Method $\quad 55$

3.2.1 Data Sample $\quad 56$

$\begin{array}{ll}\text { 3.2.2 } & \text { Monte Carlo Samples } \\ & 57\end{array}$

$\begin{array}{lll}3.2 .3 & \text { b-Tagging } & 58\end{array}$

3.2.4 Event Selection $\quad 59$

3.2.5 Background Composition 61

3.2.6 Background Prediction 66

$\begin{array}{lll}3.3 & \text { Conclusion } & 69\end{array}$

Chapter 4. Overview of Multivariate Methods for the Multijet Anal$\begin{array}{ll}\text { ysis } & 70\end{array}$

$\begin{array}{lll}4.1 & \text { Introduction } & 70\end{array}$

$\begin{array}{lll}4.2 & \text { Neural Networks } & 71\end{array}$ 
4.3 Forest of Decision Trees with Boosting (BDT) Method 73

4.4 Forest of Randomised Decision Trees (RF) Method 74

4.5 Random Forest Input Variables and Outputs 76

4.6 Conclusion $\quad 89$

Chapter 5. Confidence Limits on $\sigma \times B R$ for the Process $\phi b \rightarrow b b b \quad 90$

$\begin{array}{lll}5.1 & \text { Introduction } & 90\end{array}$

5.2 Systematic Uncertainties 90

5.2.1 Systematic Uncertainties on the Signal 91

5.2.2 Systematic Uncertainties on the Background 94

$\begin{array}{lll}5.3 & \text { Results } & 104\end{array}$

5.3.1 Model Independent and Naive MSSM Confidence Limits 109

5.3.2 Confidence Limits for Benchmark MSSM Scenarios 114

5.4 Conclusion 119

$\begin{array}{lll}\text { Chapter 6. Conclusion and Outlook } & 120\end{array}$

$\begin{array}{llr}6.1 \text { Conclusion } & 120\end{array}$

6.2 Outlook 121

$\begin{array}{ll}\text { References } & 123\end{array}$ 


\section{List of Figures}

1.1 The potential $V$, defined in Equation (1.13), as a function of $\Phi$, for the case (a) $\mu^{2}>0$ and (b) $\mu^{2}<0$. In case (a), $\mu$ is a physical mass and the vacuum expectation value of $\Phi$ is 0 , while in case (b) there is a continuous range of VEVs.

1.2 Theoretical constraints on the Higgs boson mass as a function of energy scale $\Lambda$ [15]. The parameter space above (below) the upper (lower) grey band is excluded.

1.3 Combined confidence limits on SM Higgs production from the Tevatron experiments [24]. The plot shows the value of $\sigma \times B R$ excluded to $95 \%$ confidence divided by the theoretical value as a function of Higgs boson mass. Where the solid black (observed limit) line is below one, the SM Higgs boson is excluded to $95 \%$ confidence.

$1.4 \Delta \chi^{2}$ for fits of EW parameters for different Higgs masses, where $\Delta \chi^{2}=$ $\chi^{2}\left(m_{H}\right)-\chi_{\min }^{2}[25]$. The best fit occurs at $85 \mathrm{GeV}$.

1.5 The inverse of $\alpha_{1}, \alpha_{2}$ and $\alpha_{3}$ (proportional to the square of the $\mathrm{U}(1)$, $\mathrm{SU}(2)$ and SU(3) coupling constants respectively) for the SM (left) and MSSM (right), as a function of energy scale Q in GeV [26].

1.6 The tree level masses of the $H$ (top) and $h$ (bottom) bosons as a function of $m_{A}$ for different $\tan \beta$ values, given by Equation (1.24). As $\tan \beta$ increases, a mass degeneracy where $m_{h} \approx m_{A}$ or $m_{H} \approx m_{A}$ occurs. When radiative corrections are applied, the maximum value of $m_{h}$ increases from $m_{Z}$ to roughly $130 \mathrm{GeV}$ 
2.1 The Tevatron and accelerator chain. Hydrogen atoms are ionised using an electric field generated using a Cockroft-Walton voltage multiplier. The resulting protons line the caesium cathode, and can combine with electrons from the caesium to form $\mathrm{H}^{-}$ions (caesium is used because of its low work function). These are repelled by the cathode and directed through a graphite target to strip away the electrons. The resulting protons are transferred to the booster, which raises their energy to 8 $\mathrm{GeV}$, and then to the Main Injector. Some protons are directed to a nickel target to produce antiprotons. The protons and antiprotons are accelerated to energies of $150 \mathrm{GeV}$ before being injected into the Tevatron (aerial photograph from [40]).

2.2 The full D $\varnothing$ detector, including (from centre outwards) tracking, preshower, calorimeter and muon sub-detectors. On the diagram the $\mathrm{z}$ axis runs from left to right through the centre of the detector, and the y-axis runs from bottom to top [41].

2.3 The $\mathrm{D} \varnothing$ tracking system within the solenoid. The barrels and disks of the silicon microstrip tracker are visible towards the centre, with the layers of fibre tracker arranged around them. The preshower detectors are also seen on the inside surface of the calorimeter [41].

2.4 The SMT detector. The silicon microstrips are arranged in a number of barrels and disks to enable tracking in the region $|\eta|<3[41]$.

2.5 Cross section of the $\mathrm{D} \varnothing$ calorimeter, displaying the electromagnetic, fine hadronic and coarse hadronic layers [41].

2.6 Detail of the calorimeter cells, also showing the inter-cryostat region and pre-shower detector [41].

2.7 An example b decay. The decay length is marked as $L_{x y}$ and the IP is denoted $d_{0}[45]$.

2.8 The trigger efficiency with respect to offline reconstruction, plotted as a function of trigger rate for the SVT trigger. The points represent decay length significance cuts of 8, 6, 3, 2, 1 and 0 from left to right. [57] 
2.9 The D $\varnothing$ daily data taking efficiency for the period October 2009 to September 2010. The data taking efficiency is the ratio of recorded to delivered data. The efficiency is generally above 90\%, allowing a large amount of data to be collected and analysed [59].

3.1 Higgs boson branching ratios as a function of Higgs mass for the (a) the Standard Model and (b) the MSSM [61]. The MSSM graph gives branching ratios for the $A$ boson; the $A$ boson coupling to up type quarks is suppressed, and the $A$ boson does not couple to the weak bosons.

3.2 Feynman diagrams for (a) three and (b) four jet $\phi b(b) \rightarrow b \bar{b} b(b)$ decays. The process $b \rightarrow \phi b$ is enhanced by a factor of $\tan ^{2} \beta$ in the MSSM.

3.3 Median expected (dotted blue line) and observed (solid red line) limits on $\sigma \times B R$ for the $\phi b \rightarrow b \bar{b} b$ process as a function of Higgs boson mass, using $1 \mathrm{fb}^{-1}$ of DØ RunII data [66]. The green and yellow bands correspond to the expected limit where the expected number of events is varied within $\pm 1 \sigma$ and $\pm 2 \sigma$ respectively.

3.4 Composition of zero to three $b$-tagged three jet data samples for RunIIb. Numerical values for each of the $b$-tagged samples are shown in Table 3.8. This method was also applied to four-jet data and RunIIa data.

3.5 Composition of zero to three $b$-tagged three jet data samples for RunIIb plotted on a logarithmic scale. Numerical values for each of the $b$-tagged samples are shown in Table 3.8. This method was also applied to four-jet data and RunIIa data. The total MC background $H_{T}$ distribution agrees well with that for the data: this was achieved by fitting the MC to data for each individual bin in the $H_{T}$ distribution.

3.6 The transverse momentum $\left(p_{T}\right)$ distributions for the leading (top left), second (top right) and third (bottom left) jets in $p_{T}$ order for p20 data in the 3 -jet channel.

3.7 The pseudorapidity $(\eta)$ distributions for the leading (top left), second (top right) and third (bottom left) jets in $p_{T}$ order for p20 data in the 3-jet channel. 
3.8 The transverse momentum $\left(p_{T}\right)$ distributions for the leading (top left), second (top right) and third (bottom left) jets in $p_{T}$ order for p20 data in the 4-jet channel.

3.9 The pseudorapidity $(\eta)$ distributions for the leading (top left), second (top right) and third (bottom left) jets in $p_{T}$ order for p20 data in the 4 -jet channel.

4.1 Diagram illustrating the structure of a simple neural network. The illustrated NN consists of three layers of nodes (illustrated as blue circles): an input layer (left), a hidden layer (centre) and an output layer (right). The edges which link the nodes are also shown.

4.2 Diagram illustrating the structure of a simple decision tree (DT). The green circles represent samples that do not meet the purity criterion, while the red and blue circles are samples below the purity threshold, which are dominated by signal and background events respectively. At the first node (at the top of the diagram), the DT tests a number of possible cuts on each variable, and uses the cut which provides the best signal-background separation. This process is repeated until all samples reach the purity criterion, or the maximum depth of the tree is reached.

4.3 Output values of signal (red) and background (blue) MC samples for BDT method after (a) 20, (b) 50 and (c) 100 trees. The signal and background distributions converge as the number of trees increases.

4.4 Random Forest output distributions for the signal (red) and background (blue) MC training samples. The signal and background RF output distributions peak at \pm 1 , indicating good discrimination between signal and background separation.

4.5 A comparison of expected limits on $\tan \beta$ using different MVA methods. The expected limits for the NN, BDT and RF methods are represented by the black, red and green lines respectively. For this comparison,all limits were set without systematic errors. The RF method was chosen because of its stability. The limit setting process is described in Chapter 5. 77 
4.6 The deviation angle illustrated for two Higgs candidates: the leading and second-leading jet, and the leading and third-leading jet. The candidates have masses $180 \mathrm{GeV}$ and $150 \mathrm{GeV}$ and are plotted on the $\mathrm{x}$ - and y-axis respectively. Events where the two quantities are equal would lie on the dotted line. The angle between the dotted and solid black lines is a measure of deviation of both masses from the mean. A three-dimensional version of this angle was used in this analysis.

4.7 The di-jet invariant mass distributions for jet pairs 12 (top left), 23 (top right) and 13 (bottom left) for p20 MC in the 3-jet channel. The blue histogram represents MC background, while the purple, red and orange histograms are signal distributions for $m_{h}$ of 120,180 , and $240 \mathrm{GeV}$ respectively.

4.8 The $\Delta \eta$ distributions for jet pairs 12 (top left), 23 (top right) and 13 (bottom left) for p20 MC in the 3-jet channel. The blue histogram represents MC background, while the purple, red and orange histograms are signal distributions for $m_{h}$ of 120, 180, and $240 \mathrm{GeV}$ respectively.

4.9 The opening angle distributions for jet pairs 12 (top left), 23 (top right) and 13 (bottom left) for p20 MC in the 3-jet channel. This angle is described in the list of RF input variables. The blue histogram represents MC background, while the purple, red and orange histograms are signal distributions for $m_{h}$ of 120,180 , and $240 \mathrm{GeV}$ respectively.

4.10 The event sphericity distribution for p20 MC in the 3-jet channel. The blue histogram represents MC background, while the purple, red and orange histograms are signal distributions for $m_{h}$ of 120, 180, and 240 GeV respectively.

4.11 The mass deviation angle distribution for p20 MC in the 3-jet channel. This variable is defined in the list of RF input variables. The blue histogram represents MC background, while the purple, red and orange histograms are signal distributions for $m_{h}$ of 120,180 , and $240 \mathrm{GeV}$ respectively.

4.12 The di-jet invariant mass distributions for jet pairs 12 (top left), 23 (top right) and 13 (bottom left) for RunIIb data and MC in the 3-jet channel. The signal histogram is for a Higgs boson with mass $180 \mathrm{GeV}$. 
4.13 The $\Delta \eta$ distributions for jet pairs 12 (top left), 23 (top right) and 13 (bottom left) for RunIIb data and MC in the 3-jet channel. The signal histogram is for a Higgs boson with mass $180 \mathrm{GeV}$.

4.14 The opening angle distributions for jet pairs 12 (top left), 23 (top right) and 13 (bottom left) for RunIIb data and MC in the 3-jet channel. This angle is described in the list of RF input variables. The signal histogram is for a Higgs boson with mass $180 \mathrm{GeV}$.

4.15 The event sphericity distribution for RunIIb data and MC in the 3-jet channel. The signal histogram is for a Higgs boson with mass $120 \mathrm{GeV}$.

4.16 The mass deviation angle distribution for RunIIb data and MC in the 3-jet channel. This variable is defined in the list of RF input variables. The signal histogram is for a Higgs boson with mass $120 \mathrm{GeV}$.

4.17 The random forest output distribution for a Higgs boson mass of 180 $\mathrm{GeV}$ in the 3-jet channel (RunIIa).

4.18 The random forest output distribution for a Higgs boson mass of 180 $\mathrm{GeV}$ in the 4-jet channel (RunIIa).

4.19 The random forest output distribution for a Higgs boson mass of 180 $\mathrm{GeV}$ in the 3-jet channel (RunIIb).

4.20 The random forest output distribution for a Higgs boson mass of 180 $\mathrm{GeV}$ in the 4-jet channel (RunIIb).

5.1 Systematic uncertainties on the signal due to the jet energy scale for the three jet channel in RunIIb data, plotted as a fraction of the nominal signal distribution (for a Higgs boson mass of $180 \mathrm{GeV}$ ). A second order polynomial was fitted to the positive and negative fluctuations.

5.2 Systematic uncertainties on the signal due to the JSSR for the three jet channel in RunIIb data, plotted as a fraction of the nominal signal distribution (for a Higgs boson mass of $180 \mathrm{GeV}$ ). A second order polynomial was fitted to the positive and negative fluctuations.

5.3 Systematic uncertainties on the signal, plotted as a fraction of the signal distribution as a function of the p17 three jet channel RF output for a Higgs boson mass of $180 \mathrm{GeV}$. 
5.4 Systematic uncertainties on the signal, plotted as a fraction of the signal distribution as a function of the p17 four jet channel RF output for a Higgs boson mass of $180 \mathrm{GeV}$.

5.5 Systematic uncertainties on the signal, plotted as a fraction of the signal distribution as a function of the p20 three jet channel RF output for a Higgs boson mass of $180 \mathrm{GeV}$.

5.6 Systematic uncertainties on the signal, plotted as a fraction of the signal distribution as a function of the p20 four jet channel RF output for a Higgs boson mass of $180 \mathrm{GeV}$.

5.7 Systematic uncertainties as a fraction of the nominal predicted background for positive fluctuations of (a) JES and (b) JSSR. When fitting a polynomial to these systematics, it was found that they were consistent with a flat systematic at 0 .

5.8 Systematic uncertainties as a fraction of the nominal predicted background plotted as a function of the p17 three jet channel RF output for a Higgs mass of $180 \mathrm{GeV}$. The systematics are shown both (a) unnormalised and (b) normalised to equal area with the predicted background. The black dotted line in (b) is intended to give an indication of the total uncertainty: this is not the true total because the scale of the nominal background is allowed to float in the limit setting process.

5.9 Systematic uncertainties as a fraction of the nominal predicted background plotted as a function of the p17 four jet channel RF output for a Higgs boson mass of $180 \mathrm{GeV}$. The systematics are shown both (a) un-normalised and (b) normalised to equal area with the predicted background. The black dotted line in (b) is intended to give an indication of the total uncertainty: this is not the true total because the scale of the nominal background is allowed to float in the limit setting process. 
5.10 Systematic uncertainties as a fraction of the nominal predicted background plotted as a function of the p20 three jet channel RF output for a Higgs mass of $180 \mathrm{GeV}$. The systematics are shown both (a) unnormalised and (b) normalised to equal area with the predicted background. The black dotted line in (b) is intended to give an indication of the total uncertainty: this is not the true total because the scale of the nominal background is allowed to float in the limit setting process.

5.11 Systematic uncertainties as a fraction of the nominal predicted background plotted as a function of the p20 four jet channel RF output for a Higgs boson mass of $180 \mathrm{GeV}$. The systematics are shown both (a) un-normalised and (b) normalised to equal area with the predicted background. The black dotted line in (b) is intended to give an indication of the total uncertainty: this is not the true total because the scale of the nominal background is allowed to float in the limit setting process.

5.12 The impact of the value of $R_{i}(5.4)$ for (a) the $t \bar{t}$ background contribution and (b) the heavy jet TRF systematic uncertainties on the overall LLRs, $Q_{n}$ for signal-plus-background (red) and background-only (black) cases. In these plots, the values of all other nuisance parameters apart from the one under investigation are fixed. The signal hypothesis in both plots is for a Higgs boson with $m_{A}=160 \mathrm{GeV}$ and $\tan \beta=50$. The top quark contribution was a relatively small uncertainty, and varying this parameter did not have a large effect on $Q_{n}$. The heavy-jet TRF, on the other hand, was a dominant source of uncertainty, and the LLR was more sensitive to this parameter. The minima of the signal-plus-background and background only $Q_{n}$ distributions occur at different values of $R_{i}$ : this shows that this uncertainty has a different effect for the two hypotheses. 105 
5.13 The values of $R_{i}$ (5.4) for (a) the top quark contribution and (b) the the heavy jet TRF uncertainties in each pseudo-experiment: these values optimise the overall likelihood. Note that, unlike in Figure 5.12, all parameters were allowed to fluctuate when minimising the likelihood. The width of the $R_{i}$ distributions in (a) is roughly 1 . The mean of the distributions in (a) is between 0.5 and 0.8 ; this indicates that the likelihood was optimised for a central value slightly higher than the nominal estimate. In the heavy jet TRF uncertainty plots, the widths of the distributions are less than one: this indicates that the fit constrains the value of this uncertainty. The signal and signal-plus-background distributions peak at different values of $R_{i}$, as they did in Figure 5.12 (b).

5.14 The LLR for the expected number of background-only (black) and signalplus-background (red) events plotted against $m_{A}$. The deviation of the two distributions indicates the degree of separation between the signal and background hypotheses. The yellow and green bands show the value of the LLR for the background-only hypothesis where the predicted number of background events has been increased and decreased by one and two standard deviations respectively: these allow the mean value of the signal-plus-background LLR distribution to be compared with the width of the background-only LLR distribution. The signal has been scaled according to Equation (3.1) with $\tan \beta=50$.

5.15 Expected confidence limits plotted versus $m_{A}$ : (a) the values of $\sigma \times B R$ for the $\phi b \rightarrow b \bar{b} b$ process and (b) $\tan \beta$ excluded to $95 \%$ confidence, as a function of neutral Higgs boson mass $\left(m_{A}\right)$. The $\tan \beta$ limit is calculated using Equation (3.1). The yellow and green bands represent the $\pm 1 \sigma$ and $\pm 2 \sigma$ variations around the expectation.

5.16 Comparison of expected confidence limits on $\sigma \times \mathrm{BR}$ for $5.2 \mathrm{fb}^{-1}$ (green) and $6.6 \mathrm{fb}^{-1}$ (black), plotted on (a) a linear and (b) a logarithmic scale. The increase in sensitivity is greater than that which would be expected for the increase in integrated luminosity analysed.

5.17 Comparison of expected confidence limits on $\sigma \times$ BR for $6.6 \mathrm{fb}^{-1}$ (black) and the same with doubled systematics. The decrease in sensitivity is roughly $20 \%$. 
5.18 The effective $\tan \beta$ plotted against nominal $\tan \beta$ for the MSSM scenarios considered in this thesis. The correction to $\tan \beta$ is given in (5.8). The black dotted line shows the uncorrected value of $\tan \beta$ (that is, the case where $\Delta_{b}=0$ for all $\left.\tan \beta\right)$.

5.19 Signal RF distributions for $m_{A}=180 \mathrm{GeV}$, $\tan \beta=60$, for different MSSM scenarios relative to the naive enhancement. The corrections to $\tan \beta$ affect the scale of the distribution. The increased Higgs boson width causes more signal events to be placed in the most background-like bins of the RF distribution.

5.20 Signal di-jet invariant mass distribution for the leading jet pair, for a Higgs boson mass of $180 \mathrm{GeV}$, with a $\tan \beta$ of 60 in the $m_{h}^{\max }, \mu=$ $-200 \mathrm{GeV}$ scenario. The predicted Higgs boson width for this scenario is approximately $25 \mathrm{GeV}$ : the width observed in is greater due to detector resolution effects. The contributions from the different mass hypotheses to the overall distribution are shown separately. The mass hypotheses closer to the test mass $(180 \mathrm{GeV})$ give the largest contribution, as expected.116

5.21 Expected confidence limits on $\tan \beta$ as a function of $m_{A}$, assuming the MSSM no mixing scenario $(\mu=-200 \mathrm{GeV})$. The yellow and green bands represent the $\pm 1 \sigma$ and $\pm 2 \sigma$ variations around the expectation. The region excluded by the LEP experiments is also shown [33].

5.22 Expected confidence limits on $\tan \beta$ as a function of $m_{A}$, assuming the MSSM no mixing scenario $(\mu=+200 \mathrm{GeV})$. The yellow and green bands represent the $\pm 1 \sigma$ and $\pm 2 \sigma$ variations around the expectation. The region excluded by the LEP experiments is also shown [33].

5.23 Expected confidence limits on $\tan \beta$ as a function of $m_{A}$, assuming the MSSM $m_{h}^{\max }(\mu=-200 \mathrm{GeV})$ scenario. The yellow and green bands represent the $\pm 1 \sigma$ and $\pm 2 \sigma$ variations around the expectation. The region excluded by the LEP experiments is also shown [33]. 


\section{List of Tables}

1.1 The MSSM enhancements over the SM for various neutral Higgs bosonquark interactions. $\alpha$ is a mixing angle, describing interactions between the two Higgs doublets [31].

2.1 A summary of Tevatron properties in Run I and Run II [40].

2.2 Event rate and latency for each trigger level at $\mathrm{D} \varnothing$

2.3 The fake rates and efficiencies for various operating points of the NN and MVA b-taggers. The range of outputs for both b-taggers is from 0 (less b-jet like) to 1 (more b-jet like).

3.1 List of triggers used in the p17 (RunIIa) analysis. $C J T(x, y,|\eta|<z)$ corresponds to a requirement of $x$ calorimeter trigger towers with energy greater than $y \mathrm{GeV}$ within $|\eta|<z$. The $J T(x, y,|\eta|<z)$ term indicates a requirement of $x$ jets with $p_{T}>y \mathrm{GeV}$ and $|\eta|<z$. The L2 HT(x,y) term requires that the sum of the transverse energy of jets with $p_{T}>y$ $\mathrm{GeV}$ is greater than $x \mathrm{GeV}$. The $z_{P V}$ term is a requirement on the distance of the primary vertex from the centre of the detector (measured along the beam axis), and the $\operatorname{Prob}_{b}(x)$ term indicates that the output of the L3 IP tagger must be less than $x$ for the event to fire the trigger. 
3.2 The $\phi b \bar{b}$ trigger conditions in the v15 and v16 trigger lists. The following requirements are made: the $\operatorname{CSWJT}(\mathrm{x}, \mathrm{y},|\eta|<\mathrm{z})$ term requires $\mathrm{x}$ L1 jets with energy greater than y GeV and with $|\eta|<\mathrm{z}$. The JT(x,y, $|\eta|<\mathrm{z})$ term requires $\mathrm{x}$ L2 or L3 jets with $p_{T}$ greater than $\mathrm{y}$ and $\mathrm{z}$ within $|\eta|<$ z. The L2 HT(x,y)term requires that the sum of the transverse energies of jets with $p_{T}>\mathrm{y} \mathrm{GeV}$ is greater than $\mathrm{x} G \mathrm{GeV}$, while the $\operatorname{SPHER}(\mathrm{x})$ term requires the event sphericity measured at L2 is greater than $\mathrm{x}$. The MJT(x,y) term is similar to the HT term in that it requires the total missing transverse energy $(\mathrm{MET})$ of jets with transverse energy $>\mathrm{y}$ $\mathrm{GeV}$ to be greater than $\mathrm{x} \mathrm{GeV}$. The $\operatorname{STTIP}(\mathrm{x}, \mathrm{y}, \mathrm{z})$ term requires $\mathrm{x}$ L2 STT tracks with impact parameter significance greater than or equal to $\mathrm{z}$, with a $\chi^{2}<\mathrm{y} .\left|z_{P V}\right|<35 \mathrm{~cm}$ is a requirement that the primary vertex reconstructed at L3 is within $35 \mathrm{~cm}$ of the centre of the detector, measured along the beam axis. The BTAG(0.4) term requires the L3 IP tagger to return a value less than 0.4 .

3.3 Number of events in each MC background sample. 59

3.4 The number of events in the p17 and p20 MC signal samples. 60

3.5 The number of events and relative fraction of events in data passing each cut in RunIIa.

3.6 The number of events and relative fraction of events in data passing each cut in RunIIb. Note that events with five or more jets are not included in this table.

3.7 Contributions of MC processes to the total MC multijet 3-jet background (RunIIa). The error on these values is of the order of $5 \%$.

3.8 Contributions of MC processes to the total MC multijet 3-jet background (RunIIb). The error on these values is of the order of $5 \%$.

3.9 Contributions of MC processes to the total MC multijet 4-jet background (RunIIb). The error on these values is of the order of $5 \%$.

5.1 Values of $\sigma \times$ branching ratio excluded with $95 \%$ confidence for $6.6 \mathrm{fb}^{-1}$ of DØ Run II data for different values of $m_{A}$. The excluded values of $\sigma \times B R$ for $\pm 1 \sigma$ and $\pm 2 \sigma$ around the expectation are also given. 


\section{Introduction}

This thesis describes a search for neutral supersymmetric Higgs bosons in the multijet final state at the $\mathrm{D} \varnothing$ experiment. The thesis is organised as follows:

- Chapter 1: The role of the Higgs boson in the Standard Model is discussed briefly, as well as the status of Higgs boson searches. The Minimal Supersymmetric Standard Model (MSSM) is introduced as an extension of the Standard Model, and differences between the MSSM and SM Higgs sectors are described.

- Chapter 2: The Tevatron accelerator and DØ experiment are described in this Chapter.

- Chapter 3: The search for neutral MSSM Higgs bosons in the $\phi b(b) \rightarrow b \bar{b} b(b)$ channel, using $6.6 \mathrm{fb}^{-1}$ of data from the $\mathrm{D} \varnothing$ detector, is introduced in this Chapter. The motivation for the search is given, and the data, background and signal samples are described.

- Chapter 4: A discriminant variable was used to separate signal and background events in order to increase the sensitivity of the analysis. A Multivariate Analysis (MVA) method was used to provide this discriminant, and this discriminant was used in the limit setting process. This Chapter gives an overview of some MVA techniques that were tested for this analysis. The MVA chosen to give the final discriminant, choice of inputs and MVA outputs are also given. In addition, a new event-based method was developed to replace the pair-based method previously used in this analysis.

- Chapter 5: This Chapter describes the process by which confidence limits were set on the Higgs boson cross-section multiplied by branching ratio, including a discussion of systematic uncertainties. Limits are also set on $\tan \beta$ for different MSSM scenarios. 
- Chapter 6: The final Chapter contains an overview of the work carried out and a discussion of possible extensions to the analysis.

In addition to the neutral MSSM Higgs boson search described in this thesis, the author has carried out studies to optimise high mass $\left(H \rightarrow W^{+} W^{-}\right)$and low mass $(Z H \rightarrow \nu \nu b \bar{b})$ Standard Model Higgs boson searches. These studies are both described in $\mathrm{D} \varnothing$ notes $[2,3]$, and the method developed by the author for the $H \rightarrow W^{+} W^{-}$analysis has been implemented in the high mass Standard Model Higgs boson searches [4, 5].

Chapters 3 to 5 describe the author's contribution to the search for neutral MSSM Higgs bosons in the multijet channel. The author was responsible for the implementation of a new event-based analysis method (replacing a jet pair-based method) and the development of the MVA technique, both described in Chapter 4. The author also calculated the confidence limits for this new method (Chapter 5), including the evaluation of systematic uncertainties.

The author has also worked on the Level 3 b-tagging trigger (described in Section 2.2.9.1), and has taken part in a study of the performance of the detector in increased luminosity conditions, described in Section 2.2.10.

Natural units are used throughout the thesis. 


\section{Chapter 1}

\section{Theory}

This Chapter gives an overview of the Standard Model (SM), with emphasis on the Higgs sector. The Standard Model is a mathematical description of the interactions of fundamental particles. An important component of the SM is the Higgs mechanism, which enables gauge bosons to have non-zero mass while preserving gauge symmetry (see Section 1.2). This mechanism also predicts the existence of an extra boson, the Higgs boson, which to date has not been observed.

Although the SM is hugely successful as a predictive model, there are many hints (both experimental and theoretical) that the SM is not a complete description of particle physics (Section 1.4). This has led to many extensions to the SM being proposed. One of these extensions, Supersymmetry (specifically the Minimal Supersymmetric Standard Model, or MSSM) is introduced in this Chapter. The MSSM solves many problems of the SM, and has perceived additional benefits (Section 1.4.1).

\section{$1.1 \quad$ Local Gauge Theories}

The behaviour of fundamental particles is well described by relativistic quantum mechanics [6]. The behaviour of electrons, for example, closely matches that predicted by the Dirac equation:

$$
\left(i \gamma^{\mu} \partial_{\mu}-m\right) \psi=0
$$

where $\psi$ is a four component spinor, $m$ is the electron mass, and $\gamma$ are the four Dirac matrices. This equation can be derived from a Lagrangian density, $\mathscr{L}$, as follows: 


$$
\mathscr{L}=\bar{\psi}\left(i \gamma^{\mu} \partial_{\mu}\right) \psi-m \bar{\psi} \psi
$$

where $\bar{\psi}=\psi^{\dagger} \gamma^{0}$. Note the $m \bar{\psi} \psi$ term, which dictates the electron's mass. The Lagrangian density will be referred to as the Lagrangian throughout.

One example of a symmetry transformation on the field $\psi$ is a phase change. The simplest type of transformation, multiplying by a $1 \times 1$ unitary $(\mathrm{U}(1))$ matrix, is given by:

$$
\psi \mapsto \psi^{\prime}=e^{i \theta(x)} \psi
$$

If $\theta(x)$ is constant with respect to the space-time co-ordinate, $x$, the symmetry is said to be global. When $\theta$ varies with the space-time co-ordinate, the symmetry is described as a local symmetry: the Lagrangian given in Equation (1.2) is not invariant under such a symmetry. Applying a phase change yields

$$
\mathscr{L} \mapsto \mathscr{L}^{\prime}=\mathscr{L}-\bar{\psi} \partial_{\mu} \theta(x) \psi
$$

The invariance of the Dirac Lagrangian can be retained by introducing a gauge term. This is achieved by replacing $\partial_{\mu}$ with a covariant derivative, $D_{\mu}=\partial_{\mu}+i q A_{\mu}$. $A_{\mu}$ is a 'gauge field', and is defined to transform in such a way that any changes due to a symmetry transformation in the rest of the Lagrangian are cancelled out by the gauge field term. In this example, the gauge field transformation

$$
A_{\mu} \mapsto A_{\mu}^{\prime}=A_{\mu}+\frac{1}{q} \partial_{\mu} \theta(x)
$$

maintains invariance under a local transformation. The invariant Dirac Lagrangian can then be written out in full as:

$$
\mathscr{L}=i \bar{\psi} \gamma_{\mu} \partial^{\mu}-m \bar{\psi} \psi-q \bar{\psi} \gamma_{\mu} A^{\mu} \psi
$$

The first two terms are identical to the non-invariant Dirac Lagrangian, while the third term describes interactions between the electron field and the gauge boson, with coupling strength $q$. This term therefore describes the electron-photon interaction. To fully describe the electromagnetic force, an additional term must be included to describe photon propagation:

$$
\mathscr{L}=i \bar{\psi} \gamma_{\mu} \partial^{\mu} \psi-m \bar{\psi} \psi-q \bar{\psi} \gamma_{\mu} A^{\mu}-\frac{1}{4} F_{\mu \nu} F^{\mu \nu}
$$

where 


$$
F_{\mu \nu}=\partial_{\mu} A_{\nu}-\partial_{\nu} A_{\mu}
$$

In the same way that photon fields are introduced to conserve $\mathrm{U}(1)$ symmetry, other symmetries are conserved by the introduction of further fields. The weak force is generated by the $\mathrm{SU}(2)$ symmetry, while the strong force is generated by $\mathrm{SU}(3)$ symmetry. These transformations take the form $\psi \mapsto \psi^{\prime}=e^{\tau_{i} \theta_{i}} \psi$, where $\tau_{i}$ is the $i$ th generator of the symmetry, and the Lagrangian is gauged by taking $D^{\mu}=\partial^{\mu}-i g \tau_{i} A_{i}^{\mu}$, where $\mathrm{g}$ is the coupling strength. The number of bosons is equal to the number of generators, so invariance under the $\mathrm{SU}(2)$ symmetry leads to the three weak boson fields, while invariance under $\mathrm{SU}(3)$ transformations leads to eight gluon fields.

The EM and weak forces can be expressed as components of a single force, the electroweak force $[7,8]$. This is generated by $\mathrm{SU}_{L}(2) \times \mathrm{U}_{Y}(1)$ symmetry $(\mathrm{SU}(2)$ is applied in weak isospin space, and the $\mathrm{U}(1)$ is in hypercharge space). To be invariant under this symmetry, the Lagrangian requires four gauge bosons, $A_{\mu}^{1}, A_{\mu}^{2}$ and $A_{\mu}^{3}$ from the $\mathrm{SU}(2)$ and $B_{\mu}$ from the $\mathrm{U}(1)$. The derivative for this model is

$$
D_{\mu}=\partial_{\mu}-\frac{i g}{2} \tau^{i} A_{\mu}^{i}-\frac{i q}{2} \mathbb{I} B_{\mu}
$$

where $q$ and $g$ are coupling constants and $\mathbb{I}$ is the $2 \times 2$ identity matrix. These fields are related to the physical weak and EM bosons by:

$$
\begin{gathered}
W_{\mu}^{ \pm}=\frac{1}{\sqrt{2}}\left(A_{\mu}^{1} \pm A_{\mu}^{2}\right) \\
Z_{\mu}^{0}=\frac{1}{\sqrt{g^{2}+q^{2}}}\left(q B_{\mu}-g A_{\mu}^{3}\right) \\
A_{\mu}=\frac{1}{\sqrt{g^{2}+q^{2}}}\left(q B_{\mu}+g A_{\mu}^{3}\right) .
\end{gathered}
$$

This model of the weak and EM forces describes the photon well; however, it does not allow for the masses of the weak bosons, which have been determined experimentally $[9,10]$. To include this mass, a term of the form $m_{b} A^{\mu} A_{\mu}$ would be required, where $m_{b}$ is the mass of the gauge boson. However, as the $A_{\mu}$ field follows the transformation given in Equation (1.5), this term would transform as:

$$
m_{b} A^{\mu} A_{\mu} \mapsto m_{b} A^{\prime \mu} A_{\mu}^{\prime}=m_{b} A^{\mu} A_{\mu}+\frac{m_{b}}{q}\left[\partial^{\mu} \theta A^{\mu}+A_{\mu} \partial_{\mu} \theta+\frac{1}{q}\left(\partial^{\mu} \theta\right)\left(\partial_{\mu} \theta\right)\right]
$$

and so is not invariant under the gauge transformation. This system of Lagrangians, therefore, is not able to describe observations from data. A method of resolving this issue is described in the next section. 


\subsection{The Higgs Mechanism}

In the Lagrangians considered above, the lowest possible energy state (the vacuum energy level) occurs at $\psi=0$, and the Lagrangians are all symmetric about this point. The Higgs mechanism $[12,13,14]$ introduces a scalar field, $\Phi$, described by a Lagrangian that is not necessarily symmetric about the vacuum.

$$
\begin{gathered}
\mathscr{L}=\frac{1}{2} D_{\mu} \Phi D^{\mu} \Phi-V(\Phi) \\
V(\Phi)=\frac{\mu^{2}}{2} \Phi^{*} \Phi+\frac{\lambda}{4}\left(\Phi^{*} \Phi\right)^{2}
\end{gathered}
$$

The potential, $V(\Phi)$, is plotted in Figure 1.1 for the cases $\mu^{2}>0$ and $\mu^{2}<0$. In the first case, $\mu$ may be interpreted as a physical mass, and there is a single value of $\Phi, 0$, at which $V$ is a minimum. At this point, the energy of the system is at its lowest, i.e. the vacuum energy. The value of $\Phi$ at which $V(\Phi)$ is at a minimum is known as the vacuum expectation value (VEV) of the system.

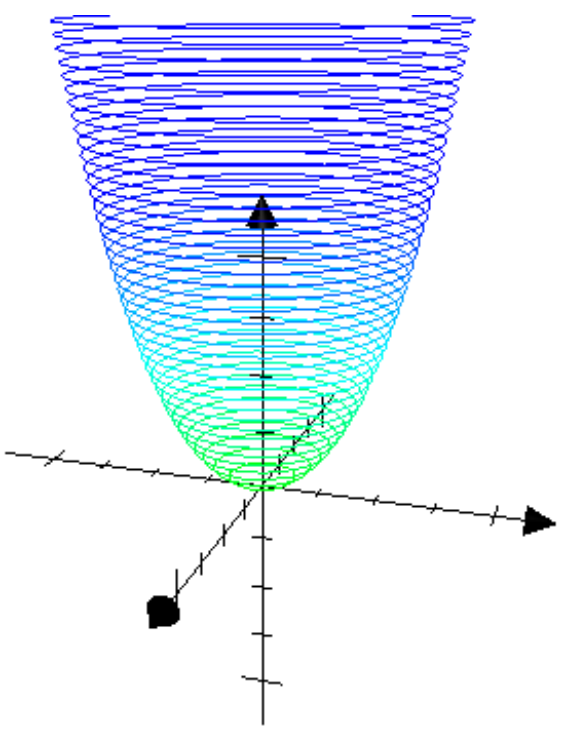

(a)

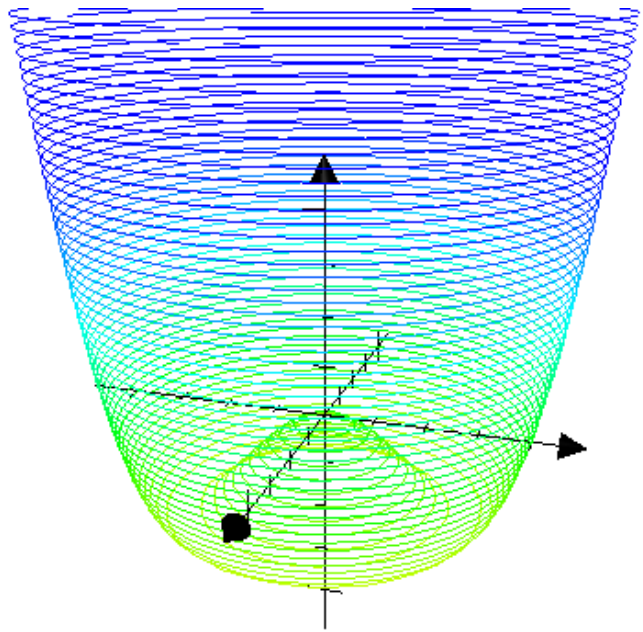

(b)

Figure 1.1: The potential $V$, defined in Equation (1.13), as a function of $\Phi$, for the case (a) $\mu^{2}>0$ and (b) $\mu^{2}<0$. In case (a), $\mu$ is a physical mass and the vacuum expectation value of $\Phi$ is 0 , while in case (b) there is a continuous range of VEVs.

In the case $\mu^{2}<0$, there is no single $\mathrm{VEV}$ for $\Phi$ : there is a continuous range of alternative vacua the system may occupy. Once the system occupies one of these states, the symmetry is said to be broken.

The symmetry of $V(\Phi)$ makes it more convenient to express $\Phi$ in terms of two scalar fields: 


$$
\Phi=\rho e^{i \eta}
$$

By taking perturbations around $v$, the value of $\rho$ that minimises $\mathrm{V}$, a particle $\rho^{\prime}=\rho+v$ may be defined, such that the $\rho^{\prime}$ boson has a physical mass term, $\frac{\mu^{2}}{2} \rho^{\prime 2}$, while the $\eta$ boson has no mass term. This massless boson is called a Goldstone boson. In general, the number of Goldstone bosons is equal to the number of generators of the broken symmetry (or symmetries) [11].

In terms of $\rho$ and $\eta$, the first part of the Lagrangian in Equation (1.12) becomes

$$
\mathscr{L}=\frac{1}{2}\left(D_{\mu} \rho-i\left(D_{\mu} \eta\right) \rho\right)\left(D^{\mu} \rho+i\left(D^{\mu} \eta\right) \rho\right)-V\left(\rho e^{i \eta}\right)
$$

Expanding the derivatives $D_{\mu}$ yields

$$
\mathscr{L}=\frac{1}{2}\left(\partial_{\mu} \rho-i q \mathscr{A}_{\mu} \rho\right)\left(\partial^{\mu} \rho+i q \mathscr{A}^{\mu} \rho\right)-V\left(\rho e^{i \eta}\right)
$$

where the Goldstone boson term, $\eta$, has been absorbed into the $A_{\mu}$ term, leading to a new boson term, defined by $\mathscr{A}_{\mu}=A_{\mu}-\frac{1}{q} \partial_{\mu} \eta$. Using the substitution $\rho^{\prime}=\rho+v$ once again, it can be shown that the $\mathscr{A}_{\mu}$ boson has a mass term $\frac{q^{2} v^{2}}{2} \mathscr{A}_{\mu} \mathscr{A}^{\mu}$. This mass term is invariant: by grouping the Goldstone term with the gauge boson, the changes due to a $\mathrm{U}(1)$ symmetry transformation cancel. The remaining term from $\Phi, \rho^{\prime}$, indicates the presence of an extra boson, known as the Higgs boson. This interacts with the $\mathscr{A}$ boson via the term $\frac{m_{\mathscr{A}}^{2}}{v} \rho^{\prime} \mathscr{A}_{\mu} \mathscr{A}^{\mu}$, where $m_{\mathscr{A}}=q v$. Therefore the coupling of the Higgs boson to the gauge boson is proportional to the mass of the gauge boson.

To summarise, it is possible to produce an invariant massive gauge boson term by breaking the symmetry of the system and absorbing the resulting Goldstone boson into the gauge field. This mechanism allows the weak bosons to have nonzero mass in the Standard Model, as described in the next section, but also predicts the existence of a scalar boson which couples to the weak bosons in proportion to their mass.

\subsubsection{The Higgs Mechanism in the Standard Model}

In the electroweak model, there are three massive gauge bosons: the $W^{+}, W^{-}$and $Z^{0}$. It follows that three Goldstone terms must be created, which in turn requires the symmetries due to three of the four generators of $\mathrm{SU}(2) \times \mathrm{U}(1)$ to be broken. The Higgs field required for this Lagrangian therefore takes the form of a complex doublet: 


$$
\Phi=\left(\begin{array}{l}
\phi_{1}+i \phi_{2} \\
\phi_{3}+i \phi_{4}
\end{array}\right)
$$

Applying the Higgs mechanism to the electroweak Lagrangian gives similar results to the $\mathrm{U}(1)$ case: the $W^{ \pm}$bosons gain a mass of $\frac{g v}{2}$ (where $v$ is once again the VEV of the Higgs field), the $Z^{0}$ boson gains a mass of $\frac{v \sqrt{g^{2}+q^{2}}}{2}$, and the photon remains massless. Three of the four fields in the Higgs doublet of Equation (1.17) are Goldstone fields, and are absorbed into the weak boson terms. The remaining field survives as a Higgs boson with mass $2 \lambda v^{2}$.

So far, only interactions between the Higgs boson, $H$, and gauge bosons have been considered. The interactions of the Higgs field with matter particles are described by the Yukawa term:

$$
\mathscr{L}_{\text {Yukawa }}=-G_{f}\left(\bar{\Psi}_{L} \Phi \Psi_{R}+\bar{\Psi}_{R} \Phi^{\dagger} \Psi_{L}\right)
$$

where the $L$ and $R$ subscripts denote left- and right-handed fermions, and $G_{f}$ are the Yukawa couplings. The electron term, for example, is

$$
\mathscr{L}_{\text {Yukawa }}=-\frac{G_{e} v}{\sqrt{2}}\left(\bar{e}_{L} e_{R}+\bar{e}_{R} e_{L}\right)-\frac{G_{e}}{\sqrt{2}}\left(\bar{e}_{L} e_{R}+\bar{e}_{R} e_{L}\right) H
$$

The first term gives the mass of the electron as $m_{e}=\frac{G_{e} v}{\sqrt{2}}$, while the second term is the coupling to the Higgs boson. This indicates that the Higgs coupling to fermions is proportional to the fermion mass, similar to the coupling with the gauge bosons. For this reason, the Higgs boson is predicted to typically decay most often to the highest mass particle that kinematics allow. For example, if the Higgs boson mass, $m_{H}$, is $120 \mathrm{GeV}$, the Higgs is most likely to decay to a $b \bar{b}$ pair (coupling to $W^{+} W^{-}$, $Z^{0} Z^{0}$ and $t \bar{t}$ is suppressed at this value of $m_{H}$, as $120 \mathrm{GeV}<2 m_{W}<2 m_{Z}<2 m_{t}$ ).

Many aspects of the Standard Model have been experimentally verified. The existence of the Higgs boson, however, has not been confirmed. The Higgs boson plays an integral part in the SM, and searches for the Higgs boson, and attempts to determine its properties, are a key test of the Standard Model. The constraints on these properties are discussed in the next section.

\subsection{Constraints on the Standard Model Higgs Bo- son}

Although the Higgs boson has not been observed, the allowed range of its mass (and so coupling to other particles) can be determined through theoretical considerations and experimental evidence. Some of these constraints are presented in this Section. 


\subsubsection{Theoretical Constraints}

The Higgs mechanism requires the constant $\lambda$ in Equation (1.13) to be finite and positive [15]. If the SM is not taken to be a complete model of particle physics (see Section 1.4), this requirement can be replaced with the less stringent condition that $\lambda$ must be finite and positive below some energy cutoff $\Lambda$, with new physics taking effect above $\Lambda$. The value of $\lambda$ is given by

$$
\lambda=\frac{m_{H}^{2}}{2 v^{2}}=\frac{G_{F} m_{H}^{2}}{\sqrt{2}}
$$

Where $G_{F}$ is the Fermi coupling constant. As the energy scale increases, higher order processes must be included in the calculation of $G_{F}$, and so the Fermi constant increases ('runs') with energy scale, so $\lambda$ also varies with $\Lambda$. The above requirements on the value of $\lambda$ translate directly to constraints on the Higgs boson mass which vary with energy scale.

The cross sections for Higgs decays to weak bosons contain corrections proportional to $\lambda$. For high values of $\lambda$, these cross-sections become divergent. Conversely, for small values of $\lambda$ (and so $m_{H}$ ), the coupling to the top quark can become negative. These considerations lead to upper and lower theoretical bounds on the Higgs mass: these are shown in Figure 1.2.

At the Planck scale, i.e. $\Lambda=10^{19} \mathrm{GeV}$, a Higgs mass of $160-170 \mathrm{GeV}$ fulfils the above requirements. If $\Lambda$ is closer to $1 \mathrm{TeV}$, the range is approximately $85<m_{H}<$ $420 \mathrm{GeV}$.

\subsubsection{Direct Searches}

Direct searches for the Higgs boson have been able to rule out its existence for certain mass hypotheses [16]. The four LEP experiments (ALEPH [17], DELPHI [18], L3 [19] and OPAL [20]) collected a total of $2.46 \mathrm{fb}^{-1}$ of data from electronpositron collisions at a centre of mass energy of $189-209 \mathrm{GeV}$ [21]. At the available luminosity, the Higgs boson was only detectable if the process $e^{+} e^{-} \rightarrow H Z$ was kinematically accessible, so the experiments were sensitive to Higgs bosons with $m_{H}$ up to $119 \mathrm{GeV}$. The dominant Higgs decays in this mass range are to $b \bar{b}$ and $\tau^{+} \tau^{-}$: the former were searched for in four jet $(H \rightarrow b \bar{b}, Z \rightarrow q \bar{q})$, missing energy $(H \rightarrow b \bar{b}$, $Z \rightarrow \nu \bar{\nu})$, and lepton $\left(H \rightarrow b \bar{b}, Z \rightarrow \ell^{+} \ell^{-}\right)$events, while only the hadronic signature $\left(H \rightarrow \tau^{+} \tau^{-}, Z \rightarrow q \bar{q}\right)$ was examined for the latter. By setting confidence limits on the cross-section for these processes, the LEP collaborations were able to exclude a Higgs mass range $m_{H}<114.4 \mathrm{GeV}$. 


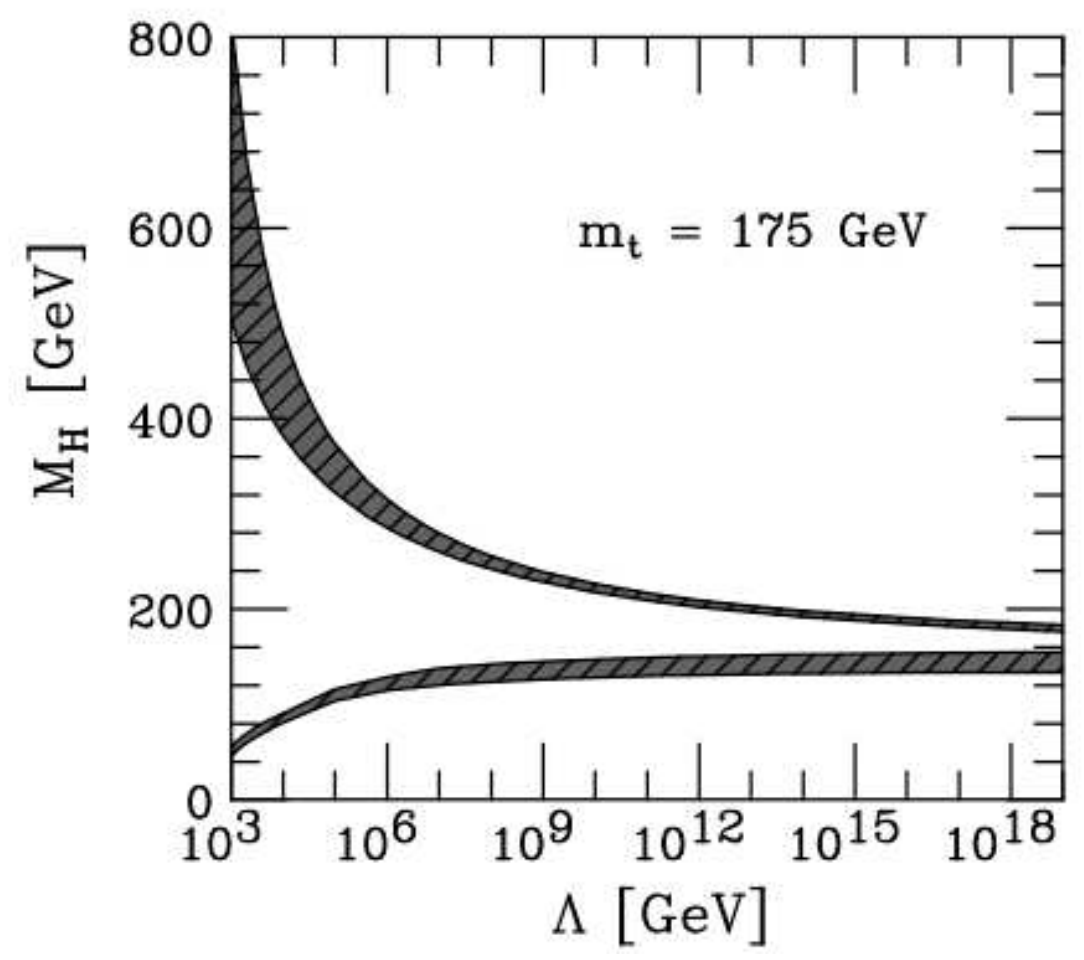

Figure 1.2: Theoretical constraints on the Higgs boson mass as a function of energy scale $\Lambda$ [15]. The parameter space above (below) the upper (lower) grey band is excluded.

In addition, the CDF [22] and DØ [23] experiments at the Fermilab Tevatron have been able to exclude a Higgs mass of $158<m_{H}<175 \mathrm{GeV}$ to $95 \%$ confidence [4]. The Tevatron has a centre of mass energy of $1.96 \mathrm{TeV}$, so the range of accessible Higgs masses is extended to $200 \mathrm{GeV}$ (the upper limit on Higgs mass from indirect searches: see 1.3.3). The Tevatron SM Higgs searches are most sensitive to the $H \rightarrow W^{+} W^{-}$channel, which has led to exclusion around $m_{H}=2 m_{W}$. The Higgs mass range excluded by the Tevatron is shown in the Figure 1.3.

\subsubsection{Indirect Measurements}

As well as excluding potential values of the Higgs mass by direct searches, the dependence of the electroweak interactions on the Higgs mass can be used to set indirect limits on the Higgs mass. The Higgs mass may be constrained by calculating the EW parameters for a range of Higgs masses, and calculating the $\chi^{2}$ between the predicted and measured values. The minimum possible $\chi^{2}$ occurs at $m_{H}=85 \mathrm{GeV}$. The $\chi^{2}$ calculation also places an upper limit of $158 \mathrm{GeV}$ on the Higgs boson mass with $95 \%$ confidence. The $\Delta \chi^{2}\left(\chi^{2}\left(m_{H}\right)-\chi_{\min }^{2}\right)$ is plotted as a function of Higgs mass in Figure 1.4. This indicates that the range of possible Higgs boson masses favoured by the SM is accessible at the Tevatron. 


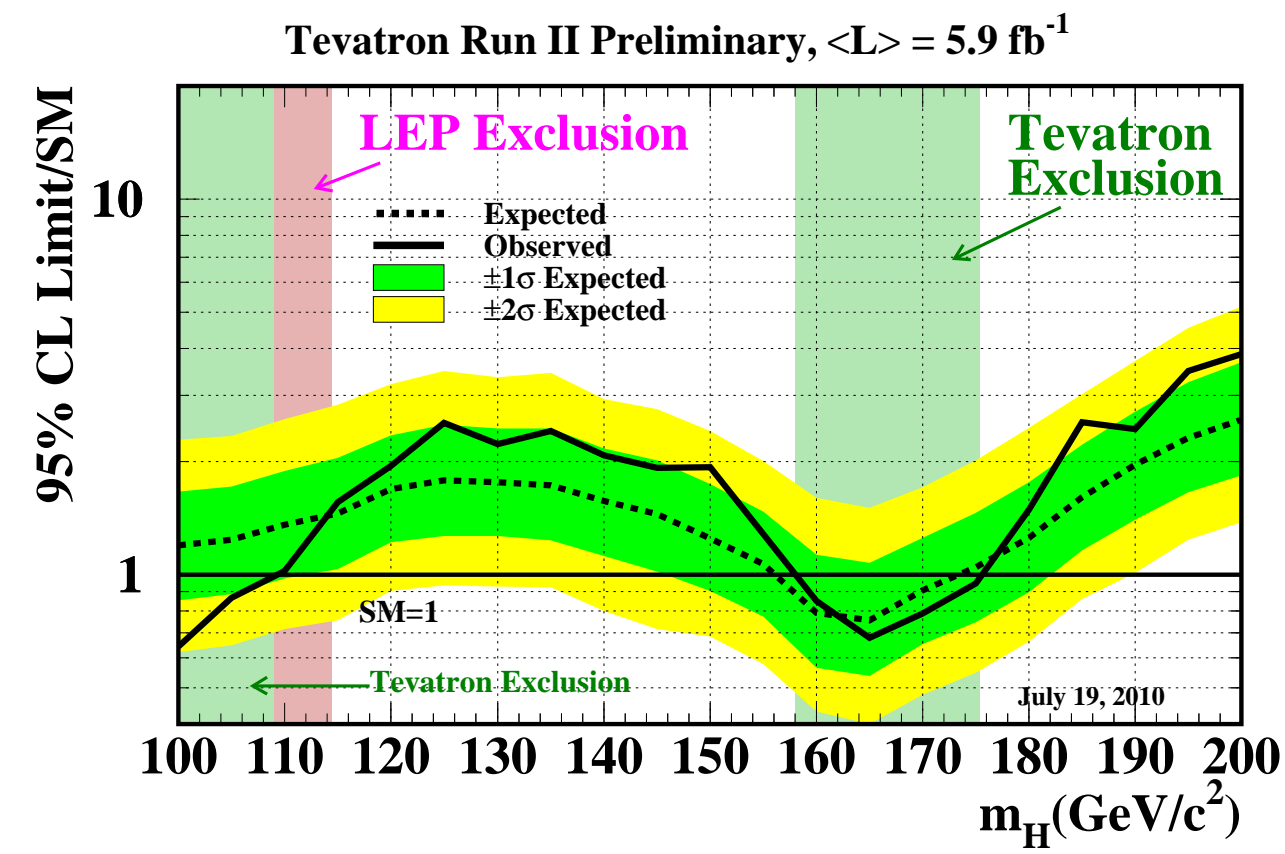

Figure 1.3: Combined confidence limits on SM Higgs production from the Tevatron experiments [24]. The plot shows the value of $\sigma \times B R$ excluded to $95 \%$ confidence divided by the theoretical value as a function of Higgs boson mass. Where the solid black (observed limit) line is below one, the SM Higgs boson is excluded to $95 \%$ confidence.

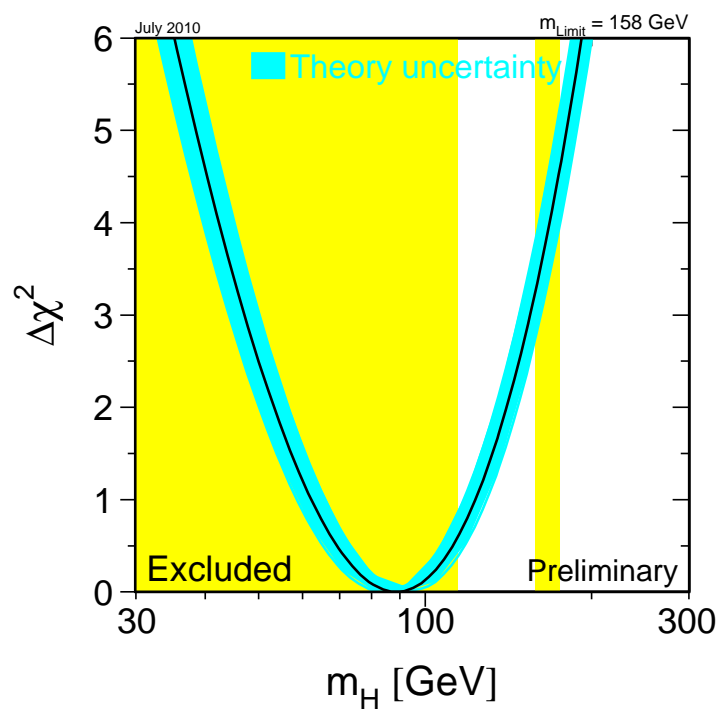

Figure 1.4: $\Delta \chi^{2}$ for fits of EW parameters for different Higgs masses, where $\Delta \chi^{2}=\chi^{2}\left(m_{H}\right)-\chi_{m i n}^{2}$ [25]. The best fit occurs at $85 \mathrm{GeV}$. 


\subsection{Beyond the Standard Model}

Although the Standard Model has been confirmed extensively, there are reasons to believe that it is not a complete model of particle physics. Indications that there might be physics beyond the Standard Model include:

- The Standard Model requires the Higgs boson to have a mass below $1 \mathrm{TeV}$. At higher energy scales, loop corrections in the Higgs mass calculation increase the mass beyond this scale (this is known as the 'Hierarchy Problem'). This may be prevented by adjusting the parameters, but these adjustments would be very large. A theory which avoids the Hierarchy Problem without the need for 'tuning' is preferential from a theoretical standpoint.

- The coupling constants of the EM, weak and strong forces run with energy scale. In a Grand Unified Theory, the running coupling constants must converge to a single value at high energy scale (around $10^{15} \mathrm{GeV}$ ) [26]. This convergence is not predicted by the SM. The running of the constants is plotted in Figure 1.5.

- A large proportion of the universe is thought to be made up of dark matter: this is not included in the Standard Model.

- Although the Standard Model correctly describes and predicts many observed phenomena, there are many aspects which are unexplained. For example, the Standard Model does explain why there is more than one generation of matter. The Standard Model also lacks an explanation for gravity.

A number of extensions to the SM have been proposed to solve some of these problems. The remainder of this Chapter will focus on one such extension, Supersymmetry.

\subsubsection{Supersymmetry and the MSSM Higgs Sector}

Supersymmetry (SUSY) [27, 28] is a proposed extra symmetry of particle physics which transforms fermions to bosons and vice versa:

$$
Q \Phi=\Psi, \quad Q \Psi=\Phi
$$

where $Q$ is a Supersymmetric operator, $\Psi$ is a fermionic field and $\Phi$ is a bosonic field. For example, the electron, $e$, has a proposed superpartner known as the 

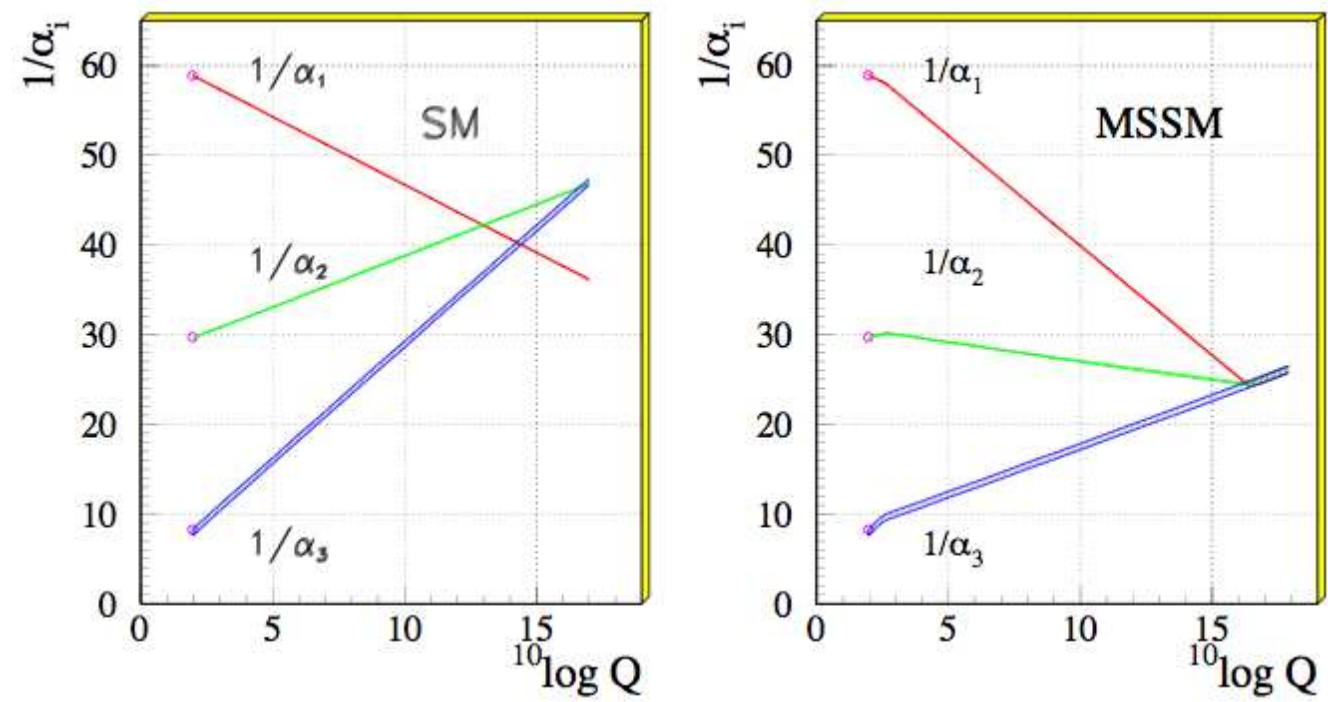

Figure 1.5: The inverse of $\alpha_{1}, \alpha_{2}$ and $\alpha_{3}$ (proportional to the square of the $\mathrm{U}(1), \mathrm{SU}(2)$ and $\mathrm{SU}(3)$ coupling constants respectively) for the SM (left) and MSSM (right), as a function of energy scale $\mathrm{Q}$ in $\mathrm{GeV}[26]$.

selectron, denoted as e e. Such a symmetry requires that fermionic partner fields exist for the bosonic fields, and vice versa. Supersymmetry has a number of perceived advantages over the Standard Model:

- When calculating the Higgs mass to higher orders, fermionic loops carry the opposite sign to bosonic loops. The loops for particles cancel with their superpartners to some extent (the cancellation is not exact, as particles and their super-partners have different masses due to SUSY-breaking). This reduces the level of tuning required, thus reducing the scale of the Hierarchy Problem.

- The running coupling constants for the EM, weak and strong forces converge at the same point in the MSSM.

- Supersymmetric models contain candidate dark matter particles [29].

Much like the Standard Model, supersymmetric models require a Higgs field to give the weak bosons mass. The simplest supersymmetric extension to the SM, the Minimal Supersymmetric Standard Model (MSSM), requires two Higgs doublets $\Phi_{u}$ and $\Phi_{d}$, which interact with up- and down-type quarks respectively. The VEVs for these doublets are denoted $v_{u}$ and $v_{d}$.

Three of the eight scalar fields from these doublets are absorbed into the gauge boson terms to produce massive gauge bosons. In a similar manner to the SM, the mass of the $W$ bosons is related to the VEVs by : 


$$
m_{W}^{2}=\frac{g^{2}}{2}\left(v_{u}^{2}+v_{d}^{2}\right)
$$

Hence the values of $v_{u}$ and $v_{d}$ are constrained, and can be combined into a single free parameter, $\tan \beta$ :

$$
\tan \beta=\frac{v_{d}}{v_{u}}
$$

The remaining five fields from the complex doublets remain as Higgs bosons. These are denoted $H, h, A, H^{+}$and $H^{-} . H^{+}$and $H^{-}$are charged scalar bosons, the $A$ is a neutral pseudoscalar boson, and the $h$ and $H$ are neutral scalar bosons. The masses of the neutral bosons (to tree level) are related by [30]:

$$
m_{H, h}^{2}=\frac{1}{2}\left(m_{A}^{2}+m_{Z}^{2} \mp \sqrt{\left(m_{A}^{2}+m_{Z}^{2}\right)^{2}-4 m_{A}^{2} m_{Z}^{2} \cos ^{2} 2 \beta}\right)
$$

and the mass of the charged Higgs bosons is given by:

$$
m_{H^{ \pm}}^{2}=m_{W}^{2}+m_{A}^{2}
$$

Up- and down-type quarks interact with only one MSSM Higgs doublet, and the coupling constant is proportional to the VEV of the doublet. This change in the coupling can be expressed as a correction to the couplings to the SM Higgs. The corrections for each neutral Higgs boson interaction with quarks are given in Table 1.1. The mixing angle, $\alpha$, used in Table 1.1 is given by

$$
\cos ^{2}(\beta-\alpha)=\frac{m_{h}^{2}\left(m_{Z}^{2}-m_{h}^{2}\right)}{m_{A}^{2}\left(m_{H}^{2}-m_{h}^{2}\right)}
$$

\begin{tabular}{|c|ccc|}
\hline Quark type & $h$ & $H$ & $A$ \\
\hline$u$ & $\frac{\cos \alpha}{\sin \beta}$ & $\frac{\sin \alpha}{\sin \beta}$ & $\cot \beta$ \\
$d$ & $\frac{-\sin \alpha}{\cos \beta}$ & $\frac{\cos \alpha}{\cos \beta}$ & $\tan \beta$ \\
\hline
\end{tabular}

Table 1.1: The MSSM enhancements over the SM for various neutral Higgs boson-quark interactions. $\alpha$ is a mixing angle, describing interactions between the two Higgs doublets [31].

At tree level, the MSSM Higgs boson masses (Equations (1.24) and (1.25)) and coupling constants (Table 1.1) can be expressed in terms two parameters: these are customarily chosen as $m_{A}$ and $\tan \beta$, so it is common for MSSM Higgs boson searches set limits on these values.

The masses of the up- and down-type quarks are related to the Higgs coupling, and the ratio of the top and bottom quark masses suggests that $\tan \beta$ is relatively 
high, around 35 [32]. A high $\tan \beta$ value has many implications for the properties of the MSSM Higgs sector. The enhancement in Table 1.1 suggests that the production of $A$ bosons in association with $b$-quarks in the MSSM is more common than in the SM by a factor of $\tan ^{2} \beta$.

As $\tan \beta$ increases, the value of $\cos ^{2} 2 \beta$ in Equation (1.24) approaches 1 asymptotically. The mass equations become:

$$
m_{H} \approx m_{A}, m_{h} \approx m_{h}(\max ) \quad\left(m_{A}>m_{h}(\max )\right)
$$

or

$$
m_{h} \approx m_{A}, m_{H} \approx m_{h}(\max ) \quad\left(m_{A}<m_{h}(\max )\right)
$$

where $m_{h}(\max )$ is equal to $m_{Z}$ at tree level: when the effects of virtual loops are taken into account, corrections from the stop sector increase the value of $m_{h}(\max )$ to roughly $130 \mathrm{GeV}$. It can be seen that at least two of the three neutral MSSM Higgs bosons display a mass degeneracy at high $\tan \beta$ : this is illustrated at leading order in Figure 1.6.

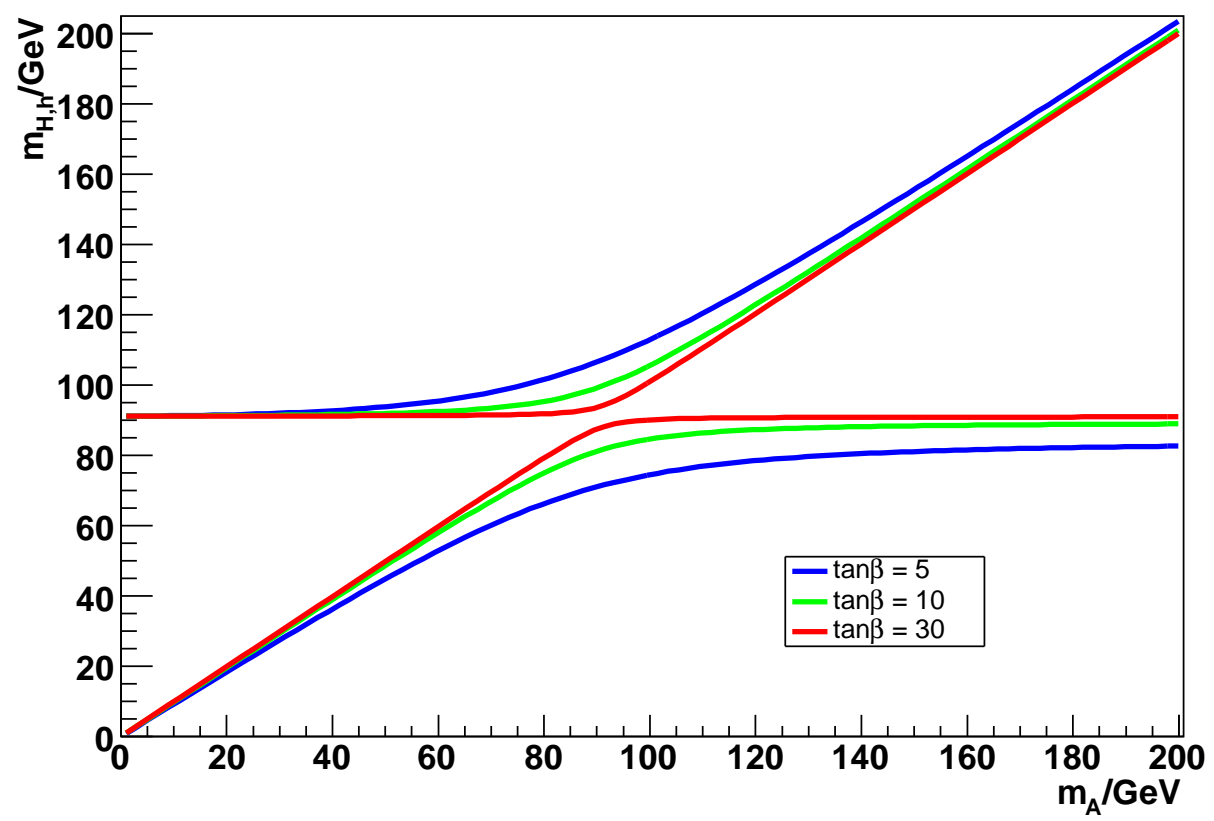

Figure 1.6: The tree level masses of the $H$ (top) and $h$ (bottom) bosons as a function of $m_{A}$ for different $\tan \beta$ values, given by Equation (1.24). As $\tan \beta$ increases, a mass degeneracy where $m_{h} \approx m_{A}$ or $m_{H} \approx m_{A}$ occurs. When radiative corrections are applied, the maximum value of $m_{h}$ increases from $m_{Z}$ to roughly $130 \mathrm{GeV}$ 
Additionally, substituting the mass values in Equations (1.27) and (1.28) into Equation (1.26) at high $\tan \beta$ gives $\sin \alpha \approx 0$ and $\sin \alpha \approx 1$ respectively. This leads to the coupling of down-type quarks to the boson that shares mass with the $A$ boson also being enhanced by $\tan \beta$. Therefore, this mass degeneracy increases the rate of neutral Higgs bosons decaying to $b$-quarks by a factor of two. For these reasons, the frequency of $\phi b \rightarrow b b b$ events, where $\phi$ denotes any one of $H, h$ and $A$, is thought to be greatly enhanced in the MSSM in comparison with the SM, making this a favourable channel to search for low mass neutral MSSM Higgs bosons at high $\tan \beta$ (Higgs boson decays to $\tau$ leptons show a similar enhancement). This large enhancement to the cross-section means that the Tevatron experiments are sensitive to neutral MSSM Higgs bosons.

\subsubsection{Constraints on the Neutral MSSM Higgs Bosons}

The four LEP experiments searched for neutral MSSM Higgs bosons in the same channels as used in the SM Higgs boson search, with the addition of Higgs pair production searches (i.e. $\phi \phi \rightarrow b \bar{b} b \bar{b}, \phi \phi \rightarrow \tau^{+} \tau^{-} \tau^{+} \tau^{-}$and $\phi \phi \rightarrow b \bar{b} \tau^{+} \tau^{-}$). By combining the results of these searches, the LEP experiments were able to exclude the region $m_{A}<93 \mathrm{GeV}$ for all values of $\tan \beta$, assuming the MSSM Higgs sector conserves CP (no lower limits on Higgs boson masses could be set for $\mathrm{CP}$ violating scenarios for all $\tan \beta$, but large regions of parameter space were excluded) [33].

Due to the higher centre of mass energy, the CDF and DØ experiments have been able to extend sensitivity to neutral MSSM Higgs bosons to a greater range of $m_{A}$. Again, these experiments have set limits using $\phi \rightarrow \tau \tau[34,35,36]$ and $\phi \rightarrow b b$ decays $[37,1]$. The analysis presented in this thesis (Chapters 3 to 5) uses triple $b$-jet events from $6.6 \mathrm{fb}^{-1}$ of data collected by the $\mathrm{D} \varnothing$ detector (Chapter 2) to set expected confidence limits on $\tan \beta$ for values of $m_{A}$ from $90 \mathrm{GeV}$ to $300 \mathrm{GeV}$. 


\section{Chapter 2}

\section{The DØ Experiment}

\subsection{The Tevatron}

The Tevatron is a $1.96 \mathrm{TeV} p \bar{p}$ collider located within the Fermi National Accelerator Laboratory (FNAL, or Fermilab) complex in Illinois [38]. The colliding particles are accelerated in a succession of smaller accelerators, illustrated in Figure 2.1. The Tevatron has a radius of one kilometre, and produces collisions at two points: the $\mathrm{CDF}$ and D $\varnothing$ detector collision halls. The Tevatron's period of operation can be divided into a series of 'Runs': for example, Run I took place from 1992 to 1996, and Run II began in 2001 and is still in progress. The differences between Runs I and II are given in Table 2.1. Both detectors were refitted at the end of Run I to prepare for the increased luminosities of Run II. Run II can be further divided into Run IIa and Run IIb: the DØ detector was again modified between these periods. At present, the experiment is scheduled to run until the end of 2011, with a target delivered integrated luminosity of $12 \mathrm{fb}^{-1}$. There is a possibility, however, that Run II will continue to the end of 2014, in which case the target delivered integrated luminosity is $20 \mathrm{fb}^{-1}$ [39]. The analysis in this thesis uses $6.6 \mathrm{fb}^{-1}$ of D $\varnothing$ data (1 $\mathrm{fb}^{-1}$ from RunIIa and $5.6 \mathrm{fb}^{-1}$ from Run IIb).

\subsection{The DØ Detector}

The D $\varnothing$ detector consists of a series of subdetectors arranged in concentric layers centred around the interaction point, as shown in Figure 2.2. The innermost detector is the tracker, surrounded by a solenoid, a calorimeter and muon systems. These are described in sections 2.2.1 to 2.2.5: full details are given in [41]. 


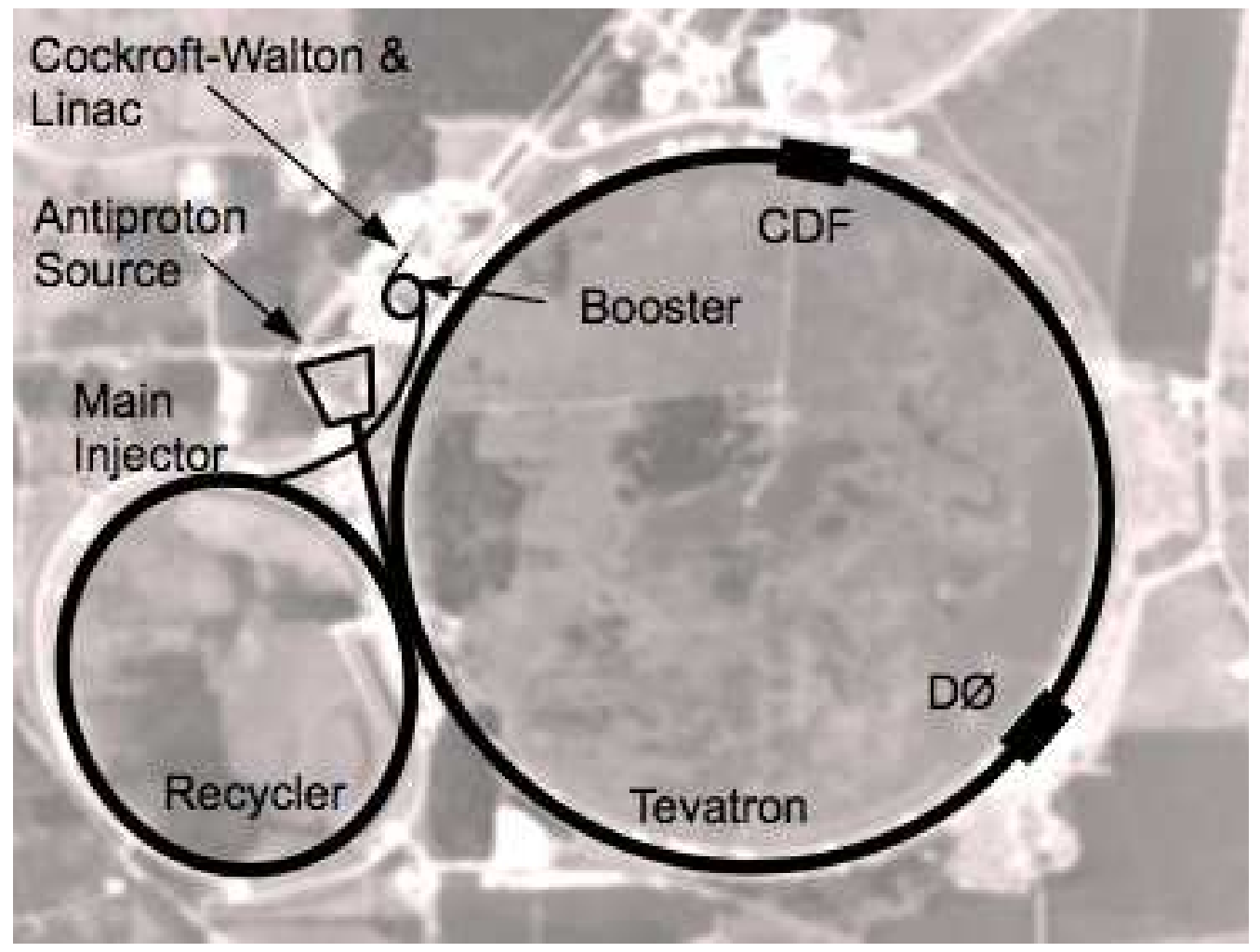

Figure 2.1: The Tevatron and accelerator chain. Hydrogen atoms are ionised using an electric field generated using a Cockroft-Walton voltage multiplier. The resulting protons line the caesium cathode, and can combine with electrons from the caesium to form $\mathrm{H}^{-}$ions (caesium is used because of its low work function). These are repelled by the cathode and directed through a graphite target to strip away the electrons. The resulting protons are transferred to the booster, which raises their energy to $8 \mathrm{GeV}$, and then to the Main Injector. Some protons are directed to a nickel target to produce antiprotons. The protons and antiprotons are accelerated to energies of $150 \mathrm{GeV}$ before being injected into the Tevatron (aerial photograph from [40]). 


\begin{tabular}{|c|c|c|c|}
\hline & Run I & Run IIa & Run IIb \\
\hline$p \bar{p}$ Energy $(\mathrm{GeV})$ & 900 & 980 & 980 \\
Proton bunches & 6 & 36 & 103 \\
Protons/bunch & $2.3 \times 10^{11}$ & $2.7 \times 10^{11}$ & $2.7 \times 10^{11}$ \\
Antiproton bunches & 6 & 36 & 103 \\
Antiprotons/bunch & $5.5 \times 10^{10}$ & $3.0 \times 10^{10}$ & $1.0 \times 10^{11}$ \\
Bunch spacing $(\mathrm{ns})$ & 3500 & 396 & 132 \\
Peak Luminosity $\left(\mathrm{cm}^{-2} \mathrm{~s}^{-1}\right)$ & $0.16 \times 10^{32}$ & $0.86 \times 10^{32}$ & $5.2 \times 10^{32}$ \\
Luminosity (pb $\left.{ }^{-1} / \mathrm{week}\right)$ & 3.2 & 17.3 & 105 \\
Interactions per crossing & 2.5 & 2.3 & 4.8 \\
\hline
\end{tabular}

Table 2.1: A summary of Tevatron properties in Run I and Run II [40].

The detector is described by a co-ordinate system defined as follows: the origin of the system is the centre of the detector and the z-axis is taken to be parallel to the beam pipe, with $\mathrm{z}$ increasing in the direction of travel of the protons. The y-axis is oriented vertically upwards, while the x-axis points to the west to give a righthanded co-ordinate system. Due to the symmetry of the detector, it is preferable to use $(\mathrm{r}, \theta, \phi)$ co-ordinates as opposed to $(\mathrm{x}, \mathrm{y}, \mathrm{z})$, where $\mathrm{r}$ is the distance of the object from the origin, $\theta$ is the polar angle and $\phi$ is the azimuthal angle. The polar angle is usually replaced by the pseudo-rapidity, $\eta$, given by $-\ln \left(\tan \left(\frac{\theta}{2}\right)\right)$. This quantity is approximately equal to the rapidity $\left(\frac{1}{2} \ln \left(\frac{E+p_{z}}{E-p_{z}}\right)\right)$ in the high energy limit.

\subsubsection{Tracking Detector}

The D $\varnothing$ tracking system is located outside of the beampipe, and within a 2 Tesla solenoidal magnet, as illustrated in Figure 2.3. The tracking system is designed to give tracking information within $|\eta|<3$. This allows measurement of momentum for charged particles and aids particle identification. The tracking detector consists of a silicon microstrip tracker and a scintillating fibre tracker.

\subsubsection{The Silicon Microstrip Tracker}

The Silicon Microstrip Tracker (SMT) is made up of silicon wafers arranged in a number of barrels and disks and held in place by a beryllium support. The SMT is illustrated in Figure 2.4. A description of the barrels and disks is given below. 


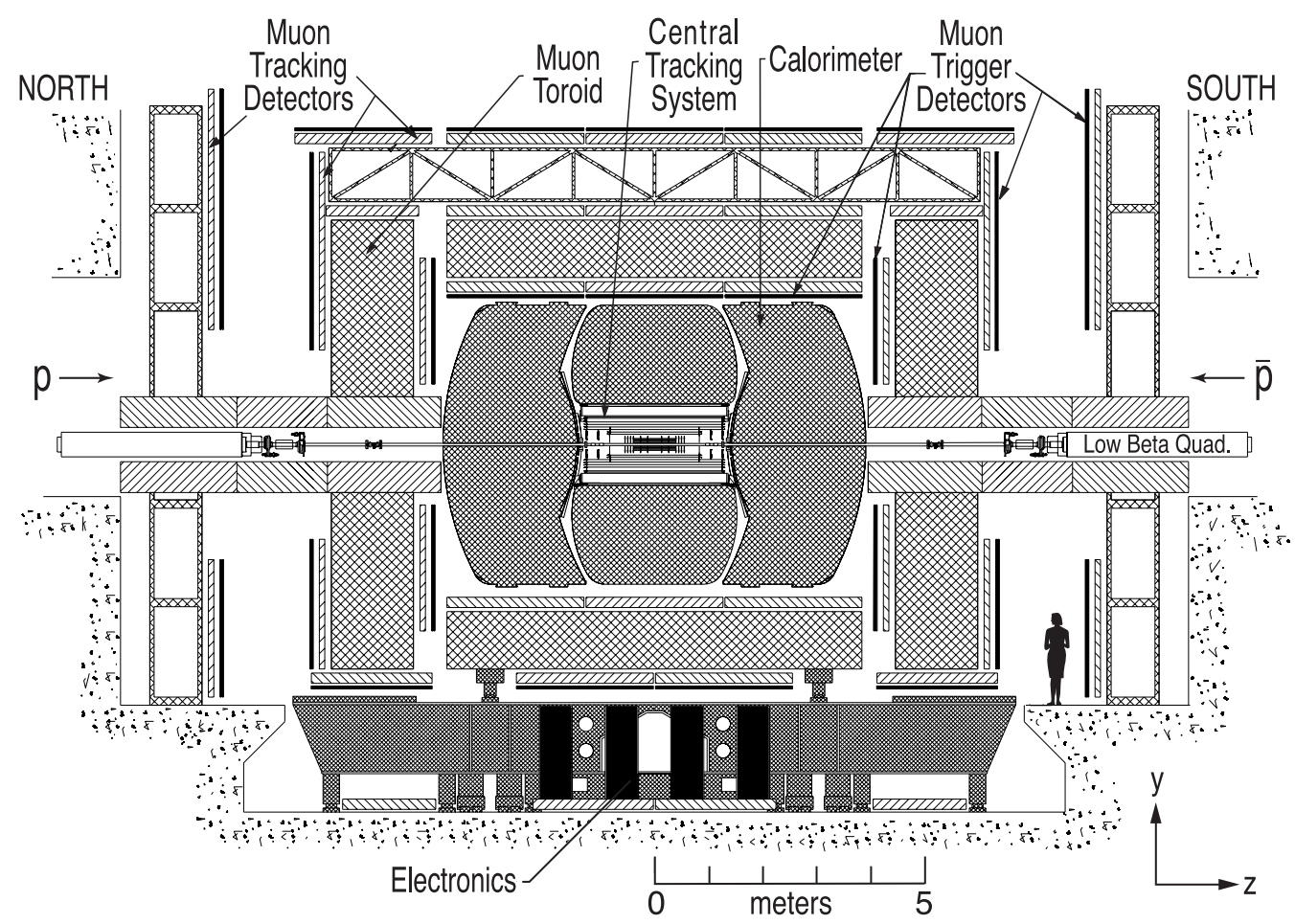

Figure 2.2: The full $\mathrm{D} \emptyset$ detector, including (from centre outwards) tracking, preshower, calorimeter and muon sub-detectors. On the diagram the $\mathrm{z}$ axis runs from left to right through the centre of the detector, and the y-axis runs from bottom to top [41].

- Barrel: The barrel sections of the SMT are intended to measure momentum in the transverse $(\mathrm{x}-\mathrm{y})$ plane. The $p \bar{p}$ interactions occur within a large range of the z-axis (within $\approx 26 \mathrm{~cm}$ of the origin), so the SMT barrel extends over $|z|<38 \mathrm{~cm}$. To this end, six barrel sections of length $12.4 \mathrm{~cm}$ are arranged about the origin. Each of these barrels contains five layers, zero to four, of groups of rectangular double-sided silicon detectors (ladders): each of the inner three (outer two) layers contains 12 (24) ladders, so there are 504 ladders in the SMT as a whole. The barrel sections cover a radius of $2.7 \mathrm{~cm}$ to $10.5 \mathrm{~cm}$. The innermost layer, layer-0, was added in the shutdown between RunIla and RunIIb to improve b-tagging resolution for low transverse momentum $\left(p_{T}\right)$ jets and to compensate for any loss of performance in the ageing silicon.

- F Disks: The twelve F Disks are oriented orthogonally to the beamline, and one is positioned between each pair of barrel sections (apart from the gap at $\mathrm{z}=0$ ). Three are positioned at each end of the whole SMT barrel. Each disk consists of twelve double-sided, wedge-shaped silicon detectors, arranged in two layers of six. 


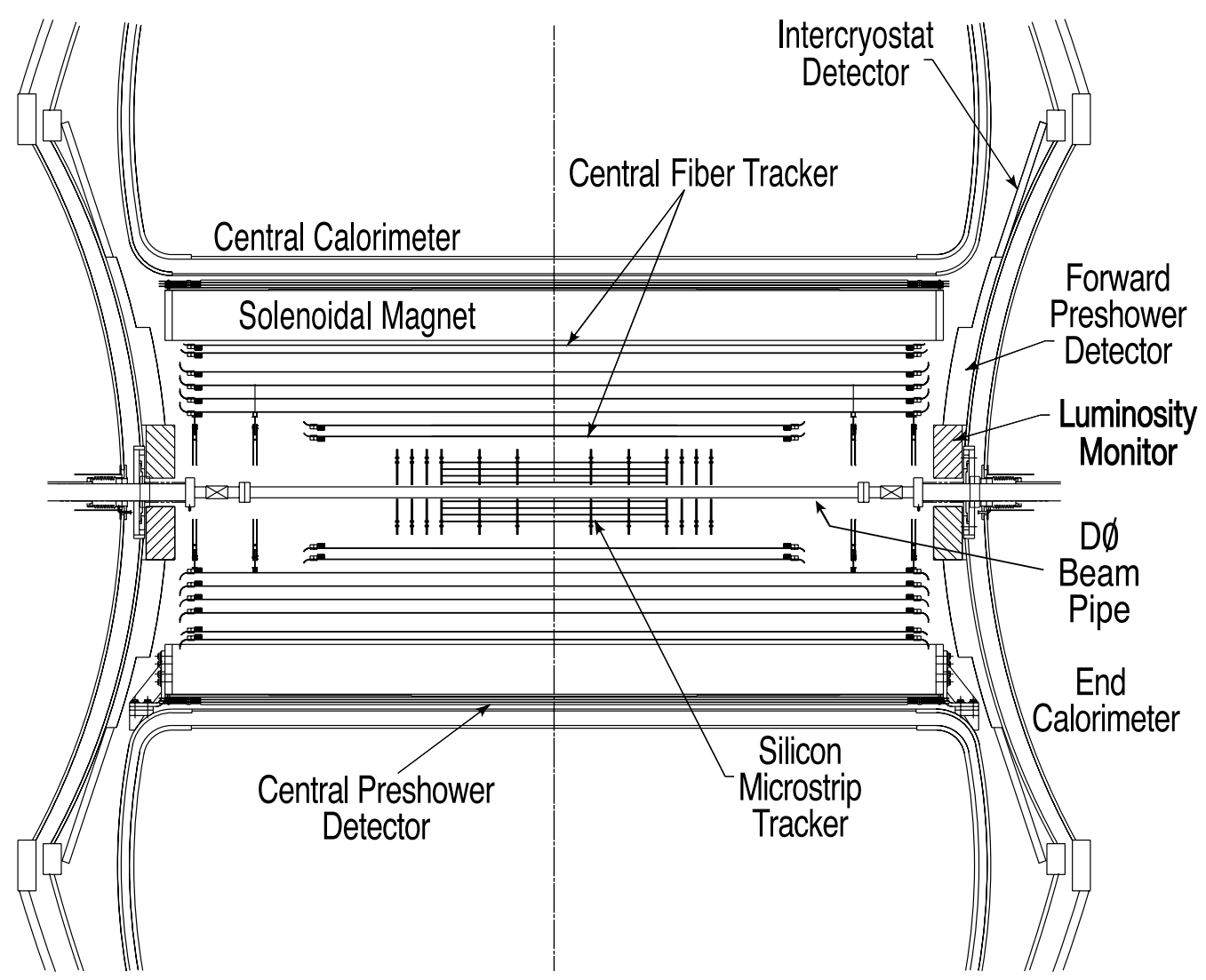

Figure 2.3: The D $\varnothing$ tracking system within the solenoid. The barrels and disks of the silicon microstrip tracker are visible towards the centre, with the layers of fibre tracker arranged around them. The preshower detectors are also seen on the inside surface of the calorimeter [41].

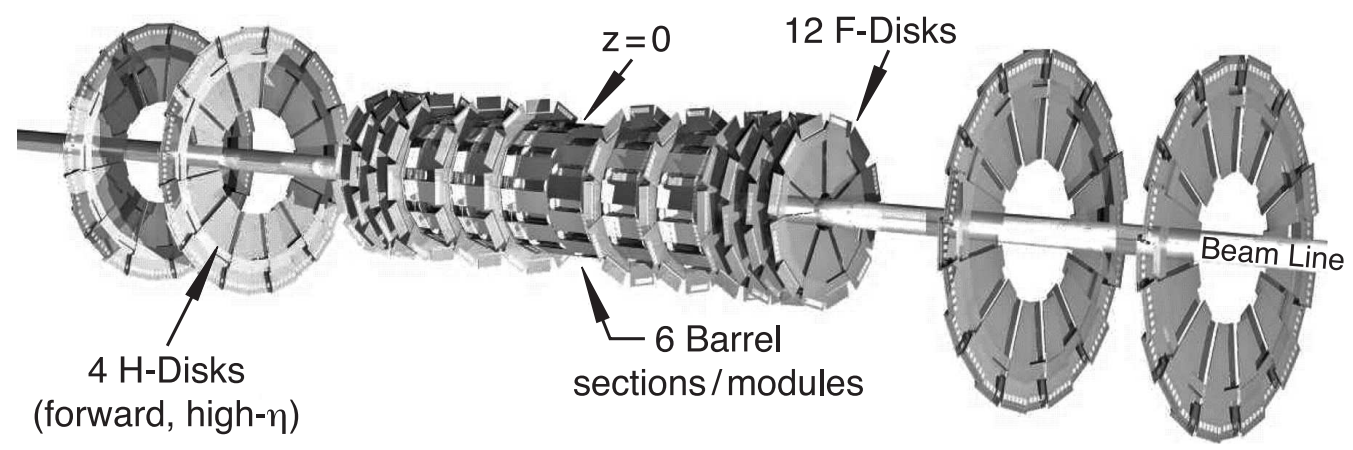

Figure 2.4: The SMT detector. The silicon microstrips are arranged in a number of barrels and disks to enable tracking in the region $|\eta|<3$ [41]. 
- H Disks: The H Disks are located one metre from the centre of the detector, and extend the tracking up to $|\eta|=3.0$. There are four H Disks, two either side of the tracking system. These disks are composed of twelve wedge-shaped, single-sided silicon detectors. The active region extends from a radius of $9.6 \mathrm{~cm}$ to $23.6 \mathrm{~cm}$.

The SMT is able to locate the primary vertex z-position with a resolution of $35 \mu \mathrm{m}$. The tracking system is also able to resolve secondary vertices, with an impact parameter (see Section 2.2.9) resolution of $15 \mu \mathrm{m}$ for particles with $p_{T}$ $>10 \mathrm{GeV}$ within $|\eta|<1$.

\subsubsection{Central Fibre Tracker}

Outside the SMT is the Central Fibre Tracker (CFT); this extends from a radius of $20.04 \mathrm{~cm}$ to $52.15 \mathrm{~cm}$ and provides tracking up to $|\eta|=2.6$. The tracker is made up of scintillating polystyrene fibres arranged in eight cylindrical 'super-layers'. Each super-layer consists of one axial and one stereo layer, at $0^{\circ}$ and $2^{\circ}$ to the z-axis respectively, each comprising 128 fibres. The fibres are offset by half a fibre radius $(417 \mu \mathrm{m})$ so that the fibres overlap with their neighbours..

The fibres are clad in acrylic to guide scintillated photons along the fibre. The scintillating fibres carry this light to polystyrene waveguides, which in turn carry the light to visible light photon counters (VLPCs) which convert the photons into electrical signals with quantum efficiency greater than $75 \%$. The CFT complements the SMT by providing additional tracking, and increases the $p_{T}$ resolution. The combined $p_{T}$ resolution is given (as a percentage) by:

$$
\frac{\delta p_{T}}{p_{T}}=2+0.2 \times p_{T}
$$

where $p_{T}$ is in $\mathrm{GeV}$.

\subsubsection{Solenoid}

An important component of the tracking system is the $2 \mathrm{~T}$ solenoidal magnet which provides a uniform (within 0.5\%) magnetic field for transverse momentum measurements. The solenoid is $2.73 \mathrm{~m}$ long with an external radius of $0.71 \mathrm{~m}$; it is super-conducting, so it is housed in a cryogenic system. The entire solenoid system is 1.1 radiation lengths thick. The polarity of the solenoid can be reversed to reduce systematic uncertainties. 


\subsubsection{Preshower Detectors}

The preshower detectors (PS) are used to measure the point at which particles enter the calorimeter. This helps to match tracks with deposits in the calorimeter, and aids electron identification and background rejection. There are three preshower detectors: one between the solenoid and the central calorimeter and two on the inner faces of the two forward calorimeters. The detectors are made of scintillating tiles that fit the contour of the inner face of the end cryostat. The central preshower (CPS) covers $|\eta|<1.3$ while the forward pre-showers cover $1.5<|\eta|<2.5$ at either end of the detector. The location of the PS is shown in Figures 2.3 and 2.6.

\subsubsection{Calorimeter}

The $\mathrm{D} \varnothing$ experiment measures particle energies with a sampling uranium-liquid argon calorimeter. The incoming particles undergo nuclear collisions in the uranium layers, causing showers of charged particles in the liquid argon. These particles are detected by copper charge collectors, which measure the energy deposited.

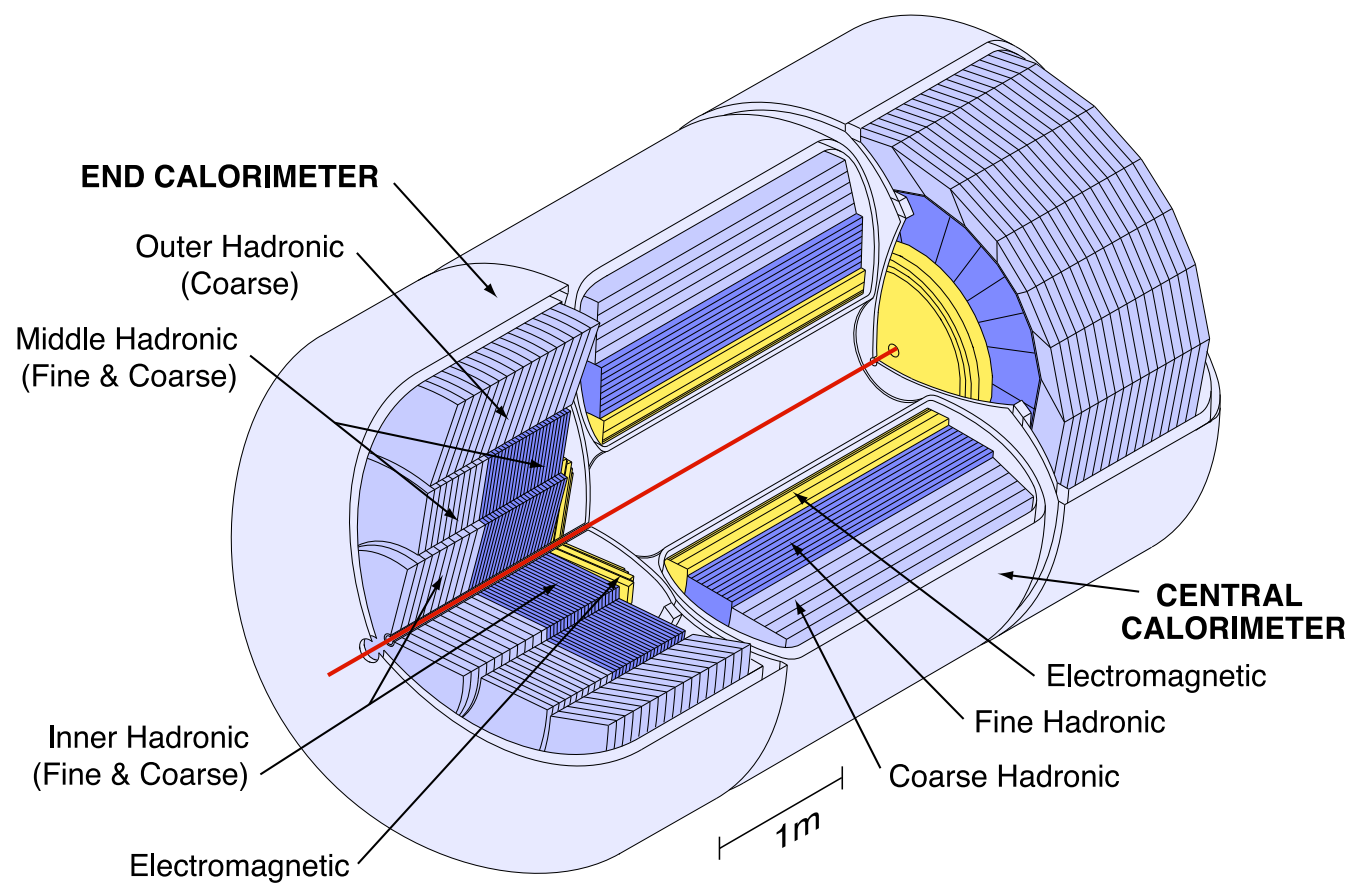

Figure 2.5: Cross section of the $\mathrm{D} \varnothing$ calorimeter, displaying the electromagnetic, fine hadronic and coarse hadronic layers [41].

The calorimeter is cryogenically cooled to keep the argon below its boiling point, and the calorimeter is divided into three cryostats. These sections of the calorimeter are referred to as the central (CC) and north and south end cap (EC) calorimeters. 
These calorimeters contain (moving from the centre of the detector outwards) electromagnetic (EM), fine hadronic and coarse hadronic layers. The arrangement of the layers is shown in Figure 2.5.

The EM section contains four layers of depleted uranium absorber, each 3-4 mm thick, which together provide $20 \chi_{o}$ (electromagnetic radiation lengths) of material in the $\mathrm{CC}$, and $21.4 \chi_{o}$ in the EC. This is enough to prevent most electromagnetic showers from entering the fine hadronic calorimeter. The layers are divided into cells, of size $0.1 \times 0.1(\eta-\phi$ space $)$ in the inner three layers, and $0.05 \times 0.05$ in the fourth layer where the majority of electromagnetic showers are absorbed.

The fine hadronic calorimeter has three $6 \mathrm{~mm}$ layers of depleted uranium (roughly 1 nuclear interaction length, $\lambda$, each), while the coarse hadronic calorimeter has a single layer of copper (CC) or stainless steel (EC) $46.5 \mathrm{~mm}$ thick (providing $4.1 \lambda$ and $4.4 \lambda$ respectively):this layer prevents all but minimum ionising particles from leaving the calorimeter.

The calorimeter response is slightly different for electromagnetic and hadronic particles. The ratio of EM to hadron response was measured in test beam runs, and was found to vary according to energy, from 1.11 at $10 \mathrm{GeV}$ to 1.04 at $150 \mathrm{GeV}$. The energy resolution is also different for EM and hadronic particles:

$$
\begin{gathered}
E M: \frac{\sigma_{E}}{E}=\frac{0.15}{\sqrt{E}}+0.003 \\
\pi^{ \pm}: \frac{\sigma_{E}}{E}=\frac{0.45}{\sqrt{E}}+0.04
\end{gathered}
$$

where energy is in units of $\mathrm{GeV}$.

\subsubsection{Inter-Cryostat Detector}

Because the calorimeter is housed in three cryostats, there is a region between the cryostats (the ICR) without full calorimetry, leading to decreased energy resolution. The inter-cryostat detector is designed to regain some energy resolution by adding extra layers of sampling. The detector is formed of sixteen scintillating tiles on the surface of the forward calorimeters. These tiles have twelve readout sectors of size $0.1 \times 0.1$ in $\eta-\phi$ co-ordinates. The location of the detector is illustrated in Figure 2.3 . 


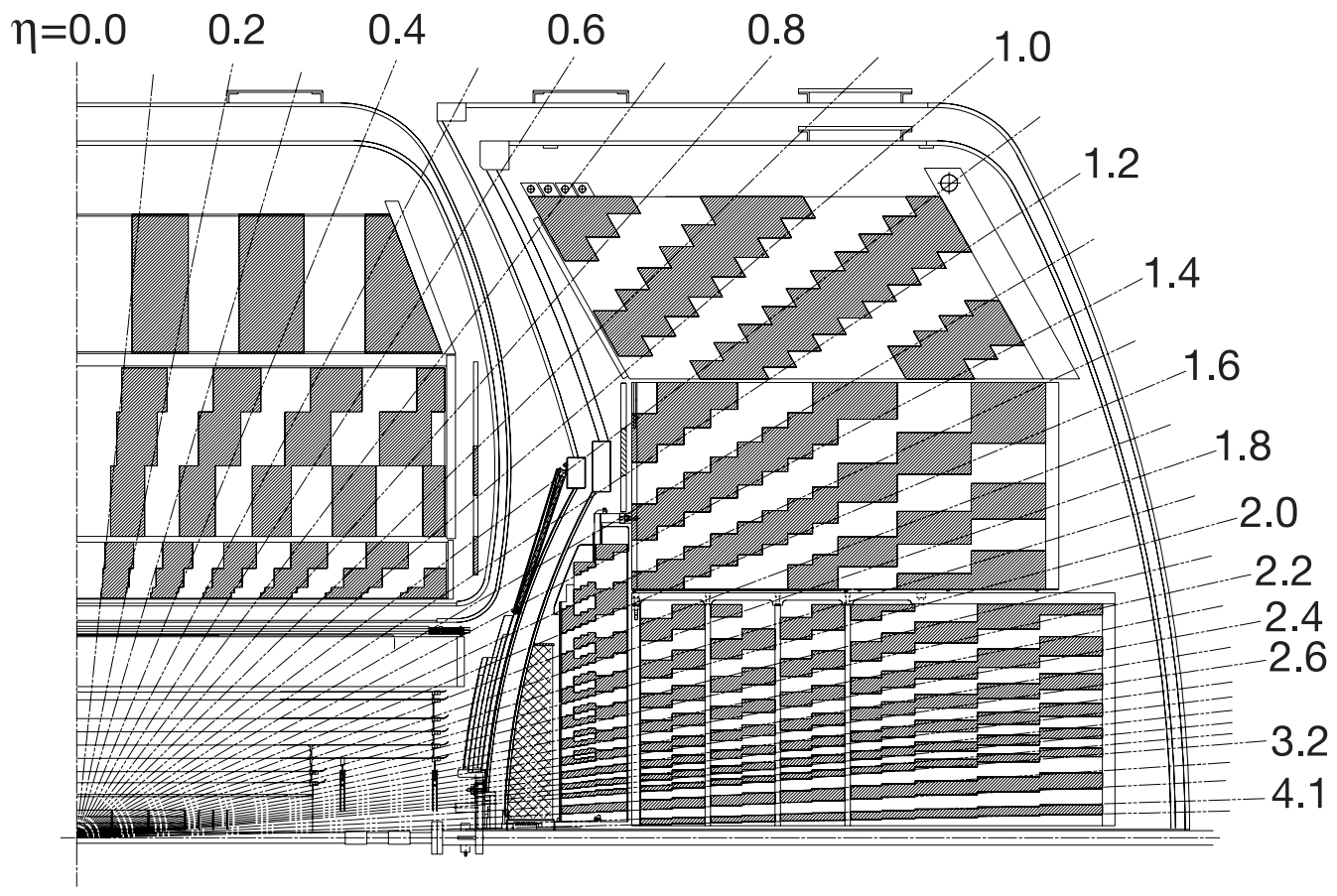

Figure 2.6: Detail of the calorimeter cells, also showing the inter-cryostat region and pre-shower detector [41].

\subsubsection{Muon Detector}

The muon system is located outside of the calorimeter, and uses a series of drift tubes (together with the magnetic field provided by the iron $1.8 \mathrm{~T}$ toroid) to provide muon identification, triggering and momentum measurement. The muon detector is composed of central $(|\eta|<1)$ and forward $(1<|\eta|<2)$ muon systems. Each of these systems contains three layers of drift tubes: Proportional Drift Tubes (PDTs) in the central system, and Mini Drift Tubes (MDTs) in the forward system. The innermost layer, layer $\mathrm{A}$, lies within the toroid, while layers $\mathrm{B}$ and $\mathrm{C}$ are outside the toroid. Layer A contains four sub-layers (decks) of drift tubes, and layers B and $\mathrm{C}$ contain three. The PDTs are divided into $10.1 \mathrm{~cm} \times 10.1 \mathrm{~cm}$ cells and have a maximum drift time of $500 \mathrm{~ns}$. The MDTs are divided into $1 \mathrm{~cm} \times 1 \mathrm{~cm}$ cells and have a maximum drift time of $60 \mathrm{~ns}$. These drift tubes are able to track the muons to provide a secondary measurement of momentum and charge.

In addition to the drift tubes, scintillating counters are included in the muon system: these have a time resolution of $2 \mathrm{~ns}$ and provide a fast muon response. This allows triggering on muons, and provides accurate timing to aid muon tracking. There are two counters in the central muon system (between layer A and the 
calorimeter, and outside layer $\mathrm{C}$ ), and three in the forward muon system (inside layers A and C, and outside layer B).

Muon momenta are measured by both the tracking detector and the muon system; the tracking system measures momentum up to $100 \mathrm{GeV}$, and the resolution is improved by the muon system above this value. The overall momentum resolution is

$$
\frac{0.36(1-p)}{p}+0.03 p \%
$$

where $p$ is in units of $\mathrm{GeV}$.

\subsubsection{Luminosity Monitor}

The luminosity at the $\mathrm{D} \varnothing$ interaction point is measured by the Luminosity Monitor, which counts the average number of inelastic $p \bar{p}$ collisions $\left(\bar{N}_{L M}\right)$. The luminosity can be calculated as follows:

$$
L=\frac{f \bar{N}_{L M}}{\sigma_{L M}}
$$

where $L$ is the luminosity, $f$ is the bunch crossing frequency, and $\sigma_{L M}$ is the cross section of $p \bar{p}$ collisions (taking the efficiency and acceptance of the luminosity monitor into account). The monitor is made up of two arrays of plastic scintillation counters places at $z= \pm 140 \mathrm{~cm}$, just in front of the calorimeter endcaps. The luminosity monitor covers a region $2.7<|\eta|<4$.4.

\subsubsection{Trigger}

The production cross section for minimum bias multijet events from the $p \bar{p}$ collisions is many orders of magnitude greater than the cross section of signal events. Reconstructing, recording and analysing this unwanted data would be prohibitively time consuming and expensive, so a three level trigger is employed to reduce the rate of data from the detector while retaining as many signal events as possible. The event rate and decision time for each trigger level is given in Table 2.2.7.

The $\mathrm{D} \emptyset$ trigger is arranged in three levels: each level uses a basic event reconstruction to determine whether the event meets the conditions to be accepted. The event reconstruction grows more sophisticated as the trigger level increases. The three levels are denoted L1, L2 and L3, and are described in the following sections. 


\subsubsection{Level 1}

The L1 trigger is hardware-based. A set of field programmable gate arrays (FPGAs) uses information from the CFT, PS, calorimeter and muon systems to reject background events, reducing the data rate from $1.7 \mathrm{MHz}$ to $1.6 \mathrm{kHz}$. There are a set of criteria which must be met for each sub-detector. The tracking criteria require tracks to pass a transverse momentum $\left(p_{T}\right)$ threshold; this is achieved by dividing the CFT into $4.5^{\circ}$ sections and comparing the patterns of hits to pre-defined templates for different values of $p_{T}$. The tracks in the CFT are also matched to hits in the PS. Events must also pass energy criteria: the transverse energy $\left(E_{T}\right)$ is summed over all calorimeter layers for $0.2 \times 0.2$ cells in $\eta-\phi$ space; these collections of layers are referred to as 'trigger towers'. Events can then be rejected on the basis of the sum of $E_{T}$ in all the towers, or the number of towers above a threshold $E_{T}$.

The muon trigger matches hits in the muon scintillators and PDTs with tracks in the CFT, and determines whether the pattern of hits is consistent with a muon event. If the event passes a pre-defined set of these criteria, the trigger framework 'fires' the trigger bit, and the event is passed to the L2 trigger.

\subsubsection{Level 2}

The L2 trigger is firmware based (again using FPGAs), and uses information from all sub-detectors used at L1, with the addition of the SMT. At this level, basic reconstruction software forms physics objects (tracks, EM events). This information is passed to a global processor, which combines information from different subdetectors to make physics objects: for example, a track in the CFT may be matched with an EM cluster in the calorimeter to form an electron object. This allows for the overall event to be analysed, and matched with pre-defined trigger signal events.

For each L1 trigger bit that is fired, at least one L2 trigger must be processed. All physics objects required by the trigger are generated and compared with thresholds set in the trigger. If any event meets all conditions in one L2 trigger, it is passed to the L3 trigger.

\begin{tabular}{|c|c|c|}
\hline & Rate & Time \\
\hline$p \bar{p}$ collisions & $1.7 \mathrm{MHz}$ & - \\
L1 & $1.6 \mathrm{kHz}$ & $3.6 \mu \mathrm{s}$ \\
L2 & $800 \mathrm{~Hz}$ & $100 \mu \mathrm{s}$ \\
L3 & $50 \mathrm{~Hz}$ & $150 \mathrm{~ms}$ \\
\hline
\end{tabular}

Table 2.2: Event rate and latency for each trigger level at DØ 


\subsubsection{Level 3}

The L3 trigger is software-based, and runs on a farm of Linux PCs. At this level, events are reconstructed using software similar to the offline (non-trigger) version, but optimised for faster operation.

Having reconstructed the event, one or more L3 triggers are called for each L2 trigger that was fired. The L3 triggers consist of a number of filters; some filters compare physics objects to pre-defined thresholds, while others combine the results of other filters.

If an event passes the trigger it is written to tape, along with a list of triggers it passed. The event is then processed with the offline reconstruction software.

\subsubsection{Offline Reconstruction Software}

Physics events are reconstructed offline in the Fermilab computing farm. There are several versions of the reconstruction software, reflecting the changes in the detector configuration. RunIIa data is reconstructed with code version p17 and RunIIb data with p20. An overview of physics objects used in this thesis, with emphasis on jets, is given below.

- Primary Vertices - Primary vertices are found using an adaptive iterative vertex algorithm [43].

- Calorimeter Jets - Calorimeter jets are reconstructed using the RunII Improved Legacy Cone algorithm [44]. This uses a cone of radius $\Delta R=0.5$ to isolate jets, where $\Delta R=\sqrt{\Delta \eta^{2}+\Delta \phi^{2}}$.

- Track Jets - A track jet is a collection of tracks within $\Delta R<0.5$ of a seed track; these tracks must all have at least two SMT hits. The seed track is required to have $p_{T}>1.0 \mathrm{GeV}$ and there must be more than one track in the cone for a particle to qualify as a track jet.

- Flavoured Jets - In the MC simulations, a jet is known as a b-jet if there is a b-meson within $\Delta R<0.5$. A jet containing a c-meson (but not a b-meson) is denoted a c-jet, while those jets containing only light (uds) quarks are denoted light jets.

- Taggable Jets - Jet identification (tagging) is greatly aided by the ability to detect the vertex of the jet (see section 2.2.9). This requires the jet to satisfy basic tracking criteria, so a jet is considered taggable if the calorimeter jet is matched to a track jet within a cone of $\Delta R<0.5$. 


\subsection{9 b-Jet Identification}

Heavy jets (including b-jets) may be distinguished from light jets by their comparatively long lifetime (of the order of $10^{-12} \mathrm{~s}$ ), and in some cases by the presence of a high $p_{T}$ lepton within the jet (10\% of b-jets decay semi-leptonically). The increased lifetime causes the b-jet to decay some distance from the primary vertex, forming a secondary vertex. This can be reconstructed directly, or measured in terms of impact parameters (IPs). The IP is the distance of closest approach from the track to the primary vertex; the larger this value, the higher the likelihood the track originated from a secondary vertex. The IP and decay length (distance between primary and secondary vertices) are illustrated in Figure 2.7.

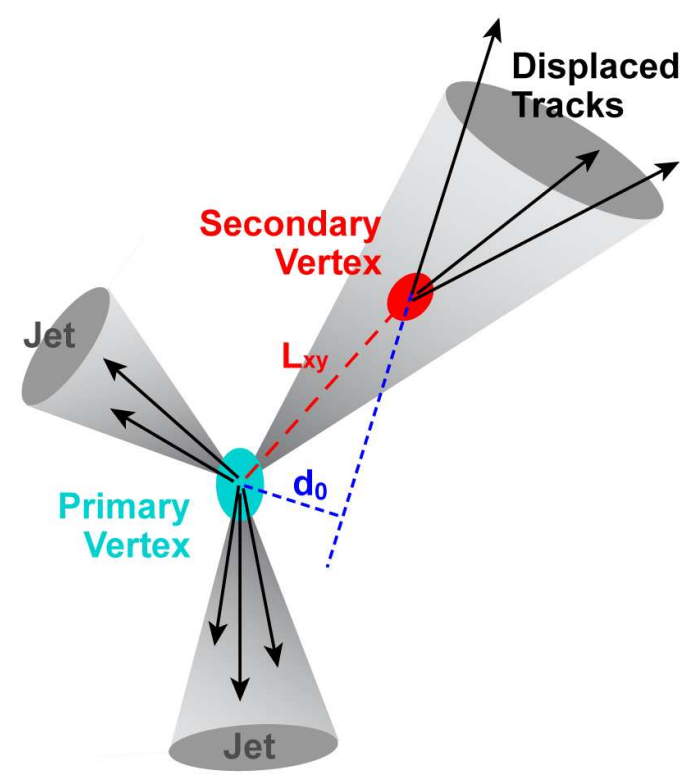

Figure 2.7: An example b decay. The decay length is marked as $L_{x y}$ and the IP is denoted $d_{0}$ [45].

The D $\varnothing$ Collaboration uses several methods to identify (tag) b-jets offline, listed below [46]. The results of these methods are combined using multi-variate analysis (MVA) techniques. The tools that provide inputs to the MVAs are:

- CSIP: The Counting Signed Impact Parameter tagger (CSIP) [47] counts the number of tracks associated with a jet which have an IP significance (the IP divided by its error) greater than a certain threshold. If the jet contains two tracks with IP significance greater than 3 , or three tracks with an IP significance greater than 2 , the jet is tagged. 
- JLIP: The Jet Lifetime Probability Tagger (JLIP) [48] analyses the IPs of all tracks in the b-jet candidate to find the probability that all tracks originate from the primary vertex. Jets are then tagged if this probability is below a threshold value.

- SVT: The Secondary Vertex Tagger (SVT) [49] directly reconstructs the secondary vertex. A jet is tagged if it lies within $\Delta R<0.5$ of a secondary vertex. Additionally, cuts may be placed on the decay length significance of the secondary vertex.

- SLT: The Soft Lepton Tagger (SLT) [50] tags jets if a muon track is located within $\Delta R<0.5$ of the jet.

Currently, two b-tagging algorithms are available: a Neural Network (NN) btagger [51, 52], and the MVA b-tagger [53], which uses a suite of multivariate techniques. The NN b-tagger was the first application of a multivariate analysis technique to jet identification at the Tevatron, and has been in use since 2006. The MVA b-tagger was developed more recently, and includes the NN b-tagger as an input variable. Both taggers have a set of operating points for which the tagging performance is well understood: a comparison of these operating points is given in Table 2.3.

\begin{tabular}{|l|l|l|r|l|r|}
\hline \multicolumn{2}{|c|}{} & \multicolumn{2}{c|}{ NN b-tagger } & \multicolumn{2}{c|}{ MVA b-tagger } \\
\hline Operating Point & Design Fake Rate (\%) & Cut & Eff. (\%) & Cut & Eff. (\%) \\
\hline Tight & 0.3 & $>0.775$ & 48 & $>0.225$ & 54 \\
Medium & 0.5 & $>0.65$ & 54 & $>0.15$ & 59 \\
Loose & 1.0 & $>0.5$ & 59 & $>0.075$ & 62 \\
L2 & 2.0 & $>0.325$ & 65 & $>0.05$ & 68 \\
L3 & 3.0 & $>0.25$ & 69 & $>0.042$ & 72 \\
L4 & 4.0 & $>0.2$ & 71 & $>0.035$ & 75 \\
\hline
\end{tabular}

Table 2.3: The fake rates and efficiencies for various operating points of the NN and MVA btaggers. The range of outputs for both b-taggers is from 0 (less b-jet like) to 1 (more b-jet like).

\subsubsection{1 b-Tagging in the L3 Trigger}

In addition to the offline b-tagging, some b-tagging takes place in the L3 trigger. This process is much less sophisticated than the offline version because the decision time is limited to $150 \mathrm{~ms}$ on average. There are currently two b-tagging tools at L3: 
- L3 IP: The Level 3 Impact Parameter (L3 IP) [54] tagger calculates the IP significance of each track in a jet, and from this the probability that the jet originated from the primary vertex. The probabilities for each jet are combined into an event probability: this value is close to 0 if the event is likely to contain a b-jet.

- L3 SVT: The Level 3 Secondary Vertex Tagger [55] reconstructs secondary vertices directly, employing a fast IP minimisation technique in order to reduce computation time. The IP significance to the primary vertex and to a number of evaluation points (EPs) is calculated for all tracks. Tracks with a high IP significance with respect to the primary vertex and a low IP significance with respect to the EP are investigated for secondary vertices by the tool.

The L3 SVT tagger was added recently to complement the L3 IP tagger. The IP tagger detects whether an event is likely to contain b-jets, while the SVT tagger gives further information on any secondary vertices. These tools allow the identification of b-jet events at the trigger level with high efficiency: this ability is important for the analysis described in Chapters 3 to 5 .

As part of the work for this thesis, the SVT tagger performance was analysed, and the author contributed to its commissioning. The SVT tool calculates the decay length, decay length significance and $\chi^{2}$ for each track, and also returns the number of vertices in the event. Any of these values may be used to set criteria for an event to pass the trigger, but currently only the decay length significance is used (the threshold is set to 3.0).

When assessing the triggers, the trigger rate and efficiency must be taken into account. The efficiency is measured using a b-enhanced sample; the efficiency is given by the number of events that pass a certain threshold divided by the total number of events. The trigger rate is the rate at which the trigger is fired during detector operations. This rate can be predicted using the Trigger Rate Tool (TRT). The TRT is a fast simulation of the trigger, and provides a good approximation of the actual rate. The author was responsible for including the SVT trigger in the TRT. The TRT predicted that the SVT trigger would fire at a rate of $2.24 \pm 0.05$ $\mathrm{Hz}$, compared with $2.28 \mathrm{~Hz}$ in data at a comparable instantaneous luminosity (the exclusive rate was much lower than this, because most events that fired the SVT trigger also fired the IP trigger). This allowed the effect of different combinations of the b-tagging triggers to be evaluated before adding them to the trigger list. The SVT trigger performance for various decay length significance cuts is shown in Figure 2.8 [57], as determined by the author. Plots of this type were taken into account when optimising the performance of the SVT trigger, both individually and in conjunction with other triggers. 


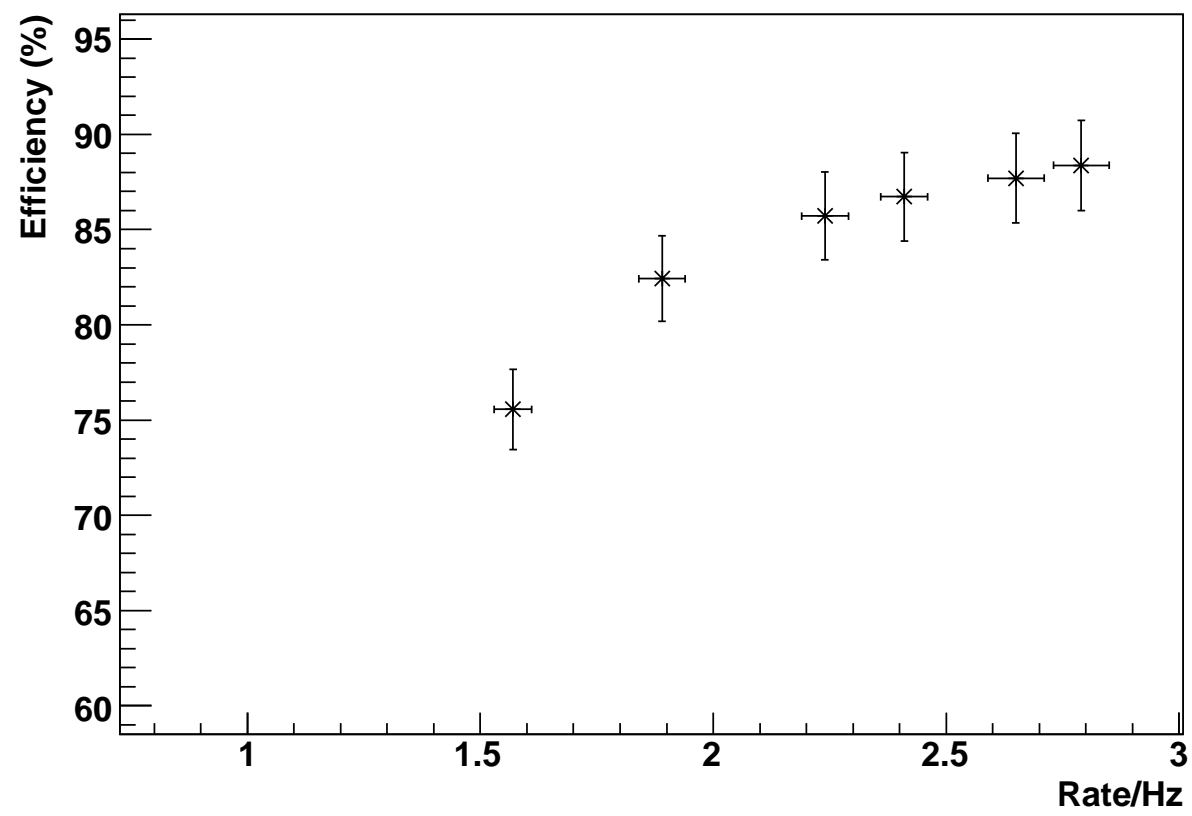

Figure 2.8: The trigger efficiency with respect to offline reconstruction, plotted as a function of trigger rate for the SVT trigger. The points represent decay length significance cuts of $8,6,3,2$, 1 and 0 from left to right. [57]

\subsubsection{Detector Performance}

The $\mathrm{D} \varnothing$ detector continues to run well, collecting data with an efficiency of over 90\%, as shown in Figure 2.9. The detector has experienced some degradation since the start of Run II, but this is minimal. For example, $99 \%$ of all muon drift tubes and $99 \%$ of calorimeter channels are operational [58]. 99\% of calorimeter channels are also operational.

During RunII the tracker occupancy increased: the effect of increased luminosity on the L3 tracking algorithms have been studied as part of this $\mathrm{PhD}$. It was found that the L3 tracking efficiency decreased linearly as the instantaneous luminosity increased. This was expected, as the increasing luminosity leads to greater numbers of particles being emitted at each bunch crossing. These particles are detected as a series of hits (points where particles interact with the tracking sensors) in the tracking system. As the number of hits increases, the more difficult it becomes to construct individual tracks. This has a large impact on the tracking triggers, where reconstruction must be completed within a time limit. However, this reduction in efficiency was small in comparison with the increase in the number of tracks due to the high luminosity delivered to the detector, so the overall effect on detector performance was minimal. 


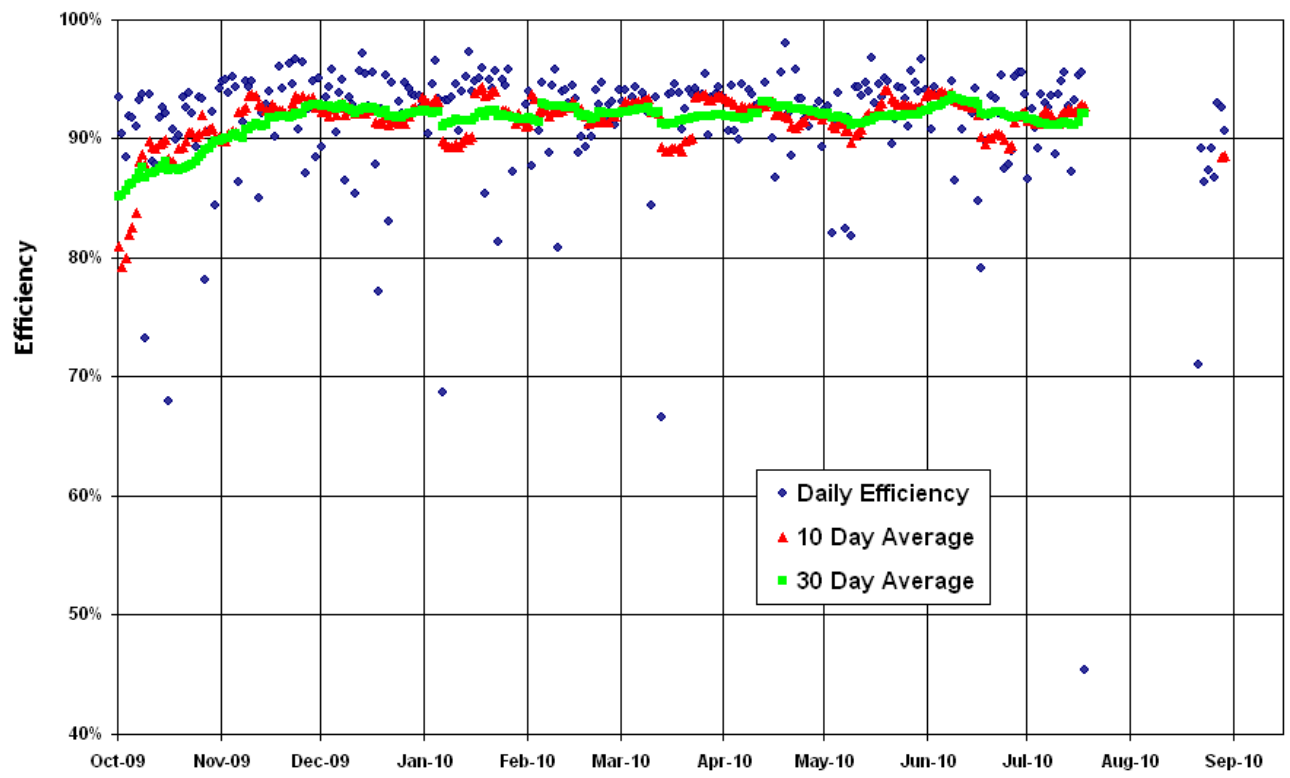

Figure 2.9: The $\mathrm{D} \varnothing$ daily data taking efficiency for the period October 2009 to September 2010. The data taking efficiency is the ratio of recorded to delivered data. The efficiency is generally above $90 \%$, allowing a large amount of data to be collected and analysed [59].

Overall the experiment continues to perform well, allowing a wide range of physics analyses to be carried out. 


\section{Chapter 3}

\section{Search for Neutral \\ Supersymmetric Higgs Bosons in the Multijet Channel}

\subsection{Introduction}

Chapters 3, 4 and 5 describe a search for neutral MSSM Higgs bosons $(\phi)$ in the multijet channel, using $6.6 \mathrm{fb}^{-1}$ of data from RunII of the DØ detector. Both the SM and MSSM predict a large branching ratio for low mass Higgs boson decays to b-quark pairs, which makes a search for multiple b-jet Higgs boson events viable in spite of the large multijet background inherent at a hadron collider: this is described in Section 3.1.1. The author was responsible for a significant change in analysis method from the previous analysis [60]: an overview of the previous method is given in 3.1.2, and the new method is presented in the following sections. Details of the data samples, Monte Carlo simulations, event selection and background prediction are given in Section 3.2. Section 4.1 gives an overview and comparison of different multivariate techniques that were considered for the analysis. The systematic uncertainties are listed in Section 5.2, and expected confidence limits in the $m_{A}, \tan \beta$ plane are given in Section 5.3.

\subsection{1 $\phi \mathbf{b}(\mathbf{b}) \rightarrow b \bar{b} b(b)$ in the MSSM}

The branching ratios of various Higgs decay processes in the SM are given in Figure 3.1 (a). The Higgs boson decay to b-quark pairs dominates for Higgs boson masses below $135 \mathrm{GeV}$, above which point diboson decays are more prevalent. In the MSSM however, the $A$ boson does not decay to $W$ boson pairs, so the process $A \rightarrow b \bar{b}$ is the 
dominant decay over a larger mass range, as seen in Figure 3.1 (b). The branching ratio of $H$ and $h$ bosons to $W$ bosons is suppressed at high $\tan \beta$, so the neutral Higgs bosons (collectively labelled $\phi$ ) decay predominantly to $b \bar{b}$. Approximately $90 \%$ of neutral Higgs boson decays are to b-quark pairs, with the other $10 \%$ mainly comprising $\tau^{+} \tau^{-}$decays.

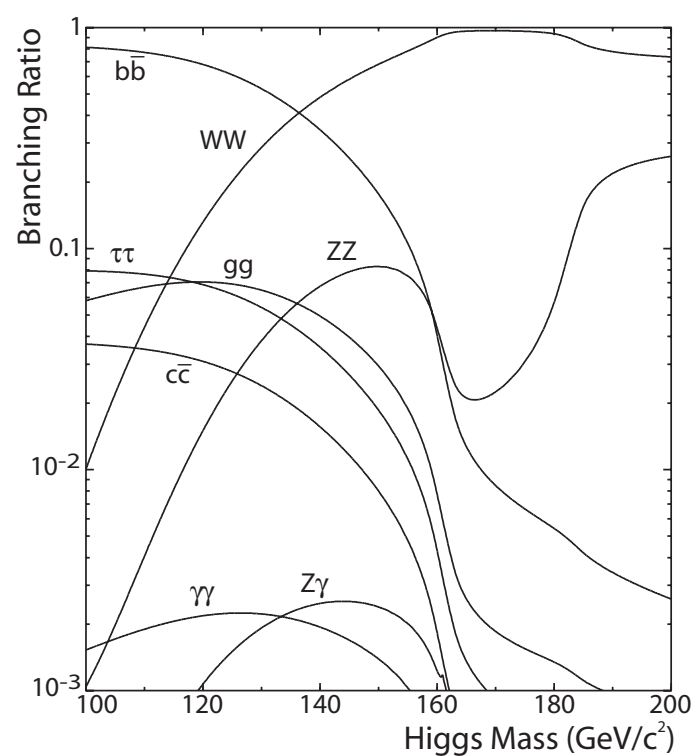

(a)

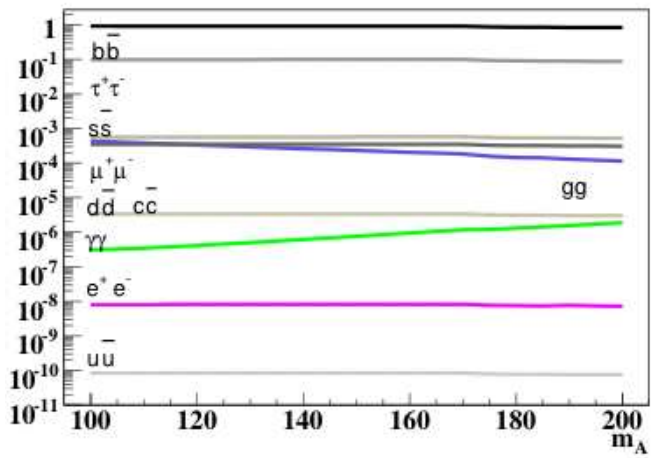

(b)

Figure 3.1: Higgs boson branching ratios as a function of Higgs mass for the (a) the Standard Model and (b) the MSSM [61]. The MSSM graph gives branching ratios for the $A$ boson; the $A$ boson coupling to up type quarks is suppressed, and the $A$ boson does not couple to the weak bosons.

The channel $\phi \rightarrow b \bar{b}$ would be extremely difficult to distinguish from the large number of multijet events. A common strategy to reduce the proportion of background b-jet pair events in the data sample is to require the presence of a spectator particle produced in association with the Higgs boson. In the MSSM, the cross section of the process $b \rightarrow \phi b$ has a $\tan ^{2} \beta$ enhancement for two of the three neutral Higgs bosons, making this a suitable associated production channel for searches. Feynman diagrams for the three- and four-jet versions of this process are given in Figure 3.2.

Overall, the MSSM cross section multiplied by branching ratio $(\sigma \times B R)$ for this process can be expressed as:

$$
(\sigma \times B R)_{M S S M} \approx \sigma_{S M} \times 2 \times 0.9 \times \tan ^{2} \beta
$$

where $\sigma_{S M}$ is the cross section of the SM Higgs boson [62]. 

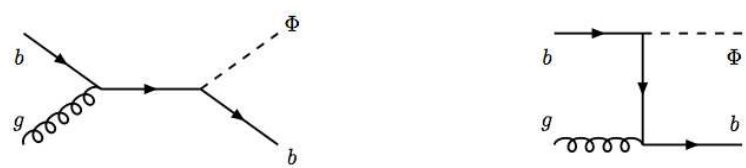

(a)
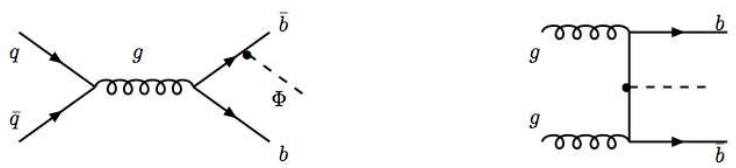

(b)

Figure 3.2: Feynman diagrams for (a) three and (b) four jet $\phi b(b) \rightarrow b \bar{b} b(b)$ decays. The process $b \rightarrow \phi b$ is enhanced by a factor of $\tan ^{2} \beta$ in the MSSM.

The search described in this thesis was essentially a model independent search: the enhancement in Equation (3.1) was used to convert the model independent cross section limits to naive limits on $\tan \beta$ in the MSSM, ignoring SUSY loop corrections to the value of $\tan \beta$ and the effect of the increased Higgs boson coupling on the width. These effects are further discussed in Section 5.3.2.

A brief summary of previous searches in this channel at $\mathrm{D} \varnothing$ is given in the next Section.

\subsubsection{Overview of Previous Analysis Versions}

The published result in this channel is the $1 \mathrm{fb}^{-1}$ analysis $[1,66]$. An updated publication using $5.2 \mathrm{fb}^{-1}$ of data and the same analysis procedure as the published result is currently in the final stages of review [60]. In both cases, a sample of events with three or four jets, at least three of which were identified as b-jets by the DØ neural network b-tagger, was analysed. The b-tagging requirement removed a high proportion of the light jet events, although light jets mis-identified as heavy jets remained in the sample. Most $b \bar{b}$ events at $\mathrm{D} \varnothing$ are due to QCD processes, so these dominated the b-tagged sample. The sensitivity of these searches relied on accurate modelling of the multijet background. The relative contributions and cross sections of the various background processes could not be predicted theoretically, so a data-driven background model was employed.

Each event in the sample contained at least three b-tagged jets: in signal events, two of these jets would result from the decay of the Higgs boson. Thus, each pairing (from the leading three jets) was considered to be a potential Higgs candidate. The likelihood that each jet pair was derived from a Higgs boson was calculated, and the pair with the highest likelihood was retained. The likelihood was calculated using a number of kinematic variables for each jet pair. The di-jet invariant mass was not 
included in this calculation, so the likelihood was not sensitive to any one value of $m_{A}$. Separate high and low Higgs boson mass likelihoods were used to optimise the analysis for these regimes. An additional cut was placed on the likelihood to remove background-like events

The final variable used to set confidence limits on the Higgs boson cross section (and so the $m_{A}, \tan \beta$ plane) was the di-jet invariant mass of the selected jet pair from each event. The limits on $\sigma \times B R$ calculated for $1 \mathrm{fb}^{-1}$ of data are given in Figure 3.3.

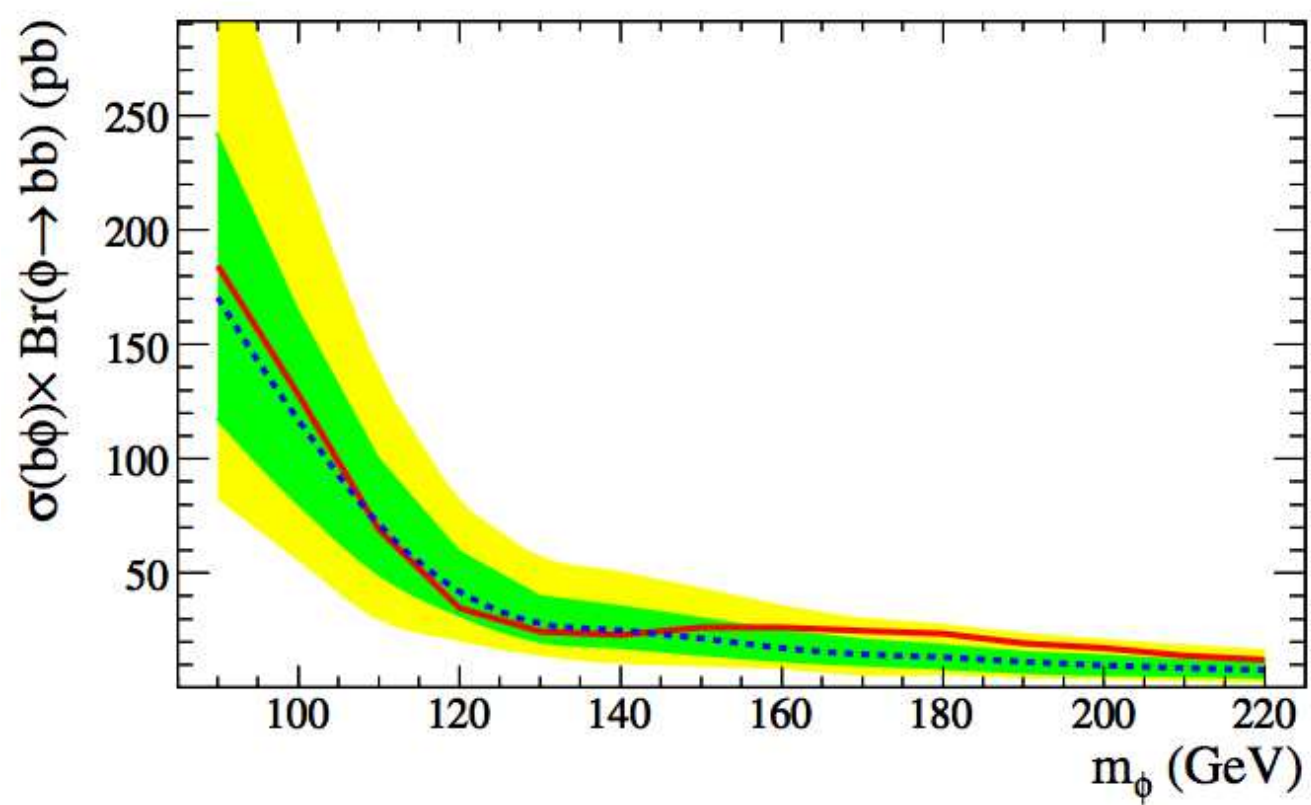

Figure 3.3: Median expected (dotted blue line) and observed (solid red line) limits on $\sigma \times B R$ for the $\phi b \rightarrow b \bar{b} b$ process as a function of Higgs boson mass, using $1 \mathrm{fb}^{-1}$ of DØ RunII data [66]. The green and yellow bands correspond to the expected limit where the expected number of events is varied within $\pm 1 \sigma$ and $\pm 2 \sigma$ respectively.

The core of the analysis was largely unchanged for the version presented in this thesis. The method for predicting the background was kept, but in a modified form. An extra $1.4 \mathrm{fb}^{-1}$ of data were analysed, the MVA b-tagger was used in place of the NN b-tagger, and the pair-based selection was replaced with a method that took the event as a whole into account. A more powerful multivariate approach was used to provide the final discriminant.

\subsection{Analysis Method}

A large proportion of Tevatron collisions result in multiple light jet events, some of which have b-jet like properties. As the signature for this channel involves multiple 
b-jets, the multijet background must be understood. Monte Carlo simulation is not able to reliably predict the shape or scale of this background, so a data-based background prediction was used. The methods used to study the background, identify signal events and evaluate confidence limits are described in this section.

\subsubsection{Data Sample}

The set of data events containing three or more jets [64] (the three jet skim) was used for this analysis. This skim contains events with one jet with transverse momentum $\left(p_{T}\right)$ greater than $20 \mathrm{GeV}$, and two others with $p_{T}$ greater than $15 \mathrm{GeV}$; these jets were required to have a pseudorapidity $(\eta)$ within \pm 2.5 . Events were taken from runs where no detector problems or errors in the luminosity calculation were reported. These data quality cuts removed approximately $13 \%$ of the events: of the remaining events, $6 \%$ were affected by known calorimeter issues, so these were also removed. This left roughly 351 million events to analyse.

\subsubsection{Trigger}

The data were collected using a dedicated $\phi b \bar{b}$ trigger. The RunIIa data were collected using trigger list versions 8 to 14; the triggers used for this analysis are described in Table 3.1.

Two trigger list versions, v15 and v16 [67], were used for RunIIb. In v15, the analysis was run over events that fired the joint impact parameter (IP) and secondary vertex (SVT) triggers, and a silicon track trigger (STT) impact parameter requirement was added to this trigger at Level 2 in v16. A modified form of this trigger was added in the v16 trigger list: this trigger did not have the STT impact parameter requirement at Level 2, nor any b-tagging requirement at Level 3 (L3BTAG). The frequency at which this non b-tagging trigger was fired was restricted by turning the trigger off at instantaneous luminosities of $3 \times 10^{32} \mathrm{~cm}^{-2} \mathrm{~s}^{-1}$ and higher, and pre-scaling the trigger at luminosities between $3 \times 10^{32} \mathrm{~cm}^{-2} \mathrm{~s}^{-1}$ and $0.6 \times 10^{32} \mathrm{~cm}^{-2} \mathrm{~s}^{-1}$. The RunIIb triggers are listed in Table 3.2.

Any changes between trigger list versions were accounted for in the MC simulation, so it was not necessary to analyse data from different trigger versions separately unless the detector configuration (RunIIa or RunIIb) was different.

\subsubsection{Jets}

The DØ RunII Improved Legacy Cone algorithm [44, 68] was used to reconstruct the jets. The jet energy and jet energy resolution were corrected using the DØ Jet 


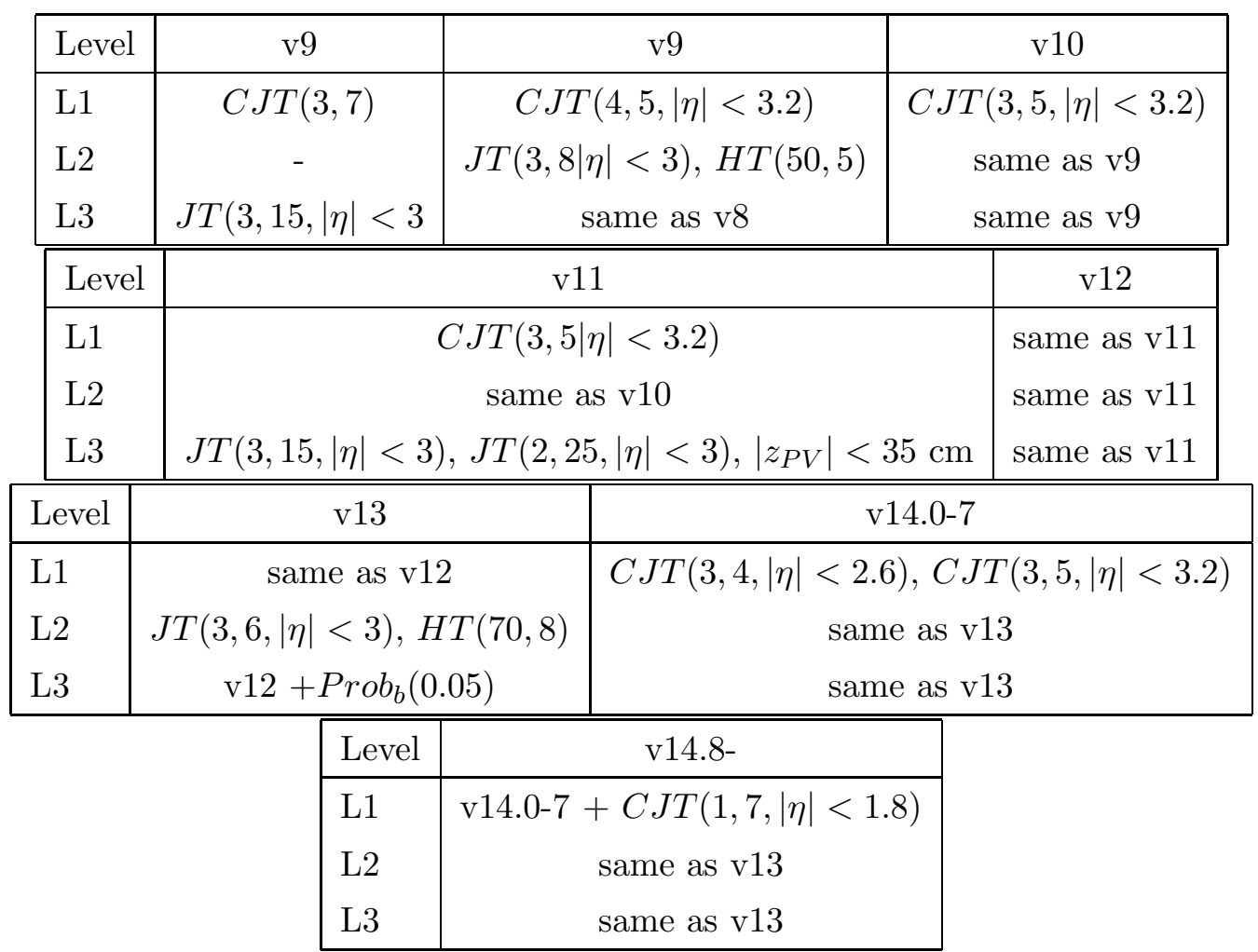

Table 3.1: List of triggers used in the p17 (RunIIa) analysis. $C J T(x, y,|\eta|<z)$ corresponds to a requirement of $x$ calorimeter trigger towers with energy greater than $y \mathrm{GeV}$ within $|\eta|<z$. The $J T(x, y,|\eta|<z)$ term indicates a requirement of $x$ jets with $p_{T}>y \mathrm{GeV}$ and $|\eta|<z$. The L2 $H T(x, y)$ term requires that the sum of the transverse energy of jets with $p_{T}>y \mathrm{GeV}$ is greater than $x \mathrm{GeV}$. The $z_{P V}$ term is a requirement on the distance of the primary vertex from the centre of the detector (measured along the beam axis), and the $\operatorname{Prob}_{b}(x)$ term indicates that the output of the L3 IP tagger must be less than $x$ for the event to fire the trigger.

Energy Scale (JES [69]) and Jet Shifting, Smearing and Removal (JSSR [70]) tools (these applied energy corrections derived from studies of photon-jet events in data). The energies of jets containing a muon were also corrected to compensate for the energy carried by the muon and neutrino.

\subsubsection{Monte Carlo Samples}

A number of multijet background samples were simulated using ALPGEN v2.11 [71]: these are listed in Table 3.3. The cross sections for the background samples were predicted to leading order. Although these samples formed part of the process of modelling the background, they were not used as the background prediction itself: see section 3.2.6 for more details.

As the main difference between the MSSM $\phi b \rightarrow b \bar{b} b$ process and its SM counterpart is the value of $\sigma \times B R$ (to leading order), signal MC samples were generated 


\begin{tabular}{|l|c|}
\hline Level & $\mathrm{v} 15$ \\
\hline L1 & $\operatorname{CSWJT}(3,8,|\eta|<3.2) \operatorname{CSWJT}(2,15,|\eta|<2.4) \operatorname{CSWJT}(1,30,|\eta|<2.4)$ \\
L2 & $\mathrm{JT}(3,8,|\eta|<\mathrm{X}) \mathrm{HT}(75,6) \operatorname{SPHER}(0.1) \mathrm{OR}$ \\
& $\mathrm{JT}(1,30,|\eta|<2.6) \mathrm{JT}(2,15,|\eta|<2.6) \mathrm{JT}(3,8,|\eta|<\mathrm{X}) \mathrm{HT}(75,6) \mathrm{MJT}(10,10) \mathrm{OR}$ \\
& $\mathrm{JT}(1,30,|\eta|<2.6) \mathrm{JT}(2,15,|\eta|<2.6) \mathrm{JT}(3,8,|\eta|<\mathrm{X}) \mathrm{HT}(100,6)$ \\
$\mathrm{L} 3$ & $\mathrm{JT}(3,15,|\eta|<3.6) \mathrm{JT}(2,25,|\eta|<3.6)|\mathrm{zPV}|<35 \mathrm{~cm} \mathrm{BTAG}(0.4)$ \\
\hline \hline Level & $\mathrm{v} 16$ \\
\hline L1 & $\mathrm{CSWJT}(3,8,|\eta|<3.2) \operatorname{CSWJT}(2,15,|\eta|<2.4) \operatorname{CSWJT}(1,30,|\eta|<2.4)$ \\
L2 & $\mathrm{JT}(1,30,|\eta|<2.6) \mathrm{JT}(2,15,|\eta|<2.6) \mathrm{JT}(3,8,|\eta|<\mathrm{X}) \operatorname{HT}(75,6) \mathrm{MJT}(20,10) \mathrm{OR}$ \\
& $\mathrm{JT}(1,30,|\eta|<2.4) \mathrm{JT}(2,15,|\eta|<2.4) \mathrm{JT}(3,8,|\eta|<2.4) \operatorname{HT}(75,6) \operatorname{STTIP}(1,5.5,3)$ \\
L3 & $\mathrm{JT}(3,15,|\eta|<3.6) \mathrm{JT}(2,25,|\eta|<3.6)|\mathrm{zPV}|<35 \mathrm{~cm} \mathrm{BTAG(0.4)}$ \\
\hline
\end{tabular}

Table 3.2: The $\phi b \bar{b}$ trigger conditions in the $\mathrm{v} 15$ and $\mathrm{v} 16$ trigger lists. The following requirements are made: the CSWJT $(\mathrm{x}, \mathrm{y},|\eta|<\mathrm{z})$ term requires $\mathrm{x} \mathrm{L1}$ jets with energy greater than $\mathrm{y} \mathrm{GeV}$ and with $|\eta|<\mathrm{z}$. The $\mathrm{JT}(\mathrm{x}, \mathrm{y},|\eta|<\mathrm{z})$ term requires $\mathrm{x}$ L2 or L3 jets with $p_{T}$ greater than $\mathrm{y}$ and $\mathrm{z}$ within $|\eta|<\mathrm{z}$. The L2 HT(x,y)term requires that the sum of the transverse energies of jets with $p_{T}>\mathrm{y}$ $\mathrm{GeV}$ is greater than $\mathrm{x} \mathrm{GeV}$, while the $\operatorname{SPHER}(\mathrm{x})$ term requires the event sphericity measured at L2 is greater than $\mathrm{x}$. The MJT(x,y) term is similar to the HT term in that it requires the total missing transverse energy (MET) of jets with transverse energy $>\mathrm{y} \mathrm{GeV}$ to be greater than $\mathrm{x} G \mathrm{GeV}$. The STTIP $(\mathrm{x}, \mathrm{y}, \mathrm{z})$ term requires $\mathrm{x}$ L2 STT tracks with impact parameter significance greater than or equal to $\mathrm{z}$, with a $\chi^{2}<\mathrm{y}$. $\left|z_{P V}\right|<35 \mathrm{~cm}$ is a requirement that the primary vertex reconstructed at L3 is within $35 \mathrm{~cm}$ of the centre of the detector, measured along the beam axis. The BTAG(0.4) term requires the L3 IP tagger to return a value less than 0.4.

using a SM simulation. Signal samples were generated for mass hypotheses from $90 \mathrm{GeV}$ to $320 \mathrm{GeV}$, in $10 \mathrm{GeV}$ increments. The sample cross sections were set to leading order (LO) using Pythia v6.409 [72], with next to leading order corrections calculated using MCFM [73] (using the CTEQ6 parton density functions [74]). The MC signal samples are listed in Table 3.4.

\subsection{3 b-Tagging}

A description of the different b-tagging algorithms is given in Chapter 2. The multivariate analysis (MVA) b-tagger has shown higher efficiency than the neural network b-tagger in tests [63], and was used to analyse RunIIb data and MC. The MVA b-tagger was not available in p17 at the time, so the NN b-tagger was used to analyse the RunIIa data and MC.

To be included in the analysis, data events had to contain three tight-tagged jets (as defined in Table 2.3) according to the relevant b-tagger (NN for RunIIa, MVA for RunIIb). In the MC, however, the base MC event weights were multiplied by 


\begin{tabular}{|l|c|c|}
\hline Sample & Number of Events (p17) & Number of Events (p20) \\
\hline bbb & $3,076,000$ & $4,600,000$ \\
bbc & $1,846,000$ & $3,648,000$ \\
bbj & $1,632,500$ & $3,000,000$ \\
bbjj & $1,880,750$ & $3,000,000$ \\
ccj & 816,000 & $2,000,000$ \\
ccjj & 806,000 & $2,000,000$ \\
tt & 421,068 & 359,830 \\
\hline
\end{tabular}

Table 3.3: Number of events in each MC background sample.

scale factors, derived by the b-ID group, which corresponded to the probability that event would be selected in a three tight-tagged sample by the relevant b-tagger. A taggability scale factor was applied: the taggability is defined as the number of good, taggable jets divided by the total number of jets. This value varies as a function of $\eta$ and $p_{T}$ : these functions are measured in data and $\mathrm{MC}$, and the $\mathrm{MC}$ taggability functions are corrected to data [75]. The corrected taggability functions were used to scale $\mathrm{MC}$ events.

The MC event weights were multiplied by the event b-tagging efficiencies. These vary as a function of $p_{T}$ and $\eta$, and are referred to as Tag Rate Functions (TRFs) [52] (again, these are corrected to data). The analysis described in this thesis used the TRFs to model b-tagger response in the MC events.

\subsubsection{Event Selection}

In order to be included in the analysis, events were required to:

- Fire the $\phi b \bar{b}$ trigger

- Have the primary vertex within $|z|<35 \mathrm{~cm}$

- Contain 2 tight-tagged jets with $p_{T}>25 \mathrm{GeV}$ and $|\eta|<2.5$

- Contain 1 or 2 additional tight-tagged jets with $p_{T}>20 \mathrm{GeV}$ and $|\eta|<2.5$

- Contain at least one pairing of these jets with invariant mass $>50 \mathrm{GeV}$

- Have $\Delta R>1.2$ for the second and third leading jets. 


\begin{tabular}{|l|c|c|}
\hline$m_{h} / \mathrm{GeV}$ & Number of Events (p17) & Number of Events $(\mathrm{p} 20)$ \\
\hline 90 & 299,500 & 600,000 \\
100 & 300,000 & 600,000 \\
110 & 300,000 & 600,000 \\
120 & 300,000 & 600,000 \\
130 & 301,000 & 600,000 \\
140 & 305,500 & 600,000 \\
150 & 307,500 & 600,000 \\
160 & 300,000 & 600,000 \\
170 & 300,000 & 600,000 \\
180 & 315,500 & 600,000 \\
190 & 299,750 & 600,000 \\
200 & 331,500 & 600,000 \\
210 & 298,500 & 600,000 \\
220 & 359,500 & 600,000 \\
230 & 300,000 & 300,000 \\
240 & 299,000 & 300,000 \\
250 & 300,000 & 300,000 \\
260 & 600,000 & 300,000 \\
270 & 300,000 & 100,250 \\
280 & 300,000 & 100,084 \\
290 & 300,000 & 100,000 \\
300 & 298,000 & 1000 \\
310 & 300,000 & \\
320 & 300,000 & \\
\hline
\end{tabular}

Table 3.4: The number of events in the p17 and p20 MC signal samples. 
The MC simulation did not include jet pairs from gluon splitting, so these had to be removed from data. This could be achieved by removing events where one pair out of the leading three jets had a low value of $\Delta R$, but it was found that cuts on the $\Delta R$ of pairs containing the leading $p_{T}$ jet had little effect on the data-MC agreement. In four-jet events, imposing cuts on the $\Delta R$ of pairs including the fourth jet in $p_{T}$ order had negligible effects on the number of events in data, so the cut $\Delta R>1.2$ cut was imposed on the pair containing the second- and third-leading jets (this improved the data-MC agreement in other kinematic variables).

The number of data events passing these cuts are shown in Tables 3.5 and 3.6. To reduce the time taken to process data, only events with more than one tight btagged jet were analysed: this selection places a cut on the primary vertex position, so the primary vertex cut is not included in the Tables. In addition, only events with three or four jets were processed for the RunIIb data, again to reduce processing time.

\begin{tabular}{|l|c|c|}
\hline & Number of events & $\begin{array}{c}\text { Fraction relative } \\
\text { to previous level }\end{array}$ \\
\hline Events with $>1$ NN tight $b$-tag jet & 438,549 & - \\
Fire $\phi b \bar{b}$ trigger & 289,271 & 0.660 \\
$>2$ good, taggable jets & 243,013 & 0.840 \\
$>2$ NN tight $b$-tag jets & 6,386 & 0.026 \\
$\Delta R_{\text {(jet } 2 \text {, jet 3) }>1.2}$ & 5,013 & 0.784 \\
with 3 jets (exclusive) & 2,414 & 0.482 \\
with 4 jets (exclusive) & 2,014 & 0.401 \\
\hline
\end{tabular}

Table 3.5: The number of events and relative fraction of events in data passing each cut in RunIIa.

\subsubsection{Background Composition}

The fractional contributions of the event types generated in $\mathrm{MC}$ were calculated by comparing the total jet transverse energy $\left(H_{T}\right)$ distributions for the MC samples with those for data in the three- and four-jet channels with different b-jet tagging cuts applied. The three jet channel has nine possible combinations of light $(j)$, charm $(c)$ and bottom (b) jets: $j j j, c j j, b j j, c c j, b b j, c c c, b c c, b b c$, and $b b b(b c j$ is absorbed into the $b b j$ component). Each sample is weighted by $x_{\text {sample }}$, the unknown fraction of multijet events contributed by that sample before b-tagging. The number of parameters was reduced by making the following assumptions: 


\begin{tabular}{|l|c|c|}
\hline & Number of events & $\begin{array}{c}\text { Fraction relative } \\
\text { to previous level }\end{array}$ \\
\hline Events with $>1$ MVA tight $b$-tag jet & $2,516,667$ & - \\
Fire $\phi b \bar{b}$ trigger & $1,506,807$ & 0.599 \\
$>2$ good, taggable jets & $1,206,319$ & 0.800 \\
$>2$ MVA tight $b$-tag jets & 40,044 & 0.033 \\
$\Delta R_{\text {(jet } 2, \text { jet 3) }>1.2}$ & 31,167 & 0.778 \\
with 3 jets (exclusive) & 18,164 & 0.583 \\
with 4 jets (exclusive) & 13,003 & 0.417 \\
\hline
\end{tabular}

Table 3.6: The number of events and relative fraction of events in data passing each cut in RunIIb. Note that events with five or more jets are not included in this table.

- $x_{b j j}=x_{c j j}, x_{b b j}=x_{c c j}$ and $x_{b b b}=x_{c c c}$. The difference in mass between b-jets and c-jets becomes negligible at high $H_{T}$, so $c j j, c c j$ and $c c c$ events can be grouped with the $b j j, b b j$ and $b b b$ events respectively.

- $x_{b b b}=x_{b b c}=x_{b c c}$. The production cross section of the $b b c c$ process was found to be twice that of $b b b b$ in ALPGEN. The third jet in $b b c c$ was as likely to be a b-jet as it was a c-jet, so bbcc was split equally between $b b c$ and $b c c$ events. For this reason, the three contributions were considered to be equal.

This reduced the number of free, independent parameters to three: $x_{b b b}, x_{b b j}$ and $x_{b j j}$. These, together with the $x_{j j j}$ contribution, sum to unity. These values were then multiplied by the b-tagging efficiency (measured by the D $\varnothing$ b-ID group [76]) for the relevant sample, denoted $\epsilon_{\text {sample }}$, to define a new set of parameters:

$$
X_{\text {sample }}=x_{\text {sample }} \times \epsilon_{\text {sample }}
$$

Taking the efficiency into account in this way, the final parameters were:

- $X_{b b b}=\frac{\epsilon_{b}}{\epsilon_{c}} X_{b b c}=\left(\frac{\epsilon_{b}}{\epsilon_{c}}\right)^{2} X_{b c c}=\left(\frac{\epsilon_{b}}{\epsilon_{c}}\right)^{3} X_{c c c}$

- $X_{b b j}=\left(\frac{\epsilon_{b}}{\epsilon_{c}}\right)^{2} X_{c c j}$

- $X_{b j j}=\frac{\epsilon_{b}}{\epsilon_{c}} X_{c j j}$

- $X_{j j j}$ 
where the relative b-tagging efficiencies of b- and c-jets $\left(\epsilon_{b} / \epsilon_{c}\right)$ were known. The b-tagging efficiency for b-jets was roughly five time that for c-jets, so the $X_{c c c}$ contribution was negligible. The remaining eight parameters were used to fit the MC samples to the data for 0-, 1-, 2- and 3-tagged jet cases. The sample contributions and b-tagging efficiencies varied with $H_{T}$ so the fit was carried out for individual bins in $H_{T}$ to allow for this. For each $H_{T}$ bin, the number of events in each sample were weighted by the relevant (unknown) $X_{\text {sample }}$ parameter, and the total was equated to the number of events for data, giving a set of linear equations for each bin [66]. The values of $X_{\text {sample }}$ that gave the best fit to the data were used to scale the MC samples: the MC-data agreement in the $H_{T}$ distribution is shown for the RunIIb three jet channel as an example in Figures 3.4 and 3.5, on linear and logarithmic scales respectively. The contributions of each MC sample as a fraction of the total MC background in the three jet channel are given in Tables 3.7 to 3.8: these tables give the integral of the $H_{T}$ distribution of each sample divided by the integral of the total $\mathrm{MC} H_{T}$ distribution.

In the four jet channel, the samples were scaled using the relevant value of $X_{\text {sample }}$ for the leading three jets and scaling this parameter by an extra factor to account for the extra jet (this factor was a free parameter in the fit). In the RunIla data, this factor was close to one, so the parameter values for the four jet channel were the same as those in Table 3.7. The contributions for the RunIIb four jet channel are given in Table 3.9.

In addition to the multijet background, $t \bar{t}$ events were simulated in MC. The $t \bar{t}$ contribution was almost negligible for the three-jet channel, and less than five percent for the four-jet channel. The contributions of processes with lower cross sections, such as $Z \rightarrow \tau^{+} \tau^{-}$, were considered to be negligible and were not included.

\begin{tabular}{|l|l|l|l|l|}
\hline \multirow{2}{*}{ Process } & \multicolumn{3}{|c|}{ \% Composition (Run IIa) } \\
\cline { 2 - 5 } & 0-tag & 1-tag & 2-tag & 3-tag \\
\hline$b b b$ & 0.1 & 0.3 & 2.7 & 51.2 \\
$b b c+b c c$ & 0.2 & 0.9 & 3.0 & 15.1 \\
$b b j$ & 3.7 & 19.0 & 82.3 & 30.7 \\
$c c j$ & 4.1 & 8.6 & 5.1 & 1.8 \\
$j j j+c j j+b j j$ & 92.0 & 71.2 & 6.9 & 1.2 \\
\hline
\end{tabular}

Table 3.7: Contributions of MC processes to the total MC multijet 3-jet background (RunIIa). The error on these values is of the order of $5 \%$. 

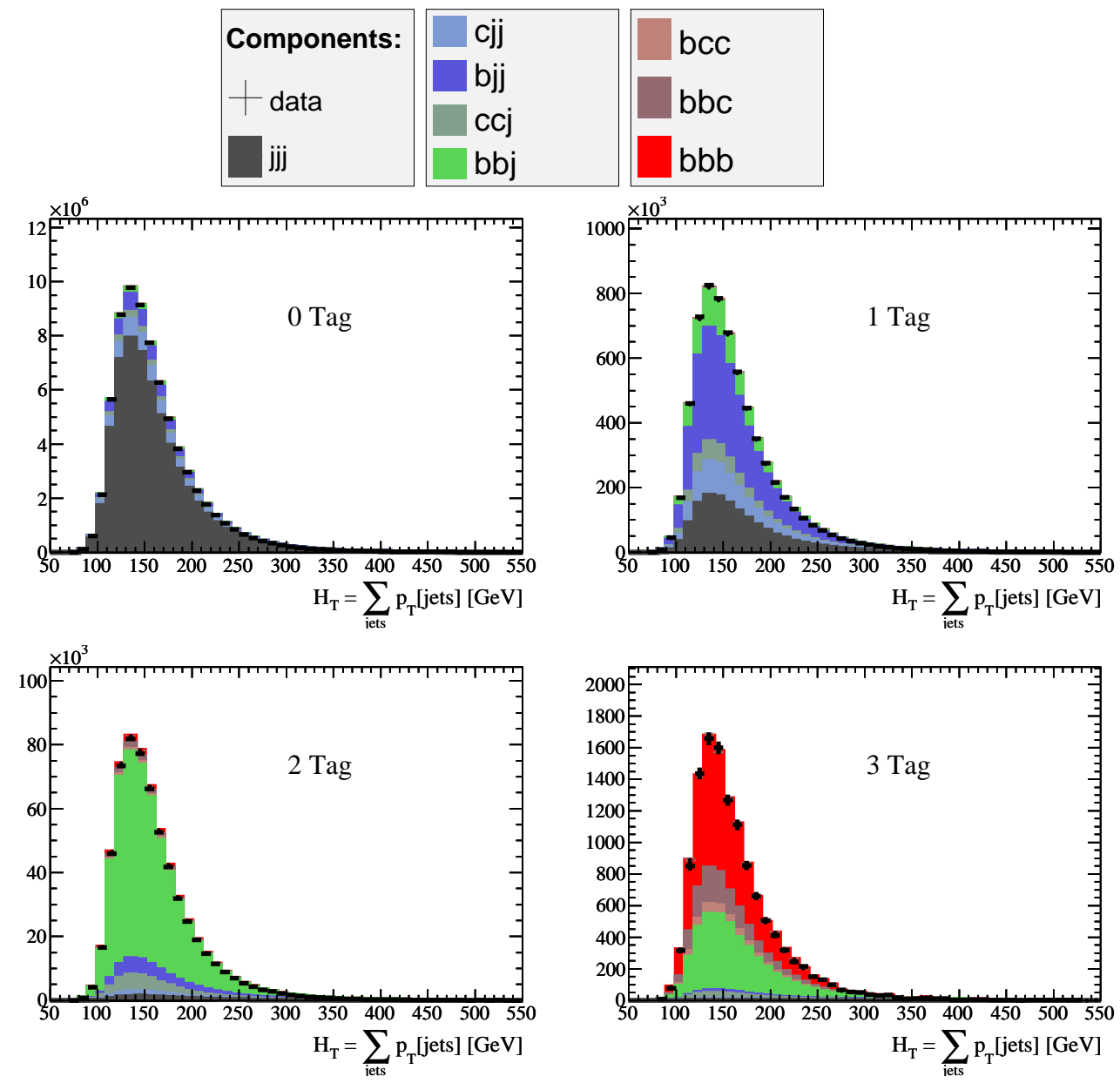

Figure 3.4: Composition of zero to three $b$-tagged three jet data samples for RunIIb. Numerical values for each of the $b$-tagged samples are shown in Table 3.8. This method was also applied to four-jet data and RunIIa data.

\begin{tabular}{|l|l|l|l|l|}
\hline \multirow{2}{*}{ Process } & \multicolumn{3}{|c|}{ \% Composition (Run IIb) } \\
\cline { 2 - 5 } & 0-tag & 1-tag & 2-tag & 3-tag \\
\hline$b b b$ & 0.1 & 0.2 & 2.5 & 47.1 \\
$b b c+b c c$ & 0.1 & 0.6 & 3.1 & 16.5 \\
$b b j$ & 2.3 & 13.0 & 76.6 & 31.8 \\
$c c j$ & 2.5 & 7.0 & 6.3 & 2.4 \\
$j j j+c j j+b j j$ & 95.0 & 79.1 & 11.5 & 2.2 \\
\hline
\end{tabular}

Table 3.8: Contributions of MC processes to the total MC multijet 3-jet background (RunIIb). The error on these values is of the order of $5 \%$. 

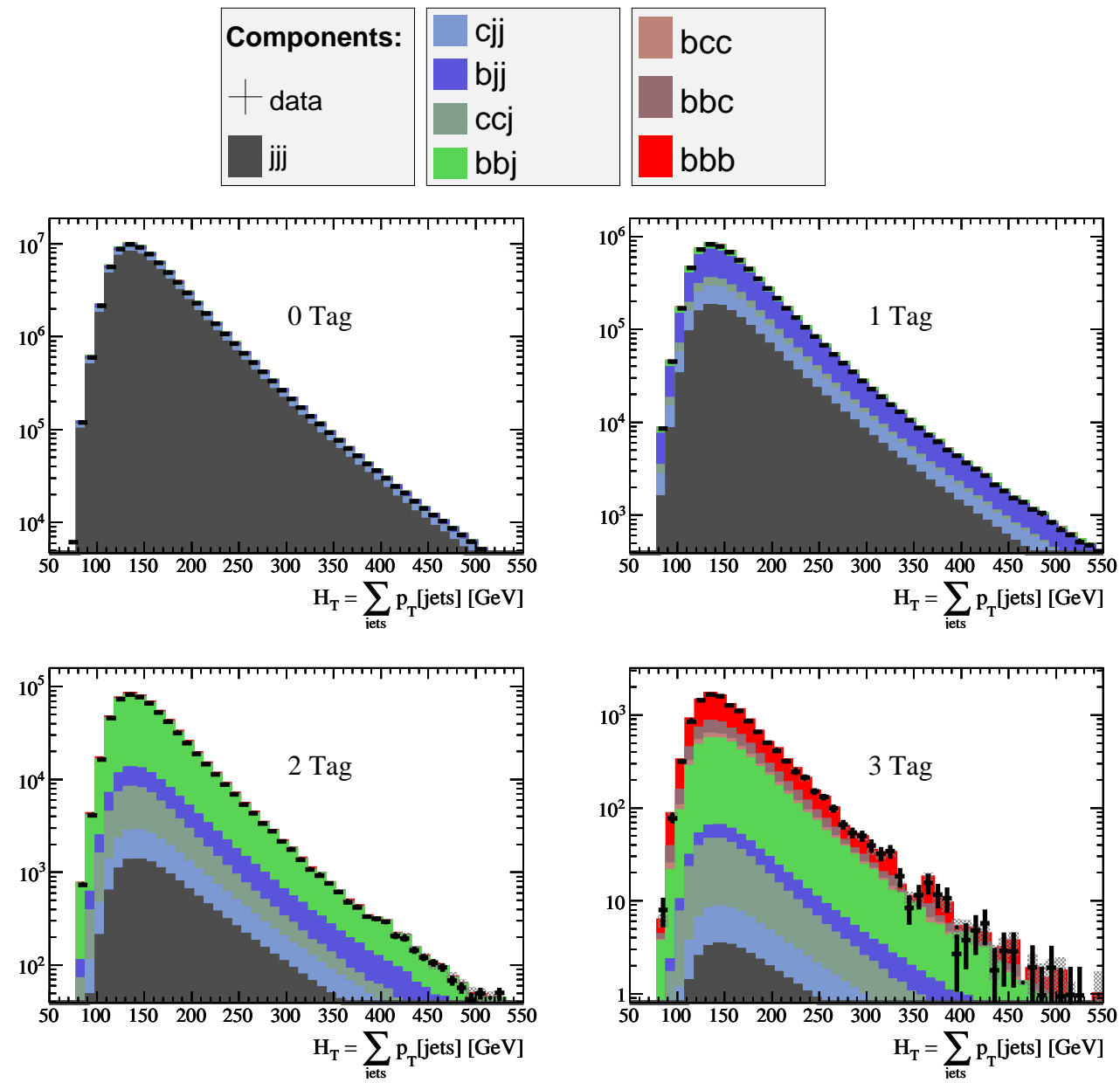

Figure 3.5: Composition of zero to three $b$-tagged three jet data samples for RunIIb plotted on a logarithmic scale. Numerical values for each of the $b$-tagged samples are shown in Table 3.8. This method was also applied to four-jet data and RunIIa data. The total MC background $H_{T}$ distribution agrees well with that for the data: this was achieved by fitting the MC to data for each individual bin in the $H_{T}$ distribution.

\begin{tabular}{|l|l|l|l|l|}
\hline \multirow{2}{*}{ Process } & \multicolumn{3}{|c|}{ \% Composition (Run IIb) } \\
\cline { 2 - 5 } & 0 -tag & 1-tag & 2-tag & 3-tag \\
\hline$b b b$ & 0.1 & 0.2 & 2.3 & 43.7 \\
$b b c+b c c$ & 0.2 & 0.7 & 3.2 & 20.1 \\
$b b j$ & 3.7 & 17.4 & 76.8 & 31.6 \\
$c c j$ & 4.1 & 9.4 & 6.8 & 2.5 \\
$j j j+c j j+b j j$ & 92.0 & 72.3 & 10.9 & 2.1 \\
\hline
\end{tabular}

Table 3.9: Contributions of MC processes to the total MC multijet 4-jet background (RunIIb). The error on these values is of the order of $5 \%$. 


\subsubsection{Background Prediction}

Although the MC samples can be fitted to data as described in Section 3.2.5, this method does not predict the shape or scale of the multijet background to the required degree of accuracy. For this reason, the background model was derived from data rather than using pure MC. Multijet events with 2 tight tagged jets are extremely common in data, and the signal contribution to the 2-tag data sample is negligible in comparison. The distribution in variable $x$ for 2-tag data can be deformed to that of a 3-tag sample using:

$$
\text { 3-Tag } \operatorname{Background}(x)=\frac{3-\operatorname{Tag} \operatorname{MC}(x)}{2-\operatorname{Tag} \operatorname{MC}(x)} \times 2-\operatorname{Tag} \operatorname{Data}(x)
$$

The background for each channel is calculated using the relevant (3-tag and 2tag) MC and data samples. All events contain at least three tight b-tagged jets, so distributions of all variables can be treated in this manner: however, the distributions must be calculated separately. This method relies on the fact that the main difference between the 3-tag and 2-tag MC samples is the b-tagging, so many unknown parameters cancel out in the ratio, allowing the shape of the background distribution to be determined.

The relative scales of 2-tag and 3-tag MC samples, however, were not necessarily correct, so this method could not accurately predict the background normalisation. For this reason, the normalisation was left as a free parameter when confidence limits were calculated.

The jet $\eta$ and $p_{T}$ distributions for the data, pure MC background and predicted background are shown in Figures 3.6 to 3.9 (the plots show RunIIb distributions as an illustration). The background has been normalised to data to aid comparison. The plots show that, in general, the predicted background is in reasonable agreement with the data, and is a better model than the 3-tag background simulated using pure MC. 

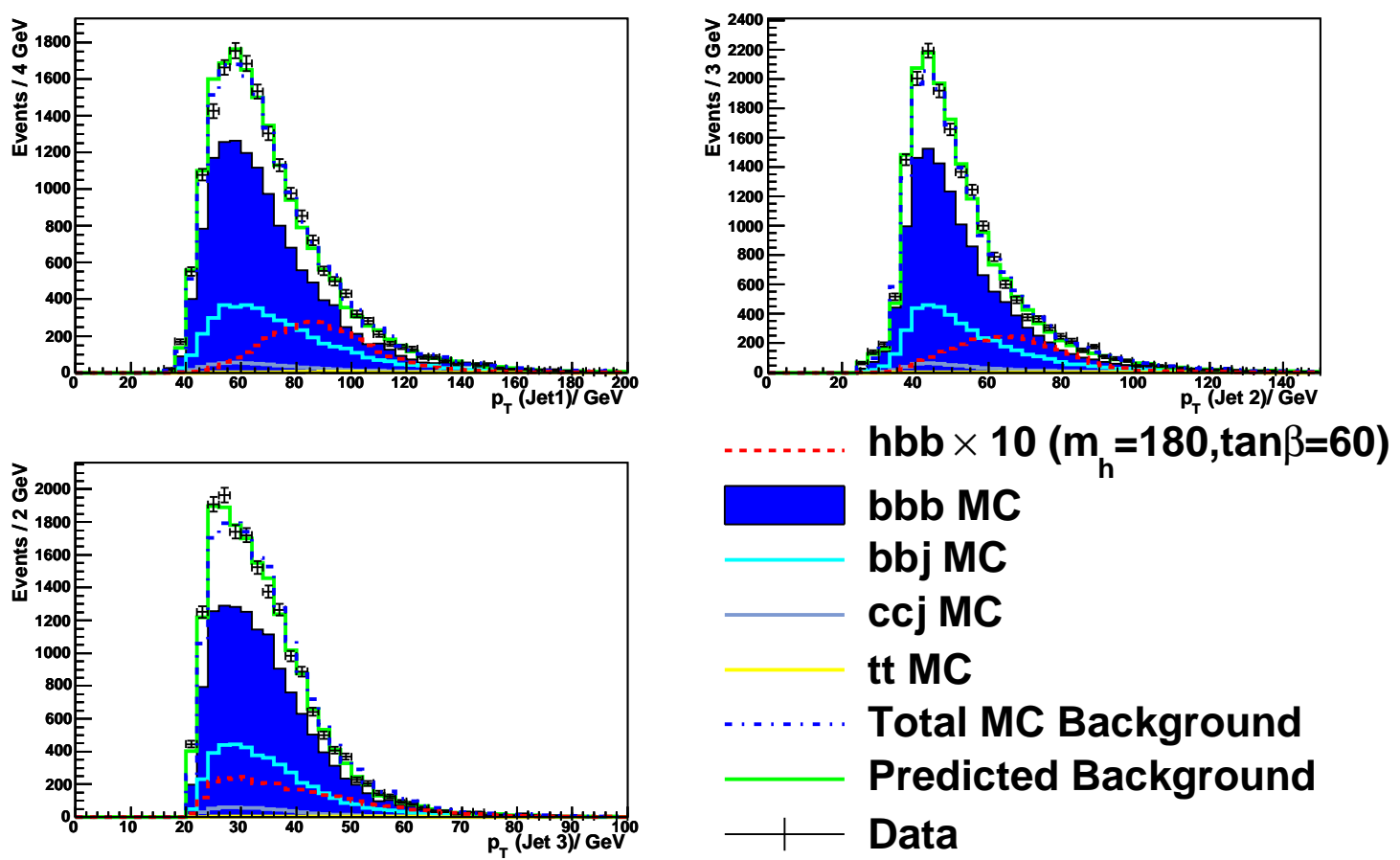

Figure 3.6: The transverse momentum $\left(p_{T}\right)$ distributions for the leading (top left), second (top right) and third (bottom left) jets in $p_{T}$ order for p20 data in the 3 -jet channel.
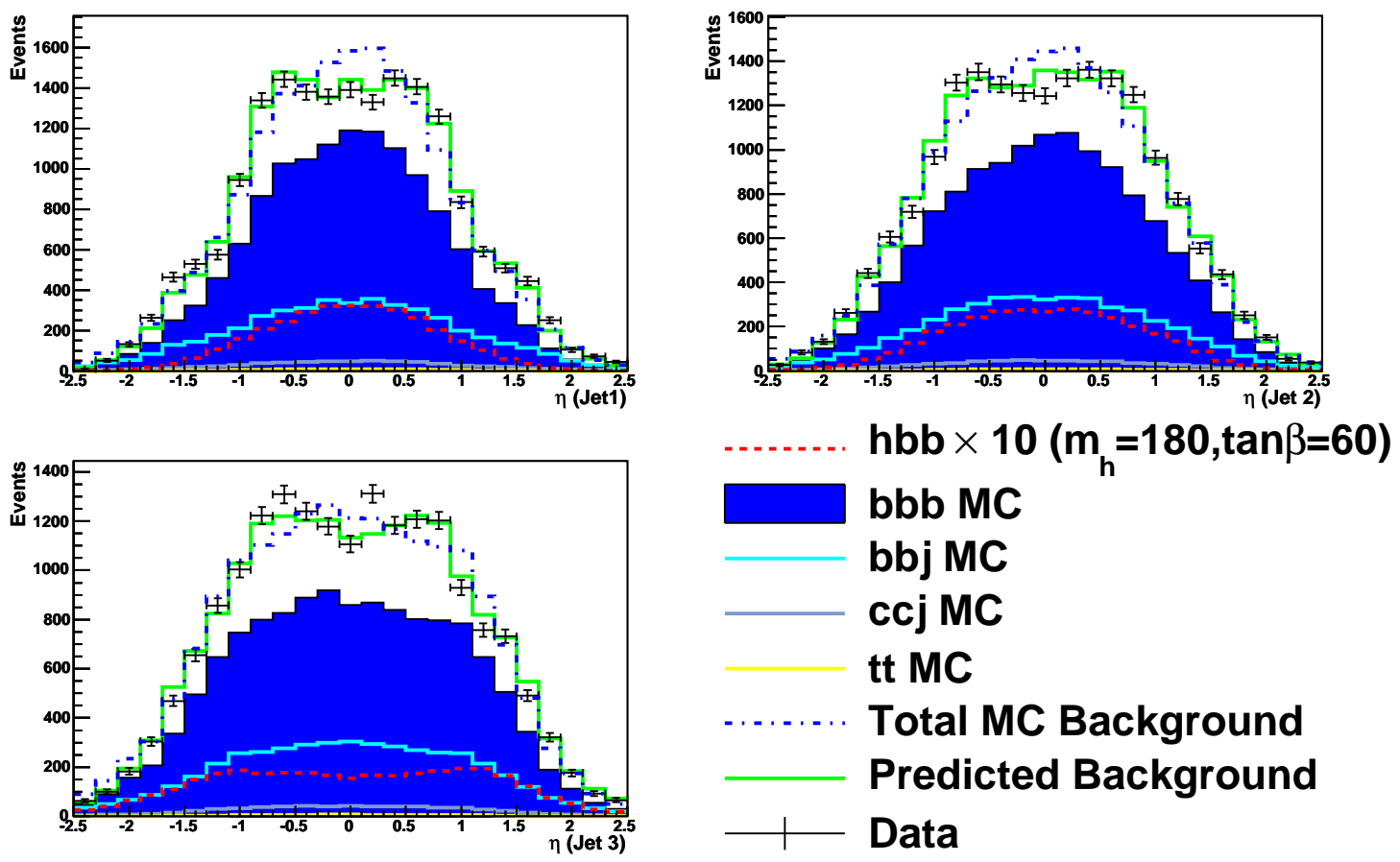

Figure 3.7: The pseudorapidity $(\eta)$ distributions for the leading (top left), second (top right) and third (bottom left) jets in $p_{T}$ order for p20 data in the 3 -jet channel. 

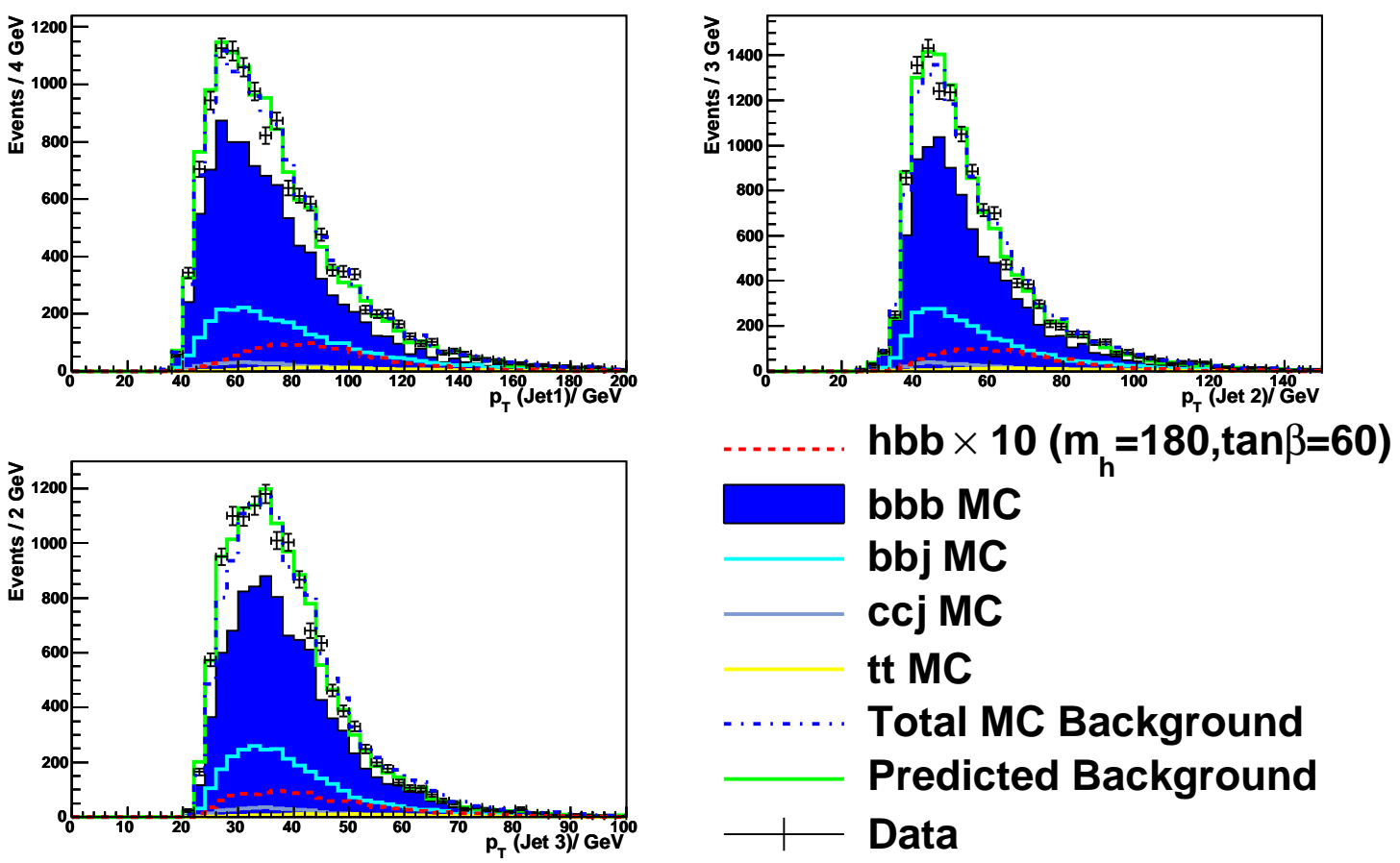

Figure 3.8: The transverse momentum $\left(p_{T}\right)$ distributions for the leading (top left), second (top right) and third (bottom left) jets in $p_{T}$ order for p20 data in the 4-jet channel.
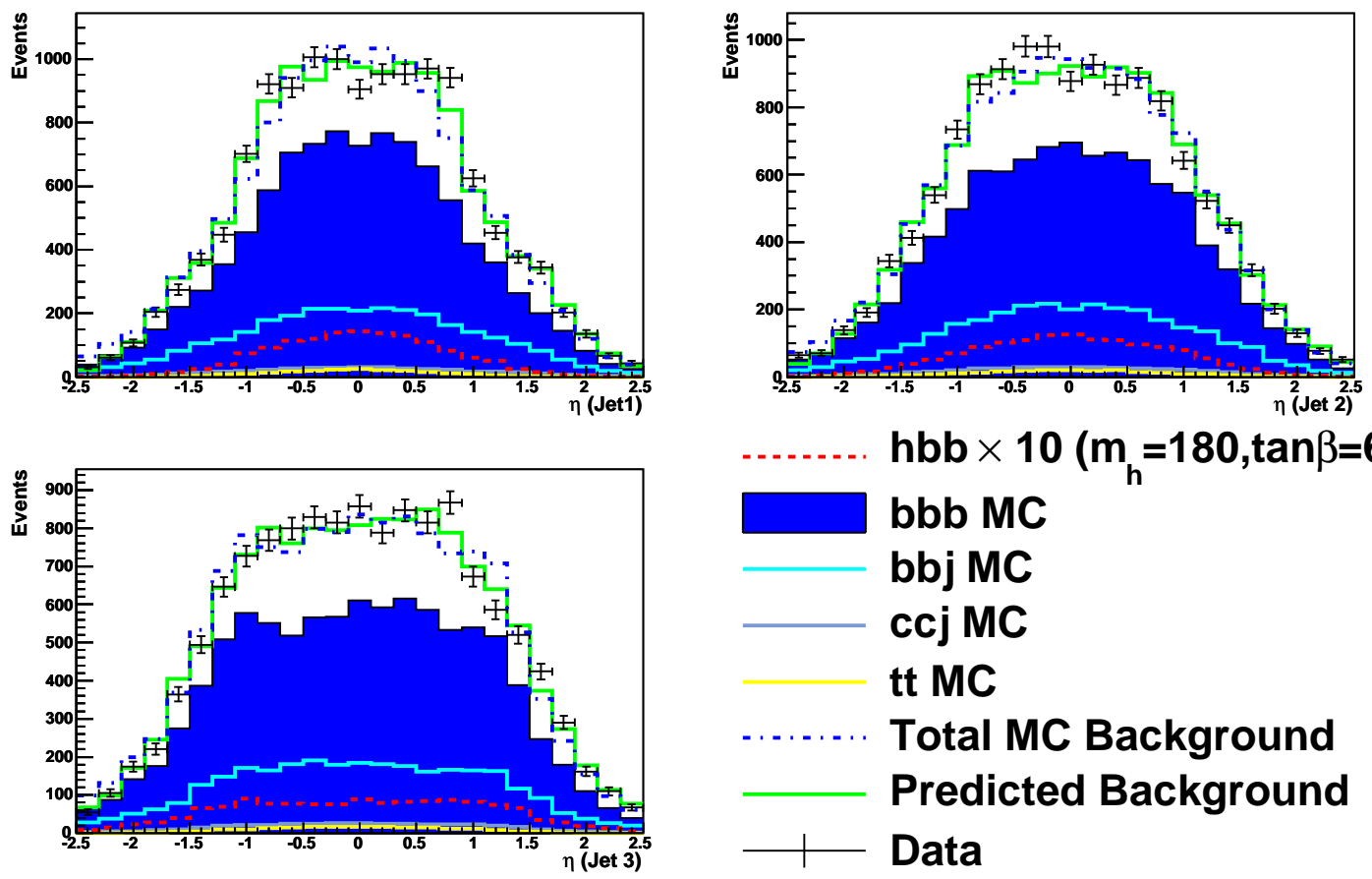

Figure 3.9: The pseudorapidity $(\eta)$ distributions for the leading (top left), second (top right) and third (bottom left) jets in $p_{T}$ order for p20 data in the 4-jet channel. 


\subsection{Conclusion}

The enhancement on the value of $\sigma \times B R$ for the process $\phi b \rightarrow b \bar{b} b$ makes searches for this signature a sensitive test of physics beyond the Standard Model. However, modelling the background processes for this channel also presents a significant challenge. The background was modelled in two steps: firstly the contribution of various processes to the total background were found by fitting them to data for different numbers of tight b-tags. Then the three tight b-tagged background distribution for variable $x$ was found by multiplying the two tight b-tagged data by a ratio derived from MC. This was found to give reasonable agreement with the data. 


\section{Chapter 4}

\section{Overview of Multivariate Methods for the Multijet Analysis}

\section{$4.1 \quad$ Introduction}

Having defined the background and signal samples and achieved acceptable agreement between the data and the background, variables that separated signal and background samples (discriminants) could be determined. The results of the search were determined using a hypothesis test: the distribution of the chosen discriminant variable was predicted for signal and background hypotheses and compared to the distribution of the discriminant in data. In general, the analysis is more sensitive if the discriminant used for setting limits (the final discriminant) is some combination of several discriminant variables: processes that perform this combination are called multivariate analysis (MVA) techniques. The strategy employed in this analysis was to use kinematic variables from three Higgs boson candidates (b-jet pairings) per event as inputs to the MVA. In the three jet channel, this simply meant all possible pairings of b-jets, whereas in the four jet channel only pairings of the leading three jets were considered (the Higgs boson is likely to decay to high energy jets, so it is assumed to be unlikely for the fourth jet in $p_{T}$ order to be derived from a Higgs boson decay). The input variables considered were:

- di-jet invariant mass

- $\Delta \eta\left(\left|\eta_{i}-\eta_{j}\right|\right)$

- $\Delta \phi\left(\left|\phi_{i}-\phi_{j}\right|\right)$

- momentum Balance $\left(\left|\frac{p_{i}-p_{j}}{p_{i}+p_{j}}\right|\right)$ 
- rapidity

- opening angle between Higgs boson candidate and jet $i$

for each of the three pairings, and the event sphericity ( $i$ and $j$ denote the leading and second leading jet in the pair respectively). This choice of variables was based on a study from the $1 \mathrm{fb}^{-1}$ analysis [77], and their effectiveness was verified by the author.

A number of MVA methods were tested for this analysis; a comparison of these methods is given in this Section. Each MVA was trained using MC events. One quarter of the events were used for training, and a further quarter were used for testing the technique (the remaining half were used for the analysis). Each method was trained on the same sample. For all methods, the events were weighted according to their cross sections (and in the case of the background $\mathrm{MC}$, the composition values obtained in Section 3.2.5), and then scaled such that the weighted total number of background events was 1, and the weighted total number of signal events was 1. For simplicity, the event weightings will not be included in the following discussion.

As well as providing a good discriminant, the output of the MVA was required to show consistency (that is, if the MVA were trained twice with the same input variables and training sample, it would give similar results each time).

\subsection{Neural Networks}

A neural network $(\mathrm{NN})$ is a pattern recognition algorithm which may be visualised as a network of nodes, arranged in layers, connected by edges (see Figure 4.1). In addition to the input and output layers, there may be a number of hidden layers between the two. An input node is associated with each input variable (denoted $x_{1}$ to $x_{n}$ for $n$ variables). The edges connecting these nodes to those in the next layer of the network (denoted $y_{1}$ to $y_{m}$ ) are associated with a weight, $w_{i j}$. The values of the nodes $y_{j}$ are given by a function of those in the previous layer and the weight as follows:

$$
y_{i}=F\left(w_{i j}, x_{j}\right)
$$

where

$$
F\left(w_{i j}, x_{j}\right)=w_{i j} x_{j}
$$

for nodes between input (or output) nodes and hidden nodes, and 


$$
F\left(w_{i j}, x_{j}\right)=\left(1+e^{w_{i j} x_{j}}\right)^{-1}
$$

for edges connecting hidden nodes.

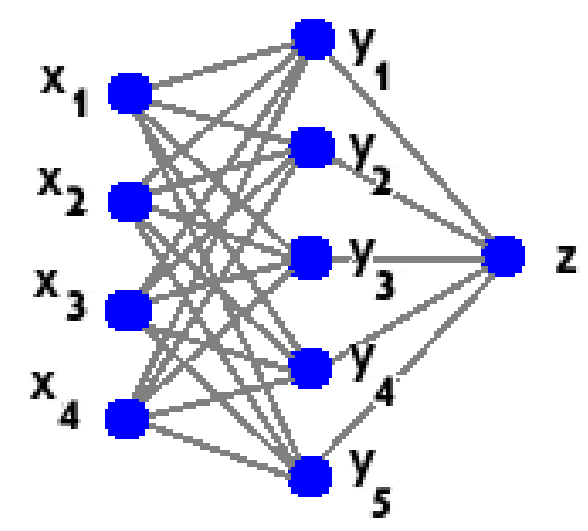

Figure 4.1: Diagram illustrating the structure of a simple neural network. The illustrated NN consists of three layers of nodes (illustrated as blue circles): an input layer (left), a hidden layer (centre) and an output layer (right). The edges which link the nodes are also shown.

The NN is trained to recognise signal and background events by a training algorithm. Initially the weights, $w_{i j}$, are randomised, and input variables for the data are fed into the network. The training data includes an indicator value, $I$; this is set to, for example, 1 in signal events and 0 in background events. The value of the output node $\left(I^{\prime}\right)$ is calculated for each event, and compared with the indicator value to give the error for each event, and the overall error is the sum of $\left(I-I^{\prime}\right)^{2}$ over all events. This error is a function of the weights $w_{i j}$, and is minimised using an iterative method. These iterations are known as epochs. There are several iterative methods: the method used in this study was the Broyden, Fletcher, Goldfarb, Shanno (BFGS [78]) algorithm, which uses Newton minimisation.

If the network is trained for too many epochs, the network may become too specific to the training sample. This is known as over-training, and is prevented by calculating the error $\left(I-I^{\prime}\right)^{2}$ for an independent sample of events (known as a testing sample) and comparing it with that of the training sample. If the errors diverge, the network is over-trained.

The NN algorithm contained in the class TMultiLayerPerceptron [79] was used in this analysis. When applied to the $\phi b \rightarrow b \bar{b} b$ analysis, the neural network method was found to be very unstable. There were large variations from one mass hypothesis to the next; in fact training several networks for a single mass hypothesis led to 
several different outcomes. This may have been due to the random starting point of the process, or the presence of false minima in the error function. The expected limits on $\tan \beta$ (used here to compare sensitivity) calculated using the NN output as the final discriminant are shown in Figure 4.5, and illustrate the inconsistency from one mass hypothesis to the next.

\subsection{Forest of Decision Trees with Boosting (BDT) Method}

A decision tree (DT) is a multivariate technique which uses a series of simple cuts to discriminate signal events from background. Starting with a mixed signalbackground sample, the DT evaluates a number of cuts for each variable to find the best cut for distinguishing signal from background. This cut is used to divide (split) the sample into two subsets. The next step is determined by the purity of the resulting subsets. The purity is defined as

$$
p=\frac{s}{s+b}=1-\frac{b}{s+b}
$$

Where $s$ and $b$ are the number of signal and background events, scaled such that $s+b=1$. This is used to calculate a separation criterion: in this case, the Gini index $(p(1-p))$ was used. A Gini index of 0.5 corresponds to a subset where $s$ and $b$ are equal. The Gini index decreases if $s$ is greater than $b$, or vice versa. If the separation criterion of a subset is below a pre-set threshold, no further splitting occurs for that subset. If the threshold is not passed, an optimised cut is then found for that subset, and it is split into two smaller subsets. This process continues until all subsets have reached the threshold purity or the maximum number of split levels have occurred (this maximum is a parameter of the algorithm). An output value is then assigned to each event: - 1 if the event is placed in a background-dominated subset, and +1 if it is placed in a signal-dominated subset. Figure 4.2 shows the structure of a simple decision tree.

This method will lead to several events being wrongly classified, leading to a loss in sensitivity. This can be mitigated by use of a boosting algorithm [80]. Boosting is carried out by training an ensemble of trees (known as a forest) on the same data sample and altering the weight of each event after each tree is trained. The events are re-weighted according to their classification: those that were classified correctly have their weight reduced, while those that were classified incorrectly are given an increased weight. When the splitting occurs in the next tree, the previously 


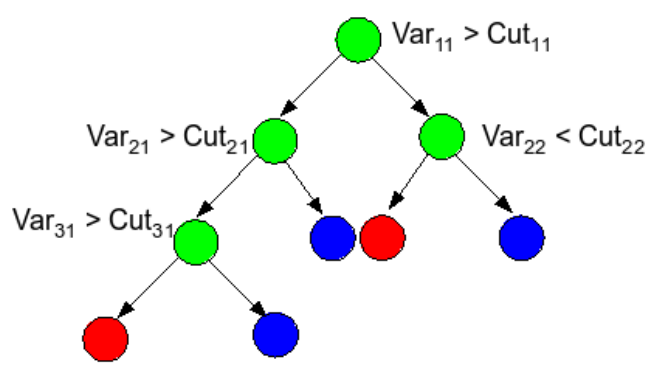

Figure 4.2: Diagram illustrating the structure of a simple decision tree (DT). The green circles represent samples that do not meet the purity criterion, while the red and blue circles are samples below the purity threshold, which are dominated by signal and background events respectively. At the first node (at the top of the diagram), the DT tests a number of possible cuts on each variable, and uses the cut which provides the best signal-background separation. This process is repeated until all samples reach the purity criterion, or the maximum depth of the tree is reached.

mis-identified events have a greater influence on the cut optimisation, and are more likely to be correctly identified. This produces a Forest of Boosted Decision Trees, abbreviated as BDT. The output of the BDT is a weighted average of the outputs of all DTs in the forest (the output of DTs trained later, which received more boosting, are weighted more strongly than those which were trained earlier). The ROOT TMultiVariateAnalysis (TMVA [81]) toolkit was used. Forests of 100 trees were trained to test the method.

This method was found to be unsuitable for the strategy described in Section 4.1. The signal sample may be divided into two main subsets: events where the Higgs boson daughter particles are the two leading jets, and those where they are the leading and third-leading jets. Signal events in the first case have similar properties to background events in the second, and vice versa. The boosting algorithm caused these two subsets to be weighted equally, reducing the discriminating power of the forest as the number of trees increased, as illustrated in Figure 4.3. The expected limits on $\tan \beta$ using the BDT output as the final variable are given in Figure 4.5; again, these showed large fluctuations from one mass hypothesis to the next. It would be possible to train two or three BDTs, with each BDT focussing on one pairing. A simpler method was chosen, however, as discussed in the next Section.

\subsection{Forest of Randomised Decision Trees (RF) Method}

An alternative method using decision trees is the Forest of Randomised Decision Trees, or Random Forest (RF) [82]. In a similar manner to the BDT method, a RF 


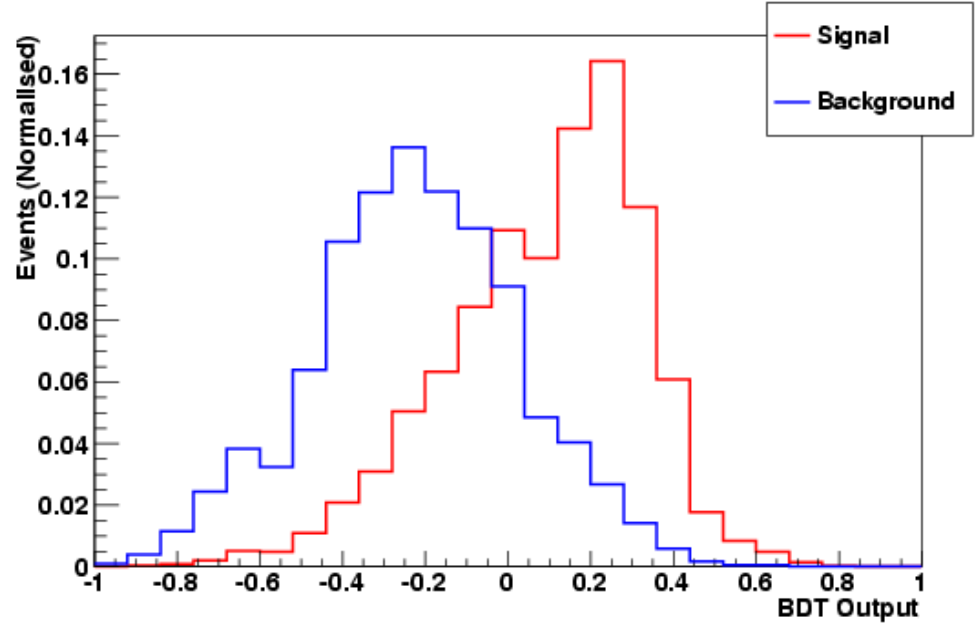

(a)

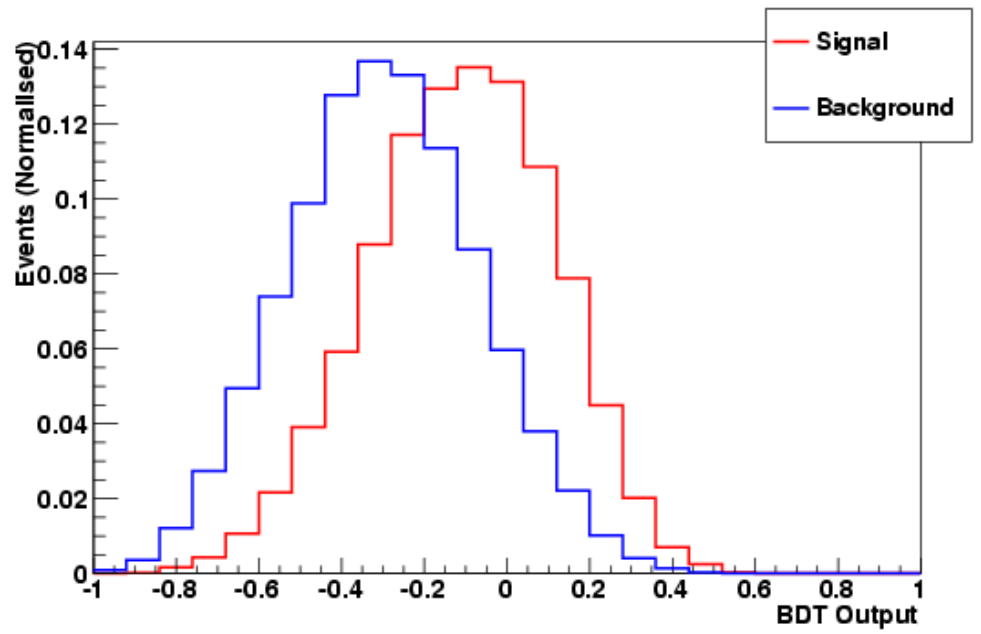

(b)

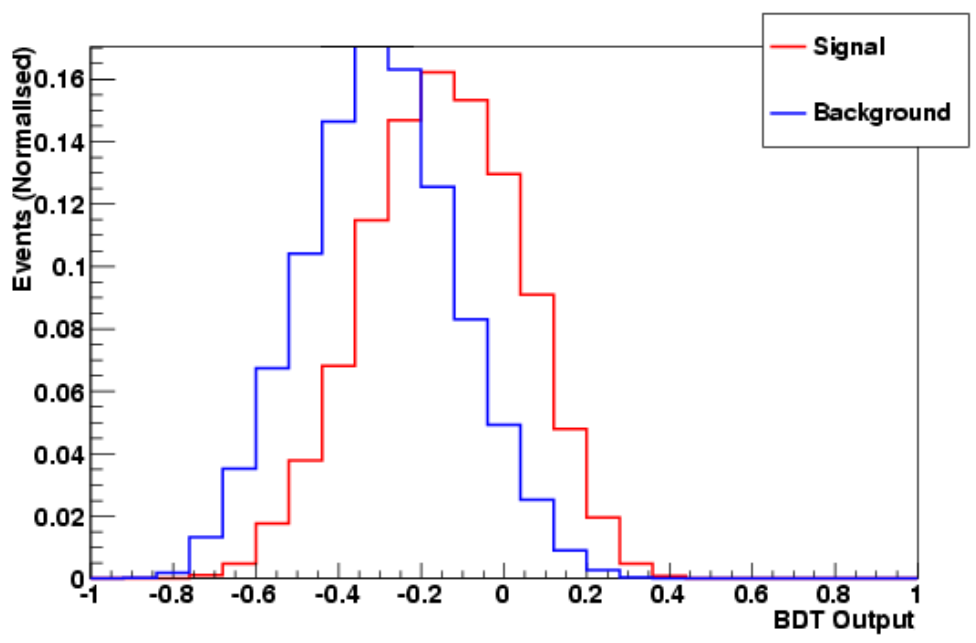

(c)

Figure 4.3: Output values of signal (red) and background (blue) MC samples for BDT method after (a) 20, (b) 50 and (c) 100 trees. The signal and background distributions converge as the number of trees increases. 
uses an ensemble of trees. The boosting method is exchanged for a process known as 'bagging': before each tree is trained, every event is randomly assigned a weight of one or zero. This means that each tree is trained over a random subset of the data. Additionally, a randomly chosen subset of input variables is used in each tree. This method allows good signal-background separation for different subsets of data, without using the boosting algorithm. The output of the RF is the average of the outputs of all DTs in the RF. The output becomes more consistent if larger numbers of trees are trained. To test the method, forests of 100 trees were trained.

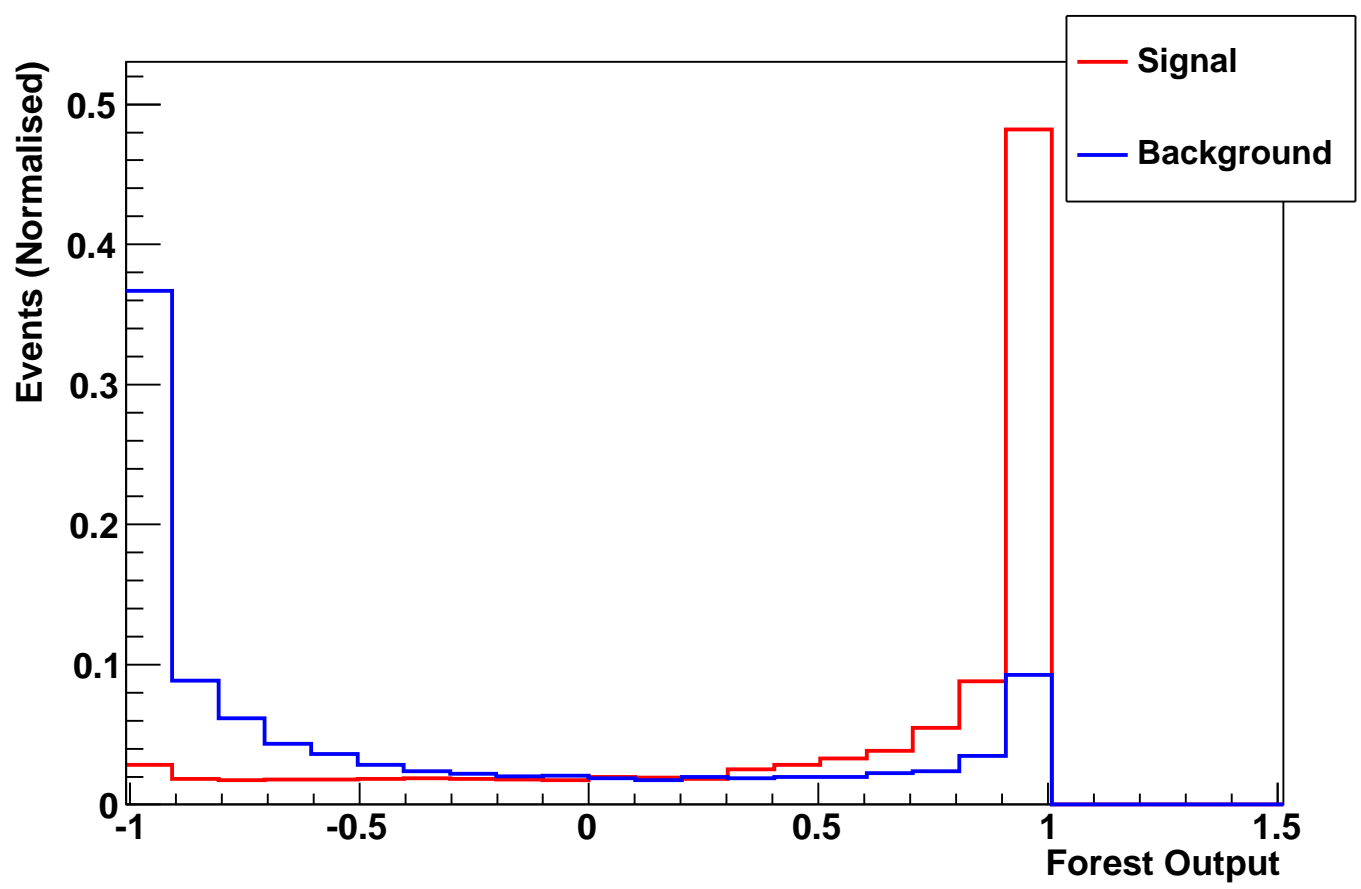

Figure 4.4: Random Forest output distributions for the signal (red) and background (blue) MC training samples. The signal and background RF output distributions peak at \pm 1 , indicating good discrimination between signal and background separation.

Figure 4.4 shows an example random forest output for MC signal and background. The output distributions demonstrate good signal-background separation. In addition, the RF was more stable than the other methods tested, as can be seen in the expected sensitivity illustrated in Figure 4.5. For this reason, the RF method was used in the analysis.

\subsection{Random Forest Input Variables and Outputs}

The performance of an MVA can be improved by careful choice of input variables. Variables which do not distinguish signal and background can detract from the 


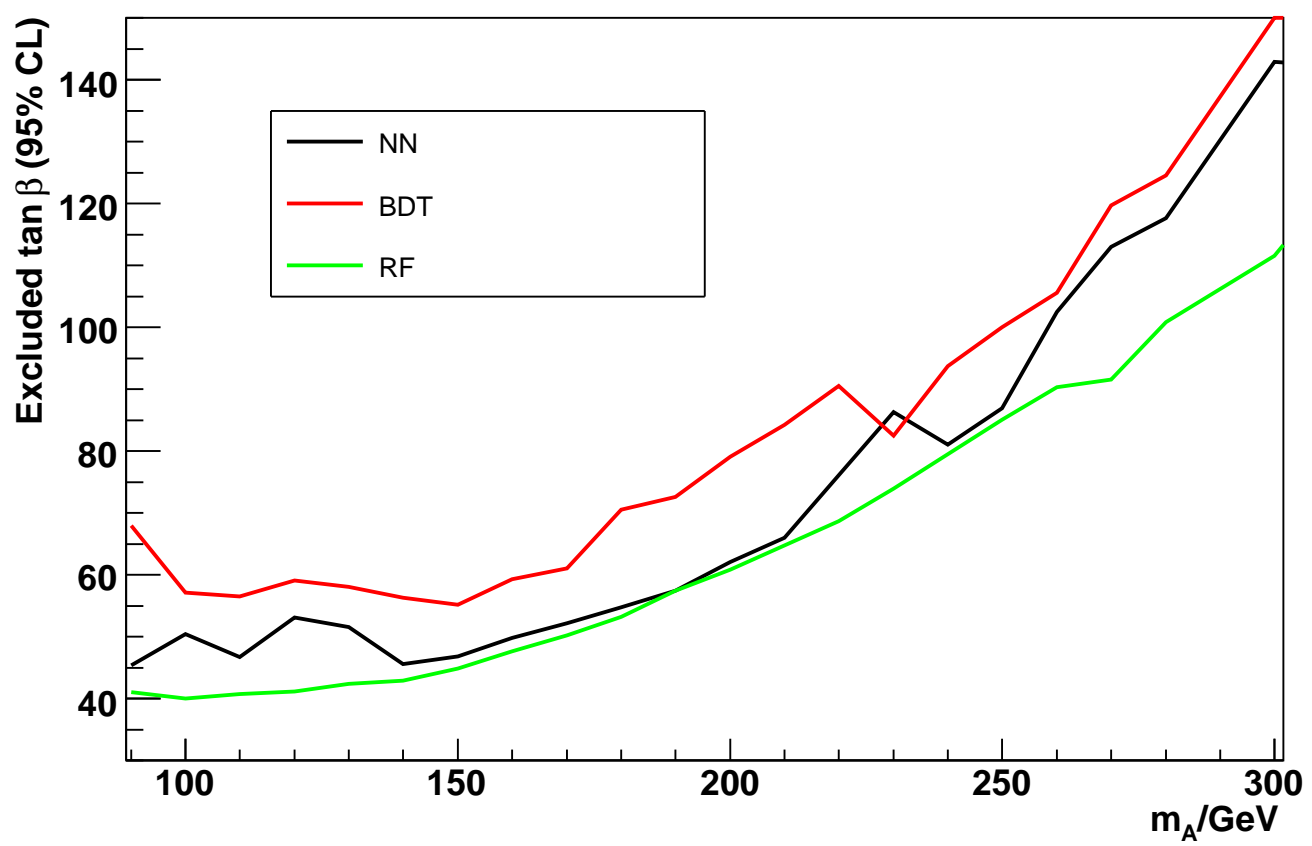

Figure 4.5: A comparison of expected limits on $\tan \beta$ using different MVA methods. The expected limits for the NN, BDT and RF methods are represented by the black, red and green lines respectively. For this comparison,all limits were set without systematic errors. The RF method was chosen because of its stability. The limit setting process is described in Chapter 5. 
overall performance of the MVA. The TMVA RF algorithm produces a list of the variables used at each splitting in each DT. Variables that appeared infrequently on the list were removed from the RF. An extra variable (the mass deviation angle) was added at this stage: this variable is defined and discussed below.

The following variables were found for each Higgs candidate (jets $i$ and $j$ ), and used as inputs for the RFs:

- di-jet invariant mass

- $\Delta \eta\left(\left|\eta_{i}-\eta_{j}\right|\right)$

- opening angle between Higgs boson candidate and the leading jet in the pair.

In addition to the pair-based variables, two event-based variables were included:

- event sphericity (three jet channel only)

- invariant mass deviation angle $\left(\arccos \left(\frac{\text { Mean Mass }}{\text { RMS Mass }}\right)\right)$ where the mean and RMS masses are the mean and RMS of the masses of the three Higgs boson candidates.

Therefore a total of 11 input variables were used in the three jet channel. The event sphericity was not modelled well in the four jet channel, so it was not used as an input for the four jet random forests.

The invariant mass deviation angle is a measure of how similar the masses of the three Higgs candidates are: the closer these masses are, the smaller the angle. The deviation angle for a given event is derived by plotting the event as a vector, $\vec{M}$, in (M12, M23, M13) space, where Mij is the invariant mass of jets $i$ and $j$. The deviation angle is the angle between this vector and the vector $\vec{a}=(1,1,1)$. This is illustrated in two dimensions in Figure 4.6. The scalar product rule gives the deviation angle as

$$
\begin{gathered}
\text { Deviation Angle }=\arccos \left(\frac{\vec{M} \cdot \vec{a}}{|\vec{M}||\vec{a}|}\right) \\
\text { Deviation Angle }=\arccos \left(\frac{M 12+M 23+M 13}{3} \times \sqrt{\frac{3}{M 12^{2}+M 23^{2}+M 13^{2}}}\right)
\end{gathered}
$$

This is identical to the expression given in the list of variables above. This value was found to be relatively low for low mass signal events, but high for background 


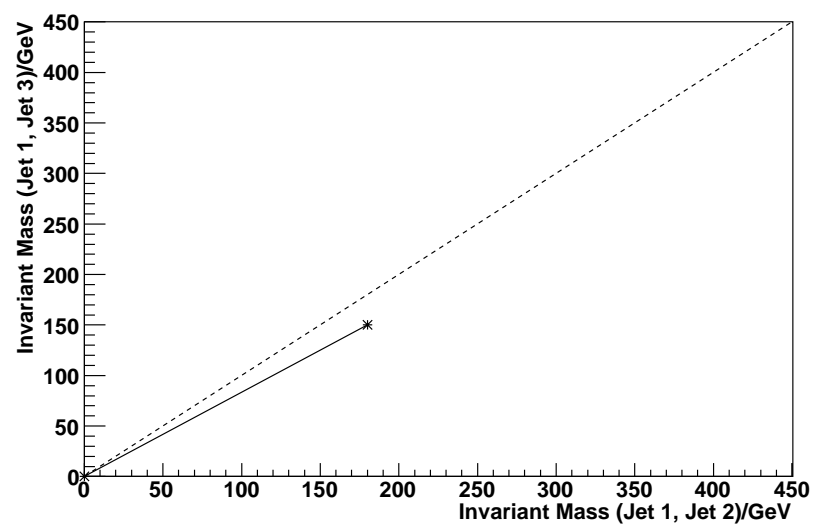

Figure 4.6: The deviation angle illustrated for two Higgs candidates: the leading and secondleading jet, and the leading and third-leading jet. The candidates have masses $180 \mathrm{GeV}$ and 150 $\mathrm{GeV}$ and are plotted on the $\mathrm{x}-$ and $\mathrm{y}$-axis respectively. Events where the two quantities are equal would lie on the dotted line. The angle between the dotted and solid black lines is a measure of deviation of both masses from the mean. A three-dimensional version of this angle was used in this analysis.

events: this was chosen in preference to the standard deviation because the possible range of values $(0-\pi / 4)$ was the same for all mass hypotheses.

The distributions of the input variables for the MC signal and background in the three jet channel are shown for low, medium and high Higgs boson mass hypotheses in Figures 4.7 to 4.11. The data-predicted background comparisons are shown in Figures 4.12 to 4.16 : these plots show that the predicted background matches the shape of the data distribution well (the predicted background has been normalised to equal area with data). The signal distribution in these histograms is for a Higgs boson of mass $180 \mathrm{GeV}$, unless stated otherwise. 

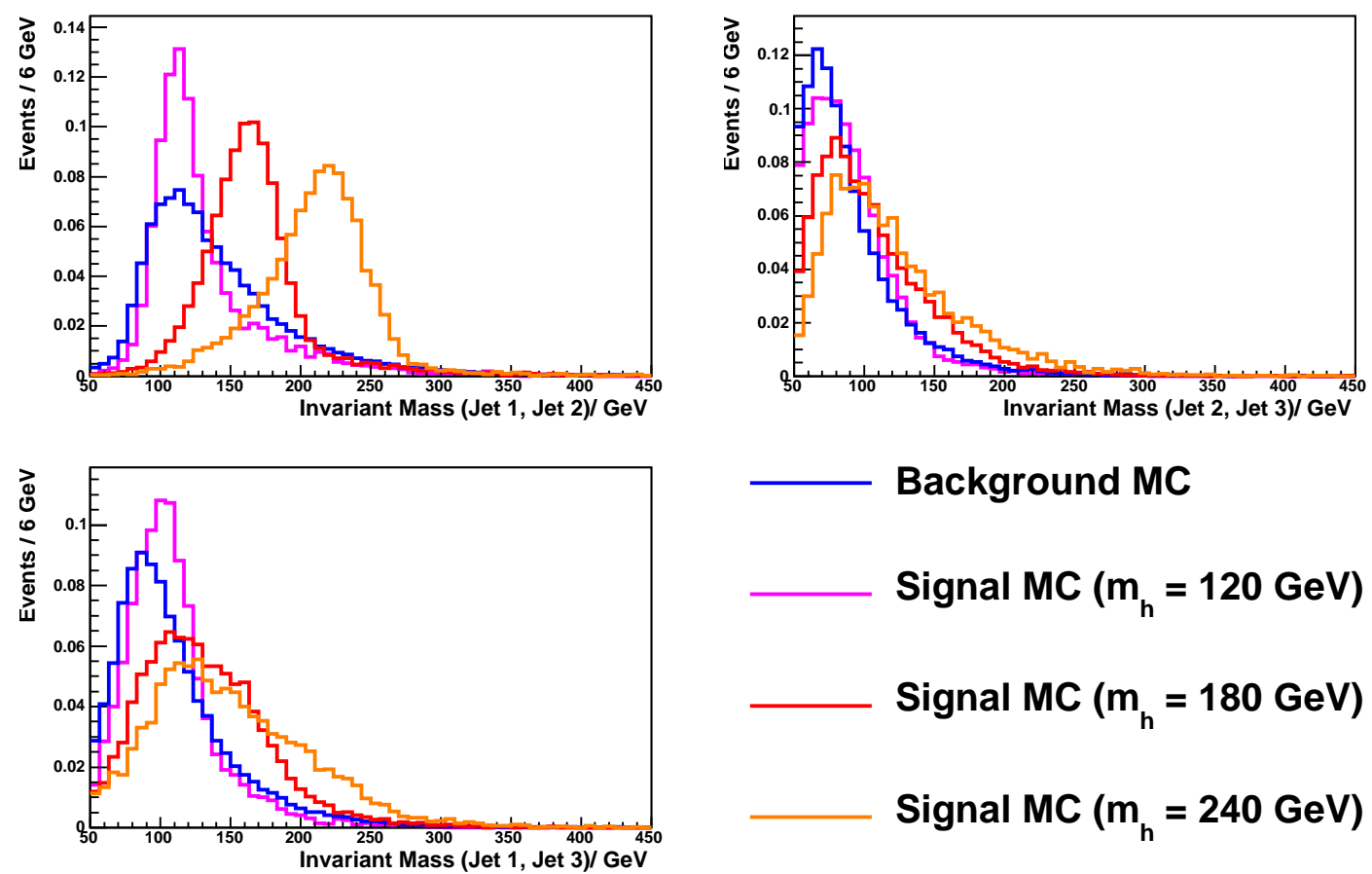

Background MC

Signal MC $\left(m_{h}=120 \mathrm{GeV}\right)$

Signal MC $\left(m_{h}=180 \mathrm{GeV}\right)$

Signal MC $\left(m_{h}=240 \mathrm{GeV}\right)$

Figure 4.7: The di-jet invariant mass distributions for jet pairs 12 (top left), 23 (top right) and 13 (bottom left) for p20 MC in the 3-jet channel. The blue histogram represents MC background, while the purple, red and orange histograms are signal distributions for $m_{h}$ of 120,180 , and 240 $\mathrm{GeV}$ respectively. 

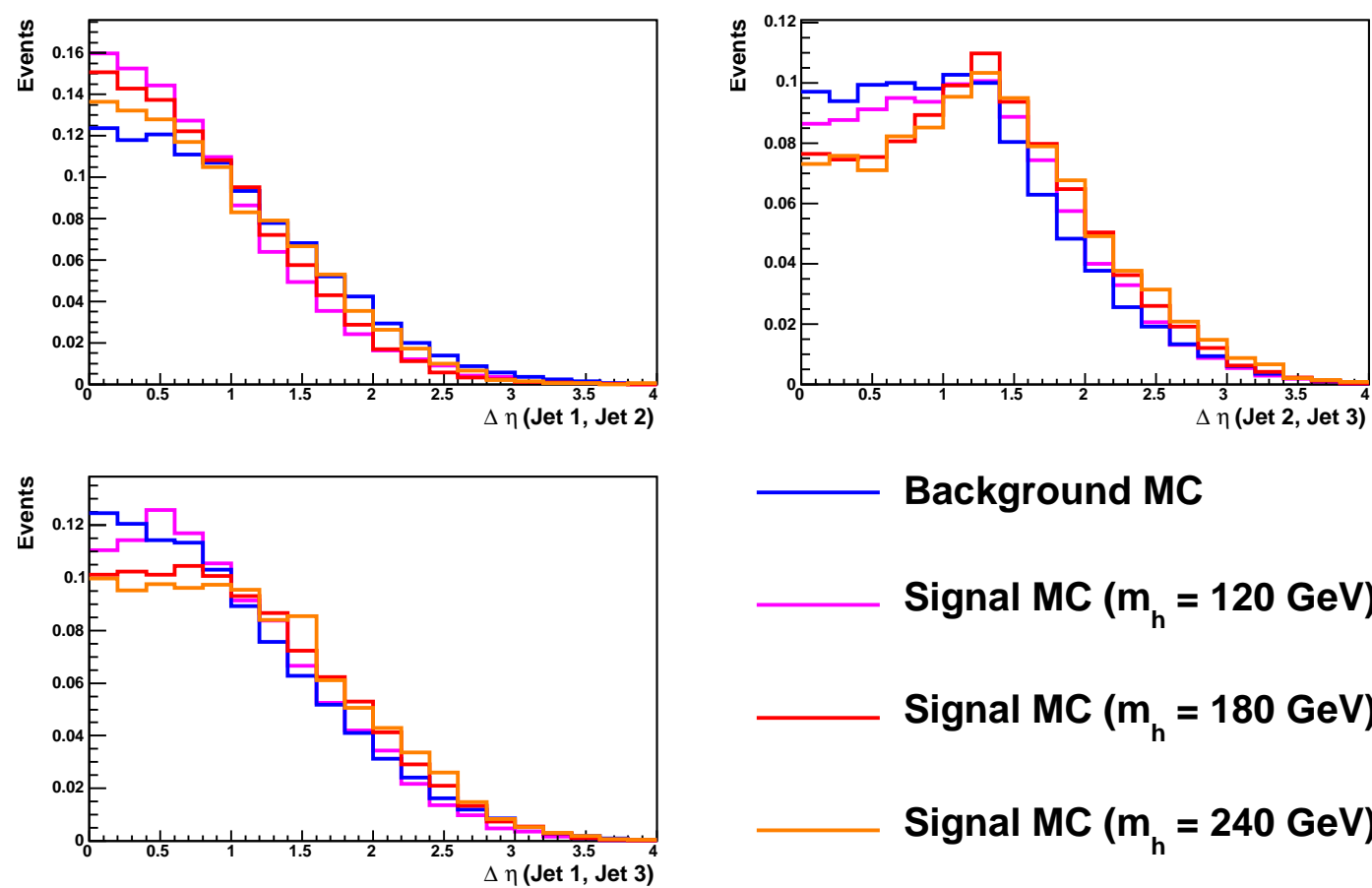

Background MC

Signal MC $\left(m_{h}=120 \mathrm{GeV}\right)$

Signal MC $\left(m_{h}=180 \mathrm{GeV}\right)$

Signal MC $\left(m_{h}=240 \mathrm{GeV}\right)$

Figure 4.8: The $\Delta \eta$ distributions for jet pairs 12 (top left), 23 (top right) and 13 (bottom left) for p20 MC in the 3-jet channel. The blue histogram represents MC background, while the purple, red and orange histograms are signal distributions for $m_{h}$ of 120,180 , and $240 \mathrm{GeV}$ respectively.
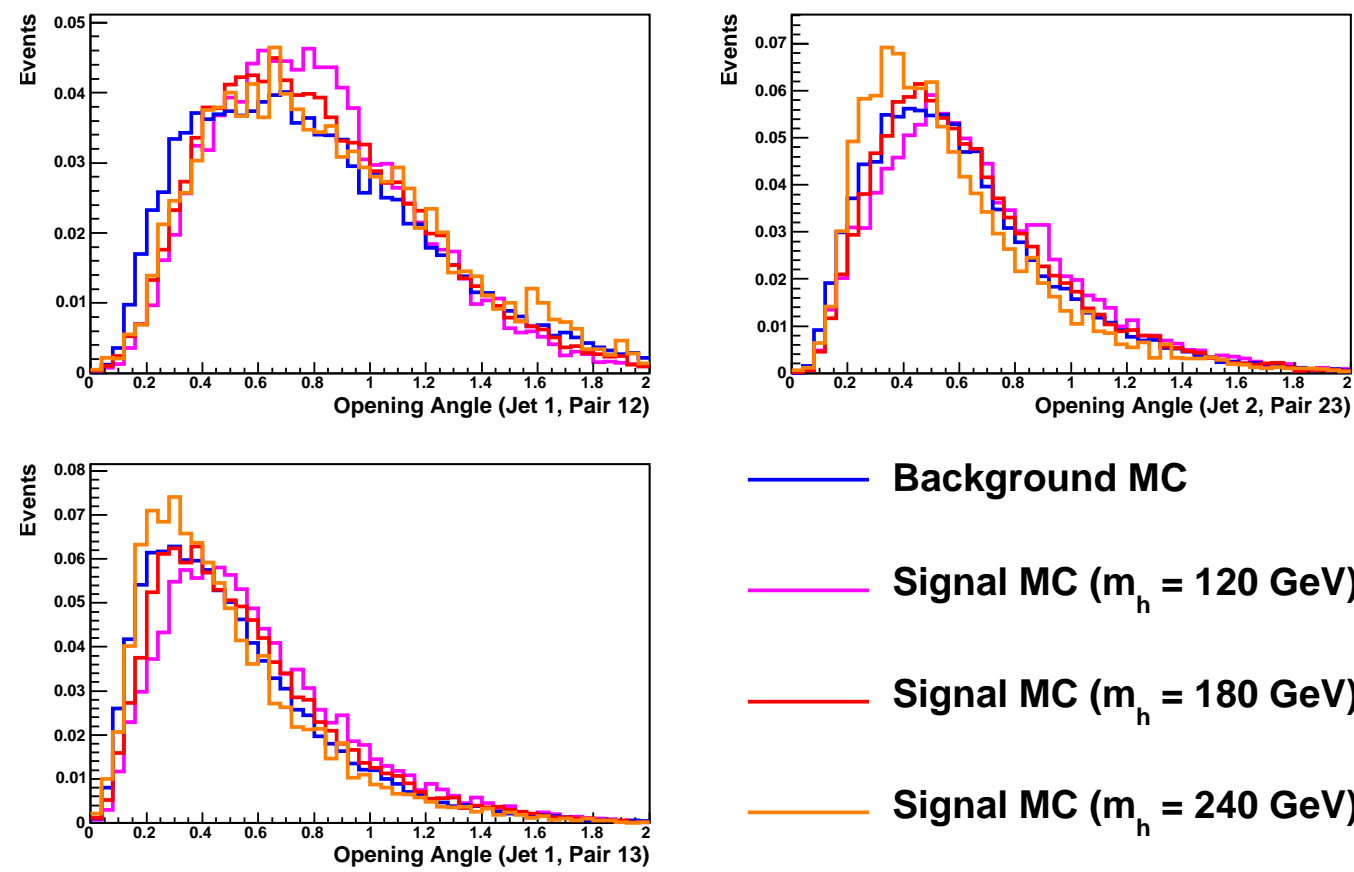

Background MC

Signal MC $\left(m_{h}=120 \mathrm{GeV}\right)$

Signal MC $\left(m_{h}=180 \mathrm{GeV}\right)$

Signal MC $\left(m_{h}=240 \mathrm{GeV}\right)$

Figure 4.9: The opening angle distributions for jet pairs 12 (top left), 23 (top right) and 13 (bottom left) for p20 MC in the 3-jet channel. This angle is described in the list of RF input variables. The blue histogram represents MC background, while the purple, red and orange histograms are signal distributions for $m_{h}$ of 120,180 , and $240 \mathrm{GeV}$ respectively. 


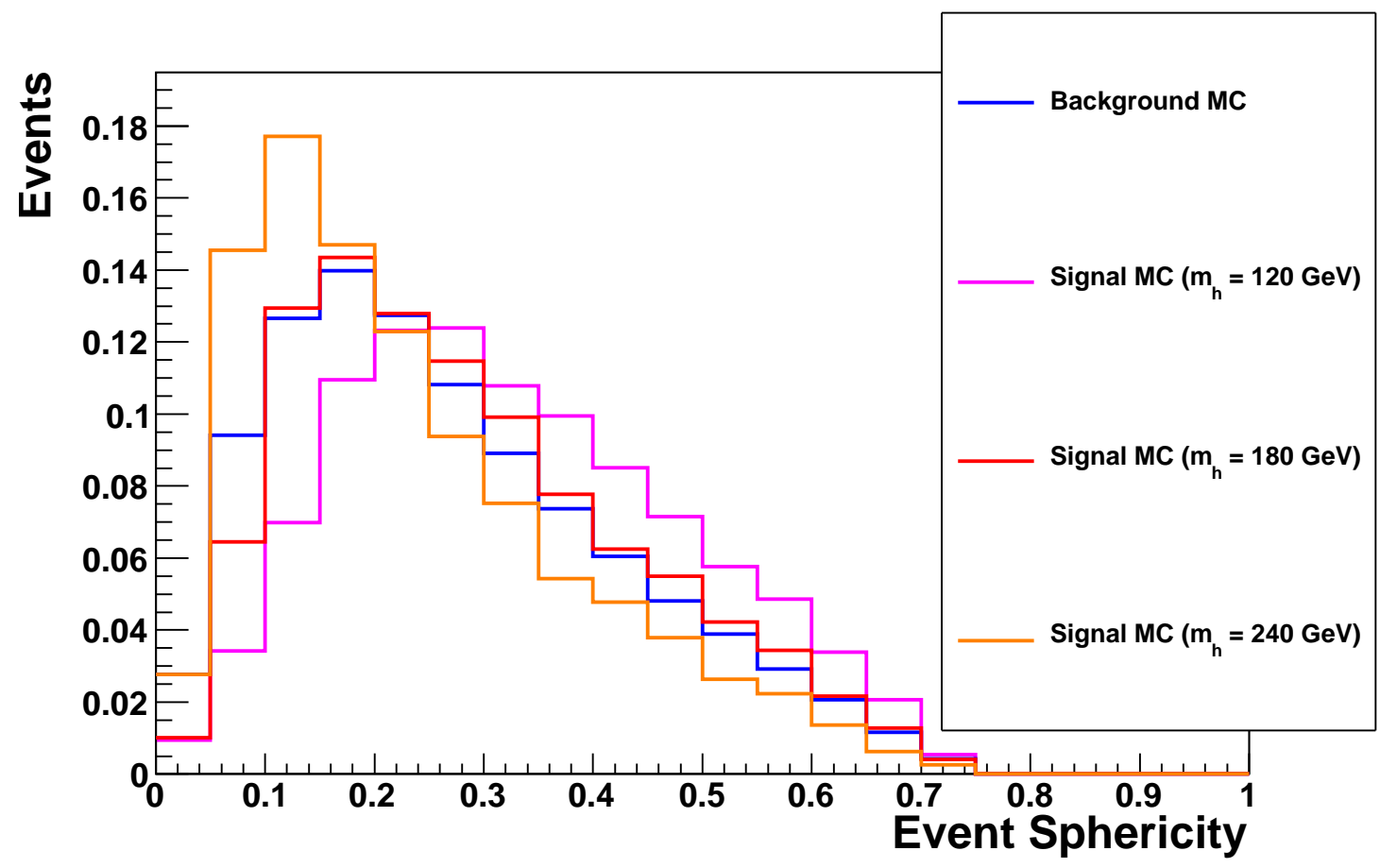

Figure 4.10: The event sphericity distribution for p20 MC in the 3-jet channel. The blue histogram represents $\mathrm{MC}$ background, while the purple, red and orange histograms are signal distributions for $m_{h}$ of 120,180 , and $240 \mathrm{GeV}$ respectively.

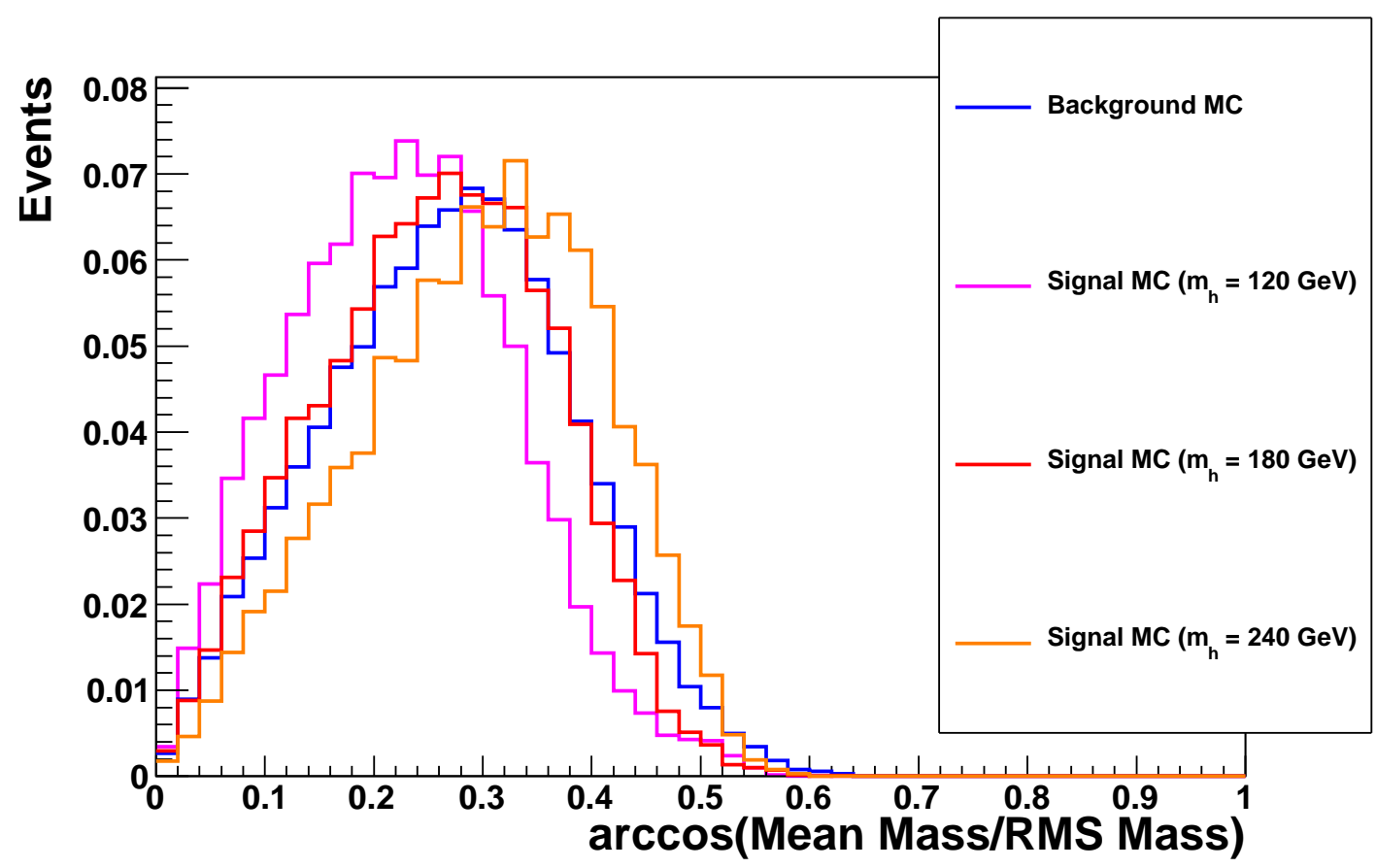

Figure 4.11: The mass deviation angle distribution for p20 MC in the 3-jet channel. This variable is defined in the list of RF input variables. The blue histogram represents MC background, while the purple, red and orange histograms are signal distributions for $m_{h}$ of 120,180 , and $240 \mathrm{GeV}$ respectively. 

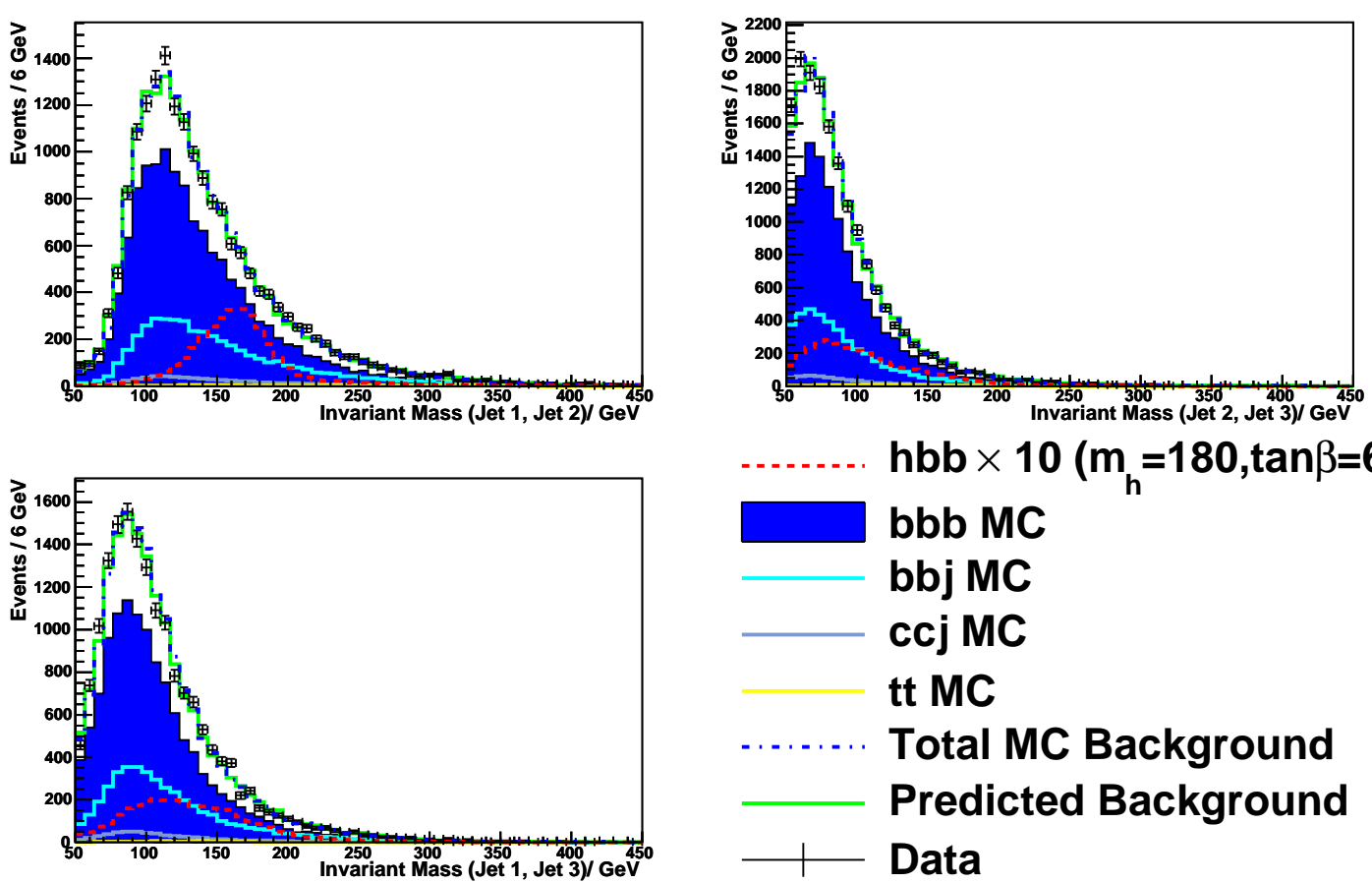

Figure 4.12: The di-jet invariant mass distributions for jet pairs 12 (top left), 23 (top right) and 13 (bottom left) for RunIIb data and MC in the 3-jet channel. The signal histogram is for a Higgs boson with mass $180 \mathrm{GeV}$. 

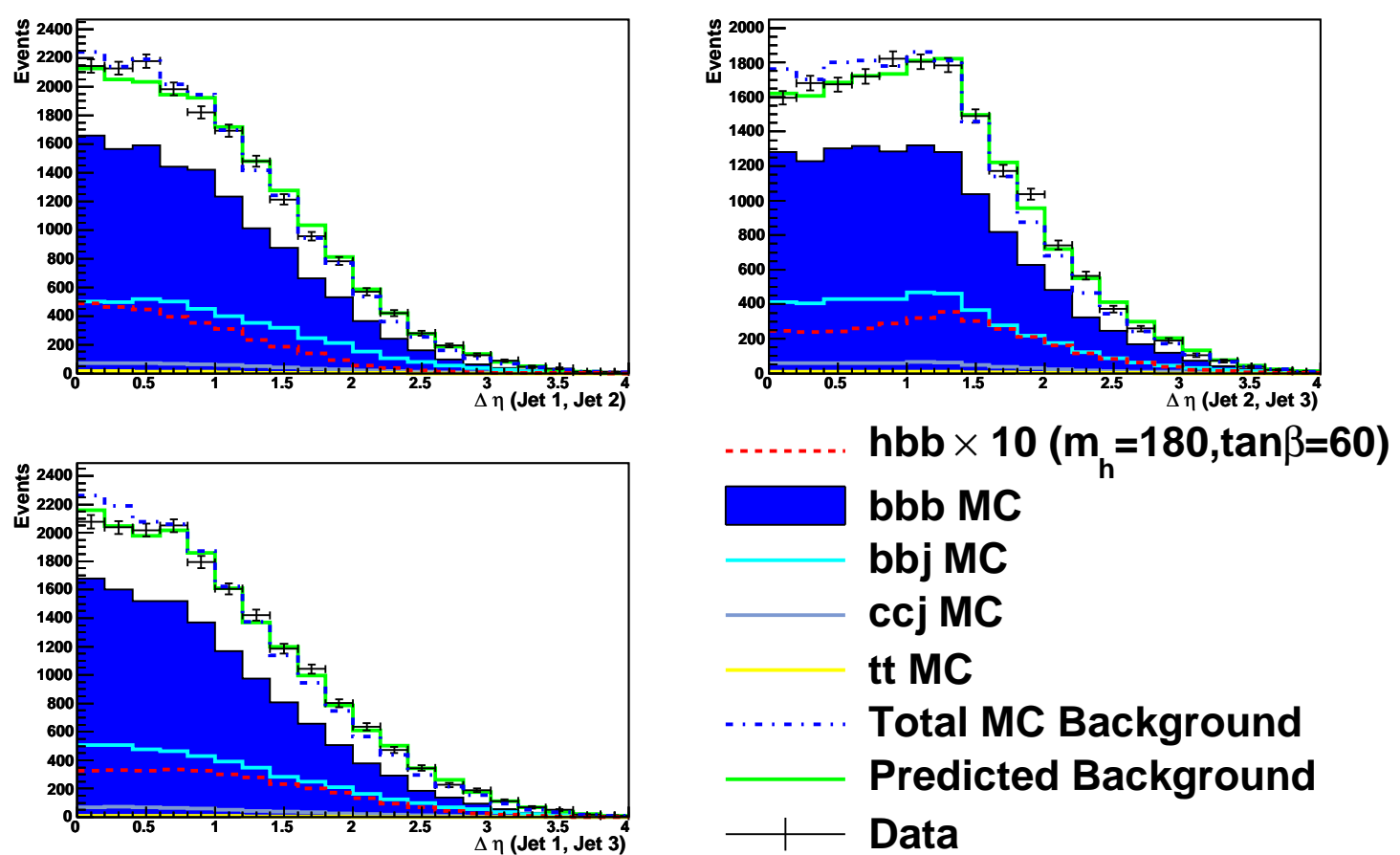

Figure 4.13: The $\Delta \eta$ distributions for jet pairs 12 (top left), 23 (top right) and 13 (bottom left) for RunIIb data and MC in the 3-jet channel. The signal histogram is for a Higgs boson with mass $180 \mathrm{GeV}$.
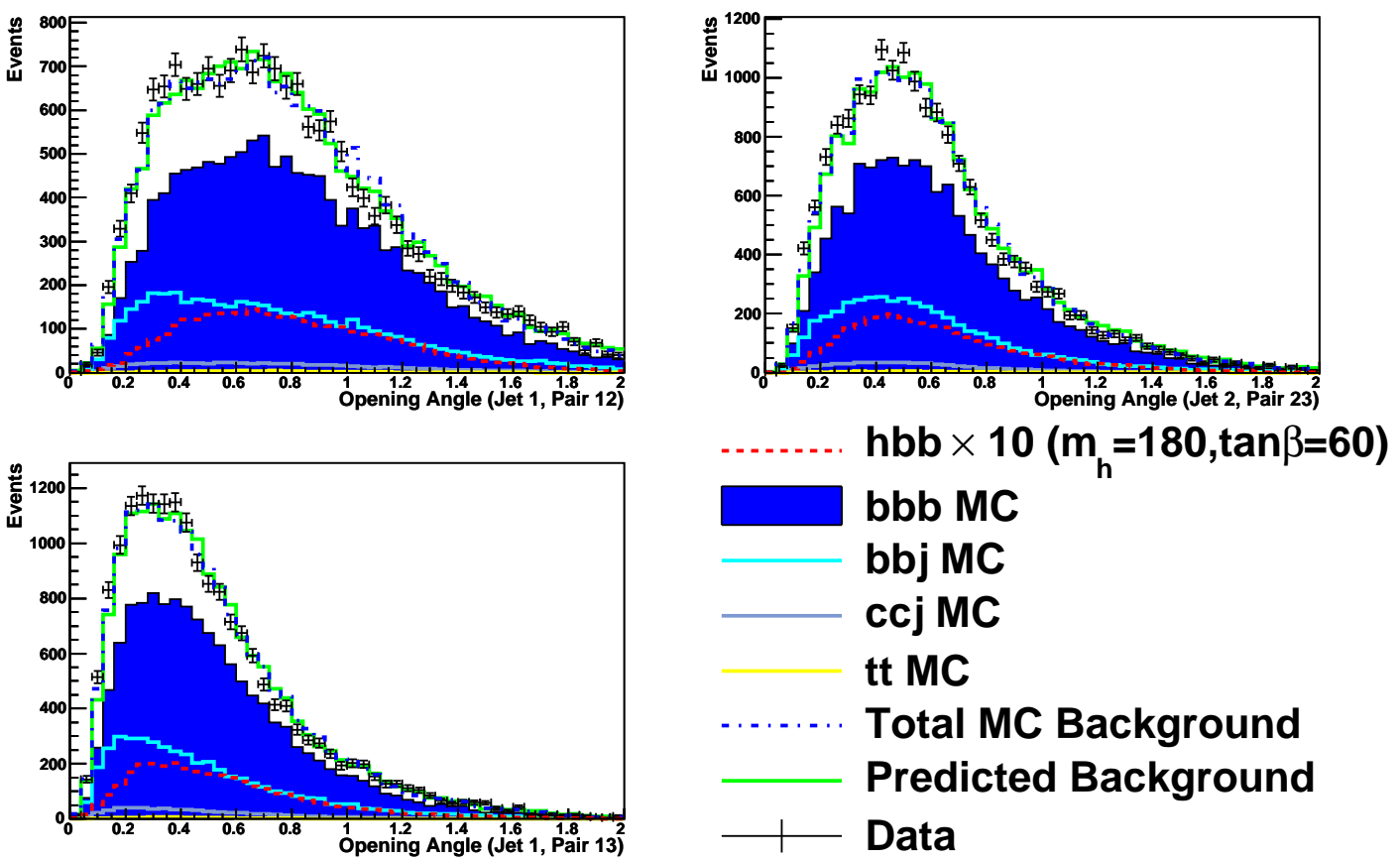

Figure 4.14: The opening angle distributions for jet pairs 12 (top left), 23 (top right) and 13 (bottom left) for RunIIb data and MC in the 3-jet channel. This angle is described in the list of $\mathrm{RF}$ input variables. The signal histogram is for a Higgs boson with mass $180 \mathrm{GeV}$. 


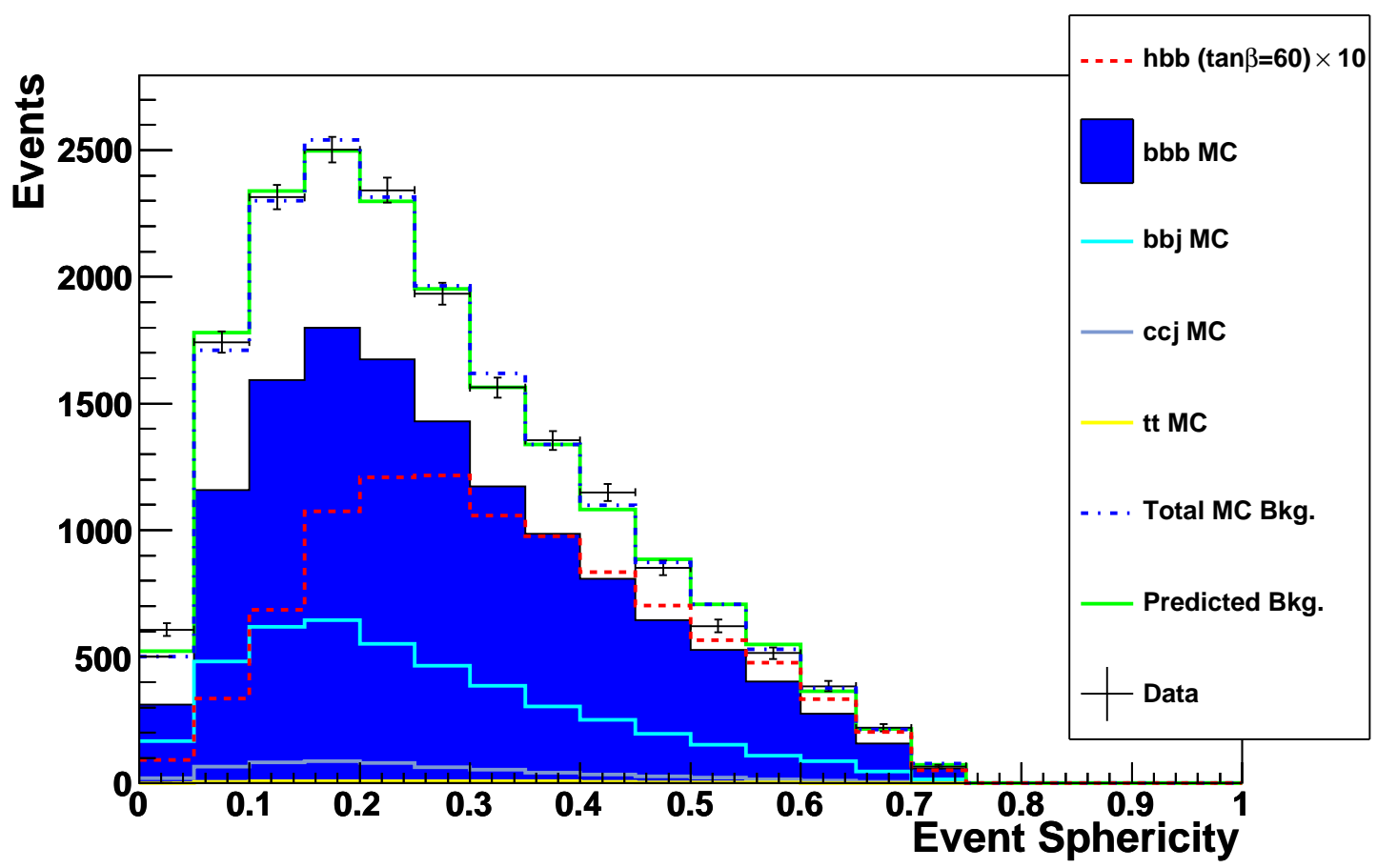

Figure 4.15: The event sphericity distribution for RunIIb data and $\mathrm{MC}$ in the 3-jet channel. The signal histogram is for a Higgs boson with mass $120 \mathrm{GeV}$.

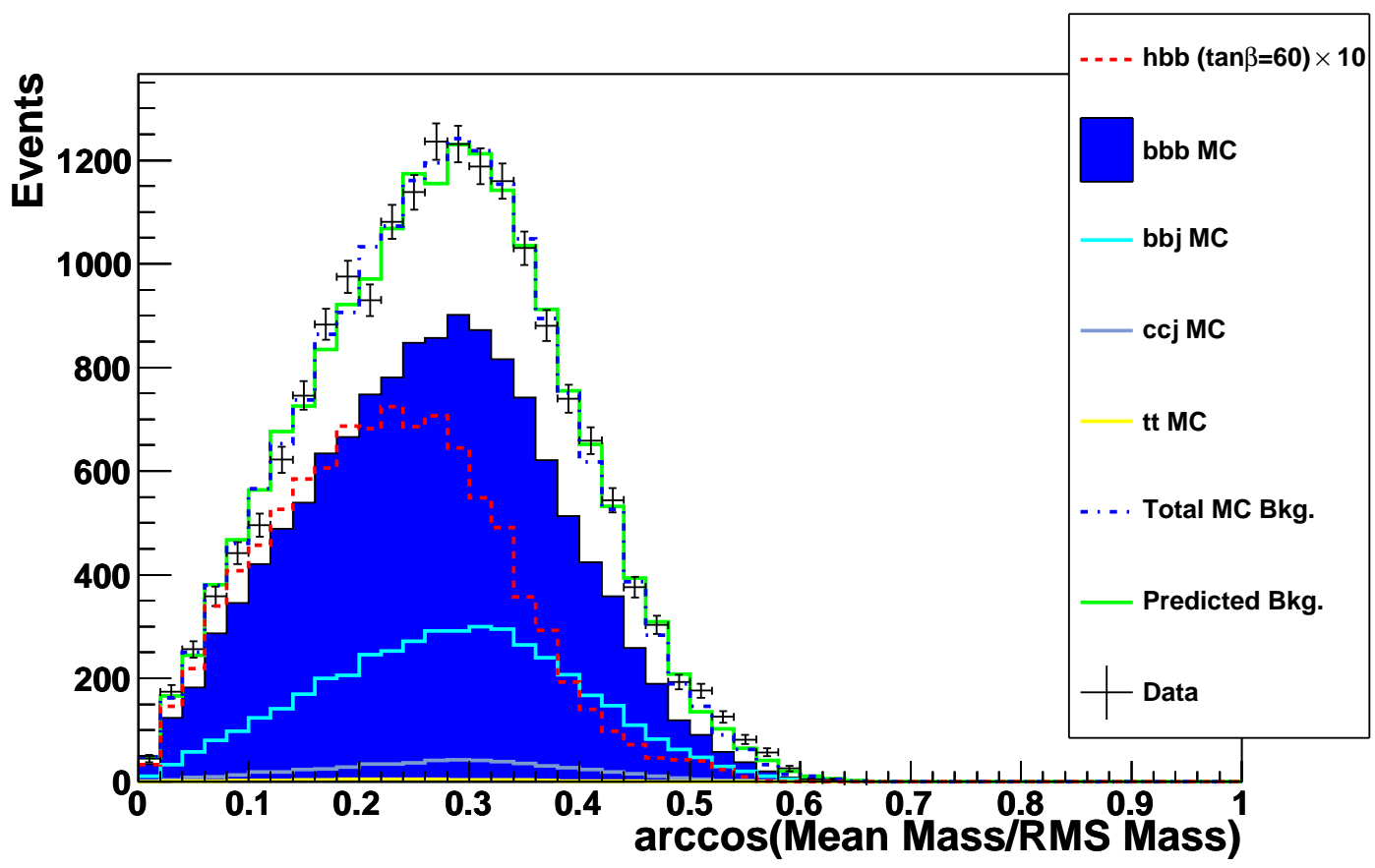

Figure 4.16: The mass deviation angle distribution for RunIIb data and MC in the 3-jet channel. This variable is defined in the list of RF input variables. The signal histogram is for a Higgs boson with mass $120 \mathrm{GeV}$. 
Figures 4.7 to 4.11 show that different variables separate signal and background for different mass hypotheses. For Higgs boson masses above $140 \mathrm{GeV}$ the di-jet invariant masses are the strongest discriminant. For mass hypotheses below 140 $\mathrm{GeV}$, the signal masses lie within the background mass peak, so this variable does not provide good signal-background separation. The event sphericity and deviation angle were included to identify signal events for the low mass hypotheses.

As the di-jet invariant mass was used as a RF input variable, one RF was trained for each of the 24 mass points to maximise sensitivity over the full range of the search. Similarly, the properties of the 3 - and 4 -jet channels were sufficiently different that independent sets of RFs were required for each channel, as well as for the p17 and p20 MC samples. In total $96(24 \times 2 \times 2)$ RFs were trained. The background distribution was different for each RF, so each signal hypothesis was associated with a separate background prediction. Each RF contained 119 DTs: this meant that the RF had 120 discrete output values. The final variable was binned when setting limits, and this number of outcomes allowed several options when binning the discrete variable.

The outputs of the RFs for a Higgs boson of mass $180 \mathrm{GeV}$ are shown for the three- and four-jet channels for RunIIa and RunIIb in Figures 4.17, 4.18, 4.19 and 4.20. These Figures show separation of signal and background events, and that the output for the data and background model are in reasonable agreement. The background RF output distribution was obtained from the RF output distributions for the 3-tag and 2-tag MC samples and the 2-tag Data samples, as described in Section 3.2.6.

In general, these RF distributions show good data-background agreement and good signal-background separation. 


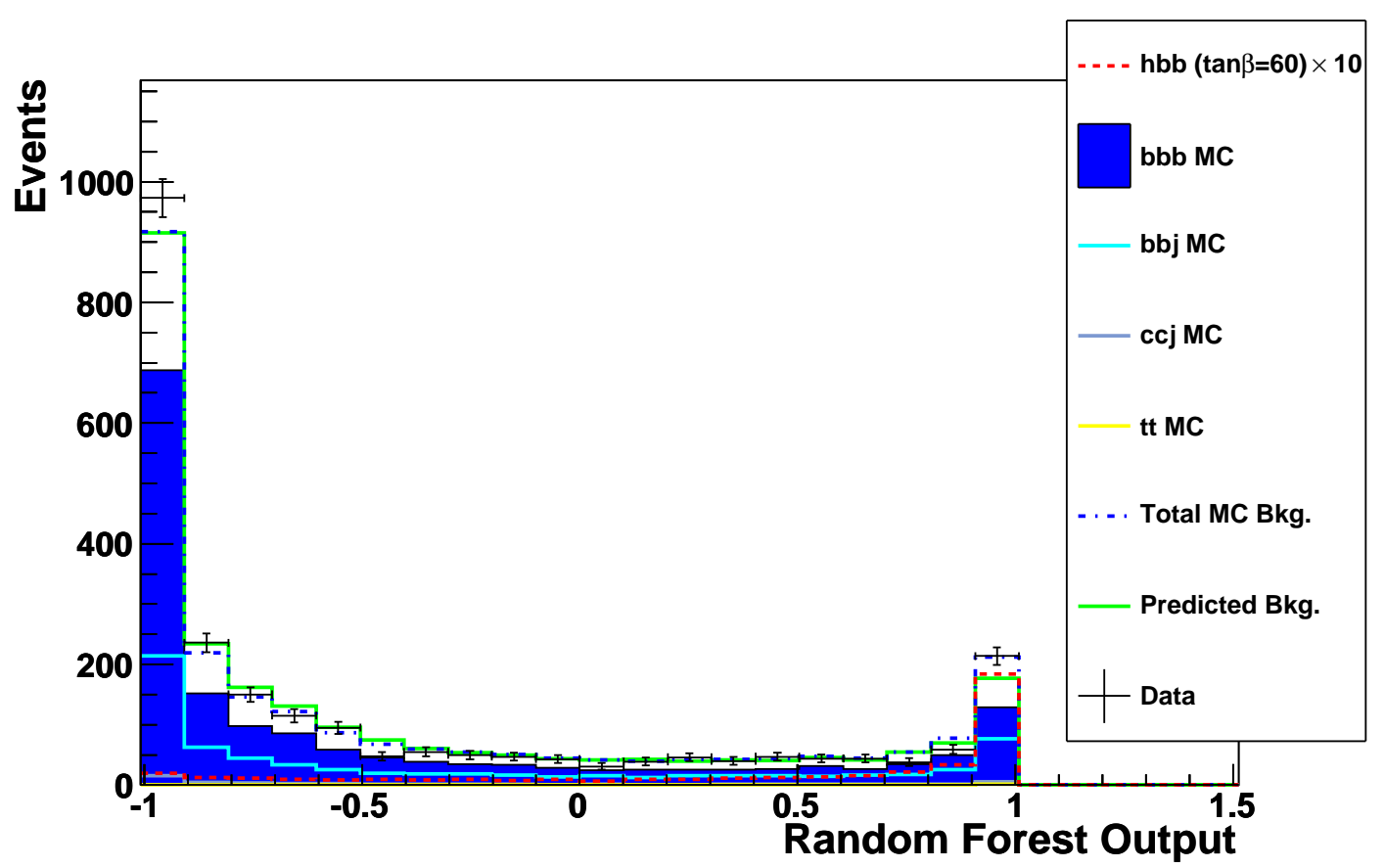

Figure 4.17: The random forest output distribution for a Higgs boson mass of $180 \mathrm{GeV}$ in the 3 -jet channel (RunIIa).

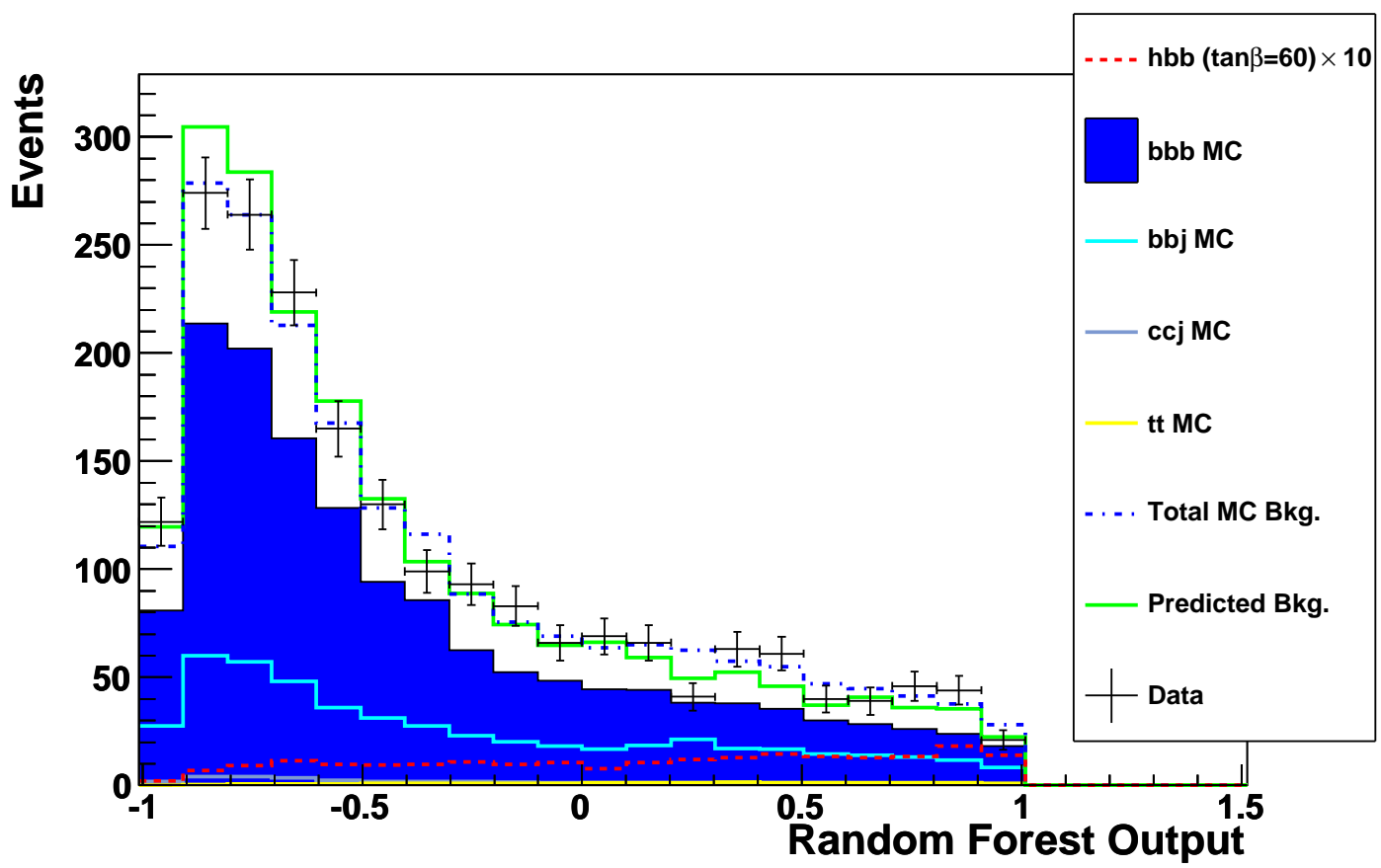

Figure 4.18: The random forest output distribution for a Higgs boson mass of $180 \mathrm{GeV}$ in the 4 -jet channel (RunIIa). 


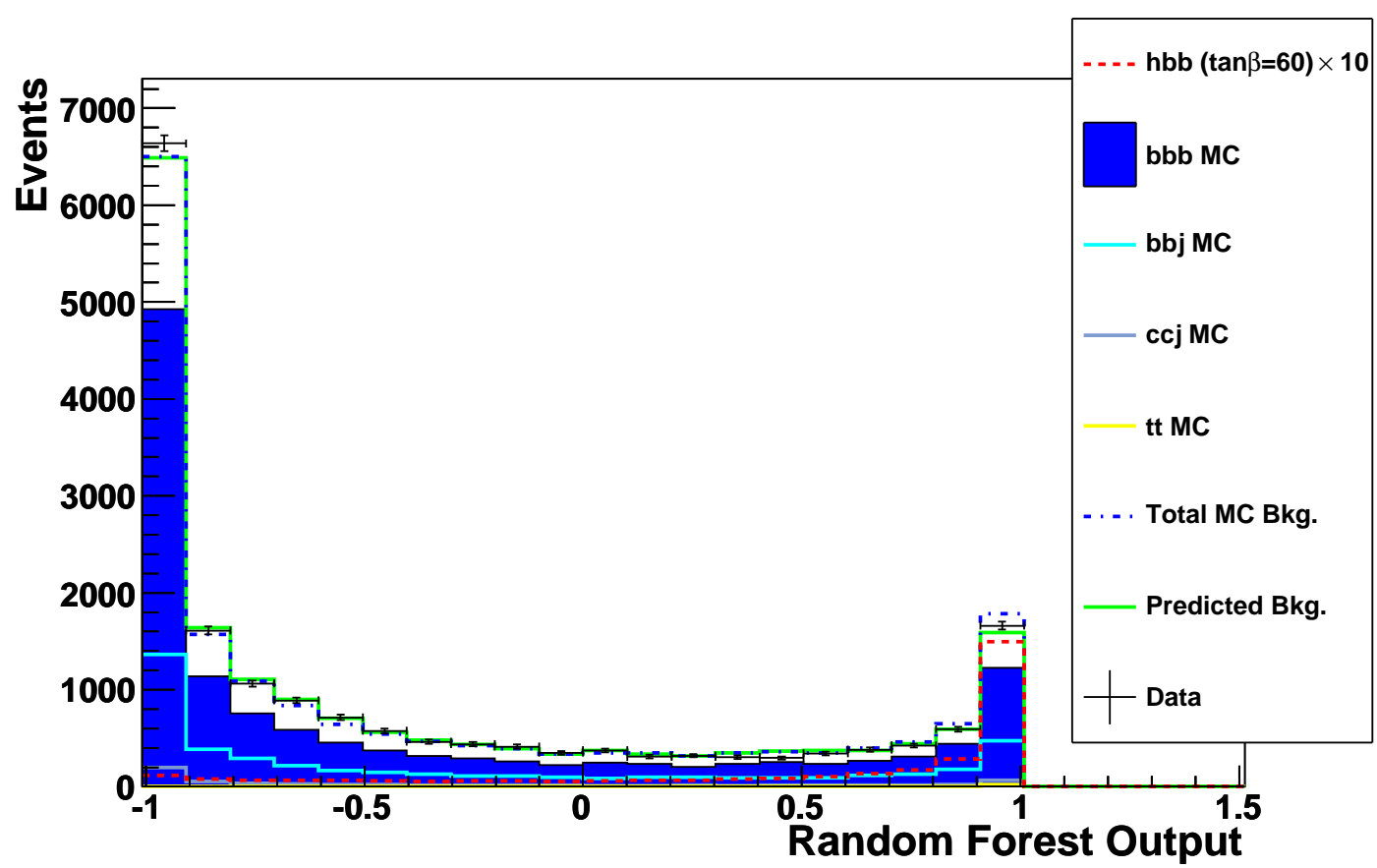

Figure 4.19: The random forest output distribution for a Higgs boson mass of $180 \mathrm{GeV}$ in the 3 -jet channel (RunIIb).

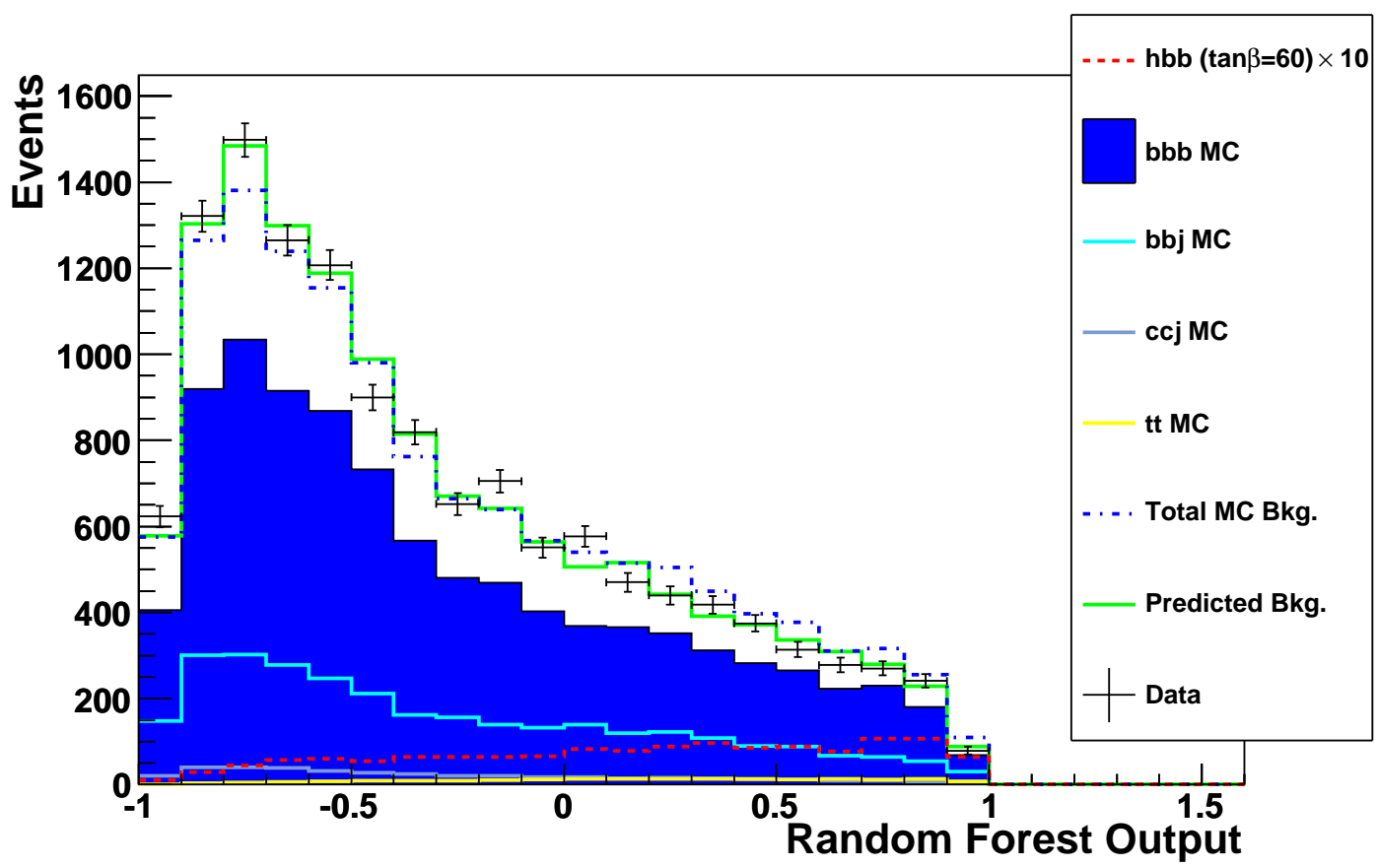

Figure 4.20: The random forest output distribution for a Higgs boson mass of $180 \mathrm{GeV}$ in the 4 -jet channel (RunIIb). 


\subsection{Conclusion}

Three different MVA techniques were considered for use in the $\phi b \rightarrow b \bar{b} b$ analysis. Of these methods, the Random Forest of Decision Trees method was found to give the best stability, and displayed good signal-background discrimination, so the RF method was chosen to provide the final variable in the analysis. A set of input variables was chosen for the RF that allowed strong separation of signal and background, as well as having good data-background agreement. 


\section{Chapter 5}

\section{Confidence Limits on $\sigma \times B R$ for the Process $\phi b \rightarrow b b b$}

\section{$5.1 \quad$ Introduction}

This chapter describes the method used to set confidence limits on the value of $\sigma \times B R$ for the $\phi b \rightarrow b \bar{b} b$ process, and by extension to exclude a region of the $\left(m_{A}, \tan \beta\right)$ parameter space for the MSSM. This chapter also includes a discussion of the sources of systematic uncertainty considered for inclusion in the limit setting procedure.

\subsection{Systematic Uncertainties}

The systematic uncertainties for the analysis were estimated by altering various properties of the MC samples, and propagating these effects through to the final limit setting observable. In the limit setting process, both the scale and the shape of a systematic uncertainty affects the limit setting, so both must be evaluated. For the signal hypothesis, the effect of the uncertainties could be investigated by varying the relevant parameter to derive an alternative signal Random Forest (RF) distribution.

In the case of the background, varied RF distributions for the 3- and 2-tag MC background were generated, and the 2-tag data RF distribution was multiplied by the modified ratio. The background normalisation was unknown, so the normalisation was treated as an extra source of uncertainty. The normalisation of the different uncertainties was allowed to float in the limit setting process, so only the shape component of each background uncertainty was considered. This was achieved by normalising each varied background RF distribution to equal area with the nominal background distribution. 


\subsubsection{Systematic Uncertainties on the Signal}

The following sources of uncertainty on the signal were investigated:

- Heavy-jet TRF: the b-tagging efficiencies for MC b- and c-jets were varied within $\pm 1 \sigma$ to give an alternative weight for each event. The efficiencies (and their standard deviations) varied with $H_{T}$, so the efficiency was varied according to the event $H_{T}$. These were used to construct an alternative signal RF distribution.

- Heavy- and light-jet shape: the shapes of the heavy jet b-tagging TRFs with respect to $p_{T}$ were used in the determination of the light jet TRF (or fake rate). The impact of the uncertainty on the fake rate on the final discriminant was investigated by changing the shape templates for the TRFs and re-weighting the signal MC accordingly. The shape templates are derived from data and $\mathrm{MC}$, and so both templates were altered to evaluate this systematic. The uncertainty due to these quantities for the signal sample was found to be negligible. This was expected, as the light jet contribution to the simulated $b b b$ sample is minimal. As a result, this systematic was not included in the limit setting.

- Trigger: the effect of the uncertainty on the trigger efficiency on the signal simulation was investigated by altering the efficiencies of b-tagging and kinematic triggers within errors [84].

- JES: corrections to the jet energies are derived from measurements of energy imbalances in photon-jet events (see [69] for details). This correction is applied according to the uncorrected $E_{T}$ and $\eta$ of the jet. Uncertainties are associated with these correction factors, so the energy correction was varied by $\pm 1 \sigma$ for each jet. The event weights were dependent on jet $p_{T}$, and so were re-calculated accordingly. Varying the energy of the jets caused the di-jet invariant masses to change: this led to large bin-to-bin fluctuations in the RF distribution. The method used to deal with these fluctuation is outlined below.

- JSSR: in a similar manner to the jet energy scale, the jet energy resolution and identification efficiency were corrected by investigating photon-jet events [70]. Again, there were uncertainties in these corrections, so the resolution was varied within $\pm 1 \sigma$, and event weights were re-calculated for the new jet $p_{T}$ values to form an alternative signal distribution. Again, this uncertainty displayed large bin-to-bin fluctuations. 
- b-jet resolution: the possibility exists that the energy resolution of the b-jets was not modelled as well as the energy scale of the light jets, so the b-jet energy was smeared by an additional $7 \%$ in the signal MC (the maximum energy uncertainty for light jets is $15 \%$ : a smearing of an additional 0.5 of this value was used as a conservative estimate of the maximum uncertainty). This uncertainty caused bin-to-bin fluctuations in a similar fashion to the JSSR uncertainty.

- Taggability: two alternative taggability scale factors, (derived for negative and positive values of $\left.\eta_{\text {jet }} \times z_{P V j e t}\right)$ were used to estimate the uncertainty due to taggability. This uncertainty was found to be less than $1 \%$, so this uncertainty was not considered in the limit setting.

- Luminosity: the standard DØ luminosity error prediction, a flat uncertainty of $6.1 \%$ [83], was applied to the signal MC.

- Theoretical uncertainty: there were two sources of uncertainty related to the theoretical predictions used in the signal simulation. The first source of uncertainty is the set of twenty empirically-derived CTEQ parton density functions [74] used to predict the signal. The uncertainty on these parameters contributes to an uncertainty on the cross section. This was estimated by forming a set of orthogonal eigenvectors, two (positive and negative) for each parameter, and fluctuating each parameter within $\pm 1 \sigma$. The total uncertainty was calculated by adding the positive and negative fluctuated values in quadrature. The signal cross section was also affected by the renormalisation and factorisation scales used in the MCFM simulation [73]. Both scales were varied by factors of 0.5 and 2 , and the resulting changes propagated through to the cross section calculation to give the uncertainty. The MCFM and CTEQ uncertainties were added in quadrature to give a single theoretical systematic uncertainty.

The b-jet resolution, JES and JSSR uncertainties varied the energy and momentum of the b-jets, thus varying the RF output for these events. These resolution systematic uncertainties displayed large bin-to-bin fluctuations, so polynomials were fitted to the residuals to determine the underlying shape uncertainty (although the nominal and systematically altered distributions had a peaked distribution in the final discriminant, the residuals had relatively smooth shapes so polynomials could be fitted to them). Example fits are shown in Figures 5.1 and 5.2. The method used 


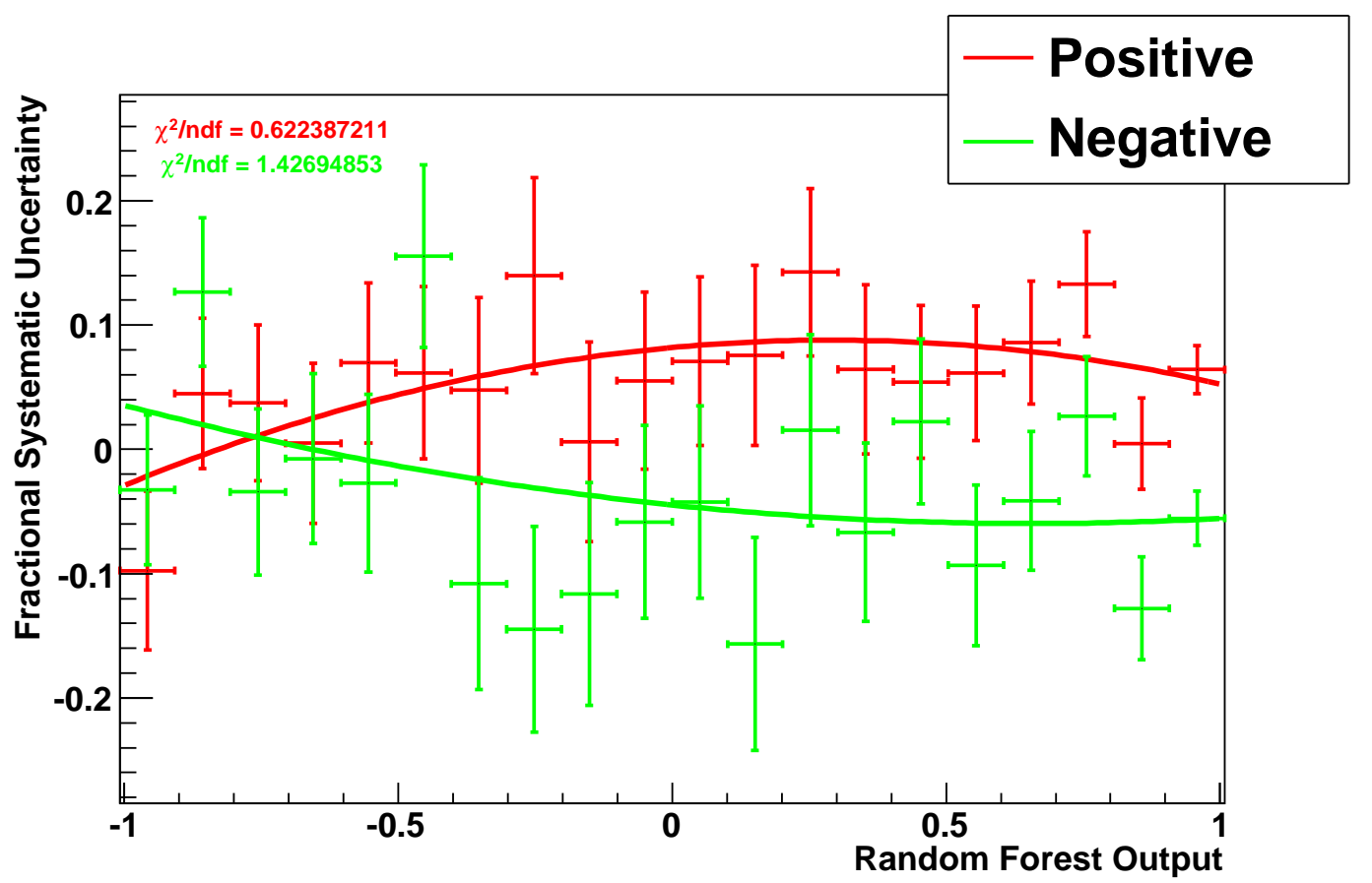

Figure 5.1: Systematic uncertainties on the signal due to the jet energy scale for the three jet channel in RunIIb data, plotted as a fraction of the nominal signal distribution (for a Higgs boson mass of $180 \mathrm{GeV}$ ). A second order polynomial was fitted to the positive and negative fluctuations.

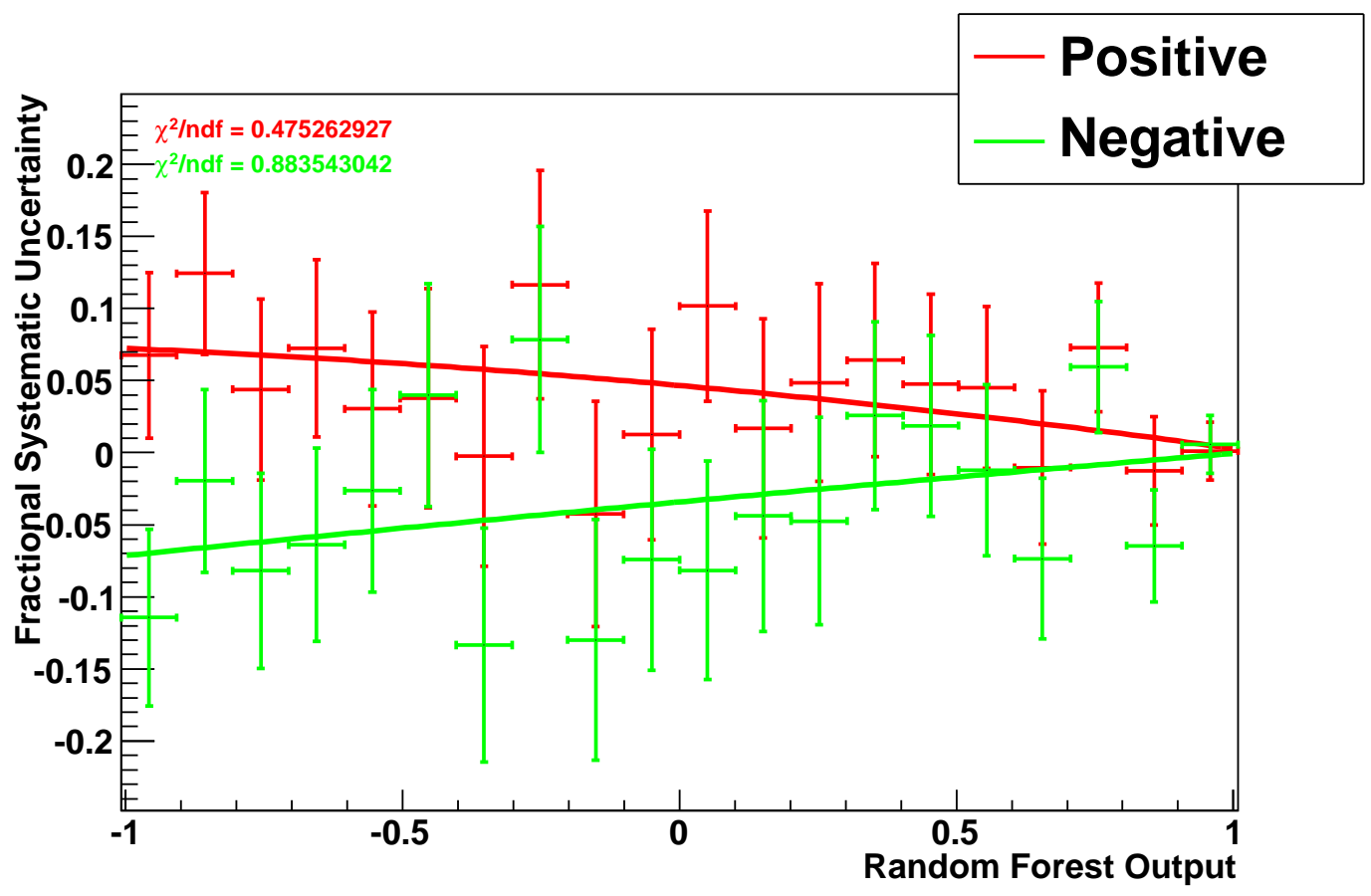

Figure 5.2: Systematic uncertainties on the signal due to the JSSR for the three jet channel in RunIIb data, plotted as a fraction of the nominal signal distribution (for a Higgs boson mass of $180 \mathrm{GeV}$ ). A second order polynomial was fitted to the positive and negative fluctuations. 
to incorporate systematic errors in the limit setting program relied on the positive and negative fluctuations being symmetric, so the fit with the largest deviation was selected and used for both fluctuations to avoid complications.

The signal systematic uncertainties for a mass hypothesis of $180 \mathrm{GeV}$ in the four channels are plotted in Figure 5.3 to 5.6. In these plots only one fluctuation (positive or negative) is shown: most of the systematic uncertainties were symmetric, but in the case of a difference in scale, the larger fluctuation is plotted. To prevent large shape uncertainties due to low statistics in individual bins, a smoothing algorithm in the limit setting program merged bins to give roughly equal numbers of events per bin [85] (note that this algorithm distorts the shape of the fitted polynomials in Figures 5.1 and 5.2).

The largest source of uncertainty, in terms of scale, was found to be the b-jet TRF; the b-tagging was expected to be the dominant source of error.

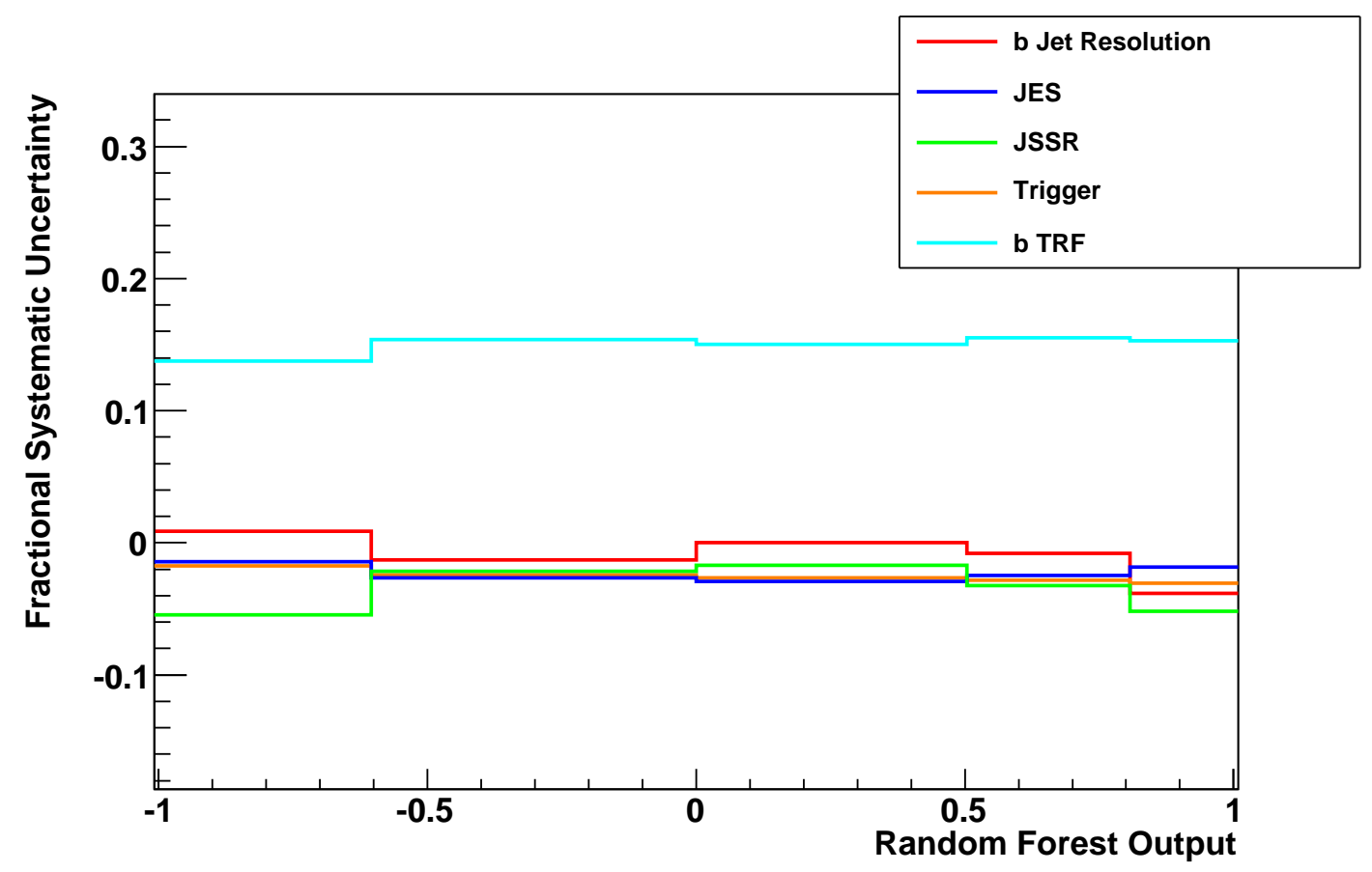

Figure 5.3: Systematic uncertainties on the signal, plotted as a fraction of the signal distribution as a function of the p17 three jet channel RF output for a Higgs boson mass of $180 \mathrm{GeV}$.

\subsubsection{Systematic Uncertainties on the Background}

The following sources of uncertainty were evaluated for the background:

- Heavy-jet TRF: the b-tag efficiencies for MC b- and c-jets were varied within $\pm 1 \sigma$ to give an alternative weight for each event. The TRF also affected the 


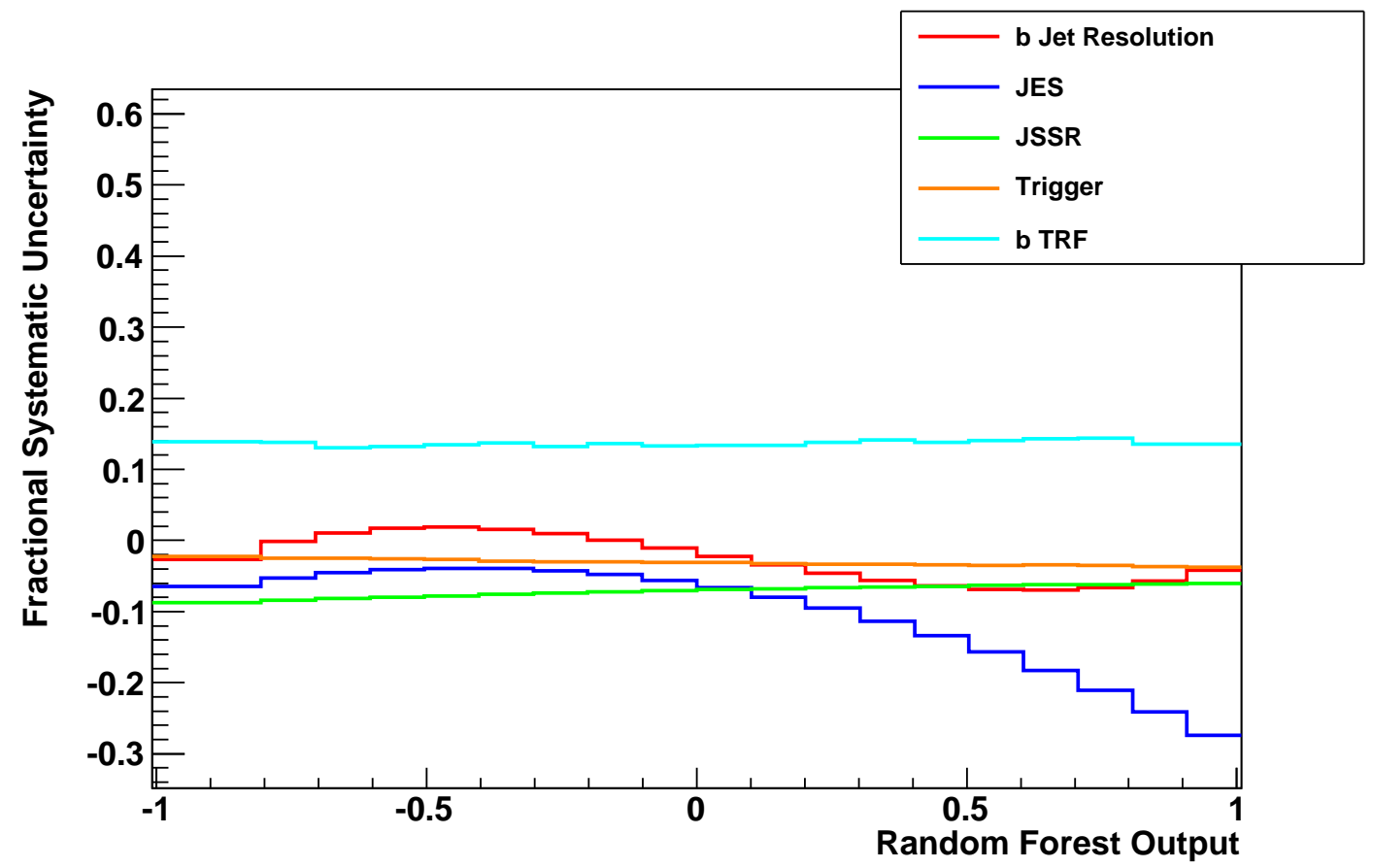

Figure 5.4: Systematic uncertainties on the signal, plotted as a fraction of the signal distribution as a function of the p17 four jet channel RF output for a Higgs boson mass of $180 \mathrm{GeV}$.

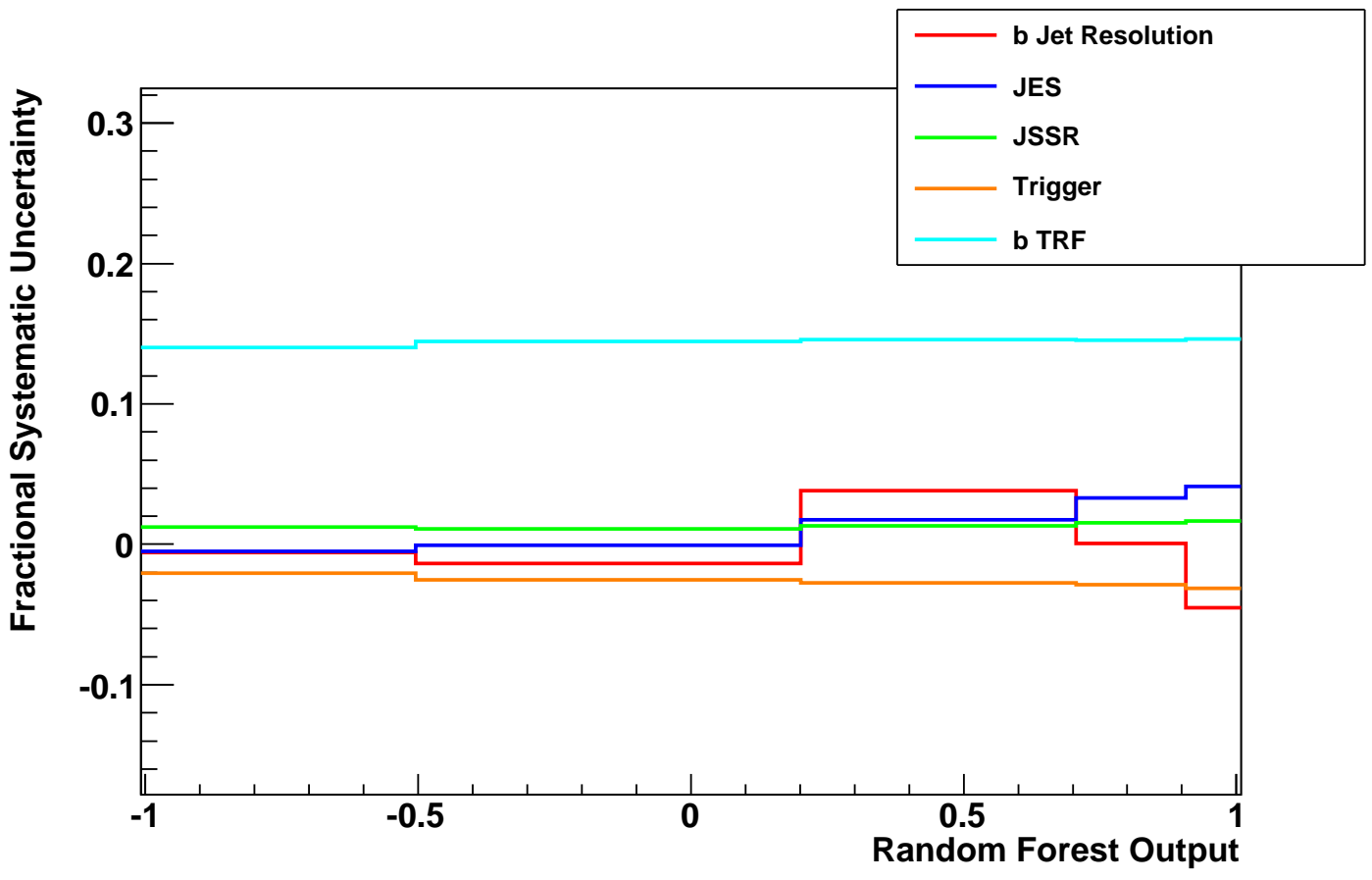

Figure 5.5: Systematic uncertainties on the signal, plotted as a fraction of the signal distribution as a function of the p20 three jet channel RF output for a Higgs boson mass of $180 \mathrm{GeV}$. 


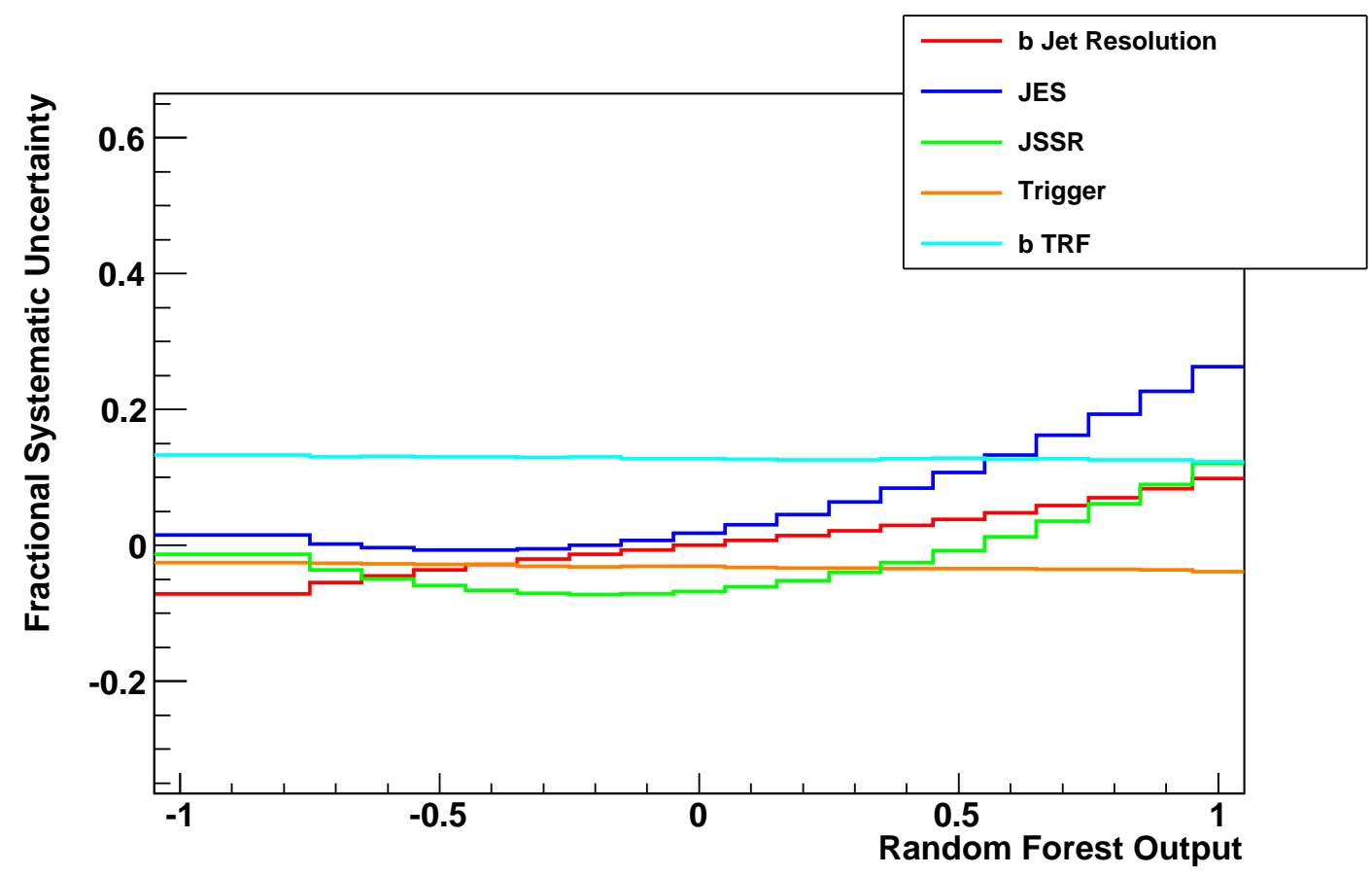

Figure 5.6: Systematic uncertainties on the signal, plotted as a fraction of the signal distribution as a function of the p20 four jet channel RF output for a Higgs boson mass of $180 \mathrm{GeV}$.

background composition, so this effect was propagated through to the event weights. Alternative 3-tag and 2-tag MC distributions were generated, and these were used to form an alternative background model. Before normalisation, the positive and negative uncertainties were symmetric within $\pm 1 \%$. After normalisation, however, the overall scale of the uncertainty was roughly $1 \%$, which led to an exagerated asymmetry. The shapes of these uncertainties were the same, so the fluctuation with the largest scale was used for both positive and negative variations.

- Heavy- and light-jet shape: the shapes of the di-jet invariant mass distributions were used in the determination of the fake rate. The impact of the uncertainty on the fake rate on the final discriminant was investigated by changing these shape templates and recalculating the fake rate. The shape templates are derived from data and $\mathrm{MC}$, and so both templates were altered to evaluate this uncertainty.

- Trigger: it is possible that the b-tagging triggers have different efficiencies for 2-tag and 3-tag events. The affect of this uncertainty on the background was ascertained using data collected using an additional trigger [84]. This trigger had no L3 requirements, but the L1 and L2 triggers were the same as those 
used in the analysis. The events collected by this trigger were analysed using a simulation of the L3 trigger, with a 3 -tag requirement and a 2-tag requirement. These samples were plotted against di-jet invariant mass of the leading pair, and a polynomial was fitted to the ratio. This polynomial was used to reweight the analysis 3-tag to 2-tag ratio, forming a new background model. Several trigger lists were used in different runs, so the efficiency distributions for these were calculated separately, and a luminosity averaged sum of the resulting fit polynomials was used to adjust the background model. Varying the trigger efficiencies in this way caused the background normalisation to change; however, the variation was largely independent of the invariant mass, so the shape change due to this effect was negligible. For this reason, the systematic uncertainty on the background due to the trigger was not taken forward to the limit setting process.

- JES: the jet energy scale (JES) was varied within $\pm 1 \sigma$ for the 3 -tag and 2tag MC background samples in a similar manner to the signal sample. An alternative background model was derived from these samples. Similarly to the signal JES uncertainty, this caused the RF output values to change, so the fractional uncertainty distribution showed bin to bin fluctuations. In a similar manner to the signal JES error, a polynomial was fitted to the shape of the uncertainty. The change in background scale, however, cancelled in the 3-tag to 2-tag ratio, so the uncertainty due to JES was found to be consistent with a flat uncertainty at $0 \%$, as shown in Figure 5.7.

- JSSR: the JSSR for the MC background was varied within $\pm 1 \sigma$ in a similar manner to the JSSR systematic uncertainty on the signal. Once the 3-tag to 2-tag ratio was taken, this was consistent with a flat systematic uncertainty of $0 \%$, illustrated in Figure 5.7.

- b-jet resolution: the jet energy of b- and c-jets was smeared by an additional $7 \%$ in the 3-tag and 2-tag MC distributions to construct an alternative background prediction. This systematic showed bin-to-bin fluctuations, but the scale was similar for 3- and 2-tag events, so the normalisation cancelled in the 3 -tag to 2 -tag ratio. The remaining systematic uncertainty showed little shape variation and was close to $0 \%$, so this systematic was not included in the limit setting. 
- Signal contamination: because the background composition is found by fitting to data, any signal in this data sample would cause an error in the bbb contribution. The effect of the presence of signal was estimated by increasing the bbb contribution to the 3-tag MC consistently with the presence of signal (with a $\tan \beta$ that had previously been excluded), and re-deriving the background model.

- Top background: $t \bar{t}$ events in data are far less common than the light-jet multijet events that make up the majority of the background. However, it is possible that top events are identified as signal events by the RF, leading to a bias in the final discriminant. The top contribution was increased by $10 \%$ [60] to determine how much this affected the background distribution. The top jet contribution to the four jet background was larger than that for the three jet background (as shown in the plots in Section 4.5) so the uncertainty due to the top cross section was larger in the four jet channel.

The fractional change in the RF output distribution due to each of these effects is shown in Figures 5.8 to 5.11. The systematic uncertainties are shown for the alternative background models before and after the RF distributions were normalised to equal area with the nominal background RF distribution.

Because one RF was trained for each signal mass hypothesis, a corresponding set of alternative background RF distributions (one for each systematic) was included for each signal hypothesis.

The dominant source of uncertainty on the background was the b-tagging; this was to be expected as the background model was reliant on the number of tight tagged jets in the data and MC samples. The shape variation of these fractional uncertainties was quite small, in some cases less than $1 \%$. This implies that the 3-tag to 2-tag MC ratio as a function of the RF output was insensitive to systematic changes. 


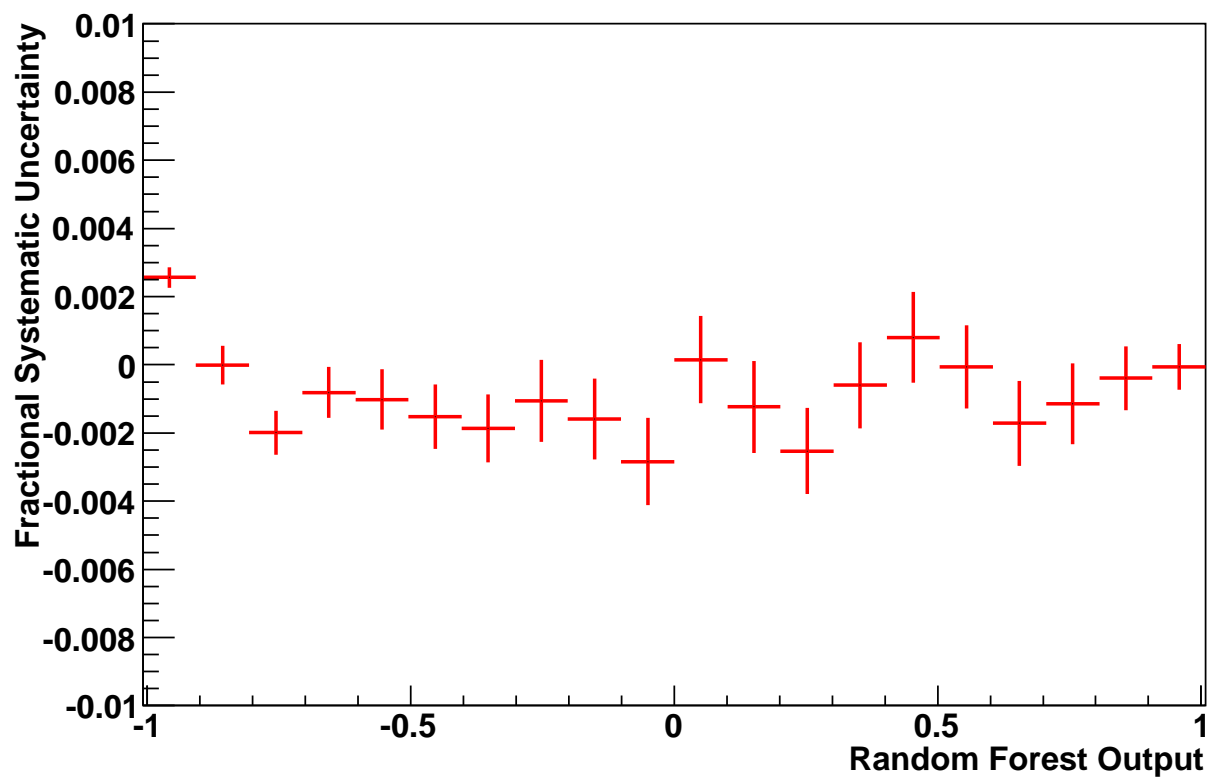

(a)

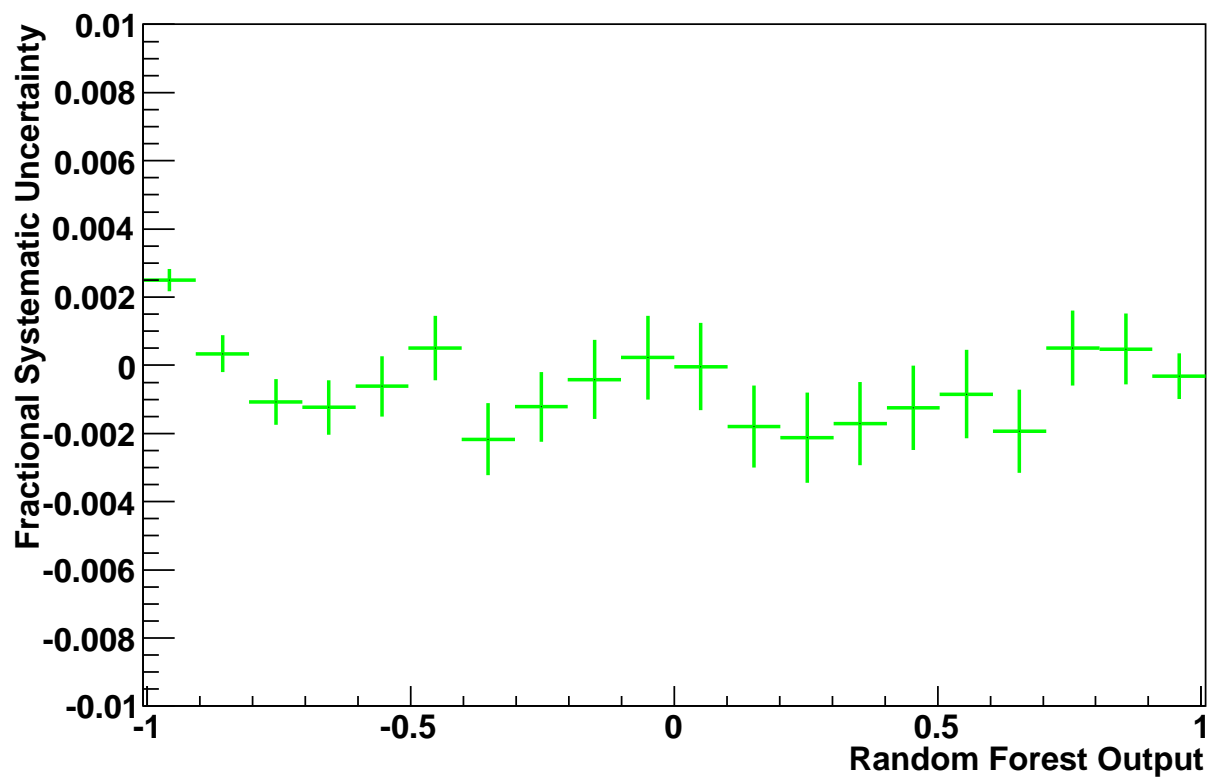

(b)

Figure 5.7: Systematic uncertainties as a fraction of the nominal predicted background for positive fluctuations of (a) JES and (b) JSSR. When fitting a polynomial to these systematics, it was found that they were consistent with a flat systematic at 0 . 


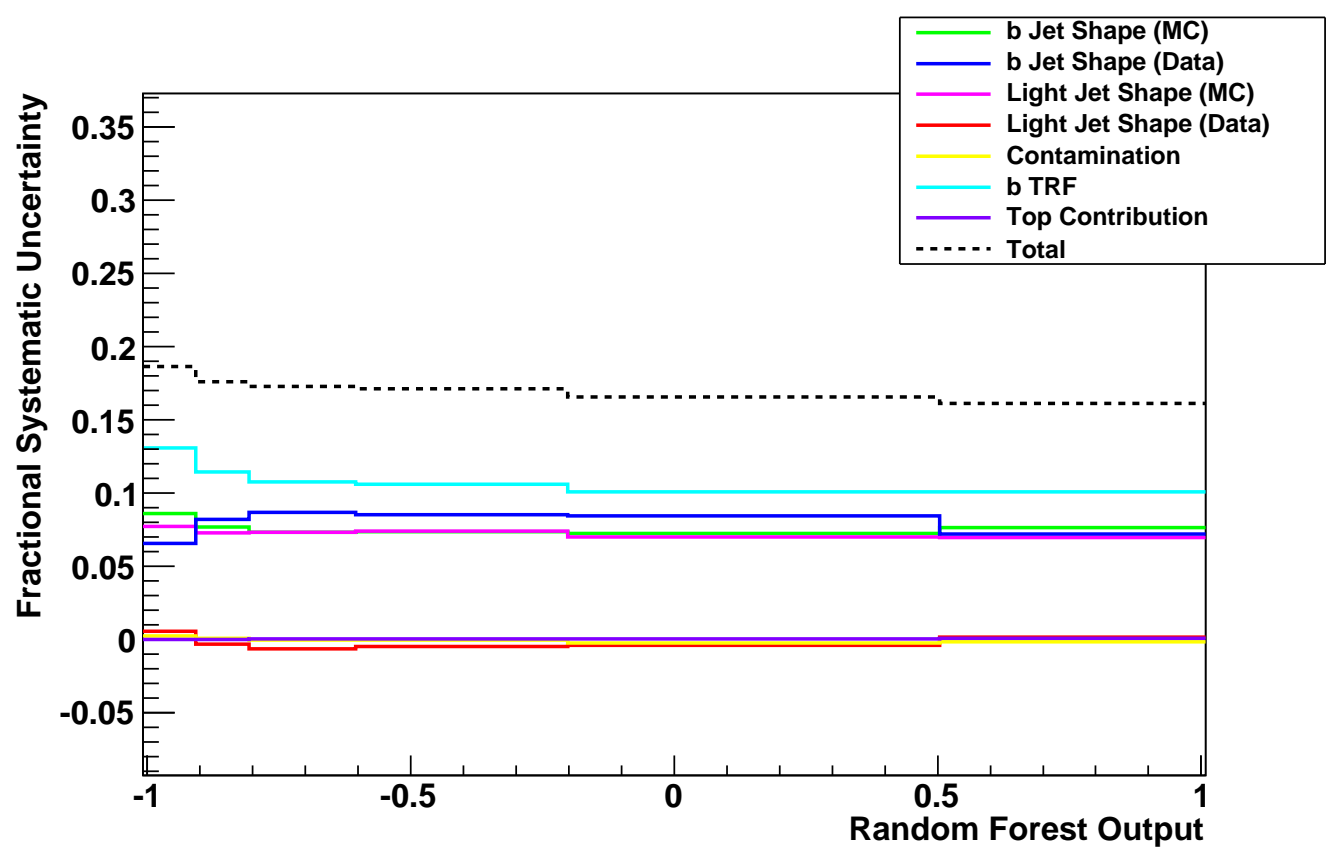

(a)

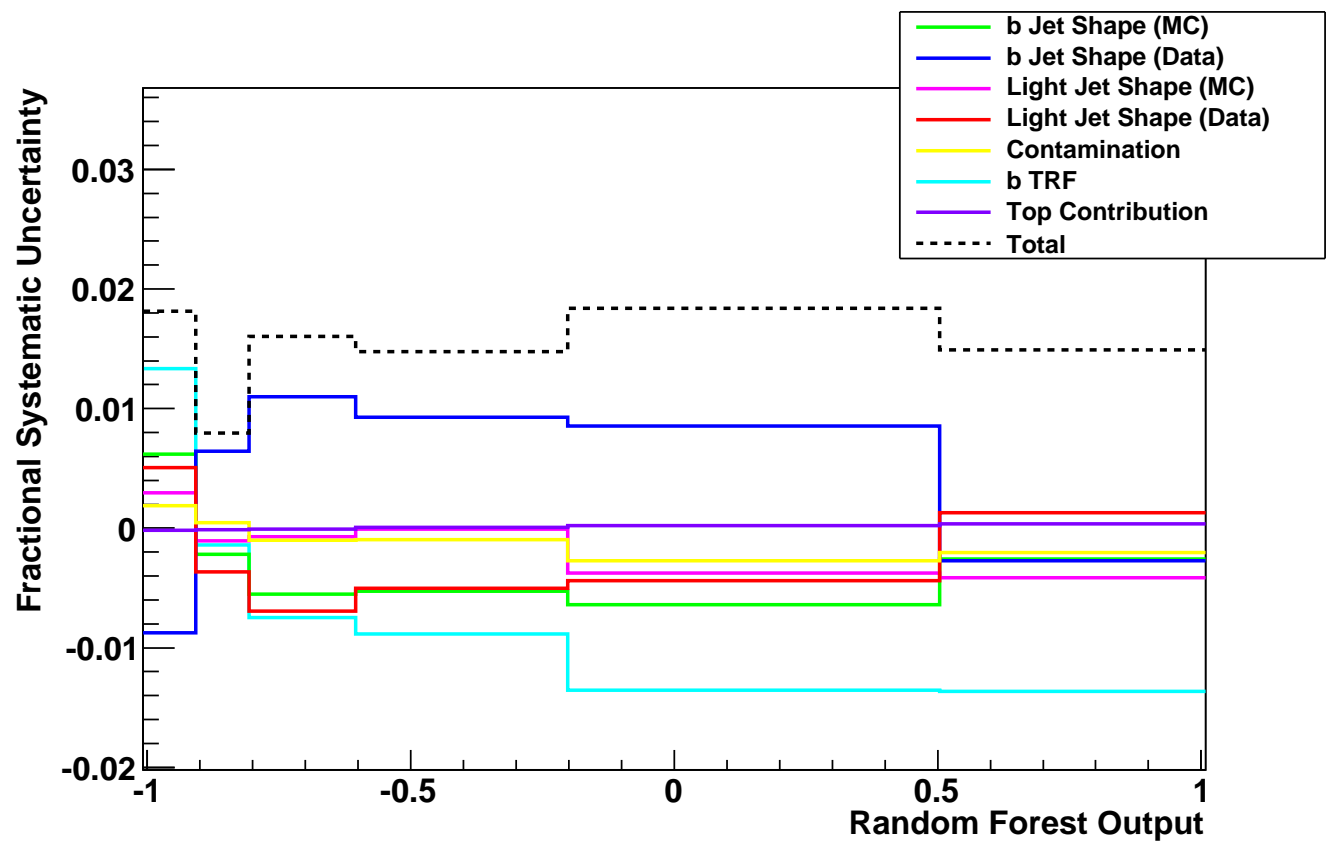

(b)

Figure 5.8: Systematic uncertainties as a fraction of the nominal predicted background plotted as a function of the p17 three jet channel RF output for a Higgs mass of $180 \mathrm{GeV}$. The systematics are shown both (a) un-normalised and (b) normalised to equal area with the predicted background. The black dotted line in (b) is intended to give an indication of the total uncertainty: this is not the true total because the scale of the nominal background is allowed to float in the limit setting process. 


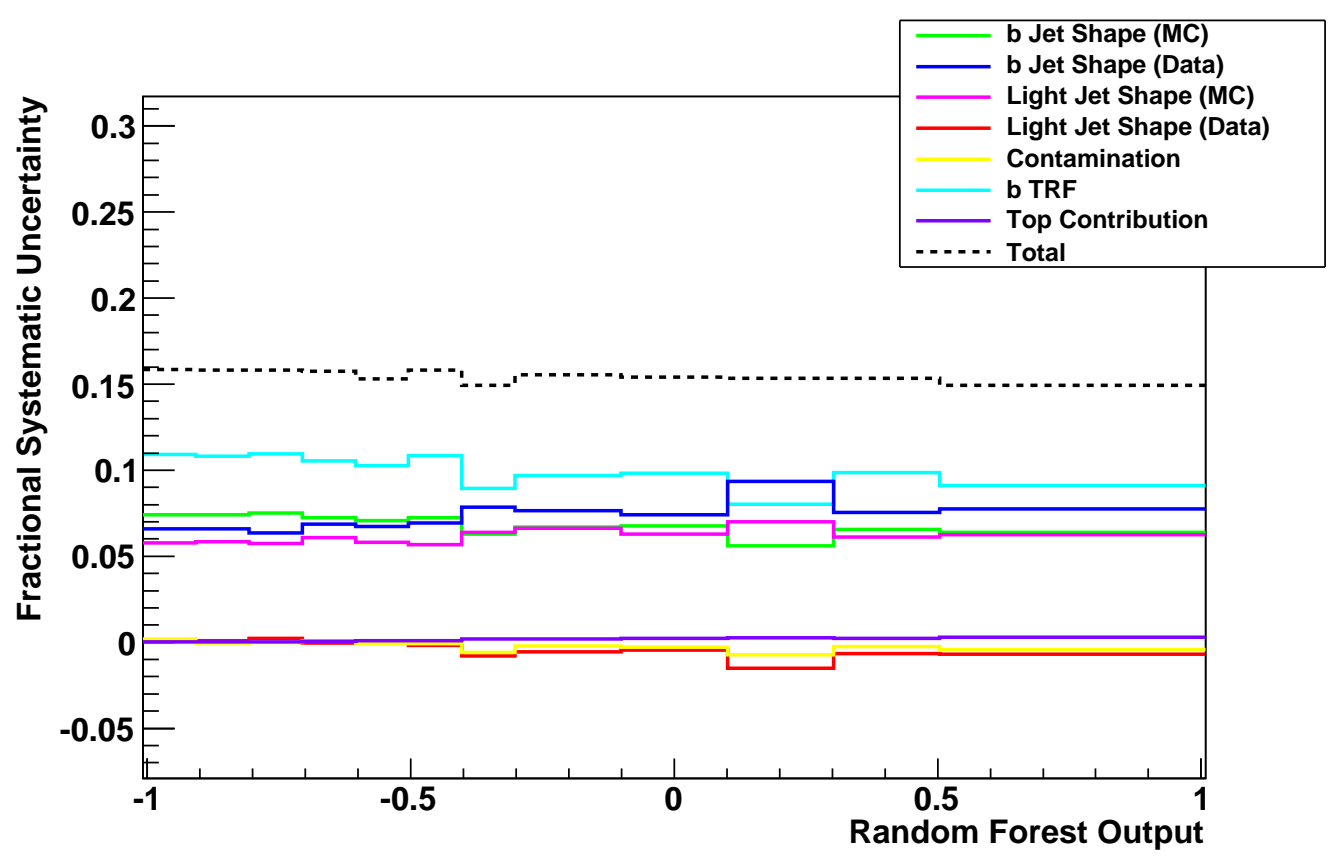

(a)

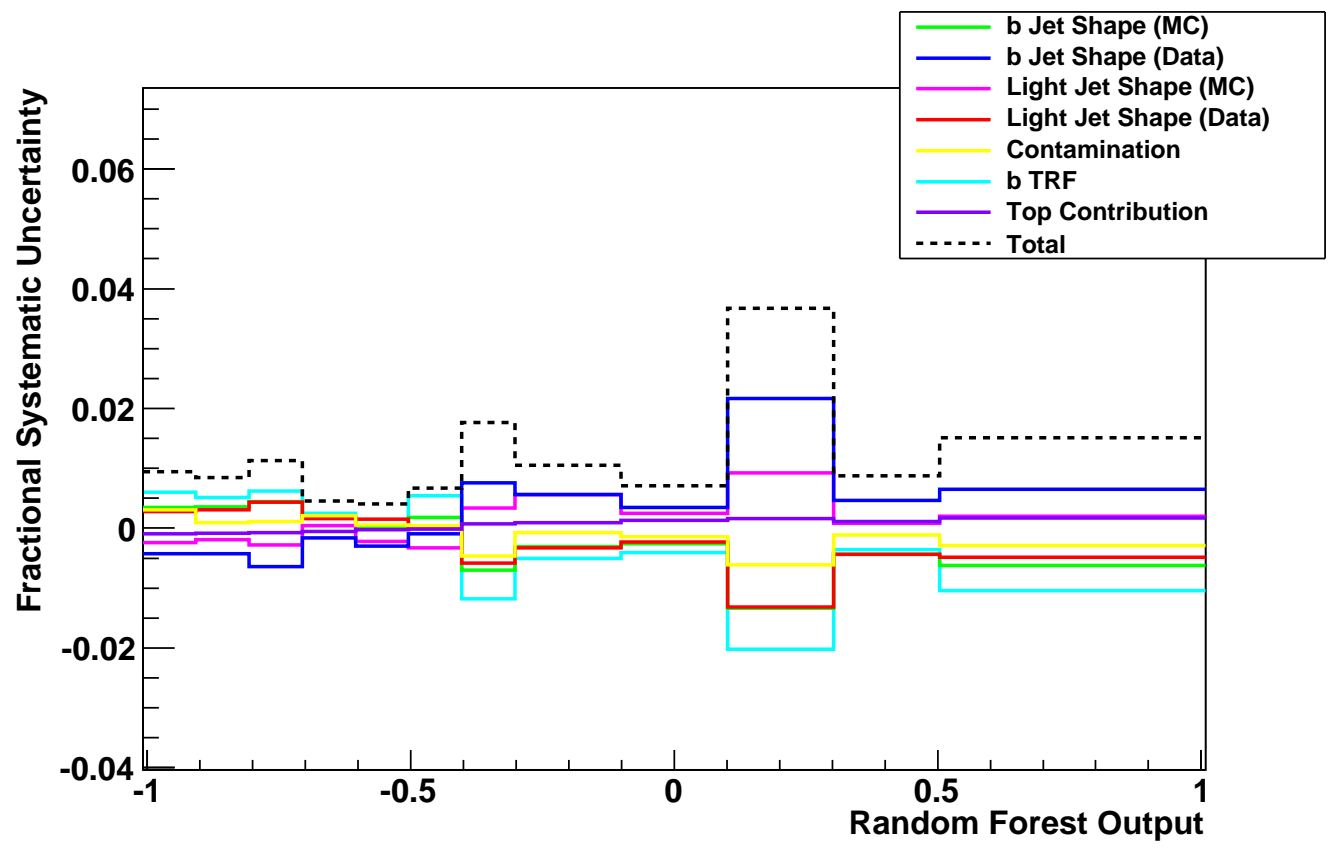

(b)

Figure 5.9: Systematic uncertainties as a fraction of the nominal predicted background plotted as a function of the p17 four jet channel RF output for a Higgs boson mass of $180 \mathrm{GeV}$. The systematics are shown both (a) un-normalised and (b) normalised to equal area with the predicted background. The black dotted line in (b) is intended to give an indication of the total uncertainty: this is not the true total because the scale of the nominal background is allowed to float in the limit setting process. 


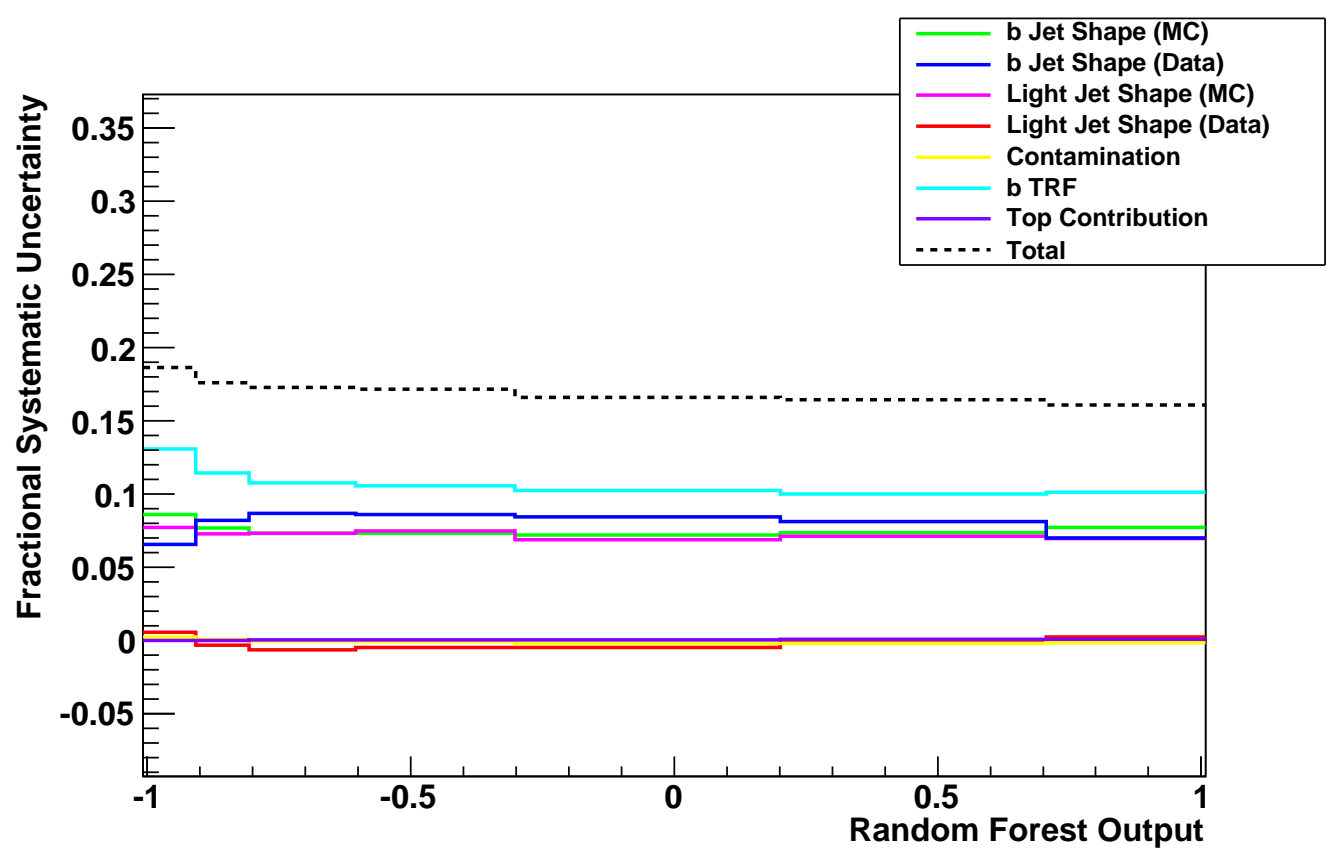

(a)

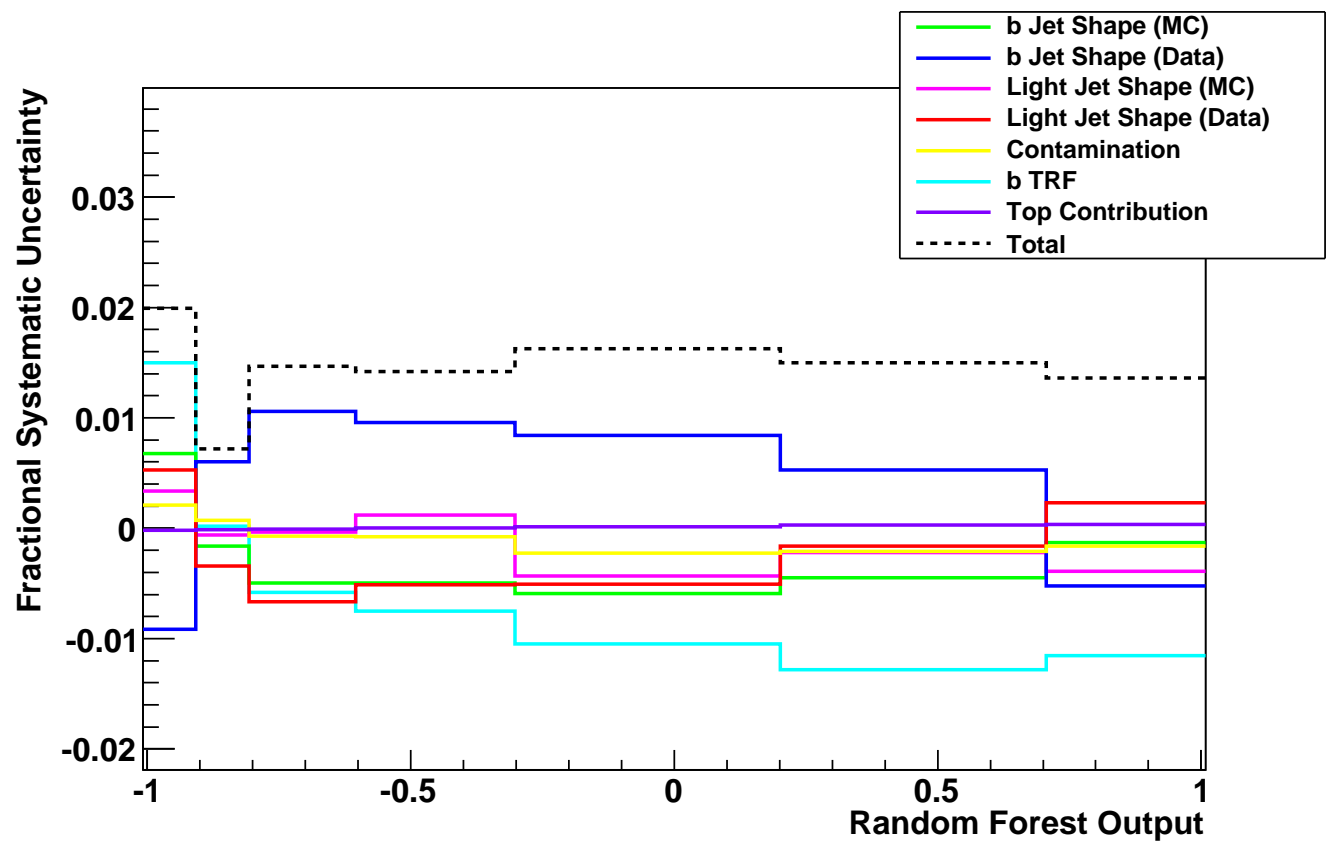

(b)

Figure 5.10: Systematic uncertainties as a fraction of the nominal predicted background plotted as a function of the p20 three jet channel RF output for a Higgs mass of $180 \mathrm{GeV}$. The systematics are shown both (a) un-normalised and (b) normalised to equal area with the predicted background. The black dotted line in (b) is intended to give an indication of the total uncertainty: this is not the true total because the scale of the nominal background is allowed to float in the limit setting process. 


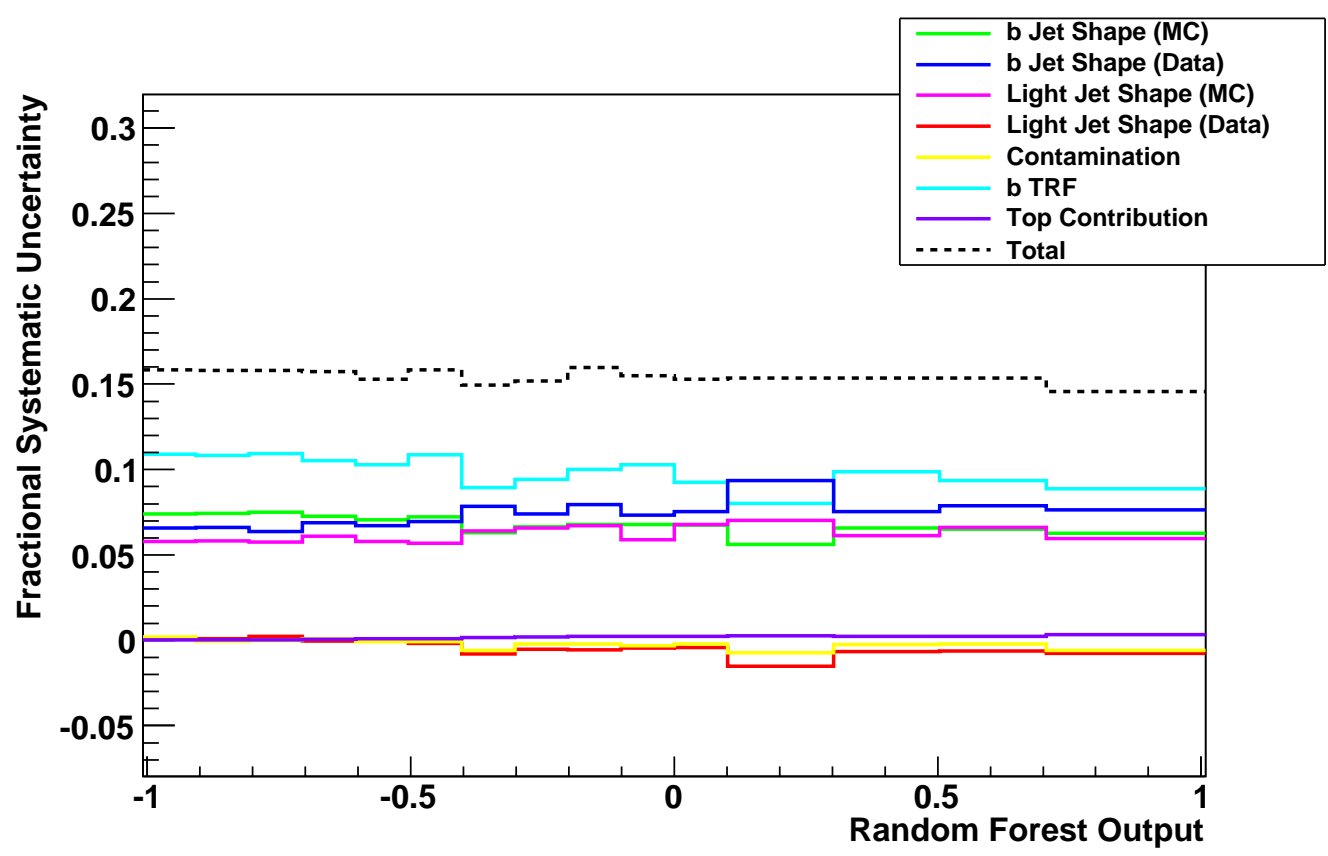

(a)

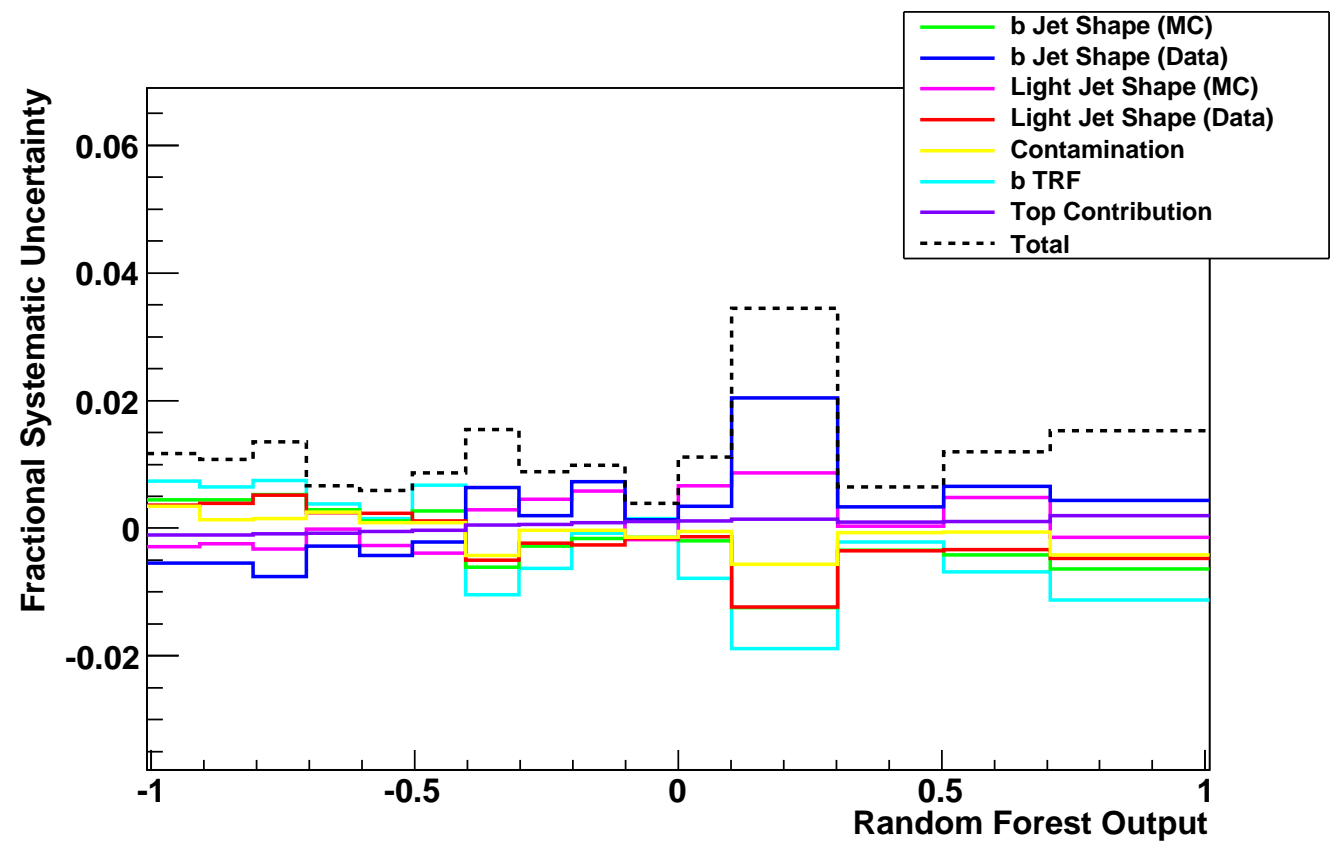

(b)

Figure 5.11: Systematic uncertainties as a fraction of the nominal predicted background plotted as a function of the p20 four jet channel RF output for a Higgs boson mass of $180 \mathrm{GeV}$. The systematics are shown both (a) un-normalised and (b) normalised to equal area with the predicted background. The black dotted line in (b) is intended to give an indication of the total uncertainty: this is not the true total because the scale of the nominal background is allowed to float in the limit setting process. 


\subsection{Results}

The results of the search were evaluated by setting confidence limits using the COLLIE package [85]. The RF output was used as the input observable; signal-plusbackground $\left(H_{\text {test }}\right)$ and background-only $\left(H_{\text {null }}\right)$ hypotheses are derived from this, and COLLIE calculates the log likelihood ratio (LLR) from these hypotheses:

$$
Q_{n}=-2 \ln \frac{P\left(n \mid H_{\text {test }}\right)}{P\left(n \mid H_{\text {null }}\right)}
$$

where $Q_{n}$ is the LLR for an outcome of $n$ events compared with the test and null hypotheses. This test statistic is summed over all bins of the distribution and each channel. The pure Poisson likelihood for an individual bin is given by:

$$
P(n \mid H)_{\text {Poisson }}=\frac{p^{n} e^{-p}}{n !}
$$

where $p$ is the number of events predicted by the hypothesis (to find the overall likelihood, the values of all individual likelihoods are multiplied together). All parameters that affect the hypothesis test apart from the parameter that is being investigated (in this case, the value of $\sigma \times \mathrm{BR}$ ) are termed nuisance parameters, so the systematic uncertainties are considered nuisance parameters. These are incorporated into the hypothesis test by multiplying the Poisson likelihood (5.2) by a Gaussian likelihood ratio. The LLR is then given by:

$$
Q_{n}=-2 \ln \left(\frac{(s+b)^{n} e^{-(s+b)}}{b^{n} e^{-b}} \times \prod_{i=1}^{N P} e^{-R_{i}^{2}}\right)
$$

where $s$ and $b$ are the predicted numbers of signal and background events respectively and $N P$ is the number of nuisance parameters for hypothesis $H . R_{i}$ is given by

$$
R_{i}=\frac{\theta_{i}-\theta_{i}^{0}}{\sigma_{i}}
$$

where $\theta_{i}, \theta_{i}^{0}$ and $\sigma_{i}$ are the varied value, central value and deviation of the nuisance parameter respectively. The effect of two example nuisance parameters, the heavy jet TRF uncertainty and the top quark pair contribution uncertainty, on the background and signal-plus-background LLRs is given in Figure 5.12. Systematics with little effect on the LLR (such as the $t \bar{t}$ uncertainty) produced flat distributions. Dominant sources of uncertainty, such as the heavy-jet TRF, produced distributions with a clear minima, indicating that these systematics constrained the value of the LLR. 


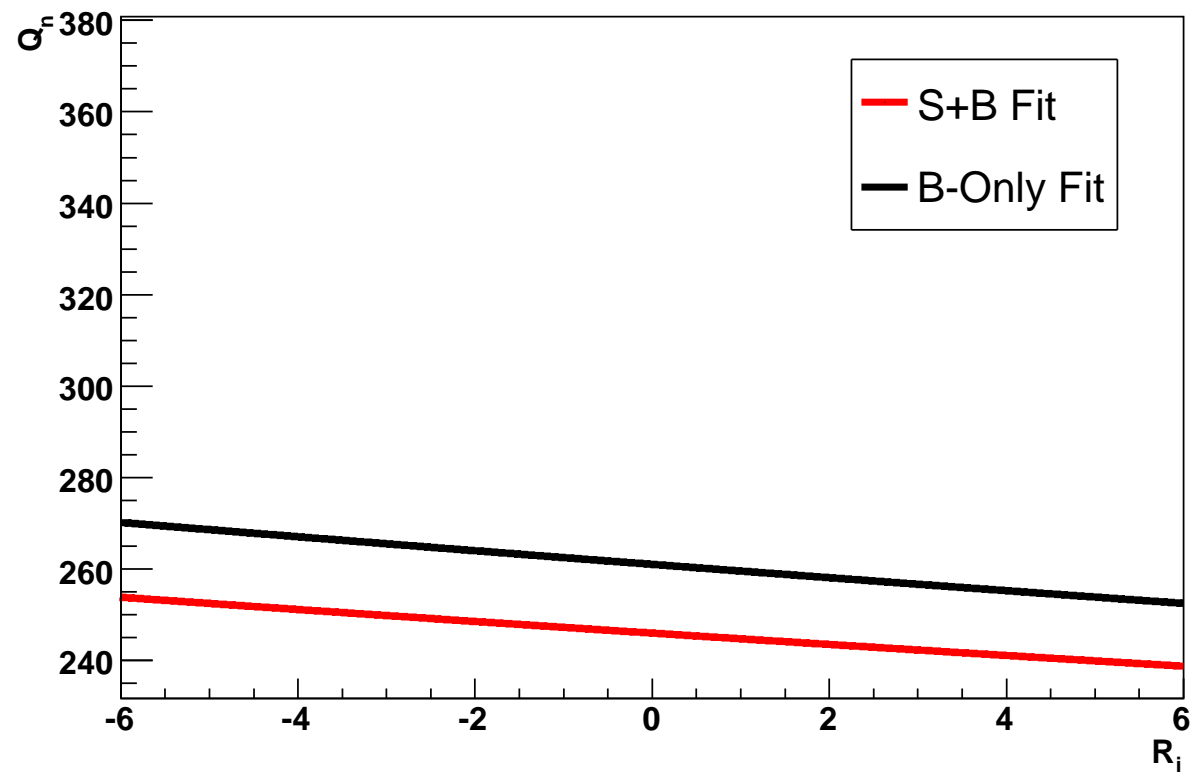

(a)

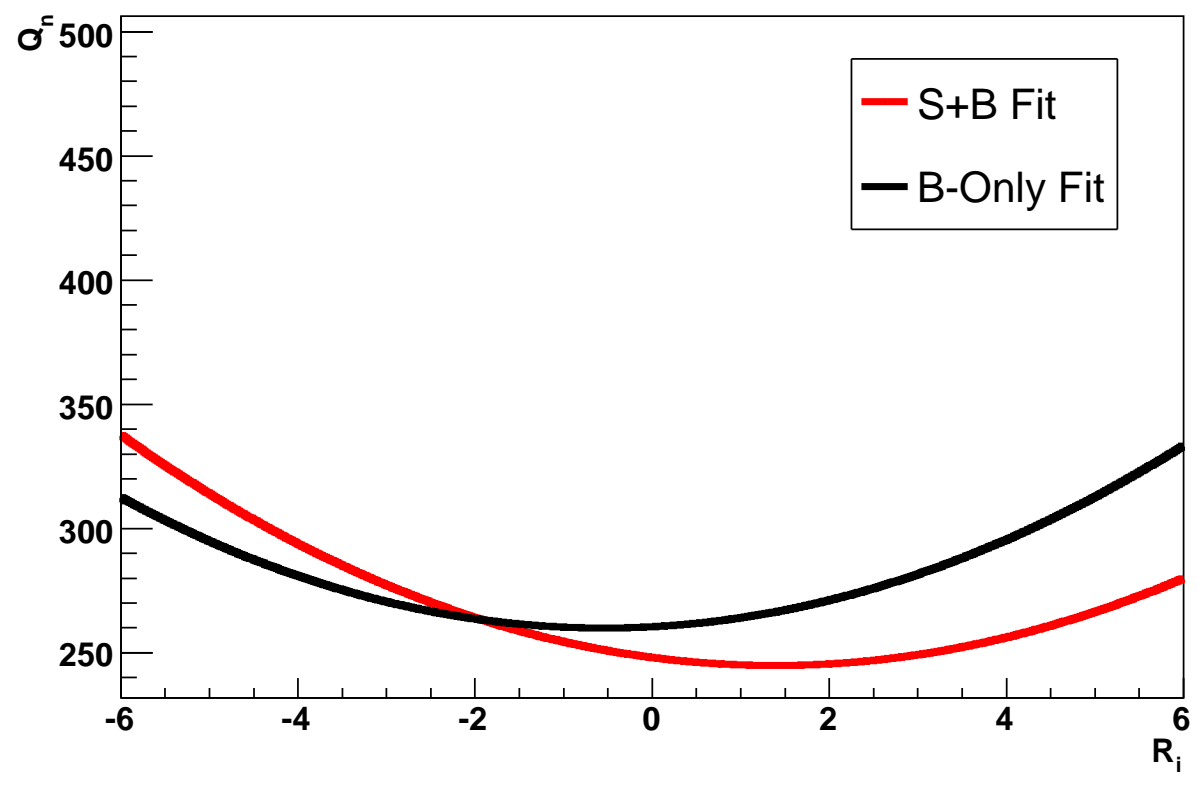

(b)

Figure 5.12: The impact of the value of $R_{i}(5.4)$ for (a) the $t \bar{t}$ background contribution and (b) the heavy jet TRF systematic uncertainties on the overall LLRs, $Q_{n}$ for signal-plus-background (red) and background-only (black) cases. In these plots, the values of all other nuisance parameters apart from the one under investigation are fixed. The signal hypothesis in both plots is for a Higgs boson with $m_{A}=160 \mathrm{GeV}$ and $\tan \beta=50$. The top quark contribution was a relatively small uncertainty, and varying this parameter did not have a large effect on $Q_{n}$. The heavy-jet TRF, on the other hand, was a dominant source of uncertainty, and the LLR was more sensitive to this parameter. The minima of the signal-plus-background and background only $Q_{n}$ distributions occur at different values of $R_{i}$ : this shows that this uncertainty has a different effect for the two hypotheses. 
The profile likelihood method [85] was used to minimise the effects of the nuisance parameters in this analysis.

The likelihood given in (5.3) was used to calculate a value of the test statistic $\left(Q_{d}\right)$ for data. Distributions of the test statistic for the background and signal-plusbackground cases were generated using Poisson distributions around the expected number of events, $b$ and $s+b$ respectively (in this analysis, the background normalisation was unknown, so the final discriminant distribution for the background was normalised to have equal area with the same distribution for data). The Poisson distributions were generated using a number of pseudo-experiments. The test statistic was summed over all bins of the final variable distribution, and over all channels. In the profile method, values of $\theta_{i}$ are found in each pseudo-experiment such that the fit to data is optimised. The values of $R_{i}$ for the two example nuisance parameters are plotted in Figure 5.13. The width of these distributions give some indication of how well the $\pm 1 \sigma$ fluctuations were estimated, while the mean shows the scale of the uncertainty that optimises the LLR.

Comparing the values of the LLR in Equation (5.1) for the background-only and signal-plus-background likelihoods gives an indication of the degree of separation between the signal and the background. Comparing the log likelihood value for data indicates which hypothesis is more similar to the data sample. The signalplus-background and background-only expected log likelihood ratios for different values of $m_{A}$ are plotted in Figure 5.14.

A frequentist interpretation of an $\mathrm{x} \%$ confidence limit is that as the number of pseudo-experiments tends to infinity, 1-x\% of pseudo-experiments will be more background-like than the data: the confidence limits for the signal-plus-background and background-only hypotheses are given, therefore, as:

$$
\begin{gathered}
C L_{s+b}=P\left(Q_{s+b} \geq Q_{d}\right) \\
C L_{b}=P\left(Q_{b} \geq Q_{d}\right)
\end{gathered}
$$

respectively. The results of this search are given in terms of $C L_{s}$, defined as:

$$
C L_{s}=\frac{C L_{s+b}}{C L_{b}}
$$

Defining $C L_{s}$ in this way (known as the 'modified frequentist method') reduces the risk of a false exclusion in samples where the background is poorly understood: in these samples, both the test and null hypotheses could be excluded, leading to a strong exclusion in terms of $C L_{s+b}$ but not in $C L_{s}$. The test hypothesis was adjusted 


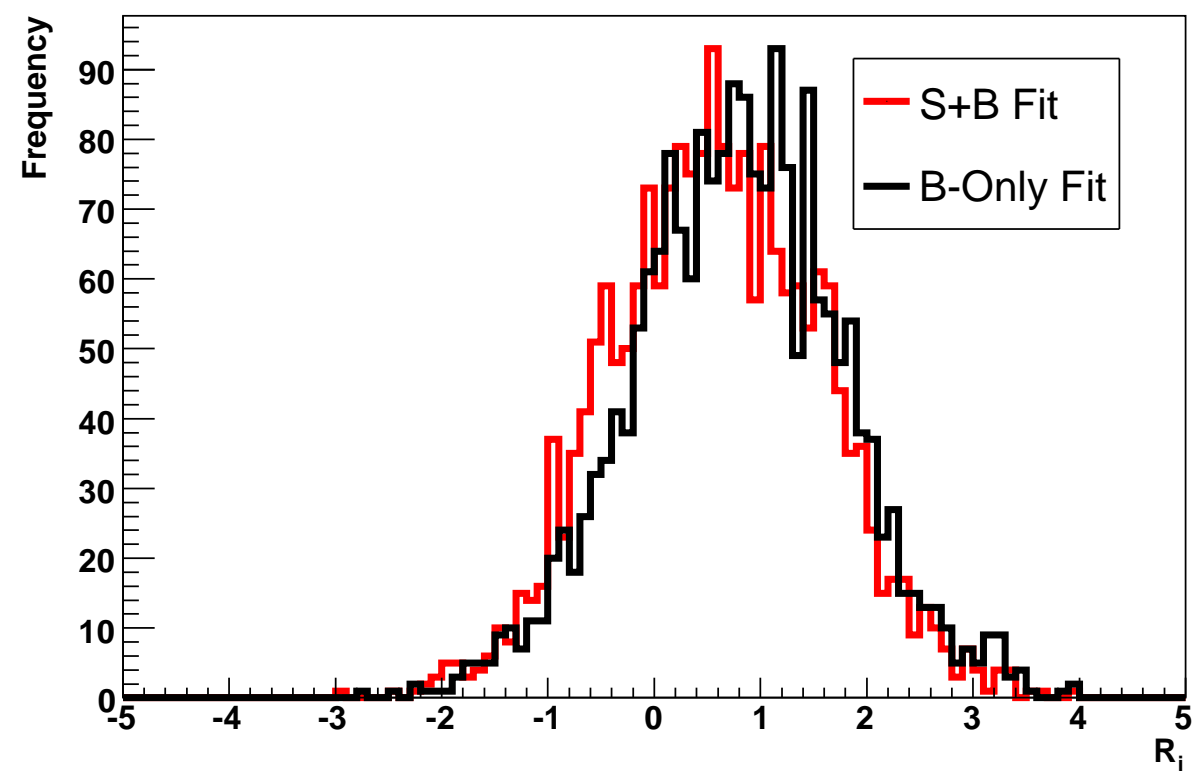

(a)

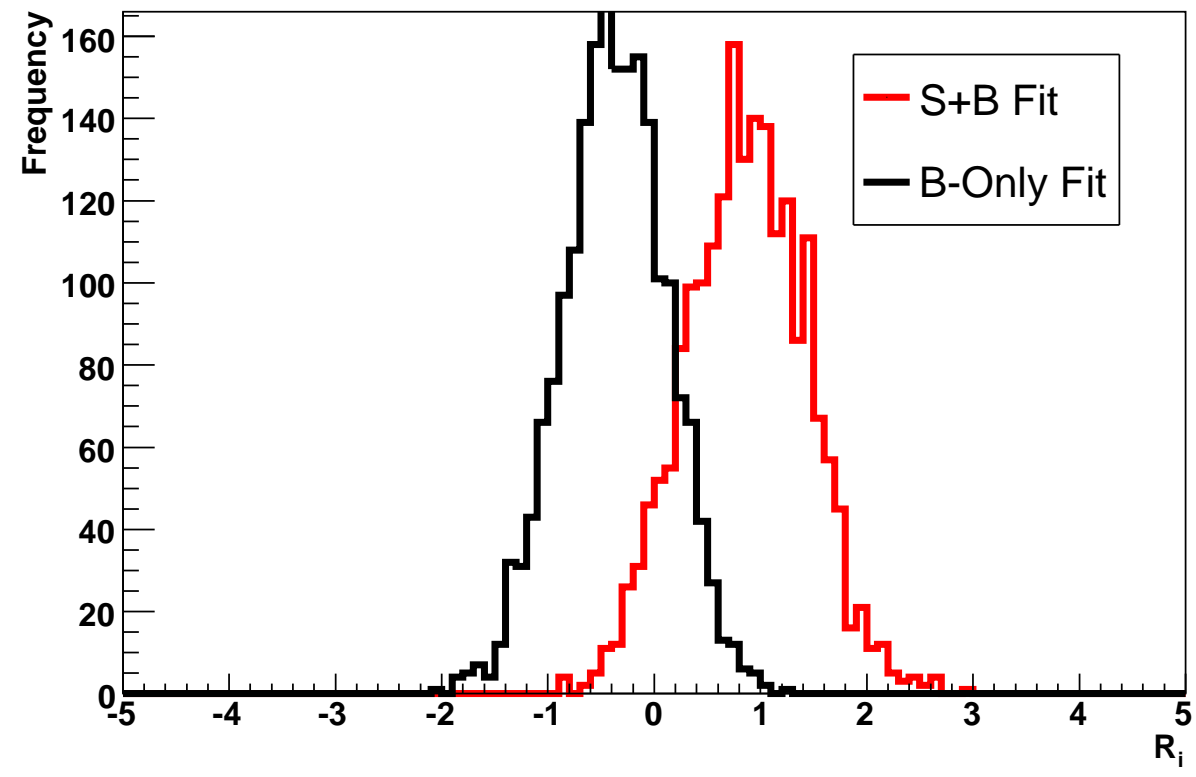

(b)

Figure 5.13: The values of $R_{i}(5.4)$ for (a) the top quark contribution and (b) the the heavy jet TRF uncertainties in each pseudo-experiment: these values optimise the overall likelihood. Note that, unlike in Figure 5.12, all parameters were allowed to fluctuate when minimising the likelihood. The width of the $R_{i}$ distributions in (a) is roughly 1 . The mean of the distributions in (a) is between 0.5 and 0.8 ; this indicates that the likelihood was optimised for a central value slightly higher than the nominal estimate. In the heavy jet TRF uncertainty plots, the widths of the distributions are less than one: this indicates that the fit constrains the value of this uncertainty. The signal and signal-plus-background distributions peak at different values of $R_{i}$, as they did in Figure 5.12 (b). 


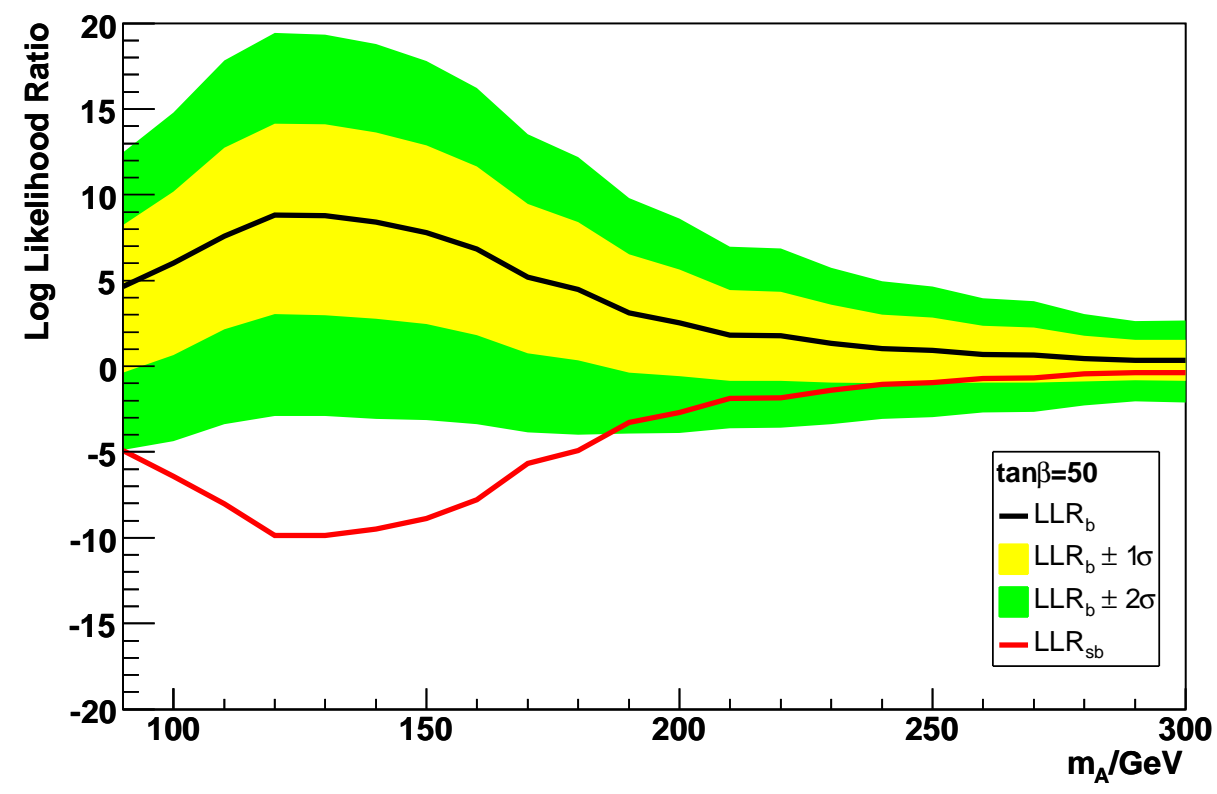

Figure 5.14: The LLR for the expected number of background-only (black) and signal-plusbackground (red) events plotted against $m_{A}$. The deviation of the two distributions indicates the degree of separation between the signal and background hypotheses. The yellow and green bands show the value of the LLR for the background-only hypothesis where the predicted number of background events has been increased and decreased by one and two standard deviations respectively: these allow the mean value of the signal-plus-background LLR distribution to be compared with the width of the background-only LLR distribution. The signal has been scaled according to Equation (3.1) with $\tan \beta=50$. 
by varying the signal $\sigma \times B R$ until $C L_{s}$ was equal to 0.05 , thus excluding this value of $\sigma \times B R$ with $95 \%$ confidence.

As discussed in Chapter 3, the background normalisation was not known in this analysis. This was accounted for by adding an unconstrained nuisance parameter for each channel which was fitted to the data. The normalisation scale factor (the central value of the nuisance parameter) was set to such a value that the number of background events was equal to the number of data events. The normalisation was allowed to vary: the size of the $\pm 1 \sigma$ fluctuations was estimated using the $\Delta \chi^{2}=$ $\chi^{2}-\chi_{\min }^{2}$ distribution.

The expected confidence limits are discussed in sections 5.3.1 and 5.3.2.

\subsubsection{Model Independent and Naive MSSM Confidence Lim- its}

The expected confidence limits on $\sigma \times B R$ are plotted versus $m_{A}$ in Figure 5.15, where $m_{A}$ is the mass of two of the neutral Higgs bosons. Using the enhancement in Equation (3.1), and disregarding any increase in Higgs boson width or effects from SUSY corrections, the $\sigma \times B R$ limits can be used to place naive limits on the $\left(m_{A}\right.$, $\tan \beta)$ parameter space. The excluded values of $\sigma \times B R$ limits are given in Table 5.1, and plotted in Figure 5.15(a) as a function of $m_{A}$. The exclusion of $\sigma \times B R$ for the $\phi b \rightarrow b \bar{b} b$ process was taken as a measure of sensitivity. The exclusion compares favourably with that from the previous $\left(5.2 \mathrm{fb}^{-1}\right)$ version of the analysis, as shown in Figure 5.16. This improvement is due to three aspects: the increased amount of data, the increased efficiency of the MVA b-tagger in comparison to the NN btagger, and the change in method (described in Chapter 4). By changing these one at a time, it was found that adding $1.4 \mathrm{fb}^{-1}$ of data improved the sensitivity by roughly $12 \%$. The improvement due to the MVA b-tagger was approximately $17 \%$. The improvement due to the change in analysis method varied as a function of $m_{A}$; the improvement was about $30 \%$ at low mass, but was less pronounced at higher masses where the invariant mass was the most powerful discriminant.

The effect of the systematic uncertainties on the sensitivity of the analysis were determined by doubling the background systematics. The resulting exclusion limits are shown in Figure 5.17: the expected sensitivity decreases by roughly $20 \%$ when the systematics are doubled. This decrease in sensitivity is relatively small considering the large increase in the systematic uncertainties.

The MSSM can parametrised by two values at leading SUSY order: at higher orders, the properties of the Higgs sector are affected by over 100 parameters. The method used to set limits on the MSSM Higgs sector with SUSY loop corrections is described in section 5.3.2. 


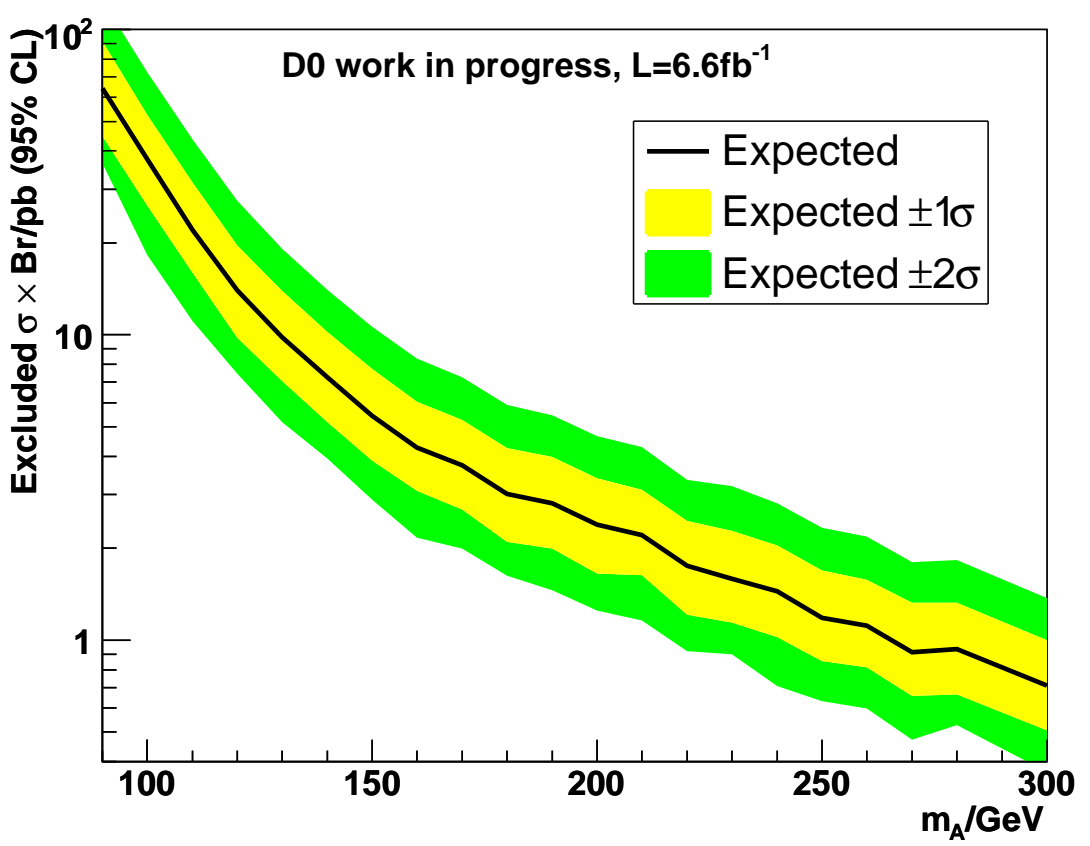

(a)

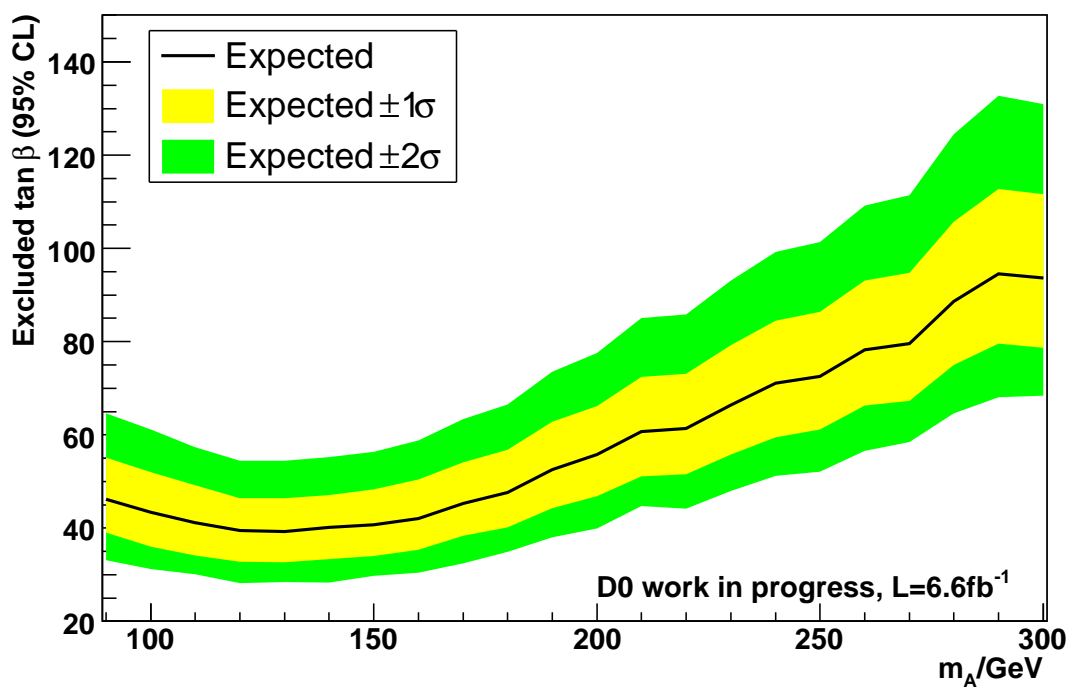

(b)

Figure 5.15: Expected confidence limits plotted versus $m_{A}$ : (a) the values of $\sigma \times B R$ for the $\phi b \rightarrow b \bar{b} b$ process and (b) $\tan \beta$ excluded to $95 \%$ confidence, as a function of neutral Higgs boson mass $\left(m_{A}\right)$. The $\tan \beta$ limit is calculated using Equation (3.1). The yellow and green bands represent the $\pm 1 \sigma$ and $\pm 2 \sigma$ variations around the expectation. 


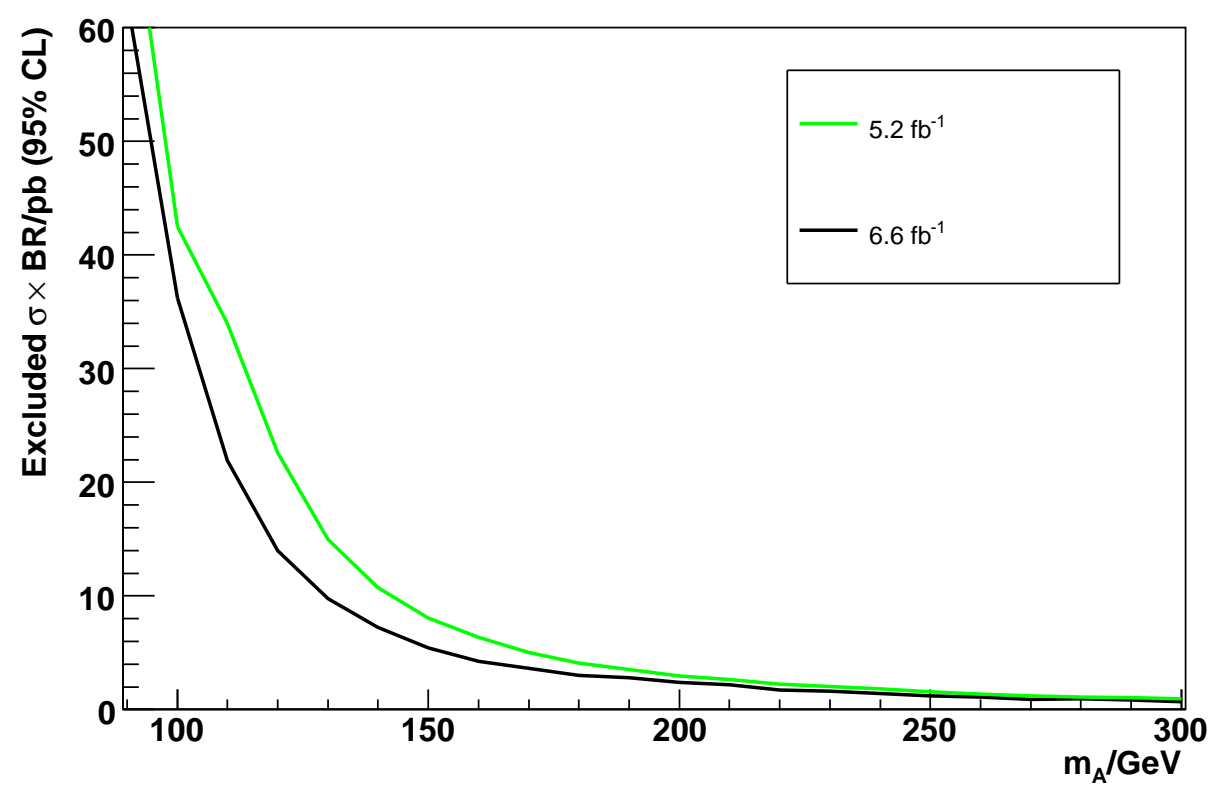

(a)

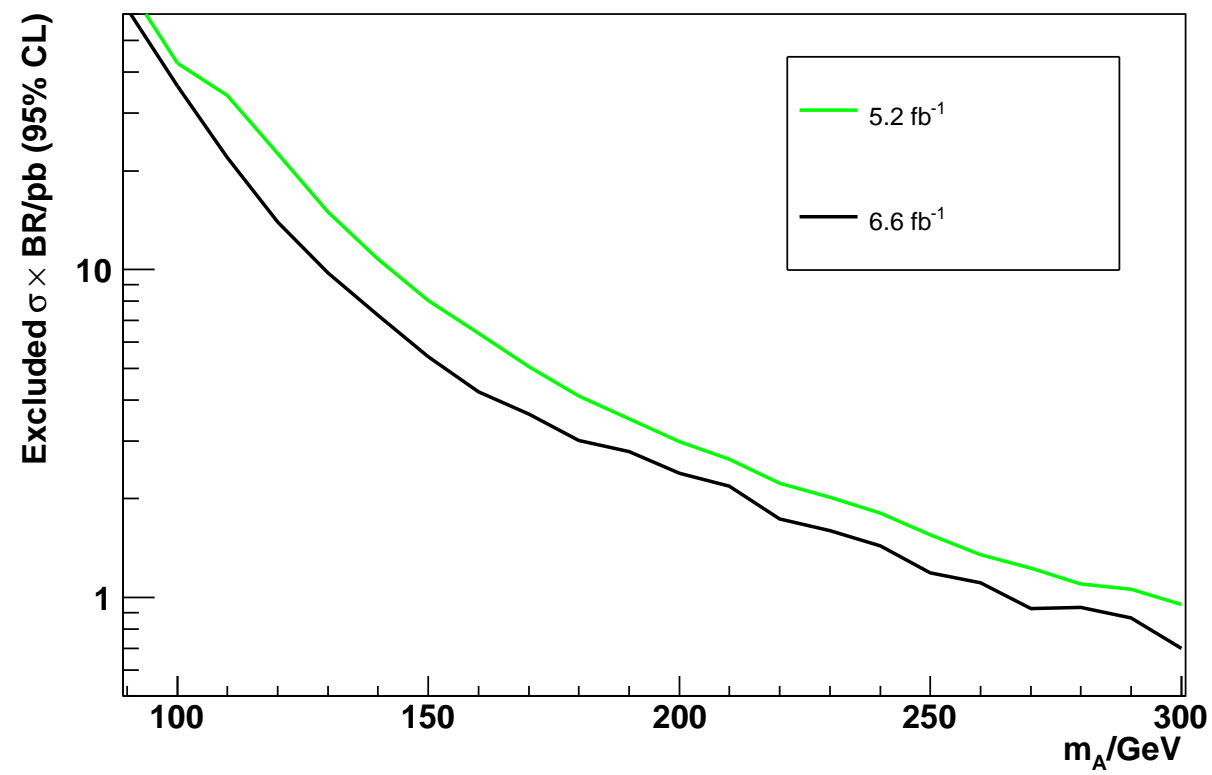

(b)

Figure 5.16: Comparison of expected confidence limits on $\sigma \times$ BR for $5.2 \mathrm{fb}^{-1}$ (green) and 6.6 $\mathrm{fb}^{-1}$ (black), plotted on (a) a linear and (b) a logarithmic scale. The increase in sensitivity is greater than that which would be expected for the increase in integrated luminosity analysed. 


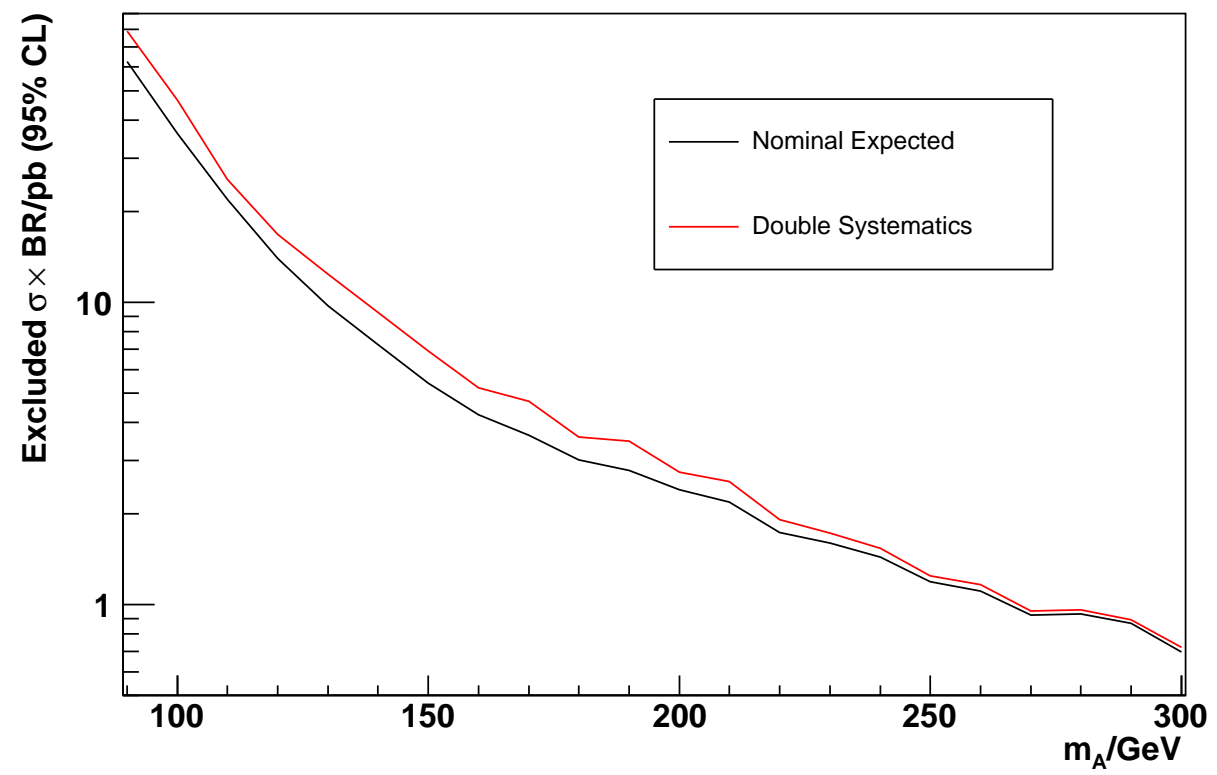

Figure 5.17: Comparison of expected confidence limits on $\sigma \times \mathrm{BR}$ for $6.6 \mathrm{fb}^{-1}$ (black) and the same with doubled systematics. The decrease in sensitivity is roughly $20 \%$. 


\begin{tabular}{|c|c|c|c|c|c|c|}
\hline \multirow[b]{2}{*}{$m_{A} / \mathrm{GeV}$} & \multirow[t]{2}{*}{ Observed [pb] } & \multicolumn{5}{|c|}{ Expected [pb] } \\
\hline & & $-2 \sigma$ & $-1 \sigma$ & median & $+1 \sigma$ & $+2 \sigma$ \\
\hline 90 & - & 31.53 & 44.43 & 62.42 & 89.06 & 122.20 \\
\hline 100 & - & 18.71 & 25.35 & 36.15 & 52.03 & 72.01 \\
\hline 110 & - & 11.64 & 15.09 & 21.83 & 31.27 & 42.34 \\
\hline 120 & - & 7.04 & 9.63 & 13.93 & 19.44 & 26.80 \\
\hline 130 & - & 5.10 & 6.81 & 9.77 & 13.62 & 18.72 \\
\hline 140 & - & 3.77 & 5.02 & 7.23 & 10.02 & 13.67 \\
\hline 150 & - & 2.79 & 3.76 & 5.42 & 7.60 & 10.40 \\
\hline 160 & - & 2.23 & 2.96 & 4.24 & 6.08 & 8.33 \\
\hline 170 & - & 1.88 & 2.61 & 3.64 & 5.18 & 7.13 \\
\hline 180 & - & 1.56 & 2.15 & 2.98 & 4.22 & 5.82 \\
\hline 190 & - & 1.42 & 1.95 & 2.76 & 3.93 & 5.41 \\
\hline 200 & - & 1.22 & 1.67 & 2.35 & 3.34 & 4.59 \\
\hline 210 & - & 1.13 & 1.55 & 2.19 & 3.09 & 4.25 \\
\hline 220 & - & 0.90 & 1.23 & 1.73 & 2.45 & 3.35 \\
\hline 230 & - & 0.82 & 1.13 & 1.58 & 2.28 & 3.12 \\
\hline 240 & - & 0.75 & 1.01 & 1.42 & 2.02 & 2.79 \\
\hline 250 & - & 0.63 & 0.85 & 1.19 & 1.68 & 2.32 \\
\hline 260 & - & 0.58 & 0.78 & 1.11 & 1.57 & 2.16 \\
\hline 270 & - & 0.48 & 0.65 & 0.92 & 1.31 & 1.81 \\
\hline 280 & - & 0.49 & 0.66 & 0.93 & 1.32 & 1.83 \\
\hline 290 & - & 0.46 & 0.62 & 0.86 & 1.23 & 1.69 \\
\hline 300 & - & 0.37 & 0.50 & 0.70 & 0.99 & 1.36 \\
\hline
\end{tabular}

Table 5.1: Values of $\sigma \times$ branching ratio excluded with $95 \%$ confidence for $6.6 \mathrm{fb}^{-1}$ of D $\varnothing$ Run II data for different values of $m_{A}$. The excluded values of $\sigma \times B R$ for $\pm 1 \sigma$ and $\pm 2 \sigma$ around the expectation are also given. 


\subsubsection{Confidence Limits for Benchmark MSSM Scenarios}

Rather than setting limits on a multi-dimensional parameter space, a common strategy followed by experimental searches is to use agreed scenarios [87] in which the majority of relevant parameters are fixed, but $\tan \beta$ and $m_{A}$ may vary. This allows limits to be set on the $\left(m_{A}, \tan \beta\right)$ parameter space in the context of the scenario.

The two scenarios investigated in this analysis are the Maximum $m_{h}$ (' $m_{h}^{\max }$ ') and no-mixing scenarios. In the $m_{h}^{\max }$ scenario, the parameters are set such that the lightest supersymmetric Higgs, the h, has the highest possible mass. In the no-mixing scenario, the parameters are largely the same as the $m_{h}^{\max }$ scenario, but mixing is suppressed in the stop sector. The parameters in these scenarios are:

- $m_{h}^{\max }: M_{S U S Y}=1 \mathrm{TeV}, M_{2}=200 \mathrm{GeV}, X_{t}>0, A_{b}=A_{t}, m_{\tilde{g}}=0.8 M_{S U S Y}$

- No Mixing: $M_{S U S Y}=2 \mathrm{TeV}, M_{2}=200 \mathrm{GeV}, X_{t}=0, A_{b}=A_{t}, m_{\tilde{g}}=0.8 M_{S U S Y}$

where $M_{S U S Y}$ is the squark mass scale, $M_{2}$ is the gaugino mass term, $X_{t}$ is the stop mixing parameter, $A_{t}$ and $A_{b}$ are the trilinear coupling of the stop and sbottom sectors respectively, and $m_{\tilde{g}}$ is the gluino mass.

These scenarios are sensitive to the sign of the Higgs mixing parameter, $\mu$. To take this into account, the above scenarios were tested with $\mu$ set to \pm 200 . At higher orders, the Higgs coupling to b-quarks is sensitive to squark loop properties: this can be taken into account by using a modified ('effective') $\tan \beta$, given by:

$$
\tan \beta \rightarrow \tan \beta_{\text {eff }}=\frac{\tan \beta}{1+\Delta_{b}}
$$

where $\Delta_{b}$ is the correction due to stop-higgsino and sbottom-gluino loops. $\Delta_{b}$ varies according to the MSSM scenario, so the effective $\tan \beta$ is different in each scenario. Figure 5.18 shows the effective $\tan \beta$ plotted against $\tan \beta$ for each scenario: note that the correction varies with $\tan \beta$. The effect on the $\sigma \times B R$ enhancement is shown in Figure 5.19: the coloured lines show the signal random forest output distributions for the $m_{h}^{\max }$ and no mixing scenarios for positive and negative $\mu$, divided by the same distribution for signal with the naive enhancement. The value of $\tan \beta_{\text {eff }}$ was evaluated using calculations from the program FeynHiggs version 2.6.3 [86]. For the remainder of this Section, $\tan \beta_{\text {eff }}$ will be referred to as $\tan \beta$.

Another effect that must be considered is that the Higgs boson width increases due to the $\tan ^{2} \beta$ coupling enhancement: in the model independent and naive enhancement cases, the width was assumed to be small in comparison with the detector resolution. The effect on parameter $x$ at test mass $m_{\text {test }}$ can be modelled by 
convoluting the $x$ distributions for all mass hypotheses with a Breit Wigner mass distribution centred at $m_{\text {test }}$. This principle is demonstrated for the signal di-jet invariant mass distribution in the $m_{h}^{\max }(\mu=-200 \mathrm{GeV})$ scenario in Figure 5.20. The Figure shows the contribution of various mass hypotheses to the increased width mass peak. This method was applied to the signal RF output distributions to predict the RF response to Higgs boson events where the Higgs width is greater than detector resolution: the resulting $\mathrm{RF}$ distributions for each scenario, along with the naive enhancement of Equation (3.1), in Figure 5.19.

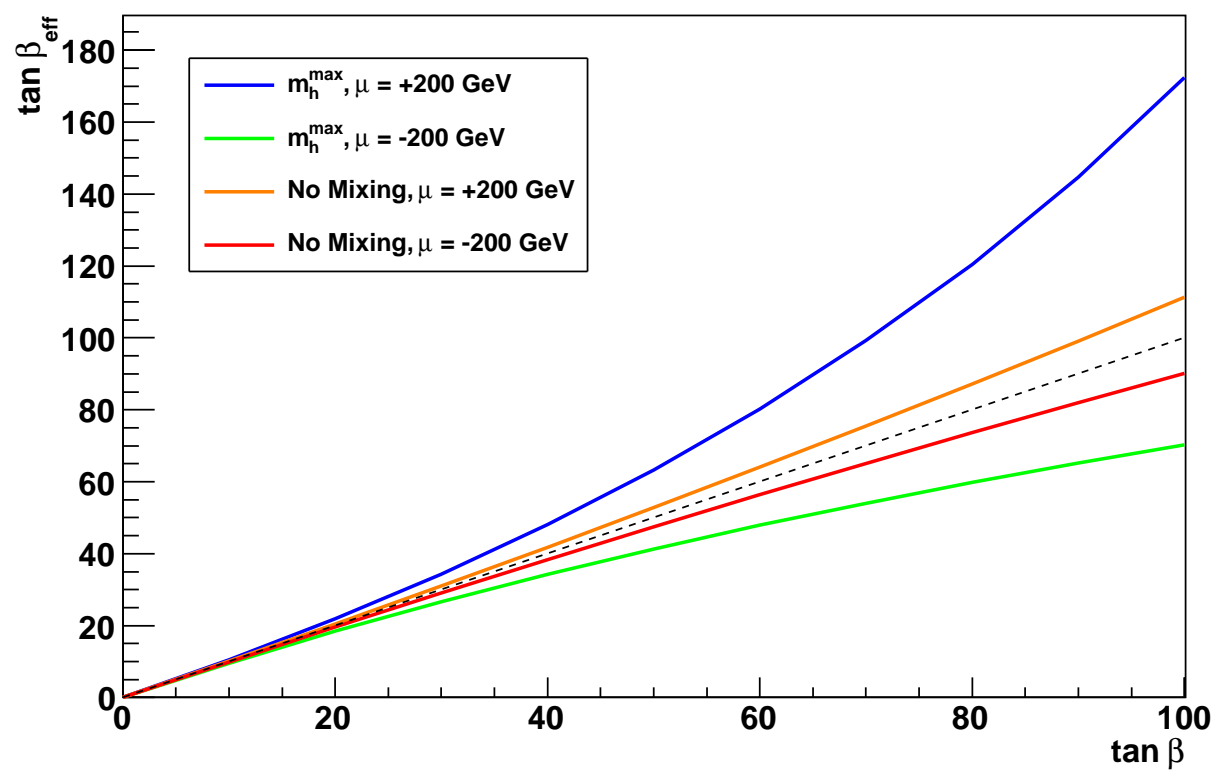

Figure 5.18: The effective $\tan \beta$ plotted against nominal $\tan \beta$ for the MSSM scenarios considered in this thesis. The correction to $\tan \beta$ is given in (5.8). The black dotted line shows the uncorrected value of $\tan \beta$ (that is, the case where $\Delta_{b}=0$ for all $\tan \beta$ ).

The expected confidence limits that were set using these MSSM scenario signal distributions are included in Figures 5.21 to 5.23. The Higgs boson width increases with $\tan \beta$. In the $m_{h}^{\max }, \mu=-200$ scenario with a Higgs boson mass of $180 \mathrm{GeV}$, the width is $4.6 \mathrm{GeV}$ for a $\tan \beta$ of 30 : this rises to a width of $110.4 \mathrm{GeV}$ for a $\tan \beta$ of 100 . At some value of $\tan \beta$, therefore, the signal resonance becomes too wide to distinguish from the background. For this reason, the limits, are less reliable at high $\tan \beta$. Additionally the MSSM becomes non-perturbative for values of $\tan \beta$ around 100 (the exact value is scenario-dependent), so limits on $\tan \beta$ above this value can be ignored. The expected $\tan \beta$ limit for the $m_{h}^{\max }(\mu=+200 \mathrm{GeV})$ scenario was above 100 for a large range of $m_{A}$ due to the large signal width and the high value of effective $\tan \beta$, so this limit is not shown. 


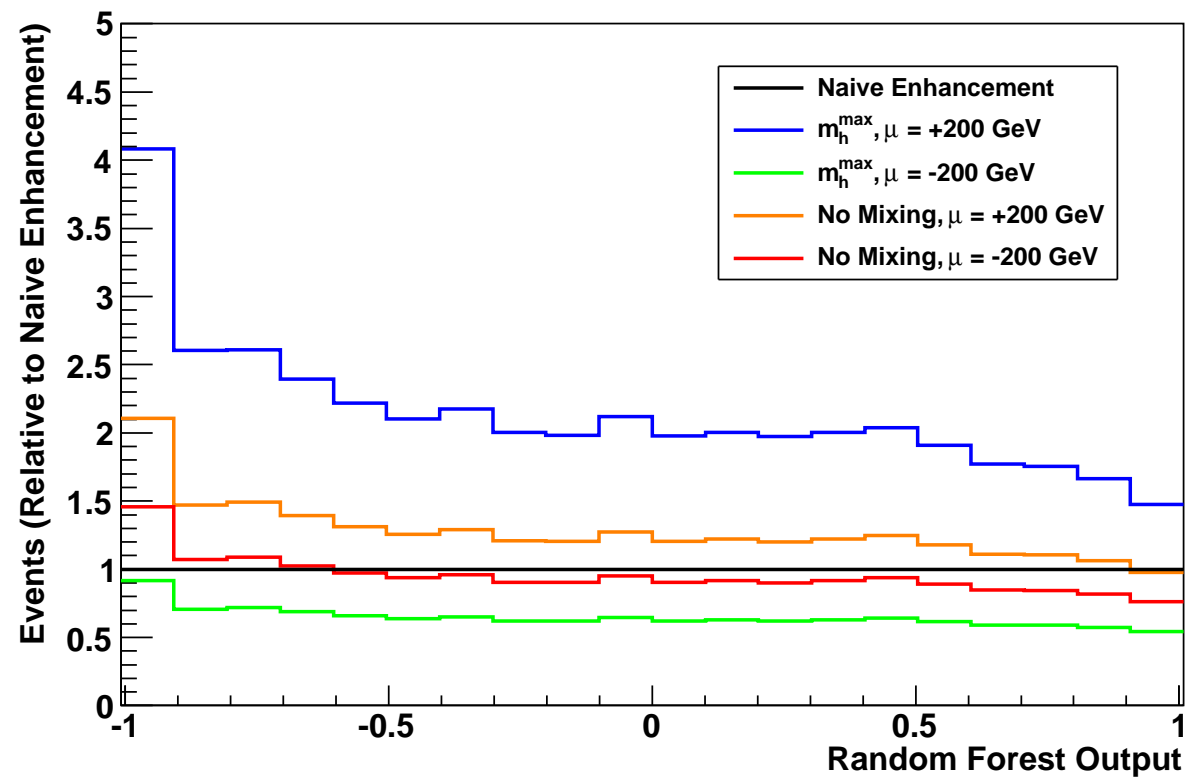

Figure 5.19: Signal RF distributions for $m_{A}=180 \mathrm{GeV}, \tan \beta=60$, for different MSSM scenarios relative to the naive enhancement. The corrections to $\tan \beta$ affect the scale of the distribution. The increased Higgs boson width causes more signal events to be placed in the most background-like bins of the RF distribution.

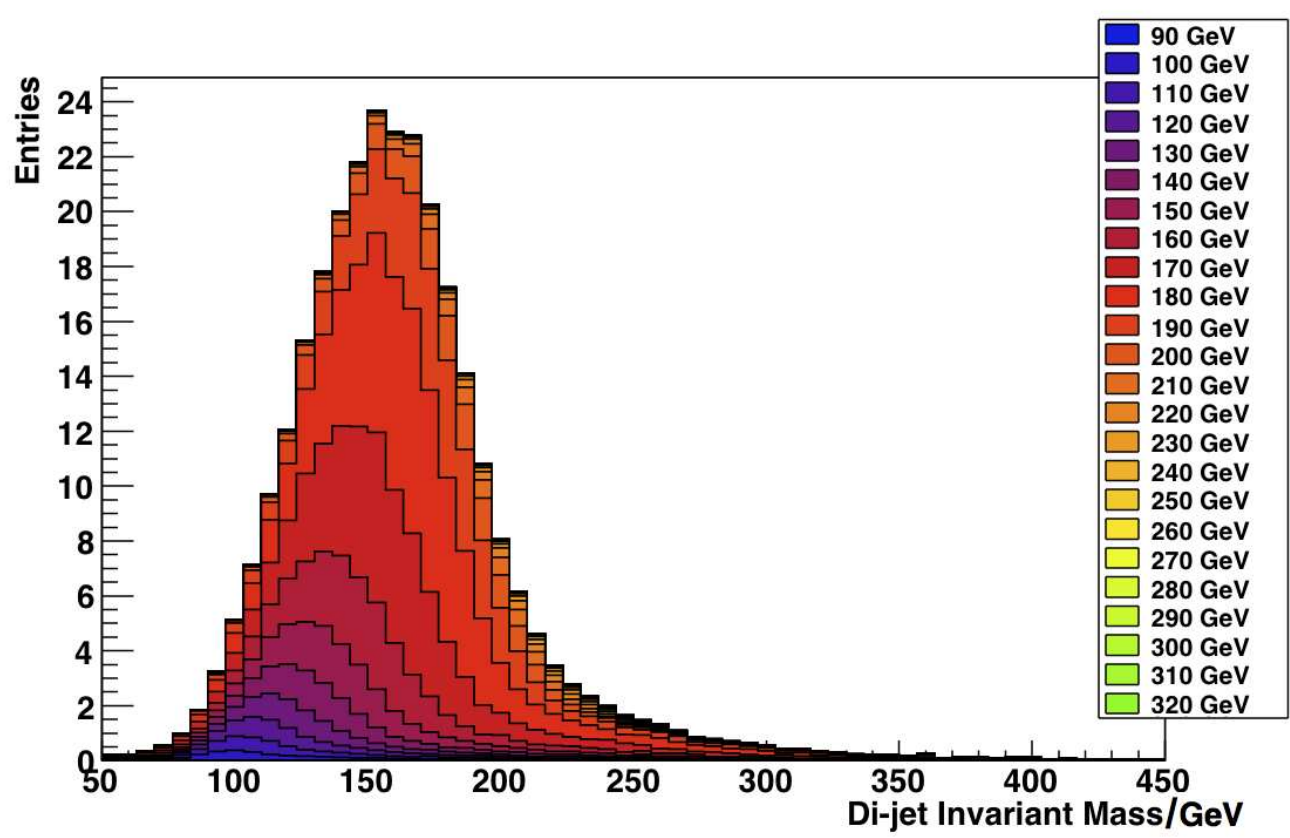

Figure 5.20: Signal di-jet invariant mass distribution for the leading jet pair, for a Higgs boson mass of $180 \mathrm{GeV}$, with a $\tan \beta$ of 60 in the $m_{h}^{\max }, \mu=-200 \mathrm{GeV}$ scenario. The predicted Higgs boson width for this scenario is approximately $25 \mathrm{GeV}$ : the width observed in is greater due to detector resolution effects. The contributions from the different mass hypotheses to the overall distribution are shown separately. The mass hypotheses closer to the test mass $(180 \mathrm{GeV})$ give the largest contribution, as expected. 


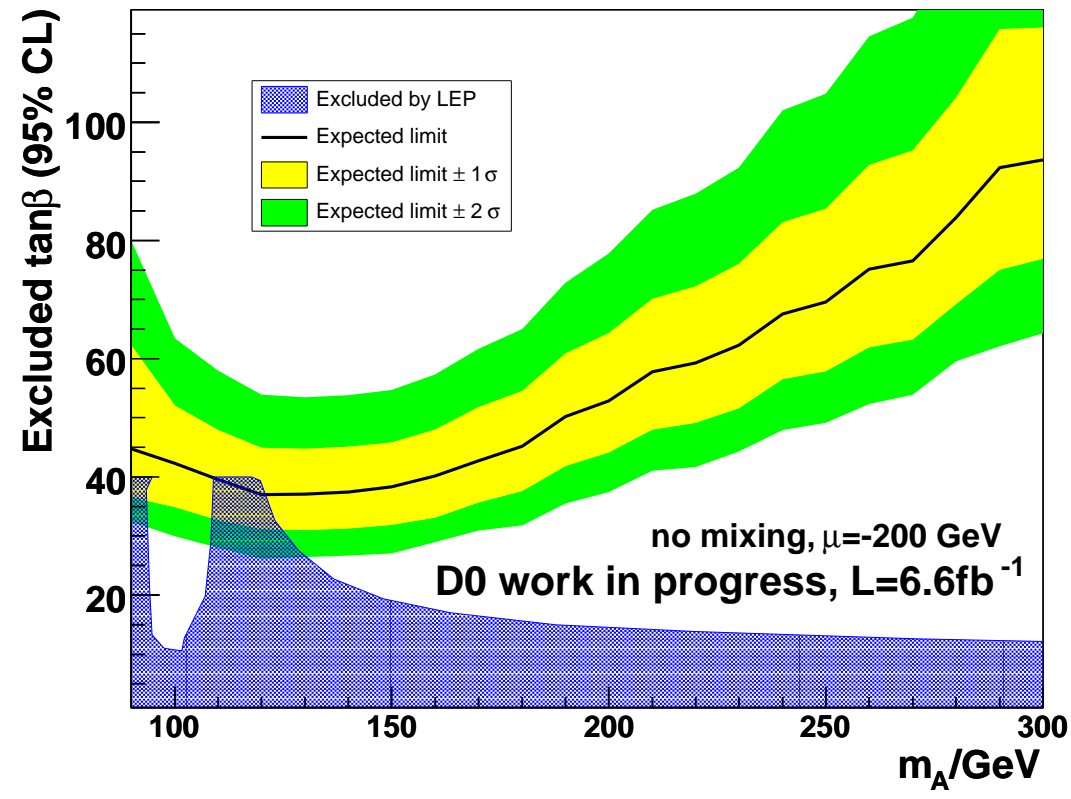

Figure 5.21: Expected confidence limits on $\tan \beta$ as a function of $m_{A}$, assuming the MSSM no mixing scenario $(\mu=-200 \mathrm{GeV})$. The yellow and green bands represent the $\pm 1 \sigma$ and $\pm 2 \sigma$ variations around the expectation. The region excluded by the LEP experiments is also shown [33].

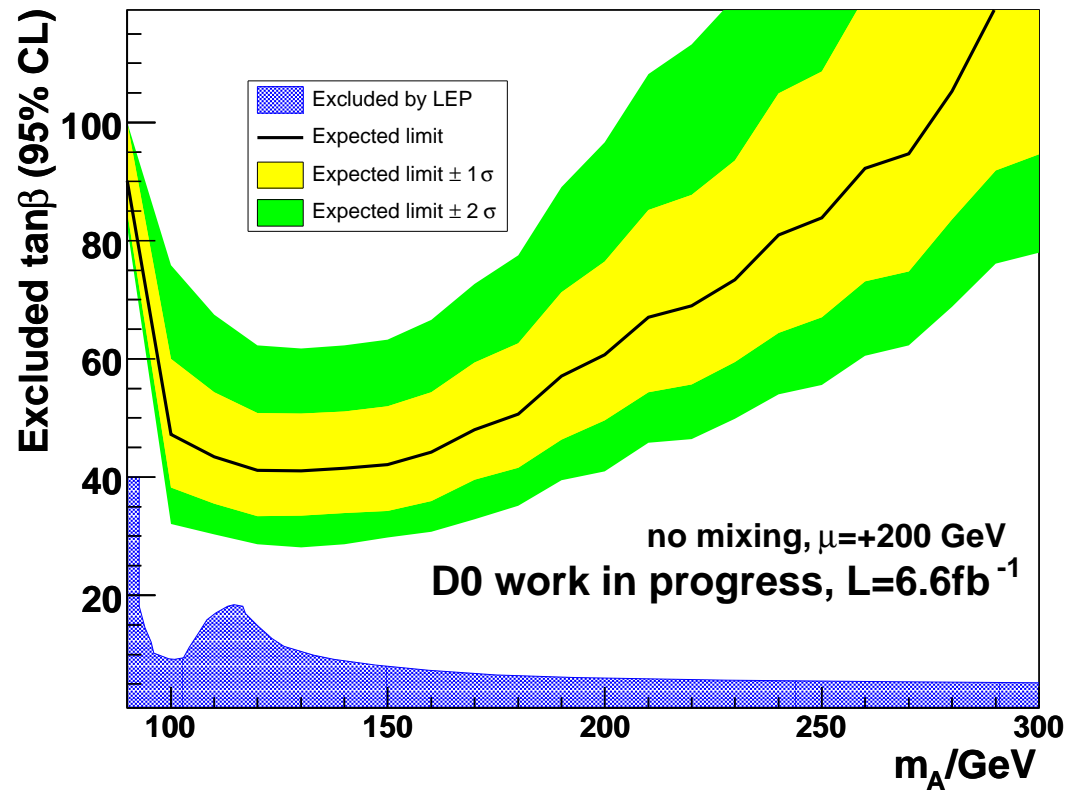

Figure 5.22: Expected confidence limits on $\tan \beta$ as a function of $m_{A}$, assuming the MSSM no mixing scenario $(\mu=+200 \mathrm{GeV})$. The yellow and green bands represent the $\pm 1 \sigma$ and $\pm 2 \sigma$ variations around the expectation. The region excluded by the LEP experiments is also shown [33]. 


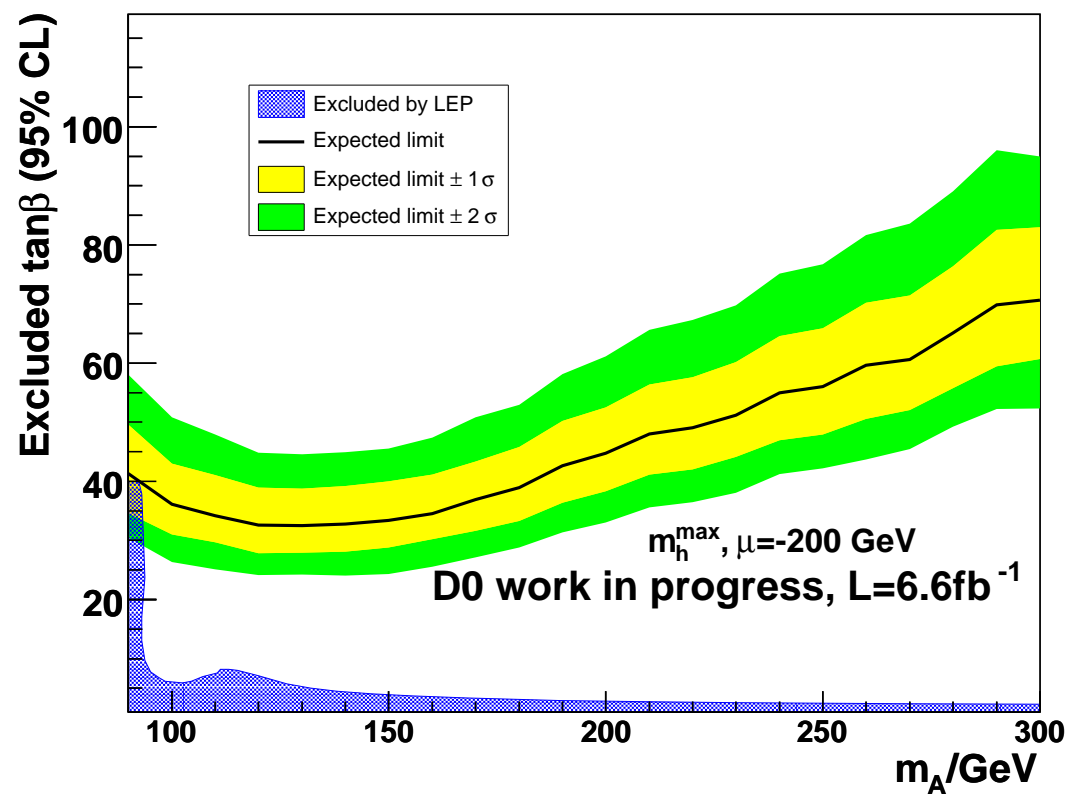

Figure 5.23: Expected confidence limits on $\tan \beta$ as a function of $m_{A}$, assuming the MSSM $m_{h}^{\max }$ $(\mu=-200 \mathrm{GeV})$ scenario. The yellow and green bands represent the $\pm 1 \sigma$ and $\pm 2 \sigma$ variations around the expectation. The region excluded by the LEP experiments is also shown [33]. 
Once again, these plots show increased sensitivity over the equivalents from the previous analysis version. These are also more sensitive than the latest CDF result in this channel [37].

\subsection{Conclusion}

Expected confidence limits have been set on the $\sigma \times$ BR of the process $\phi b \rightarrow b \bar{b} b$, and on the $m_{A}, \tan \beta$ plane for the MSSM for both a naive $\tan \beta$ enhancement and enhancements prescribed by accepted MSSM benchmark scenarios. The expected limits indicate that the sensitivity has been significantly increased by the use of an improved b-jet identification algorithm and an event-based analysis method: these limits show the best expected sensitivity for this analysis channel at the Tevatron. 


\section{Chapter 6}

\section{Conclusion and Outlook}

\subsection{Conclusion}

The Standard Model is currently the best available description of fundamental particle physics, with many experiments confirming its predictions. However, one key component of the SM, the Higgs boson, has yet to be observed. This thesis contains a description of a search for this process in the 3-4 b-jet final state (where the spectator b-jets aid signal identification). Searches in this channel are expected to be sensitive to MSSM (an extension of the Standard Model) Higgs bosons at high tan $\beta$.

The search was carried out on data from proton anti-proton collisions at the Tevatron particle accelerator collected by the $D \varnothing$ experiment. The Tevatron has been performing extremely well, providing a large amount of data to be analysed. In conjunction with this, DØ's well established b-jet identification methods, allows stringent confidence limits on the MSSM parameter space to be set in this channel.

The analysis reported in this thesis built on the previous $\mathrm{D} \varnothing \phi b \rightarrow b \bar{b} b$ analysis. As well as adding considerably more data, several significant improvements were introduced. A new trigger was used to collect extra data for the analysis (this trigger was commissioned in part by the author). In addition, the new MVA b-tagger, which is more efficient than the previously-used NN b-tagger, was used to select events: this analysis is among the first to use this b-tagger. The previous method, which analysed data using a jet-pair by jet-pair approach, was replaced with a method that took information from the whole event into account: information about three possible jet pairings in each event was used to train a multivariate analysis (MVA) algorithm to identify signal events from background. This MVA was also used to provide the discriminant used by the limit setting process: this replaced the use 
of the di-jet invariant mass distribution in the previous version of analysis. The expected limits on the $\sigma \times B R$ for the $\phi b \rightarrow b \bar{b} b$ process show that in addition to the gain in sensitivity due to analysing a larger dataset, the new method increased the sensitivity by roughly $30 \%$, with an additional $17 \%$ improvement coming from the MVA b-tagger. Overall, these changes significantly increased the expected sensitivity of the search.

\subsection{Outlook}

The 5.2 and $6.6 \mathrm{fb}^{-1}$ versions of the $\phi b \rightarrow b \bar{b} b$ analysis are in the final stages of approval by the $\mathrm{D} \varnothing$ collaboration. There are many potential improvements to the analysis that could be investigated. It is possible that new triggers (or combinations of existing triggers) could be used to provide more data to analyse. The b-tagging has a large bearing on the analysis so any improvements to b-tagging would lead to improvements in sensitivity. It is possible that the current b-tag requirement (three tight-tagged b-jets) is not optimised, and that some other combination (either using the discrete operating points or continuous variables) provides a better signal efficiency for similar background rate. The MVA b-tagger includes a tool for distinguishing b-jets from c-jets: this was not used in the current analysis, but given the suppression of $\phi \rightarrow c \bar{c}$ events in the MSSM, this tool could provide more powerful signal identification in this channel.

Additionally, the Random Forest can be further optimised: it is possible that more powerful discriminants than those used in this analysis are available. It is also possible that fine-tuning the parameters of the RF can bring added sensitivity, so the MVA technique is a possible area for a future study.

Sensitivity to physics beyond the Standard Model may be increased by combining results from this channel with other results: the results from the $\mathrm{D} \varnothing$ multijet analysis have been combined with the $\mathrm{D} \varnothing \tau^{+} \tau^{-}$channels [88], and combinations with CDF results are also possible (the $\mathrm{CDF}$ and $\mathrm{D} \varnothing \tau^{+} \tau^{-}$results have been combined in this way [89]). Also, the results from Standard Model Higgs boson searches can be reinterpreted to provide extra constraints on MSSM parameters [90]. In addition, the shapes of the systematic uncertainties (rather than their scales) have the dominant effect on the sensitivity, so the sensitivity is not limited by systematic uncertainties.

The Tevatron is performing well and is currently due to run to the end of 2011: by this point, $12 \mathrm{fb}^{-1}$ of integrated luminosity would be delivered to each detector. Conservative estimates (neglecting the improvements listed above) suggest that a $\tan \beta$ of 20 may be excluded with $10 \mathrm{fb}^{-1}$ of data per experiment. Beyond this, 
the Tevatron may continue running until the end of 2014, in which case a total integrated luminosity of $20 \mathrm{fb}^{-1}$ can be delivered to CDF and D $\varnothing$. The D $\varnothing$ detector continues to collect data with roughly $90 \%$ efficiency, allowing a large proportion of the delivered luminosity to be recorded for analysis. This growing dataset will allow the exclusion of a larger range of Higgs boson mass in the SM: a recent report [91] suggested that with $12 \mathrm{fb}^{-1}$ delivered to each experiment, the Tevatron experiments would show a combined sensitivity of $95 \%$ over the mass range favoured by fits to electroweak measurements. With a delivered integrated luminosity of $20 \mathrm{fb}^{-1}$ the sensitivity would increase to the $3 \sigma$ level. In all, the future of the CDF and D $\varnothing$ physics programmes is extremely promising. 


\section{References}

[1] The DØ Collaboration: Search for Neutral Higgs Bosons in Multi-b-Jet Events in $p \bar{p}$ Collisions at $\sqrt{2}=1.96$ TeV, Phys. Rev. Lett. 101, 221802 (2008)

[2] N. Osman, G. Davies \& J. Hays: Search for $H \rightarrow W W \rightarrow e \mu:$ A Jet Multiplicity Based Method, DØ Note 5884 (2009)

[3] N. Osman, G. Davies \& J. Hays: Investigating b-Tagging in the $Z H \rightarrow \nu \nu b \bar{b}$ Channel, DØ Note 6113 (2008)

[4] The Tevatron New Phenomenon Working Group: Combination of Tevatron Searches for the Standard Model Higgs Boson in the $W^{+} W^{-}$Decay Mode, Phys. Rev. Lett. 104, 061802 (2010)

[5] The DØ Collaboration: Search for Higgs Boson Production in Electron and Muon plus Missing Transverse Energy Final States with $6.7 \mathrm{fb}^{-1}$ of $\bar{p}$ Collisions at $\sqrt{s}=1.96 \mathrm{TeV}$, DØ Note 6082-CONF (2010)

[6] F. Halzen \& A. Martin: Quarks and Leptons, John Wiley and Sons (1984)

[7] S. Weinberg: A Model of Leptons, Phys. Rev. 127, 965 (1962)

[8] S. Glashow, J. Iliopoulos, L. Maiani: Weak Interactions with Lepton-Hadron Symmetry, Phys. Rev. D2 1285-1292, (1970)

[9] The Tevatron Electroweak Working Group: Updated Combination of CDF and DØ Results for the Mass of the W Boson, arXiv:0908.1374v1 [hep-ex] (2009)

[10] The LEP Electroweak Working Group and the SLD Electroweak and Heavy Flavour Groups: Precision Electroweak Measurements on the $Z$ Resonance, Phys. Rept. 427: 257 (2006)

[11] J. Goldstone, A. Salam \& S. Weinberg: Broken Symmetries, Phys. Rev. 127, 965 (1962) 
[12] P. Higgs: Broken Symmetries, Massless Particles and Gauge Fields, Phys. Lett. $12,132(1964)$

[13] P. Higgs: Broken Symmetries and the Masses of Gauge Bosons, Phys. Lett. 13, 508 (1964)

[14] P. Higgs: Spontaneous Symmetry Breakdown without Massless Bosons, Phys. Rev. 145, 508 (1966)

[15] K. Riesselmann: Limitations of a Standard Model Higgs Boson, hep$\mathrm{ph} / 9711456$ (1997)

[16] G. Bernardi, M. Carena, T. Junk: Higgs Bosons, Theory and Searches, (from the 2008 Review of Particle Physics, C. Amsler et al (Particle Data Group), Phys. Lett. B667

[17] The ALEPH experiment web page: http://aleph.web.cern.ch/aleph/

[18] The DELPHI experiment web page: http://delphiwww.cern.ch/

[19] The L3 experiment web page: http://13.web.cern.ch/13/

[20] The OPAL experiment web page: http://opal.web.cern.ch/Opal/

[21] The LEP Working Group for Higgs Boson Searches: Search for the Standard Model Higgs Boson at LEP, Phys. Rev. B 565: 61-75 (2003)

[22] The CDF Experiment web page: http://www-cdf.fnal.gov

[23] The DØ Experiment web page: http://www-d0.fnal.gov

[24] The Tevatron New Phenomenon Working Group: Combined CDF and DØ Upper Limits on Standard Model Higgs Boson Production with up to $6.7 \mathrm{fb}^{-1}$ of Data, CDF Note 10241, DØ Note 6096 (2010)

[25] The LEP Electroweak Working Group: A Combination of Preliminary, Electroweak Measurements and Constraints on the Standard Model, http: //lepewWg . web.cern.ch/LEPEWWG/plots/summer2010/

[26] D. I. Kazakov: Beyond the Standard Model, hep-ph/0012288 v2 (2000)

[27] S. P. Martin: A Supersymmetry Primer, hep-ph/9709356 v5 (2008)

[28] W. Yao et al.: (Particle Data Group): Supersymmetry, J. Phys. G 33. 1 (2006) 
[29] V. Barger \& C. Kao: Implications of New CMB Data for Neutralino Dark Matter, arXiv:hep-ph0106189 (2001)

[30] I. Aitchison: Supersymmetry and the MSSM: An Elementary Introdution, arXiv:hep-ph/0505105 v1 (2005)

[31] S. Dawson: Susy and Such, hep-ph/9612229 v2 (1997)

[32] B. Ananthanarayan, G. Lazarides, Q.Shafi: Top-Quark Mass Predictions from Supersymmetric Grand Unified Theories, Phys. Rev. D44, 16131615 (1991)

[33] The LEP Working Group for Higgs Boson Searches: Search for Neutral MSSM Higgs Bosons at LEP, Eur. Phys. J. C 47, 547 (2006)

[34] The CDF Collaboration: Search for Neutral MSSM Higgs Bosons Decaying to Tau Pairs with $1.8 \mathrm{fb}^{-1}$ of Data, CDF Note 9071 (2007)

[35] The DØ Collaboration: Search for Higgs Bosons Decaying to Tau Pairs in $p \bar{p}$ Collisions with the DØ Detector, Phys. Rev. Lett. 101, 071804 (2008)

[36] The DØ Collaboration: Search for the Associated Production of a $b$ Quark and a Neutral Supersymmetric Higgs Boson that Decays into $\tau$ Pairs, Phys. Rev. Lett. 104, 151801 (2010)

[37] The CDF Collaboration: Search for Higgs Bosons Produced in Association with b-Quarks, CDF Note 10105 (2010)

[38] The Fermi National Accelerator Laboratory (http://www.fnal.gov)

[39] S. Grünendahl: DØ Run Report, talk given at DØ Summer Workshop 2010

[40] Fermilab Beams Division (http://www-bdnew.fnal.gov)

[41] The DØ Collaboration: The Upgraded DØ Detector, Nucl. Instr. Meth. A(565):463-537 (2006)

[42] The DØ Collaboration: Beam Tests of the DØ Uranium Liquid Argon End Calorimeter, Nucl. Instr. Meth. A(324):53 (1993)

[43] A. Schwartzmann, C. Tully: Primary Vertex Reconstruction by Means of Adaptive Vertex Fitting, DØ Note 4918 (2005)

[44] E. Busato, B. Andrieu: Jet Algorithms in the DØ Run II Software: Description and User's Guide, DØ Note 4457 (2004) 
[45] DØ Single Top Observation, http://www-d0.fnal.gov/Run2Physics/top/singletop_observation/

[46] The DØ Collaboration: b-Jet Identification in the DØ Experiment, Nucl. Instr. Meth. A(620):490 (2010)

[47] L. Chabalina, R. Demina, A. Khanov \& F. Rizatdinova: http://www-d0.fnal.gov/phys_id/bid/d0_private/certification/p14/ CSIP/CSIPv2.html

[48] D. Bloch, B. Clément, D. Gelé, S. Greder \& I. Ripp-Baudot: Performance of the JLIP b-tagger in p14, DØ Note 4348 (2004)

[49] L. Feligioni, M. Narain, P. Schieferdecker \& A. Schwartzman: Update on bquark Jet Identification with Secondary Vertex Reconstruction using DØ Reco version p14, April 2004, DØ Note 4414 (2004)

[50] K. Hanagaki \& J. Kasper: Soft Lepton (muon) Tagging Method, http://www-d0.fnal.gov/phys_id/bid/d0_private/certification/p14/ muonjet/SLT v1.html

[51] T. Scanlon: b-Tagging and the Search for Neutral Supersymmetric Higgs Bosons at DØ, PhD Thesis: FERMILAB-THESIS-2006-43 (2006)

[52] T. Gadfort, A. Haas, D. Johnston, D. Lincoln, T. Scanlon \& S. Schlobohm: Performance of the DØ NN b-tagging Tool on p20 Data, DØ Note 5554 (2007)

[53] S. Greder \& I. Ripp-Baudot: B-tagging with combined Multivariate Techniques, DØ Note 5936 (2009)

[54] Level 3 Impact Parameter Jet b-tag Page:

http://www-d0.fnal.gov/computing/algorithms/level3/b-tagging/L3Btag.html

[55] T. Scanlon, S. Robinson: A Secondary Vertex b-Tagging Tool for the L3 Trigger, DØ Note 5292 (2006)

[56] V. Buescher, A. Duperrin, R. Jesik, A. Patwa, K. Stevenson, A. Turcot, B. Vachon \& M. Verzocchi: The Trigger Rate Tool Package, DØ Note Number 4640 (2004)

[57] R. Jesik, N. Osman: Including the L3 Secondary Vertex Tagger in the Trigger Rate Tool, DØ Note 5376 (2007) 
[58] G. Blazey DØ Performance and Planned Improvements, talk given to the Department of Energy High Energy Physics Advisory Panel, September 2005

[59] The DØ Operations Group: Daily Data Taking Efficiency, http://d0server1.fnal.gov/Projects/Operations/DORunII_DataTaking.htm

[60] D. Bauer, F.Couderc, G. Davies, A. Goussiou, J. Hays, P. Jonsson, P. Mal, T. Scanlon, B. Tuchming: Search for neutral Higgs bosons in $\Phi b \rightarrow b \bar{b} b$ using 5.2 $\mathrm{fb}^{-1}$ of RunII data, DØ Note 5942 (2010)

[61] M. Spira, Higgs Boson Production and Decay at the Tevatron, arXiv:hep$\mathrm{ph} / 9810289$ (1998)

[62] J. Campbell, R. Ellis, F. Maltoni \& S. Willenbrock: Higgs Boson Production in Association with a Single Bottom Quark, FERMILAB-Pub-02/062-T (2008)

[63] S. Greder, I. Ripp-Baudot: B-tagging with Combined Multivariate Techniques, DØ Note 5936

[64] Skim definitions:

http://www-d0.fnal.gov/Run2Physics/cs/skimming/p20_pass2_skims.html

[65] DØ data quality:

http://www-d0.fnal.gov/computing/data_quality/d0_private/forusers.html

[66] F.Couderc, B.Tuchming, G.Davies, P.Jonsson, S.Robinson, T.Scanlon: Search for neutral Higgs bosons at high tan $\beta$ in multijet events using 17 data, DØ Note 5341 (2007)

[67] Trigger List descriptions: http://www-d0.fnal.gov/trigger_meister/private/www/tl_desc/global.html

[68] G. Blazey et al., Run II Jet Physics arXiv:hep-ex/0005012 (2000).

[69] Jet Energy Scale web page: http://www-d0.fnal.gov/phys_id/jes/public_RunIIa

[70] N. Makovec \& J. Grivaz: Shifting, Smearing and Removing Simulated Jets, DØ Note 4914 (2005)

[71] M. L. Mangano, M. Moretti, F. Piccinini, R. Pittau \& A. D. Polosa: ALPGEN, a generator for hard multiparton processes in hadronic collisions, JHEP 0307, 001 (2003) 
[72] T. Sjöstrand, L. Lönnblad, S. Mrenna \& P. Skands: PYTHIA 6.3: Physics and Manual, arXiv:hep-ph/0308153 (2003)

[73] J. M. Campbell, R. K. Ellis: http://mcfm/.

[74] CTEQ Collaboration web page: http://www.phys.psu.edu/〜 cteq/

[75] S.Dutt, D. Lincoln \& G. Otero y Garzon: Taggability Studies in p20, D0 Note 5773, (2008)

[76] DØ b-Jet Identification web page: http://www-d0.fnal.gov/phys_id/bid/d0_private/bid.html

[77] S. Robinson: Neutral Supersymmetric Higgs Boson Searches at DØ, PhD Thesis: FERMILAB-THESIS-2009-40 (2008)

[78] R. Fletcher: Practical Methods of Optimization, second edition, Wiley (1987)

[79] TMultiLayerPerceptron in Root (http://root.cern.ch/root/html/TMultiLayerPerceptron.html)

[80] Y. Freund: Boosting a Weak Learning Algorithm by Majority, Information and Computing 121 pp. 256-285 (1995)

[81] Toolkit for Multivariate Data Analysis with ROOT (http://tmva.sourceforge.net)

[82] L.Breiman: Random Forests, Technical Report, University of California (2001)

[83] T. Andeen, B. Casey, K. DeVaughan, Y. Enari, E. Gallas, D. Krop, R. Partridge, H. Schellman, G. Snow, S. Yacoob \& H. Yoo: The DØ Experiment Integrated Luminosity for Tevatron RunIIa, FERMILAB-TM-2365, (2007)

[84] D. Bauer, G. Davies, J. Hays, P. Jonsson, T. Scanlon: A Trigger Parametrization for the $\Phi b \rightarrow b \bar{b} b$ analysis of RunIIb Data, DØ Note 5885 (2009)

[85] W. Fisher: Collie: A Confidence Level Limit Evaluator, DØ FERMILAB-TM2368-E (2007)

[86] : M. Frank, T. Hahn, S. Heinemeyer, W. Hollik, H. Rzehak \& G.Weiglein: The Higgs Boson Masses and Mixings of the Complex MSSM in the FeynmanDiagrammatic Approach, J. High Energy Phys. 02 (2007) 047. 
[87] M. Carena, S. Heinemeyer, C. E. M. Wagner \& G. Weiglein: Suggestions for Benchmark Scenarios for MSSM Higgs Boson Searches at Hadron Colliders, Eur. Phys. J. C 26: 601-607 (2003)

[88] The DØ Collaboration: Combined Upper Limits on MSSM Higgs-Boson Production with up to $2.6 \mathrm{fb}^{-1}$ of Data at DØ, DØ Note 5935 (2009)

[89] The Tevatron New Phenomena and Higgs Working Group: Combined CDF and DØ Upper Limits on MSSM Higgs Boson Production in tau-tau final states with up to 2. $6 \mathrm{fb}^{-1}$, FERMILAB-FN-0851-E (arXiv:1003.3363v3 [hep-ex]) (2010)

[90] P. Draper, T. Liu, C. Wagner: Prospects for MSSM Higgs Searches at the Tevatron, Phys. Rev. D 80, 035025 (2009)

[91] Fermilab Physics Advisory Committee Comments and Recommendations, August 2010

(http://www.fnal.gov/directorate/program_planning/phys_adv_com/PACdates.html) 